\title{
Ultra Wideband Wireless Communication Systems Under Narrowband Interference and Noisy Environments
}

\author{
by \\ Ehab Moustafa Fareed Shaheen, M.Sc. \\ A thesis submitted to the Faculty of Graduate Studies and Research \\ in partial fulfillment of the requirements for the degree of
}

Doctor of Philosophy in Electrical and Computer Engineering

Ottawa-Carleton Institute for Electrical and Computer Engineering (OCIECE)

Department of Systems and Computer Engineering

Carleton University

Ottawa, Ontario, Canada, K1S 5B6

August, 2010

(c) Copyright

Ehab Moustafa Fareed Shaheen, 2010 


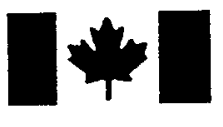

\section{Library and Archives \\ Canada}

Published Heritage

Branch

395 Wellington Street

Ottawa ON K1A ON4

Canada
Bibliotheque et

Archives Canada

Direction du

Patrimoine de l'édition

395 , rue Wellington

Ottawa ON K1A ON4

Canada
Your file Votre référence
ISBN: $978-0-494-70551-3$
Our file Notre référence
ISBN: $978-0-494-70551-3$
NOTICE:

The author has granted a nonexclusive license allowing Library and Archives Canada to reproduce, publish, archive, preserve, conserve, communicate to the public by telecommunication or on the Internet, loan, distribute and sell theses worldwide, for commercial or noncommercial purposes, in microform, paper, electronic and/or any other formats.

The author retains copyright ownership and moral rights in this thesis. Neither the thesis nor substantial extracts from it may be printed or otherwise reproduced without the author's permission.
AVIS:

L'auteur a accordé une licence non exclusive permettant à la Bibliothèque et Archives Canada de reproduire, publier, archiver, sauvegarder, conserver, transmettre au public par télécommunication ou par l'Internet, prêter, distribuer et vendre des thèses partout dans le monde, à des fins commerciales ou autres, sur support microforme, papier, électronique et/ou autres formats.

L'auteur conserve la propriété du droit d'auteur et des droits moraux qui protège cette thèse. Ni la thèse ni des extraits substantiels de celle-ci ne doivent être imprimés ou autrement reproduits sans son autorisation.
In compliance with the Canadian Privacy Act some supporting forms may have been removed from this thesis.

While these forms may be included in the document page count, their removal does not represent any loss of content from the thesis.
Conformément à la loi canadienne sur la protection de la vie privée, quelques formulaires secondaires ont été enlevés de cette thèse.

Bien que ces formulaires aient inclus dans la pagination, il n'y aura aucun contenu manquant.

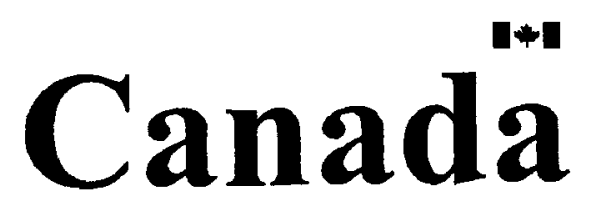




\section{Abstract}

Ultra wide-band is considered to be one of the most promising short range wireless communication technologies. This is due to its high data rate capability, very fine time-domain resolution allowing for location and tracking applications, resistance to dense multi-path environment and jamming. Despite its enviable benefits, the unlicensed usage of a very wide spectrum that overlaps with the spectra of narrowband technologies brings about interference related concerns. Therefore, significant researches have been carried out to quantify the interference issue in both sides.

This thesis investigates the impact of in-band narrowband interference signals on the performance of impulse radio ultra wide-band communication systems and proposes a mitigation technique to solve such problem.

The thesis presents bit error rate analyses for impulse radio, when subjected to narrowband interference over Log-normal fading as well as the IEEE802.15.3a fading channel models.

We modeled the narrowband interference signal as the standard IEEE802.11a wireless local area network, which is considered one of the main threats to ultra wide-band communication systems. It is shown that the presence of narrowband interference signal can severely deteriorate the performance of impulse radio ultra wideband communication systems.

To this end, an interference canceller scheme is proposed and its average bit error rate performance is investigated in various channel models. The studied channel models are the additive white Gaussian noise, Nakagami-m, Log-normal flat and frequency selective fading channels in addition to the ultra wide-band channel models developed by the IEEE802.15.3a task group.

A noticeable performance enhancement to UWB communication systems can be achieved with the use of the interference canceller scheme. Specifically, for an average probability of symbol error equals to 0.05 , the signal to noise ratio degradation is reduced to be less than $6 \mathrm{~dB}$ at bit error rate equals to $1 \times 10^{-4}$ in AWGN channel. 
While in a Log-normal flat fading channel, the degradation is less than $2 \mathrm{~dB}$ at bit error rate $=1 \times 10^{-3}$. In the CM1 IEEE802.15.3a UWB channel model, the degradation is less than $3 \mathrm{~dB}$ at bit error rate equals to $1 \times 10^{-3}$.

The effectiveness of all these analytical performance evaluations of the canceller scheme are supported by simulation. The performance of the proposed canceller scheme is also investigated in various scenarios of operation such as the presence of symbol timing error and the presence of multiple narrowband interference signals. The impact of multiple access interference on the performance of such canceller is also presented.

Finally, we gave a comparison with the performance obtained by using a "Notch filter" based case, characterized by different quality factors. The comparison is done in two scenarios: perfectly tuned and de-tuned notch filter. 
To the memory of my father

Moustafa Fareed Shaheen, (1940-2007)

for his unconditional inspiring, encouraging and love.

May God bless his soul. 


\section{Acknowledgments}

Above all, I wholeheartedly thank my mighty $\boldsymbol{G o d}$ for giving me the vision and the power to believe in my passion and pursue my dreams. I could never have done this without the faith I have in you.

My greatest debt is to my thesis supervisor Prof. Mohamed El-Tanany for his guidance, suggestion, encouragement and support. I am so grateful to him for leading me into this cxciting research area. I will continue to be influenced by his clarity in thinking and professional integrity.

I also wish to thank all the members of my proposal examining board for giving me constructive suggestions and advices.

In addition, I would like to thank the secretarial staff in the Department of Systems and Computer Engineering for all their help.

At last, I would like to thank my wife and my son for their love, support, patience, cooperation and understanding which make the last few years so memorable. 


\section{Table of Contents}

Abstract

Acknowledgments $\quad$ ii

Table of Contents $\quad$ iii

List of Tables $\quad$ vi

List of Figures $\quad$ vii

Nomenclature xiv

1 Introduction 1

1.1 Research Motivation . . . . . . . . . . . . . . . . . . 1

1.2 Thesis Contributions . . . . . . . . . . . . . . . 2

1.3 General Assumptions and Shortcomings . . . . . . . . . . . . 3

1.4 Thesis Organization . . . . . . . . . . . . . . . . 4

2 Literature Review 6

2.1 Introduction . . . . . . . . . . . . . . . . . 6

2.2 UWB Communication Background . . . . . . . . . . . . 6

2.3 Data Modulation and Multiple Access Schemes . . . . . . . . . . . 9

2.3.1 Data Modulation Methods . . . . . . . . . . . . . . . . . 9

2.3.2 Multiple Access Methods . . . . . . . . . . . . . . . . . . . . . 14

2.4 UWB Pulse Shape . . . . . . . . . . . . . . . . . . . . . . . 19

2.5 UWB Propagation Channel . . . . . . . . . . . . . . . . . 22

2.6 Global Regulations on UWB . . . . . . . . . . . . . . . . . 25

2.6.1 UWB regulations in the United States . . . . . . . . . 25

2.6.2 UWB regulations in the European Union . . . . . . . . . . 25 
2.6.3 UWB regulations in Japan . . . . . . . . . . . . . . . 28

2.7 Types of Interference . . . . . . . . . . . . . . . . . . . . . . . 29

2.7 .1 Multiple Access Interference . . . . . . . . . . . . . . . . . . . . . . . . . . . . . . . . 39

2.7.2 Inter Symbol Interference . . . . . . . . . . . . . 30

2.7 .3 Narrowband Interference . . . . . . . . . . . . . . . . 30

2.8 Survey of NBI Suppression Techniques . . . . . . . . . . . . . . . 33

2.8.1 NBI Avoidance Techniques . . . . . . . . . . . . 33

2.8.2 Physical NBI Avoidance Techniques . . . . . . . . . . . . . . . . . . . . . . . . . . . 36

2.8.3 NBI Cancellation Techniques . . . . . . . . . 36

3 The Interference Canceller Scheme (ICS) 42

3.1 Introduction . . . . . . . . . . . . . . . . . 42

3.2 The Impact of UWB Interference on Narrowband Receiver . . . . . . 44

3.3 Performance of the ICS in AWGN Channel . . . . . . . . . . . . 45

3.4 IR-UWB Performance in the Presence of NBI in AWGN Channel . . 50

3.5 Performance of the ICS in Nakagami-m Flat Fading Channels . . . . 55

3.6 Summary of the ICS BER Performance . . . . . . . . . . . 58

4 Simulation of the ICS $\quad \mathbf{6 4}$

4.1 Simulation of the IR-UWB Communication System . . . . . . . . . 64

4.1.1 TH-PPM Transmission Scheme . . . . . . . . . . . . 64

4.1.2 DS-BPSK Transmission Scheme . . . . . . . . . . . . . 66

4.1 .3 IR-UWB Reception Scheme . . . . . . . . . . . . . . . 67

4.1.4 Simulation of the IEEE802.15.3a UWB Propagation Channel . 71

4.2 Simulation of the Standard IEEE802.11a WLAN System . . . . . . . 74

4.2 .1 Transmitter ..................... 74

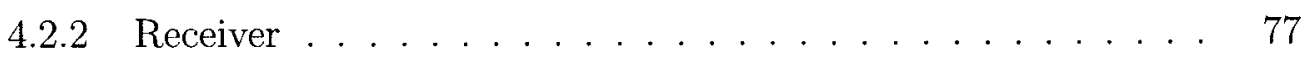

4.2 .3 Simulation of the NBI Channel . . . . . . . . . . . 78

4.3 Simulation of the ICS . . . . . . . . . . . . . 80

5 BER Analysis in Various UWB Channel Models 83

5.1 BER Analysis of a UWB System in the Presence of NBI in Log-normal Multi-path Fading Channels . . . . . . . . . . . . . . 83

5.1 .1 Performance Evaluation . . . . . . . . . . . . 85

5.1 .2 Analytical Validation . . . . . . . . . . . . . . . . . . 91

5.1 .3 Numerical Results . . . . . . . . . . . . . . . . . 99 
5.2 BER Analysis of a UWB system in the Presence of NBI in Log-normal Flat Fading Channels . . . . . . . . . . . . . . . . . . . . . . 99

5.2 .1 Performance Evaluation . . . . . . . . . . . . . . . 104

5.2 .2 Numerical Results . . . . . . . . . . . . . . . . . 107

5.3 BER Analysis of a UWB system in the Presence of NBI in the IEEE802.15.3a UWB Channel Models . . . . . . . . . . . . . . . . 112

5.3.1 Performance Evaluation . . . . . . . . . . . . . . . . 114

5.3 .2 Numerical Results . . . . . . . . . . . . . . . . . . . 118

5.4 Performance of the ICS in Various UWB Channel Models . . . . . . 124

5.4.1 ICS Performance in a Log-normal Flat Fading Channel . . . 124

5.4.2 ICS Performance in the IEEE802.15.3a UWB Channel Models 126

5.5 Comparison between the ICS Analytical and Simulation Results . . 126

6 ICS Performance in Various Scenarios 132

6.1 ICS Performance in the Presence of Multiple NBI Signals . . . . . . 132

6.2 ICS Performance in the Presence of Symbol Timing Error . . . . . . 136

6.3 ICS Performance in the Presence of MAI . . . . . . . . . . . 139

6.3.1 AWGN Channel Environment . . . . . . . . . . . . . 140

6.3.2 Nakagami-m and Log-normal Flat Fading Channels . . . . . 152

6.3.3 In the IEEE802.15.3a UWB Channel Models . . . . . . . . . . 164

6.4 Performance Comparison with a Notch Filter Based Case . . . . . . 168

6.5 IEEE 802.11n Interference on IR-UWB Systems $\ldots \ldots \ldots \ldots \ldots$

6.5.1 Overview of the IEEE802.11n Standard . . . . . . . . . . 172

6.6 IEEE802.11n Interference Analysis . . . . . . . . . . . . . 177

7 Conclusions and Suggestions for Future Work 179

7.1 Conclusion and Summary . . . . . . . . . . . . . . . . . . 179

7.2 Suggestions for Future Research . . . . . . . . . . . . . . . . 182

List of References $\quad 184$ 


\section{List of Tables}

2.1 A comparison of indoor UWB data rate with other wireless standards. 8

2.2 The $-10 \mathrm{~dB}$ bandwidth relation with the Gaussian pulse order of differentiation and the Gaussian pulse standard deviation [1]. . . . . . 22

2.3 Parameter Settings for the IEEE UWB Channel Model [2]. . . . . . . 25

2.4 Summary of the NBI Mitigation Techniques . . . . . . . . . . . . 41

4.1 The used UWB simulated parameters value. . . . . . . . . . . . . 68

4.2 The used IEEE802.11a simulated parameters value. . . . . . . . . 78

6.1 Specifications of the IEEE802.11a/n Systems. . . . . . . . . . . . 175

6.2 Summary of IEEE802.11n $40 \mathrm{MHz}$ Channel Allocation in the $5 \mathrm{GHz}$ Frequency Band $[3] \ldots \ldots \ldots . \ldots \ldots 175$ 


\section{List of Figures}

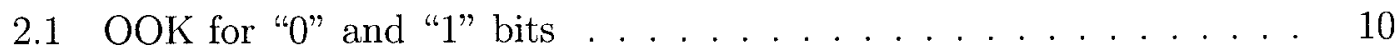

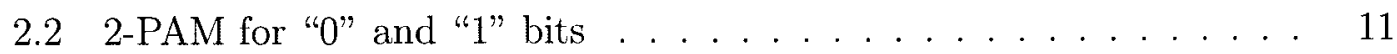

2.3 Bi-phase modulation for "0" and " 1 " bits . . . . . . . . . 12

2.4 PPM modulation scheme for "0" and "1" bits . . . . . . . . . . 14

2.5 Sample of UWB DS-BPSK Waveform for two bits ( 1,0 respectively)

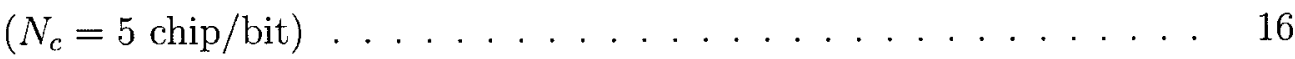

2.6 Sample of UWB TH-PPM Waveform for two bits ( 1,0 respectively) $\left(N_{s}=5\right.$ pulse $/$ bit,$\left.N_{h}=3\right) \ldots \ldots \ldots \ldots \ldots \ldots$

2.7 The impact of varying the pulse shaping factor on the peak energy frequency value for the first 15 derivatives of the UWB Gaussian pulse. 20

2.8 The impact of varying the pulse shaping factor on the $-10 \mathrm{~dB}$ bandwidth value for the first 15 derivatives of the UWB Gaussian pulse. . 21

2.9 FCC UWB Indoor and Outdoor emission Masks [4] . . . . . . . . 26

2.10 European UWB emission Mask [4]. . . . . . . . . . . . . . . 27

2.11 Japan UWB Indoor emission Mask [4]. . . . . . . . . . . . . 28

2.12 Narrowband interference threats over the FCC UWB spectral mask for communication systems $[5] . \ldots \ldots \ldots . \ldots . \ldots . \ldots 32$

2.13 (a):Dual-band approach as proposed by the Xtreme SpectrumMotorola [6], (b): The multi-band OFDM [7] . . . . . . . . 37

2.14 Configuration of the single and dual band notches UWB antenna [8] . 38

3.1 A general block diagram of the ICS. . . . . . . . . . . . . 43

3.2 Analytic evaluation of the BER performance of a QPSK-OFDM narrowband system in AWGN channel in the presence of UWB interference signal and $B_{u w b} / B_{o f d m}=125 \ldots \ldots \ldots \ldots \ldots \ldots$

3.3 BER of QPSK-OFDM narrowband system in AWGN channel in presence of UWB interference signal, $E_{b} / N_{o}=10 \mathrm{~dB} \ldots \ldots \ldots 47$ 
3.4 NBI receiver probability of symbol error with " $\mathrm{e}=1$ " symbol errors over a block $\mathrm{N}=52$ symbols at different $\mathrm{SIR}=0$, and $-20 \mathrm{~dB} . \ldots 49$

3.5 Probability of e symbol errors in a block of length $\mathrm{N}=52$ at $\mathrm{SIR}=0 \mathrm{~dB}$ and $B_{u w b} / B_{o f d m}=125 \ldots \ldots \ldots \ldots$. . . . . . . . . . 51

3.6 BER performance of the TH-PPM system in the presence of $N_{i}=52$ tone interferers. . . . . . . . . . . . . . . . . . . . 54

3.7 BER performance of the ICS for one symbol error case (one tone interferer . . . . . . . . . . . . . . . . . 56

3.8 BER performance of the ICS for multiple symbol errors case. . . . . . 57

3.9 BER performance of a UWB system in presence of IEEE802.11a NBI signal for different data rates and different values of the Nakagami fading parameter $\mathrm{m}, \mathrm{SIR}=-10 \mathrm{~dB}$. . . . . . . . . . . 59

3.10 UWB BER performance comparison with/without the ICS in a Nakagami flat fading channel, $\mathrm{m}=2$, av. $P_{s e}=0.1$ and $\mathrm{SIR}=-15 \mathrm{~dB} .$.

3.11 UWB BER performance comparison with/without the ICS in a Nakagami flat fading channel, $\mathrm{m}=8$, av. $P_{\text {se }}=0.1$ and $\mathrm{SIR}=-15 \mathrm{~dB} \ldots .61$

3.12 ICS BER performance in AWGN channel, SIR $=-20 \mathrm{~dB} . \ldots . . . .62$

3.13 ICS BER performance in a Nakagami-m flat fading channel, SIR $=-20$ dB . . . . . . . . . . . . . . . . . . 63

4.1 The Block diagram of the TH-PPM-UWB Transmission System [9]. . 65

4.2 The Block diagram of the DS-BPSK-UWB Transmission System [9]. . 66

4.3 A single correlator UWB receiver scheme. . . . . . . . . . . . . 67

4.4 Simulation and Analytical evaluation of the BER performance of the TH-PPM system. . . . . . . . . . . . . . . . . . . . .

4.5 Simulation and Analytical evaluation of the BER performance of the TH-PPM system, showing the confidence interval of the simulation results. . . . . . . . . . . . . . . . . .

4.6 A realization of the discrete time impulse response for the CM1 and the CM2 channel models.

4.7 A realization of the discrete time impulse response for the CM3 and the CM4 channel models. . . . . . . . . . . . . . . . 73

4.8 Simulation blocks of the NBI IEEE802.11a transmission scheme [10]. 75

4.9 Data Mapping scheme. . . . . . . . . . . . . . . . 76

4.10 Frame format of the simulated WLAN IEEE802.11a system. . . . . . 76

4.11 Reception Scheme of the WLAN IEEE802.11a narrow band system [10] 77 
4.12 Comparison between the simulated and analytical performances of the IEEE802.11a system in AWGN channel, showing the confidence interval for the simulated results. . . . . . . . . . . . . . . .

4.13 Realization scheme to simulate the Rayleigh distributed NBI fading channel [11]. . . . . . . . . . . . . . . . . . . 81

4.14 Simulation block diagram of the Canceller scheme. . . . . . . . . . 82

5.1 Schematic model for a UWB receiver system with a single tone interferer, additive noise and the associate fading channels. . . . . . . .

5.2 PDF relation between Nakagami-m and Log-normal distributions for Low values of $\mathrm{m} . \ldots \ldots \ldots \ldots$

5.3 PDF relation between Nakagami-m and Log-normal distributions for high values of $\mathrm{m} . \ldots \ldots \ldots \ldots$

5.4 TH-PPM BER performance in a Nakagami-m flat fading channel for different values of $\mathrm{m}$ and $\mathrm{SIR}=-10 \mathrm{~dB} \ldots \ldots \ldots$

5.5 TH-PPM BER performance in the presence of a single tone interferer $(\mathrm{SIR}=-10 \mathrm{~dB})$, for $\left(\mathrm{m}=4, \eta_{0}=0.07, \eta_{1}=1.3569, \eta_{2}=0.9146\right), \quad(\mathrm{m}=8$, $\left.\eta_{o}=0.04, \eta_{1}=1.2927, \eta_{2}=0.9289\right)$, and $\left(\mathrm{m}=10, \eta_{o}=0.034, \eta_{1}=1.2914\right.$, $\left.\eta_{2}=0.9281\right) \ldots \ldots \ldots \ldots \ldots \ldots$

5.6 TH-PPM BER performance in the presence of a single tone interferer $(\mathrm{SIR}=-10 \mathrm{~dB})$, for $\left(\mathrm{m}=20, \eta_{o}=0.0205, \eta_{1}=1.2289, \eta_{2}=0.9427\right)$, and $\left(\mathrm{m}=75, \eta_{o}=0.0102, \eta_{1}=1.1844, \eta_{2}=0.9526\right) \ldots \ldots \ldots$

5.7 TH-PPM performance in a flat fading channel, with a single tone interferer under Log-normal and Nakagami-m distributions for $m=75$. .

5.8 TH-PPM performance in the presence of a single tone interferer in a

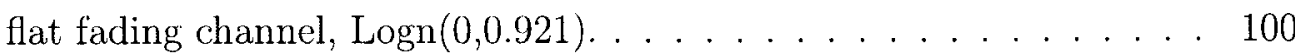

5.9 TH-PPM performance in the presence of a single tone interferer in a frequency selective fading channel, $\mathrm{L}=8$ paths, $\operatorname{Logn}(0.25,0.5296)$ using relations between equation (5.26) and equation (5.28) . . . . .

5.10 The effect of changing the $\mathrm{dB}$ spread values on the performance of the TH-PPM system in presence of a single tone interferer in a frequency selective fading channel, $\mathrm{L}=8$ paths and $\mu_{r}=0.25 \ldots \ldots . . .102$

5.11 BER performance of the TH-PPM system in a Log-normal flat fading channel in the presence of a single tone interferer compared with the analytic result of equation (5.51), dB-spread $=3 \mathrm{~dB}$. Analytic results are also compared with simulation. 
5.12 BER performance of the TH-PPM system in the presence of a single tone interferer at different dB-spread values [2-5 dB]. Analytic results are also compared with simulation. . . . . . . . . . . . . . . . 109

5.13 BER performance of the DS-BPSK system in a Log-normal flat fading channel in the presence of a single tone interferer, dB-spread $=3 \mathrm{~dB}$. Analytic results are compared with simulation and with the analytic results obtained from equation (5.51) . . . . . . . . . . . . . . . . 110

5.14 Single tone versus two tone interferers impact on the TH-PPM system. dB-spread $=3 \mathrm{~dB} . \ldots \ldots \ldots$

$5.15 \mathrm{BER}$ performance of the MRC-Rake receiver in presence of IEEE802.11a in the CM1 channel model, $\mathrm{L}=2, \mathrm{~K}=20$ for different SIRs. . . . . . . . . . . . . . . . . . . . . 119

5.16 BER performance of the MRC-Rake receiver in presence of IEEE802.11a in the CM4 channel model, $\mathrm{L}=2, \mathrm{~K}=20$ for different SIRs. . . . . . . . . . . . . . . . . . . . . .

5.17 Simulation versus analytic evaluation of the BER performance of the MRC-Rake receiver in the presence of IEEE802.11a in the CM1 and the CM4 channel models. . . . . . . . . . . . . . . . . . .

5.18 A comparison between the derived analytic performance presented in equation (5.84) (in presence of a single WLAN NBI signal) and the simulation experiment presented in [12] in the CM1 channel model in presence of " 8 " interfering WLAN channels and for 10-fingers MRC Rake receiver (the total interference power is the same in both cases).

5.19 Impact of changing the number of Rake receiver fingers on the performance of the UWB system in the CM1 and the CM4 channel models, $\mathrm{SIR}=-30 \mathrm{~dB}, \mathrm{~L}=2 \ldots \ldots \ldots \ldots \ldots$

5.20 ICS BER performance in the presence of the IEEE802.11a NBI in a Log-normal flat fading channel, SIR $=-10 \mathrm{~dB} \ldots \ldots \ldots . \ldots 125$

5.21 ICS BER performance in the CM1 channel model, SIR $=-20 \mathrm{~dB} . \quad . \quad 127$

5.22 ICS BER performance in the CM4 channel model, SIR $=-20 \mathrm{~dB} . \quad$. 128

5.23 Simulation and analytical performance comparison of the ICS in

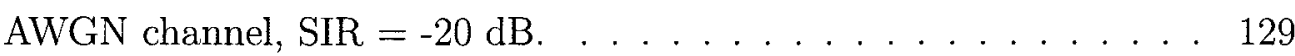

5.24 Simulation and analytical performance comparison of the ICS in a Nakagami-m flat fading channel, $\mathrm{m}=2$ and $\mathrm{SIR}=-20 \mathrm{~dB} \ldots \ldots . .130$ 
5.25 Simulation and analytical performance comparison of the ICS in a Lognormal flat fading channel, $\mathrm{dB}$-spread $=3 \mathrm{~dB}$ and SIR $=-10 \mathrm{~dB} . \quad \ldots 131$

6.1 The ICS in the presence of two NBI signals. . . . . . . . . . . 133

6.2 BER performance of the ICS in the presence of one and two NBI WLAN signals in AWGN channel, SIR $=-15 \mathrm{~dB} . \ldots \ldots \ldots . . .134$

6.3 BER performance of the ICS in the presence of two NBI WLANs in a Log-normal flat fading channel, SIR $=-10 \mathrm{~dB} \ldots \ldots \ldots \ldots . \ldots 135$

6.4 ICS BER performance in the presence of one and two NBI WLANs in the CM1 channel model. . . . . . . . . . . . . . . . . . . . 136

6.5 ICS BER performance in the presence of one and two NBI WLANs in the CM4 channel model. . . . . . . . . . . . . . . . . . . 137

6.6 BER performance of the canceller scheme in the presence of symbol timing error in AWGN channel. . . . . . . . . . . . . . . 138

6.7 Timing error impact in a Log-normal flat fading channel, $\mathrm{dB}$-spread $=$ $2 \mathrm{~dB}$ and $\mathrm{SIR}=-15 \mathrm{~dB} \ldots \ldots \ldots \ldots \ldots \ldots$

6.8 Symbol Timing error in the CM1 channel model. . . . . . . . . . . 140

6.9 Symbol Timing error in the CM4 channel model. . . . . . . . . . 141

6.10 BER performance of a TH-PPM system in the presence of MAI . . . 143

6.11 BER performance of a TH-PPM system in the presence of MAI and NBI signals . . . . . . . . . . . . . . . . . . . . 145

6.12 BER performance of the ICS in the presence of MAI and NBI with an

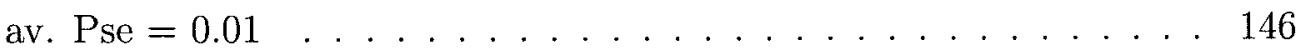

6.13 BER performance of the ICS in the presence of MAI and NBI with an av. Pse $=0.1 \ldots \ldots \ldots \ldots \ldots \ldots \ldots \ldots \ldots$

6.14 Comparison between the BER performances of a TH-PPM system in the presence of PPC and IPC MAI. . . . . . . . . . . . . . 148

6.15 Comparison between the BER performances of a TH-PPM UWB system in the presence of NBI along with PPC and IPC MAI for 20 users. 149

6.16 Comparison between the ICS BER performance in the presence of NBI, PPC and IPC MAI for 20 users, SIR $=-20 \mathrm{~dB}$ and with av. Pse $=0.01 .150$

6.17 Comparison between the ICS BER performance in the presence of NBI, PPC and IPC MAI for 20 users, SIR $=-20 \mathrm{~dB}$ and with av. Pse $=0.1 .151$

6.18 BER performance of a TH-PPM UWB system in the presence of PPC MAI and NBI in a Nakagami-m flat fading channel. . . . . . . . . . 153 
6.19 ICS BER performance in the presence of PPC MAI and NBI in a Nakagami-m flat fading channel, $\mathrm{m}=2, \mathrm{SIR}=-20 \mathrm{~dB}$ and av. $P_{s e}=$

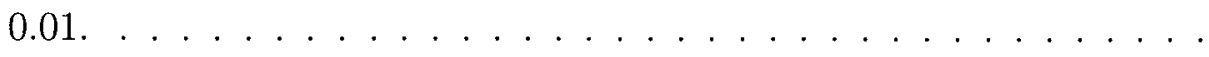

6.20 ICS BER performance in the presence of PPC MAI and NBI in a Nakagami-m flat fading channel, $\mathrm{m}=2, \mathrm{SIR}=-20 \mathrm{~dB}$ and av. $P_{\text {se }}=$ $0.1 \ldots \ldots \ldots \ldots \ldots \ldots \ldots$

6.21 BER performance of a TH-PPM system in the presence of PPC MAI and NBI in a Log-normal flat fading channel. . . . . . . . . . . . . 156

6.22 ICS BER performance in the presence of PPC MAI and NBI in a Lognormal flat fading channel, av. $P_{s e}=0.01, \mathrm{~dB}$-spread $=2 \mathrm{~dB}$ and SIR $=-20 \mathrm{~dB}$

6.23 ICS BER performance in the presence of PPC MAI and NBI in a Lognormal flat fading channel, av. $P_{s e}=0.1, \mathrm{~dB}$-spread $=2 \mathrm{~dB}$ and SIR $=-20 \mathrm{~dB}$.

6.24 BER performance comparison of a TH-PPM system in PPC and IPC MAI in the presence of NBI in a Nakagami-m flat fading channel, $N_{u}$ $=20$ users and $\mathrm{m}=2 \ldots \ldots \ldots \ldots \ldots$

6.25 ICS BER performance in PPC and IPC MAI in a Nakagami-m flat fading channel, av. Pse $=0.01, N_{u}=20$ users and $\mathrm{m}=2 . \ldots 160$

6.26 ICS BER performance in PPC and IPC MAI in a Nakagami-m flat fading channel, av. Pse $=0.1, N_{u}=20$ users and $\mathrm{m}=2 \ldots \ldots 1$

6.27 ICS BER performance in the presence of PPC and IPC MAI in a Lognormal flat fading channel, av. $P_{s e}=0.01$ and $N_{u}=20$ users. . . .

6.28 ICS BER performance in the presence of PPC and IPC MAI in a Lognormal flat fading channel, av. $P_{s e}=0.1$ and $N_{u}=20$ users. . . . . 163

6.29 BER performance of the DS-BPSK system in the presence of NBI and PPC MAI in the CM1 channel model. . . . . . . . . . . . . . . . . . 165

6.30 BER performance of the DS-BPSK-UWB in the presence of NBI and PPC MAI in the CM4 channel model. . . . . . . . . . . . . . . . . . 166

6.31 BER performance of the ICS in the presence of NBI and PPC MAI in the CM1 channel model and SIR $=-20 \mathrm{~dB} . . . . . . . . .$.

6.32 BER performance of the ICS in the presence of NBI and PPC MAI in the CM4 channel model and SIR $=-20 \mathrm{~dB} \ldots \ldots \ldots 168$

6.33 ICS performance in the presence of PPC and IPC MAI in the CM1 channel model and SIR $=-20 \mathrm{~dB}$ 
6.34 ICS performance in the presence of PPC and IPC MAI in the CM4 channel model and SIR $=-20 \mathrm{~dB} \ldots \ldots \ldots 170$

6.35 Performance comparison with a notch filter in the CM1 channel model and $\mathrm{SIR}=-15 \mathrm{~dB} . \ldots \ldots \ldots \ldots 171$

6.36 Performance comparison with a notch filter in the CM4 channel model and SIR $=-15 \mathrm{~dB} . \ldots \ldots \ldots 172$

6.37 Performance comparison with a de-tuned notch filter in the CM1 channel model, $\mathrm{Q}=100$ and $\mathrm{SIR}=-15 \mathrm{~dB} \ldots \ldots . \ldots 173$

6.38 Performance comparison with a de-tuned notch filter in the CM4 channel model, $\mathrm{Q}=100$ and $\mathrm{SIR}=-15 \mathrm{~dB} \ldots \ldots . \ldots 174$

6.39 Data mapping scheme for the IEEE802.11n WLAN. . . . . . . . . . 176

6.40 IEEE802.11a versus IEEE802.11n impact on a TH-PPM UWB system in AWGN channel. . . . . . . . . . . . . . . . 178 


\section{Nomenclature}

\section{List of Acronyms}

Acronyms

AWGN

ARake

av. Pse

BER

CDMA

DS

DS-BPSK

FCC

FDD

FDMA

FEC

GI

GPS

ICS

i.i.d.

IPC

IR

\section{Definition}

Additive white Gaussian noise

All-Rake

Average probability of OFDM symbol error

Bit error rate

Code division multiple access

Direct sequence

Direct sequence binary phase shift keying

Federal Communications Commission

Frequency division duplex

Frequency division multiple access

Forward error correction

Guard interval

Global positioning systems

Interference canceller scheme

Independent and identically distributed

Imperfect power control

Impulse radio 


\section{Acronyms}

ISI

LMS

LOS

MAC

MAI

MF

MIMO

MMSE

MHPF

ML

$\mathrm{MRC}$

NBI

NLOS

OFDM

OOK

PAM

PDF

PPC

PPM

PSD

PSM

PSWF

\section{Definition}

Inter symbol interference

Least mean squares

Line-of-sight

Medium access control

Multiple access interference

Matched filter

Multiple-input multiple-output

Minimum mean square error

Modified Hermite polynomial functions

Maximum likelihood

Maximal ratio combiner

Narrow band interference

Non line-of-sight

Orthogonal frequency division multiplexing

On-off keying

Pulse amplitude modulation

Probability density function

Perfect power control

Pulse position modulation

Power spectral density

Pulse shape modulation

Prolate spheroidal wave functions 


\section{Acronyms}

QoS

QPSK

$\mathrm{RF}$

R.V.

SIR

$S I R_{o}$

SGA

SNIR

SNR

SRake

$\mathrm{S}-\mathrm{V}$

TDD

TDMA

$\mathrm{TH}$

TH-PPM

UMTS

UWB

$\mathrm{Wi}-\mathrm{Fi}$

WLAN

\section{Definition}

Quality of service

Quadrature phase shift keying

Radio frequency

Random variable

Signal to interference ratio as seen

by a UWB receiver

Signal to interference ratio as seen

by an OFDM narrowband receiver

Standard Gaussian approximation

Signal to noise plus interference ratio

Signal to noise ratio

Selective-Rake

Saleh-Valenzuela channel model

Time-division duplex

Time division multiple access

Time hopping

Time hopping pulse position modulation

Universal mobile telephone service

Ultra wideband

Wireless Fidelity

Wireless local area network 


\section{List of Symbols}

$\begin{array}{ll}\text { Symbol } & \text { Explanation } \\ B_{o f d m} & \text { The OFDM bandwidth } \\ B_{U W B} & \text { The UWB bandwidth } \\ c_{j}^{k} & \text { Time-hopping sequence for the } k^{\text {th }} \text { user } \\ C_{I}(t), C_{Q}(t) & \text { The in-phase and quadrature components respectively } \\ E_{b} & \text { Energy per Bit } \\ E_{p} & \text { The transmitted energy per pulse } \\ E(\mathrm{x}) & \text { Expectation of x } \\ E_{R X}^{(1)}, E_{R X}^{(n)} & \text { The received energies for the desired user and } \\ f_{c} & \text { the } n^{t h} \text { interfering user respectively } \\ f_{H} & \text { Center frequency } \\ & \text { The upper 10dB point of the UWB signal power } \\ f_{L} & \text { spectrum } \\ I_{n} / C & \text { The lower 10dB point of the UWB signal power } \\ J_{o}(\omega) & \text { spectrum } \\ k_{m} & \text { PDF of x } \\ f_{x}(x) & \text { The NBI channel impulse response } \\ h_{i}(t) & \text { The UWB channel impulse response } \\ h_{s}(t) & \text { Thterference total power } \\ & \text { The average SIR ratios corresponding to the } n^{t h} \text { interferer }\end{array}$




\section{Symbol}

K

L

$\mathrm{m}$

$m(t)$

$|M(f)|$

$\mathrm{n}(\mathrm{t})$

$n_{1}, n_{2}$

ns

$N_{c a}$

$N_{c}$

$N_{m}$

$N_{o}$

$N_{p}$

$N_{s}$

$N_{u}$

$p(t)$

$P(f)$

Q(.)

Q

$r(t)$

$R_{b}$

\section{Explanation}

The number of rays in the multi-path fading model

The number of clusters in the multi-path fading model

The Nakagami-m fading parameter

The UWB correlation mask

The transfer function of the matched filter

Noise signal

The fading on each cluster and ray respectively

The number of symbols transmitted per packet in one simulated loop

The number of NBI sub-carriers

Number of pulses per data bit

The maximum number of bins considered within a single

observation interval

The spectral noise density

The period of the pseudo random code

Number of frames per information bit

The total number of transmitting users

UWB pulse wave form

The fourier transform of the UWB pulse

Q-function, the area under the tail of the Gaussian PDF

Filter quality factor

Received signal

The signal data rate 


\section{Symbol}

$R_{o}(\tau)$

$S_{o f d m}$

$S_{u w b}$

t

$T_{b}$

$T_{c}$

$T_{f}$

$T_{m}$

$w_{i}, b_{i}$

$W_{o}$

$\mathrm{X}$

Z

$\alpha_{k, \ell}$

$T_{\ell}$

$\tau_{k, \ell}$

${ }_{2} F_{1}(., . ; . ;$.

$\aleph_{k, \ell}$

$\left|\beta_{0,0}\right|^{2}$

$\beta_{k, \ell}$

$\delta$

\section{Explanation}

The autocorrelation function of the UWB signal

The OFDM signal power

The UWB signal power

Time (variable)

Bit duration

Direct sequence chip duration

UWB frame duration

The UWB pulse duration

The weights and the associated roots of the Hermite

polynomial respectively

The Lambert $\mathrm{W}$ function

The Log-normal shadowing

Decision statistic

Multi-path gain coefficient for cluster $\ell$ and ray $\mathrm{k}$

The delay of the $\ell^{\text {th }}$ cluster

The delay of the $k^{\text {th }}$ ray relative to the arrival time

of the $\ell^{\text {th }}$ cluster

The Gauss hypergeometric function

The normalized power dispersion profile

The mean energy of the first path of the first cluster

The fading associated with the $k^{t h}$ ray of the

$\ell^{\text {th }}$ cluster

The time hopping modulation index 


\section{Symbol}

$\delta($.

$\Delta f$

$\Delta \tau$

$\eta$

$\eta_{o}, \eta_{1}, \eta_{2}$

$\Gamma($.

$p_{k, \ell}$

$\Lambda$

$\lambda$

$\rho$

$\tau_{\ell}$

$\tau_{p}$

$\sigma_{k}$

$\varrho$

$\varepsilon$

$\xi_{\ell}$

\section{Explanation}

Kronecker Delta

The OFDM sub-carriers frequency spacing

The time duration of the bin

The average signal to noise plus interference ratio

Parameters to adjust the tightness of the approximation

The gamma function

An equiprobable \pm 1 R.V. to account for signal inversion

due to reflections

The cluster arrival rate

The ray arrival rate

A scaling factor that has the unit of time

Delay corresponding to the $\ell^{\text {th }}$ path

The UWB pulse shaping factor

The dB spread

The correlation coefficient between the two UWB pulses

Normalize the energy of the UWB pulses

The fading associated with the $\ell^{\text {th }}$ cluster 


\section{Chapter 1}

\section{Introduction}

\subsection{Research Motivation}

The appeal of UWB signalling for communications has been more recent and attracts a lot of attention globally. Several application areas have emerged for UWB, including: low rate with precise ranging wireless sensor networks, extremely high spatial resolution radar systems and high rate wireless USB-like communications between computer components or wireless links among components of entertainment systems (DVD player and TV) for short range data communication $(<10 \mathrm{~m})$.

Due to the restrictions imposed on the UWB transmitted power by the Federal Communications Commission (FCC) agency in addition to its wide operating spectrum, the UWB signal has very low spectral density so that it provides minimal interference with other narrowband communication systems, and makes it possible for UWB systems to share the already assigned spectrum resources with the existing narrowband wireless communication systems.

UWB communication may be achieved in the form of a single band impulse radio (IR) or a multi-band (orthogonal frequency division multiplexing (OFDM)) communication system. The multi-band OFDM based communication system combines the OFDM technique with the multi-band approach as the spectrum is divided into several sub-bands each with a bandwidth of $528 \mathrm{MHz}$. The information is interleaved across these sub-bands and then transmitted through multi-carrier OFDM technique. The impulse radio technique transmits pulses with the duration of sub-nanoseconds, which spread the energy from near DC to a few gigahertz without using a frequency carrier. In our research we focused on IR-UWB communication systems.

UWB may at times be vulnerable to interference from narrowband systems. To 
explain, due to the wide operating band of UWB impulse radio communication systems, many narrowband signals may exist in the UWB operating range, such as the standard IEEE802.11a wireless local area network (WLAN). These narrow band signals may exhibit higher power spectral density (PSD) levels compared to the PSD of UWB signals as seen by a UWB receiver, and so one would expect a degradation of the UWB bit error rate (BER) performance.

In order to enhance the UWB BER performance and achieve the goal of high data rate communication, it is important to mitigate the interference caused by high power narrowband signals. For these reasons the topic of narrowband interference (NBI) mitigation in UWB communication systems has attracted attention for the past few years and still attracts much interest.

The main motivation for the research work described in this thesis is to investigate the impact of high power NBI signals on IR-UWB communication systems in various UWB channel models and to examine methods to reduce the system BER degradation.

\subsection{Thesis Contributions}

The main contributions of this research can be summarized as follows:

1. An interference canceller technique is proposed to mitigate the cffect of NBI on IR-UWB communication systems, where the BER performance of such canceller scheme is presented using an analytical approach over various UWB channel models and validated with the aid of simulation.

2. It has been shown that in order to analyze the BER performance of the UWB communication system, while using the interference canceller scheme, we need to have analytic expressions for the average BER performance of the IR-UWB communication system in the presence of single and multiple tone interferers. Such formulas are required for various UWB channel models and have been analytically derived. Specifically:

- The average BER performance of a time hopping pulse position modulation (TH-PPM) UWB system in the presence of a single tone NBI signal in a Log-normal frequency selective fading channel is presented (see section $5.1)$. 
- The average BER performance of IR-UWB communication systems in the presence of NBI signal modeled as sum of $N$ tone interferers in a Lognormal flat fading channel is presented (see section 5.2).

- The average BER performance of a direct sequence binary phase shift keying (DS-BPSK) UWB system in the presence of NBI signal modeled as the standard IEEE802.11a WLAN in the IEEE802.15.3a UWB channel models is presented (see section 5.3).

3. The performance of the canceller scheme is investigated in different scenarios of operation such as the capability of the canceller scheme to mitigate the impact of multiple NBI signals (see section 6.1), the impact of symbol timing error (see section 6.2) and the impact of MAI under perfect and imperfect power control scenarios (see section 6.3). In addition, the average BER performance of the interference canceller scheme is compared with the performance enhancement obtained by using a notch filter specified with different quality factors. This comparison is done in two scenarios: perfectly tuned and de-tuned notch filter (see section 6.4).

\subsection{General Assumptions and Shortcomings}

Throughout our thesis we have used the following assumptions unless otherwise stated.

- For the desired UWB Signal:

The intended environment is the indoor UWB environment, the UWB signal is modeled as an IR-UWB signal ${ }^{1}$. The used UWB pulses are the second or the six derivative Gaussian pulses according to the NBI signal model and its corresponding center frequency. The UWB signal bandwidth is nearly $6 \mathrm{GHz}$ and the UWB signal data rate equals to $100 \mathrm{Mbps}$. We assumed that we operate in a perfect channel estimation, single user and synchronization scenarios.

- For the NBI signal:

The NBI signal is modeled as the standard OFDM based IEEE802.11a WLAN signal with center frequency equals to $5.745 \mathrm{GHz}$ and bandwidth equals to 20

\footnotetext{
${ }^{1}$ Modeled as a time hopping pulse position modulation or a direct sequence binary phase shift keying.
} 
$\mathrm{MHz}$. The NBI channel is modeled as a Rayleigh flat or a frequency selective fading channel. The NBI signal is characterized by a power spectral density which is much higher than the power spectral density of the UWB signal. We assumed that we operate in the presence of a single NBI signal.

The shortcomings in our research arise due to either the simplification of the analyses or due to the fact that in our proposal to mitigate the impact of high power NBI signal on the performance of IR-UWB communication system we focused on the NBI signal itself and on the performance of the narrowband receiver which demodulates the NBI signal ${ }^{2}$. To this end, the thesis shortcoming which will be covered in our future work can be summarized in the following points:

- The effect of UWB pulse shape imperfection was not addressed, we assumed that there is no distortion will occur to the UWB pulse before or during its reception.

- The impact of inter-symbol, inter-path and inter-chip interferences which had been addressed before [13] and [14] were not addressed.

- In our research we had investigated the capability of the canceller scheme to mitigate the impact of the presence of two WLAN NBI signals simultaneously, however the problem of in-band inter-modulation was not addressed.

\subsection{Thesis Organization}

The remainder of this research is organized as follows. Chapter 2 bricfly presents the basics of a UWB communication system and its associated data modulation and multiple access communication schemes. The UWB pulse shape, the used UWB propagation channel and its characteristics, in addition to a view on the global regulations on UWB are presented. This chapter also discusses the different sources of interference that could affect the performance of the UWB communication system. Finally, a literature review on the already used NBI suppression techniques is presented.

Chapter 3 presents the idea and the analytical representation of the proposed interference canceller scheme. Initially, the impact of UWB interference on the performance of a narrowband receiver is analyzed. A BER performance analysis of the

\footnotetext{
2 The idea and the analysis of the proposed interference canceller scheme will be emphasized in chapter 3 .
} 
IR-UWB communication system using the canceller scheme in AWGN channel environment is presented. The NBI signal is modeled as the standard IEEE802.11a signal. The BER performance of IR-UWB communication systems using the canceller scheme in a Nakagami-m flat fading channel environment is presented as well.

Chapter 4 presents the derived analytic formulas that investigate the performance of IR-UWB communication system in the presence of high power NBI signals in different fading channel environments. The analytic evaluation of the average BER performance of the interference canceller scheme in such fading channels is also done with the aid of these derived analytic formulas.

Chapter 5 presents the procedures taken to simulate the canceller scheme. The simulation results of the performance of the canceller scheme under various channel environments are also presented.

Chapter 6 presents an investigation to the performance effectiveness of the interference canceller scheme measured in different scenarios of operation. Its performance is also compared with the performance provided by the use of a notch filter.

Finally, chapter 7 concludes this thesis by outlining major findings. Some future work is also suggested. 


\section{Chapter 2}

\section{Literature Review}

\section{$2.1 \quad$ Introduction}

UWB technology was developed mainly for military purposes specially as a radar because UWB pulses could penetrate through walls and propagate beneath ground surfaces. Recently, UWB has been redirected to be used in other applications like precision localization, medical applications, target sensor data collection, tracking applications, and of course for communication applications. UWB technology is different from conventional narrowband wireless transmission technology. This difference arises from the fact that narrowband signals broadcast on dedicated frequencies, whereas UWB spreads signals across a very wide range of frequencies.

The most important concern in UWB system design is to minimize the possible interference to and from narrowband systems that are overlaid by UWB signals. Many systems such as the Global Positioning System (GPS), code division multiple access (CDMA), IEEE802.11b and IEEE802.11a WLANs etc., currently exist in or near the UWB frequency spectrum. Note that, the IEEE802.11b system operates at the unlicensed $2.4 \mathrm{GHz}$ band and the IEEE802.11a system operates at the unlicensed $5 \mathrm{GHz}$ band.

\subsection{UWB Communication Background}

The FCC gives definitions to UWB, wideband and narrowband signals and differentiate among them by means of what is called "signal fractional bandwidth". A narrowband signal has a fractional bandwidth less than $1 \%$, whereas a wideband has 
a fractional bandwidth in the range $1 \%$ to $20 \%$ [15]. A UWB is defined as any electromagnetic signal waveform that has an instantaneous fractional bandwidth greater than $20 \%$ with respect to its center frequency. The fractional bandwidth is given by:

$$
\text { Fractional } B . W .=\frac{\left(f_{H}-f_{L}\right)}{f_{c}}
$$

where $f_{H}$ and $f_{L}$ are the upper and lower $10 \mathrm{~dB}$ points of the transmitted signal power spectrum respectively. Also, according to the FCC UWB rulings, the signal is characterized as UWB if the signal bandwidth is $500 \mathrm{MHz}$ or more.

UWB has a number of features that make it attractive for consumer communications applications. In particular, UWB systems

1. have a noise-like signal spectrum.

2. can coexist with the operating narrowband signals.

3. are resistant to dense multi-path and jamming.

4. have very fine time-domain resolution allowing for location and tracking applications.

5. have the potential to support high data rates.

The low output power over a large bandwidth and the pseudo-random characteristics of the transmitted signal causes the UWB signal spectrum to be noise-like and hence the interception and detection of a UWB is a difficult task. hence, the low power, noise-like UWB signal can coexist with other narrowband operating services without causing a significant interference.

A UWB receiver can resolve multi-path components that are approximately "1/bandwidth" apart in the delay domain. This implies that the number of multipath components, distinguishable by the receiver, could be very high. This makes the UWB signal resistant to severe multi-path propagation and jamming/interference, but also makes the construction of UWB receivers more difficult. IR-UWB systems can exploit Rake receivers, which use separate correlators to receive the different multi-path components. 
The feature of high ratio of the transmitted UWB signal bandwidth to the information signal bandwidth makes it very attractive to be used as multiple access communication systems. In order to overcome catastrophic collisions in multiple access systems, UWB technology is accompanied with one of the spread spectrum techniques like time Hopping (TH) and direct sequence (DS) [16].

The transmission of very narrow pulses makes UWB systems potentially able to offer accurate timing precision. Together with the material penetration property, UWB signals can be used in short-range radar applications such as rescue and anticrime operations, as well as in the mining industry.

Finally, UWB signals can be transmitted with high data rates, which can be considered the most important aspect from user's and commercial manufacturer's point of view. Higher data rates bring the ability to establish new applications and devices to the market.

It is worth mentioning that, while current chip-sets are continually being improved, most UWB communication applications are targeting the range of $100 \mathrm{Mbps}$ to 500 Mbps [17]. This UWB data rate is roughly the equivalent of wired Ethernet to 500 times the speed of Bluetooth, around 50 times the speed of the $802.11 \mathrm{~b}$, or 10 times the 802.11a wireless local area network (LAN) standards.

Table 2.1 shows a comparison of indoor UWB data rate with other wireless standards.

Table 2.1: A comparison of indoor UWB data rate with other wireless standards.

\begin{tabular}{|c|c|}
\hline Standard & Data rate (Mbps) \\
\hline \hline Bluetooth (Version 1.2) & 1 \\
\hline IEEE802.11b & 11 \\
\hline IEEE802.11g & 20 \\
\hline IEEE802.11a & 54 \\
\hline UWB at distance 10m & 110 \\
\hline UWB at distance 4m & 200 \\
\hline UWB, USB 2.0 & 480 \\
\hline
\end{tabular}




\subsection{Data Modulation and Multiple Access Schemes}

\subsubsection{Data Modulation Methods}

There are several basic methods of modulation used with UWB systems, which include both orthogonal and antipodal schemes. Modulation schemes can be classified into two main categories: time-based represented by pulse position modulation (PPM) and shape-based represented by on-off keying, pulse amplitude modulation, Bi-phase modulation and pulse shape modulation.

\subsubsection{On-Off Keying}

The first modulation scheme under the shape-based category is called the on-off keying (OOK) scheme, where the absence or the presence of a pulse signifies the digital information of 0 or 1 respectively. We can define the transmitted binary modulated signal $S_{u}(t)$ as [18].

$$
S_{u}(t)=d_{j} \times p(t)
$$

where $p(t)$ is the pulse waveform and $j \in 0,1$.

$d_{j}$ can be defined as

$$
d_{j}= \begin{cases}0, & \mathrm{j}=0 \\ 1, & \mathrm{j}=1\end{cases}
$$

The presence of multi-path is considered the major problem of OOK, because echoes of the original or other pulses make it difficult to determine the absence of a pulse. Figure 2.1 depicts the OOK modulation scheme for "0" and "1" bits.

\subsubsection{Pulse Amplitude Modulation}

The second modulation scheme is called pulse amplitude modulation (PAM) where the amplitude of the pulse varies to contain digital information, where $d_{j}$ in equation (2.2) can take on positive values greater than zero. For 2 levels PAM: 


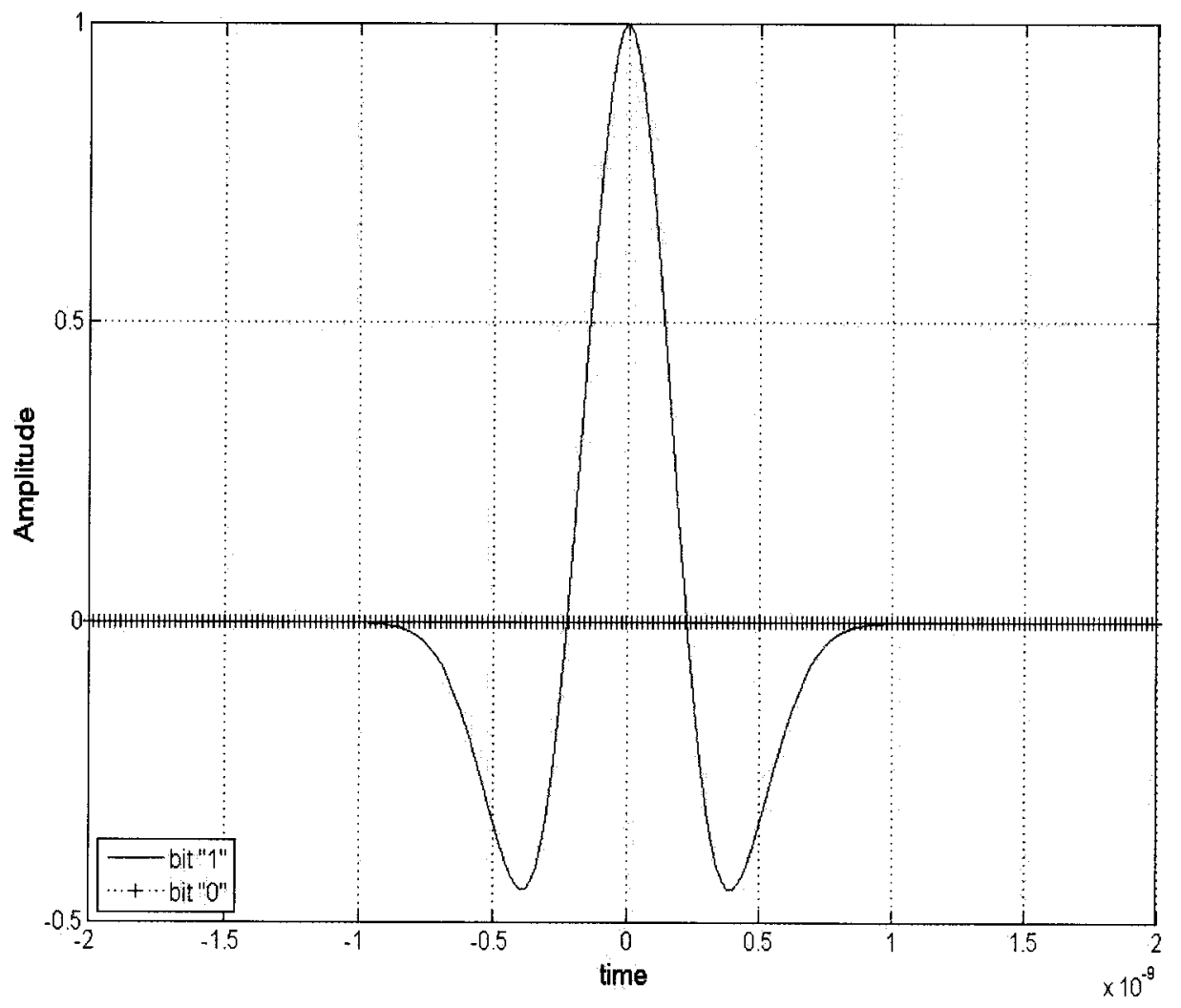

Figure 2.1: OOK for "0" and "1" bits 


$$
d_{j}= \begin{cases}1, & j=0 \\ 2, & j=1\end{cases}
$$

Figure 2.2 depicts the 2-PAM modulation scheme for "0" and "1" bits.

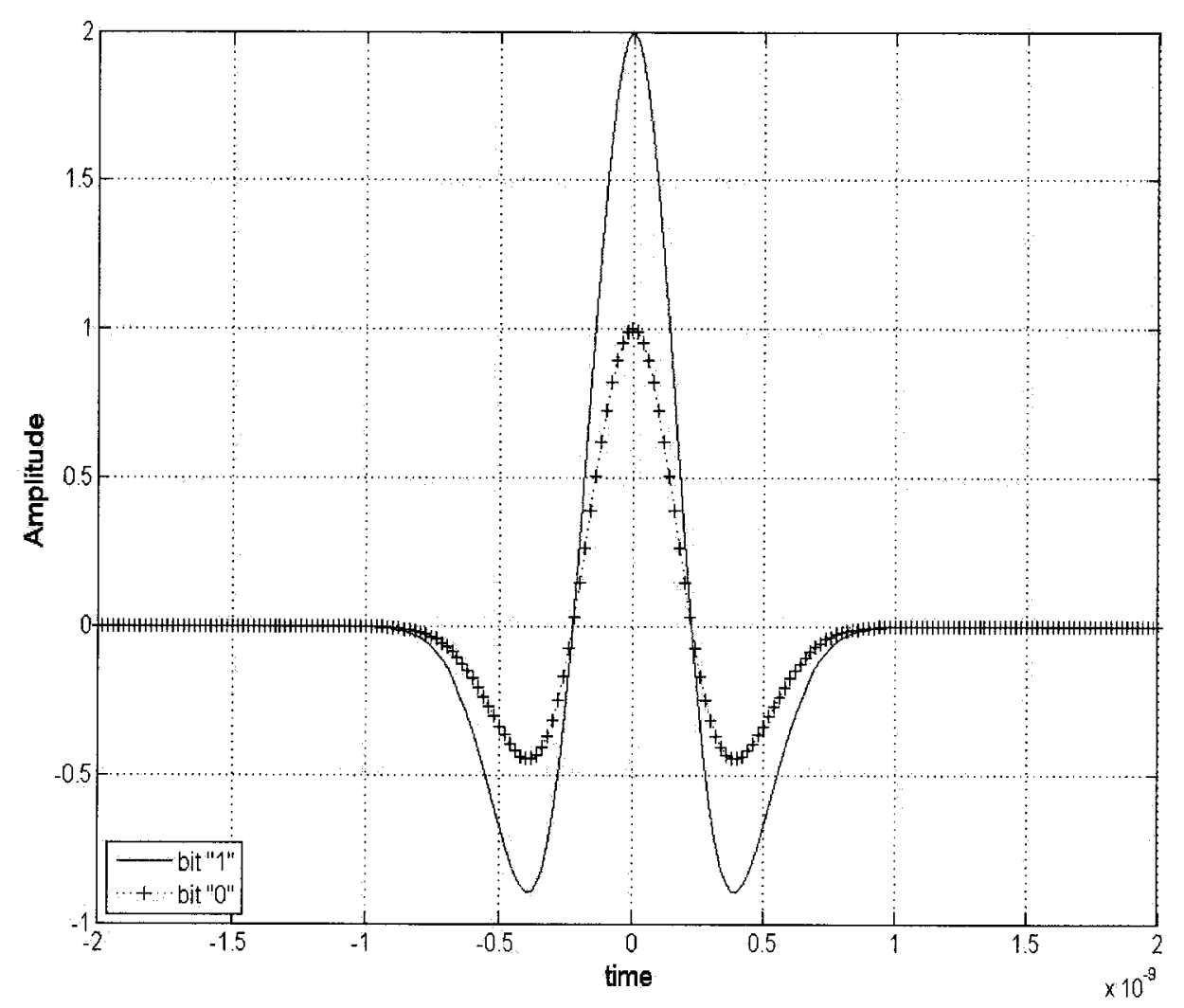

Figure 2.2: 2-PAM for " 0 " and "1" bits

Generally, PAM is not the preferred modulation scheme for most short range communication, because an amplitude-modulated signal which has a smaller amplitude is more susceptible to noise interference than its larger amplitude counterpart. Furthermore, the higher amplitude pulse requires more power to be transmitted .

\subsubsection{Bi-Phase Modulation}

It can be defined as a kind of shape modulation presented using the two antipodal pulses. It is simply interpreted as the inversion of a particular pulse shape. 
$d_{j}$ can be written as

$$
d_{j}= \begin{cases}-1, & \mathrm{j}=0 \\ +1, & \mathrm{j}=1 .\end{cases}
$$

Figure 2.3 depicts the Bi-phase modulation scheme for "0" and "1" bits.

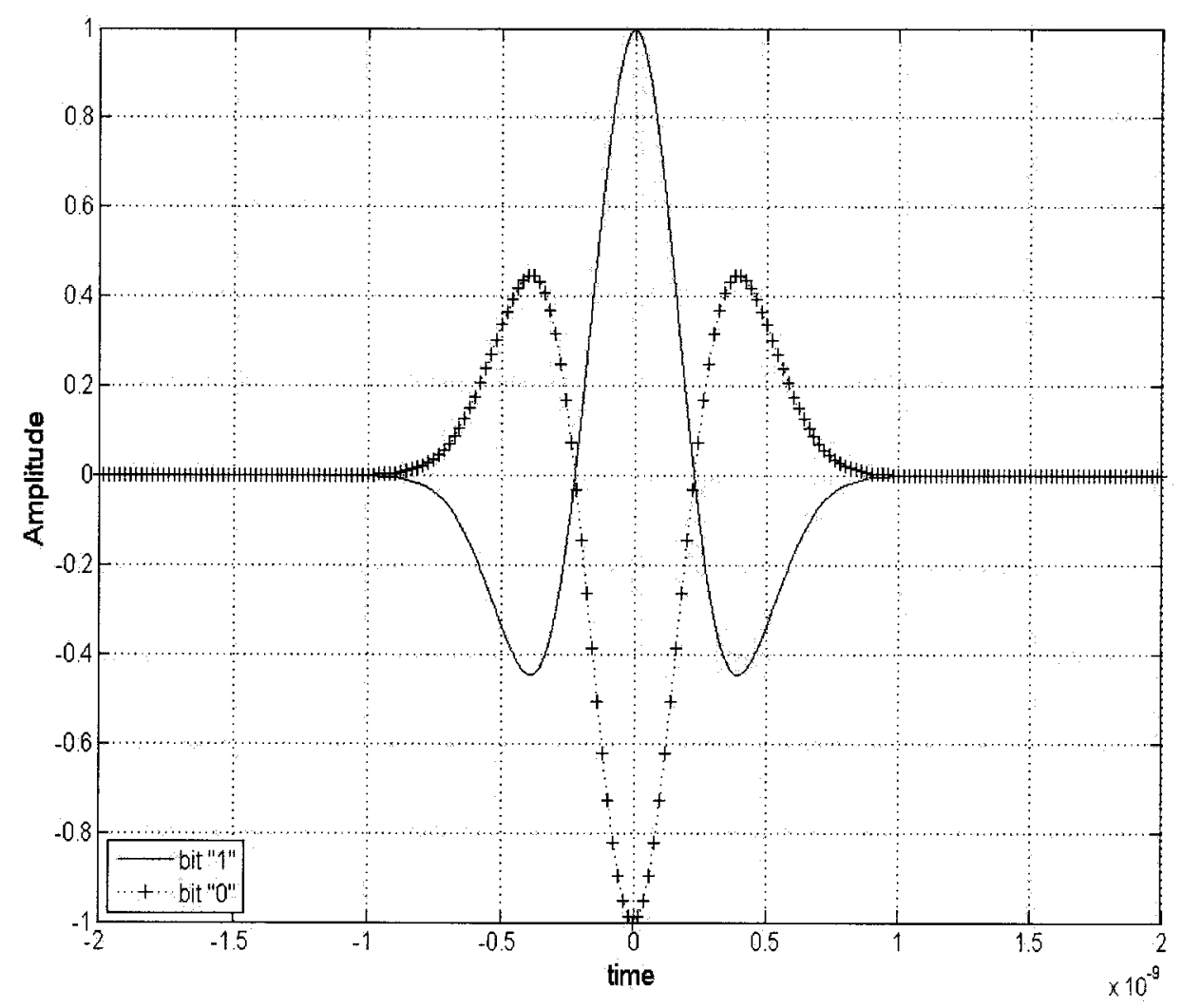

Figure 2.3: Bi-phase modulation for "0" and "1" bits

\subsubsection{Pulse Shape Modulation}

In pulse shape modulation (PSM) technique different pulse shapes are used to represent information bits. This requires a suitable set of pulses to be used. It is based mainly on using orthogonal pulse waveforms to represent "0" and "1". The transmitted signal can be written as 


$$
S_{u}(t)=\left(1-d_{j}\right) \times p_{0}(t)+d_{j} \times p_{1}(t)
$$

where $d_{j} \in 0,1$ and $p_{0}(t), p_{1}(t)$ are orthogonal pulses.

Wavelets [19], modified Hermite polynomial functions (MHPF) [20], and prolate spheroidal wave functions (PSWF) [19] have been proposed as pulse sets for PSM systems and all exhibit orthogonal properties. Optimum detection at the receiver is achieved by the orthogonality of these pulses, also the ability to assign each user with its own orthogonal pulse which leads to achieving multiple access property.

\subsubsection{Pulse Position Modulation}

In pulse position modulation (PPM), the chosen bit to be transmitted influences the position of the UWB pulse. That means the pulse representing the information bit " 1 " is delayed in time by the amount $\delta$, whereas the pulse representing the information bit " 0 " is not shifted in time [16]. The signal can be represented as follows:

$$
S_{u}(t)=p\left(t-\delta d_{j}\right)
$$

where $d_{j}$ takes the following values

$$
d_{j}= \begin{cases}0, & j=0 \\ 1, & j=1\end{cases}
$$

The value of $\delta$ may be chosen according to the autocorrelation characteristics of the pulse. The autocorrelation function is defined as

$$
R(\tau)=\int_{-\infty}^{\infty} p(t) \times p(t+\tau) d \tau
$$

The optimum value of the shift $\delta$ is the one that satisfies the following equation [21]

$$
R\left(\delta_{\text {opt }}\right)=\int_{-\infty}^{\infty} p(\tau) \times p\left(\tau+\delta_{\text {opt }}\right) d \tau=0
$$

The advantages of PPM mainly arise from its simplicity and the ease with which the delay may be controlled. If we make a comparison between PPM and bi-phase modulation we will notice that the latter is an antipodal modulation method, whereas PPM, when separated by one pulse width delay for each pulse position, is an orthogonal modulation method so, bi phase modulation achieves 3-dB gain in power efficiency 


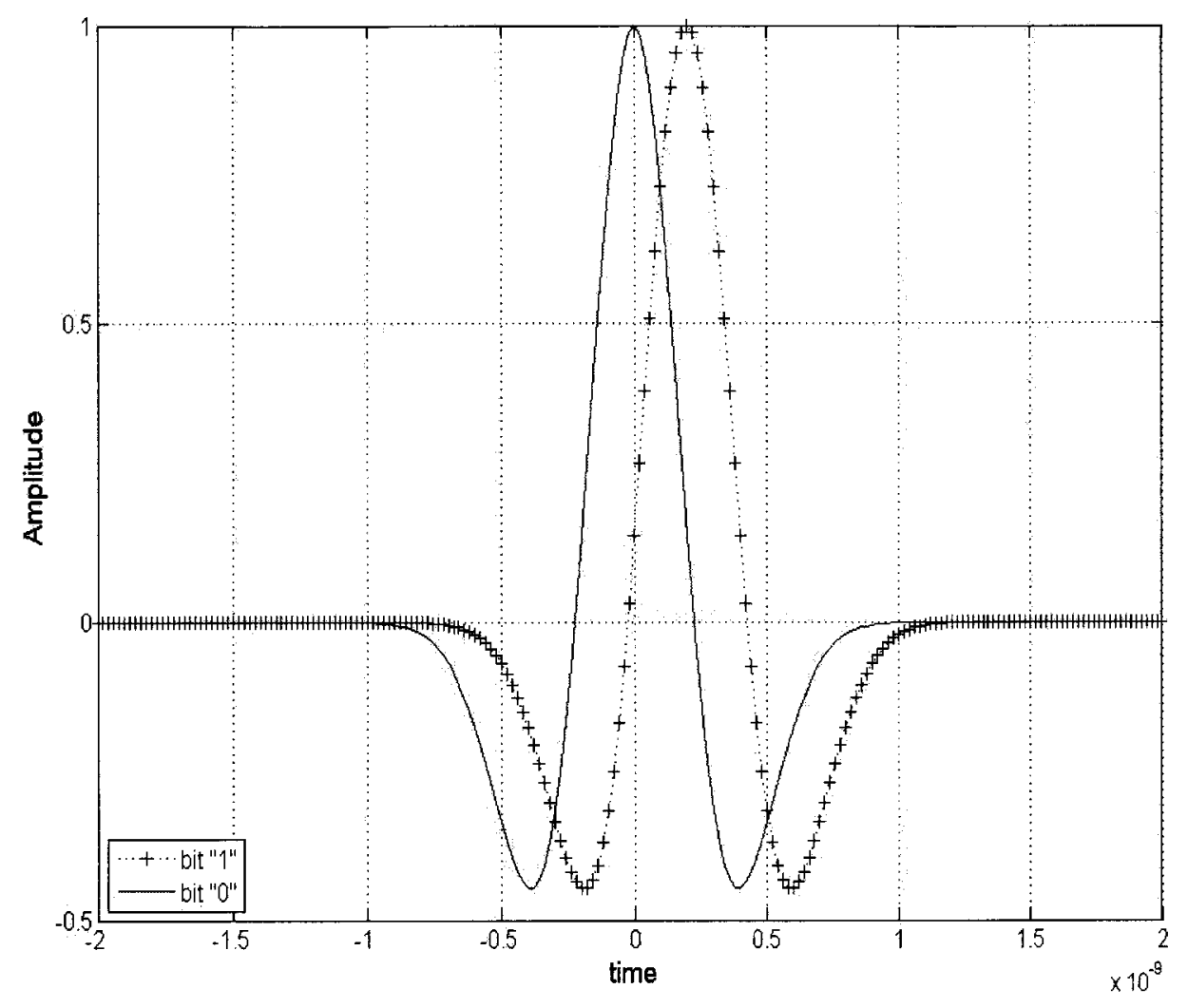

Figure 2.4: PPM modulation scheme for "0" and " 1 " bits

compared to PPM.

Figure 2.4 depicts the PPM modulation scheme for " 0 " and " 1 " bits, where bit " 1 " is delayed by $\delta$ whereas bit " 0 " is set at the nominal time.

\subsubsection{Multiple Access Methods}

In the following we will briefly introduce the main two techniques used as multiple access techniques in UWB systems. 


\subsubsection{Direct Sequence UWB}

DS-UWB scheme uses sequences of UWB pulses ${ }^{1}$, where a user in the system is assigned a unique pseudo random code sequence that controls the randomness of the UWB pulses. A data bit is then used to modulate this sequence of UWB pulses.

For the pulse amplitude modulation and on-off keying modulation, the data signal for the $k^{t h}$ user can be written as

$$
S^{(k)}(t)=\sum_{j=-\infty}^{\infty} \sum_{n=0}^{N_{c}-1} d_{j}^{(k)} c_{n}^{(k)} p\left(t-j T_{b}-n T_{c}\right)
$$

where for a DS-UWB system:

- $\mathrm{p}(\mathrm{t})$ is the shape of the transmitted pulse with pulse width $T_{m}$.

- $T_{c}$ is the DS chip duration.

- $c_{n}$ is the DS spreading code sequence.

- $N_{c}$ is the number of chips per information bit.

- $T_{b}$ is the bit duration.

- $d_{j}$ is the transmitted $j^{\text {th }}$ binary data bit and composed of equally likely symbols (or bits).

The DS code is used to change the polarity of the pulse in one information bit. The pseudo random sequence has values " $-1,+1$ " and the data bit duration $T_{b}=N_{c} T_{c}$.

Figure 2.5 shows a sample of the DS-BPSK-UWB signal waveform, where $N_{c}=5$ chip/bit.

\subsubsection{Time Hopping UWB}

By combining PPM technique along with TH multiple access method we will get a simple UWB transmitter system. This system is the most common in the literature. This approach allows the use of a matched filter, while the optimum time shift depends on the cross-correlation properties of the pulses used. A single template pulse is required for reception, and the complexity of this system resides in providing accurate

\footnotetext{
${ }^{1}$ In UWB systems, the very narrow pulse waveform takes the role of the chip in a spread spectrum system.
} 


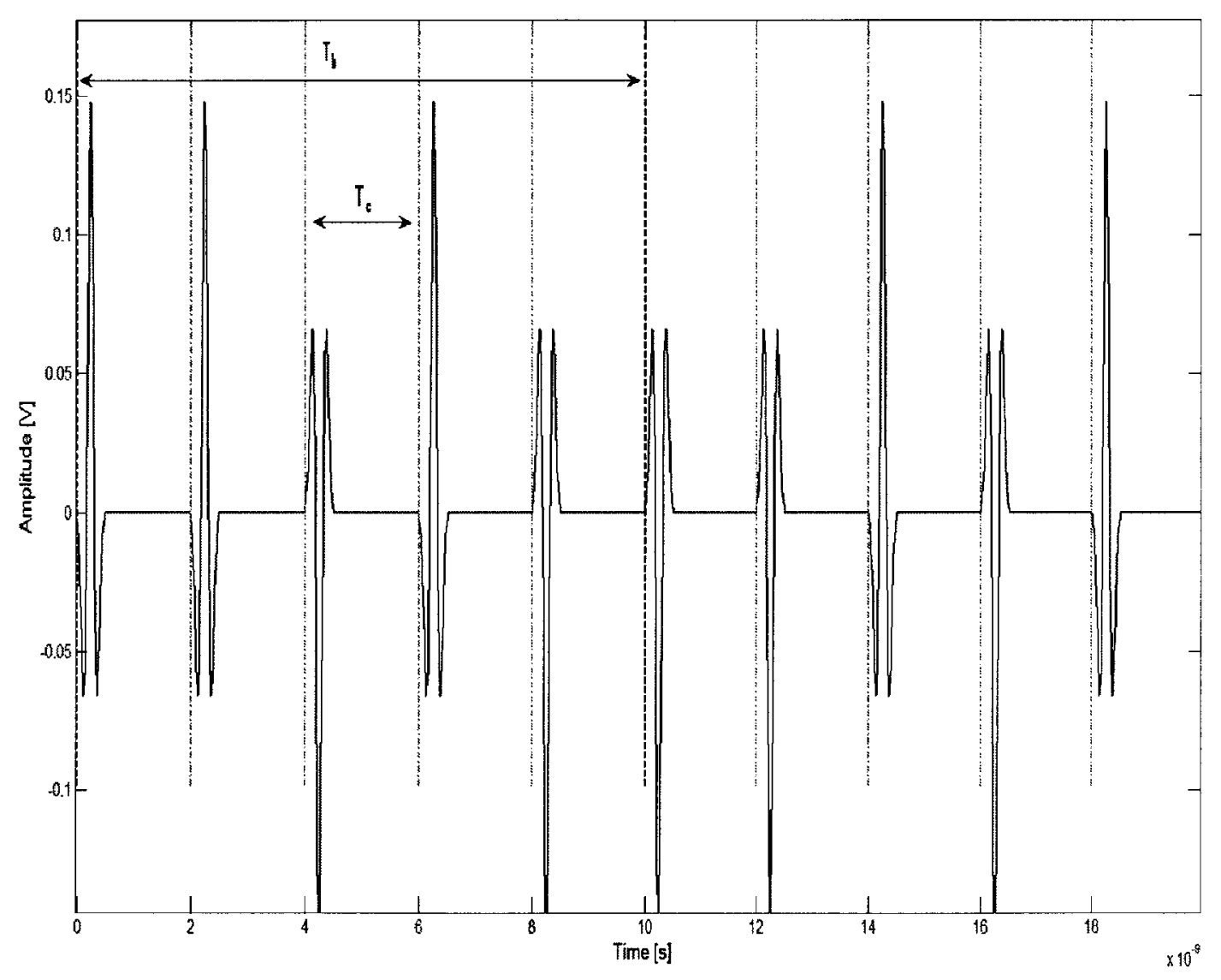

Figure 2.5: Sample of UWB DS-BPSK Waveform for two bits ( 1,0 respectively) $\left(N_{c}=5 \mathrm{chip} / \mathrm{bit}\right)$ 
timing for the generation of the transmitted sequence and subsequent reception. The modulated data stream of the TH-PPM system for the $k^{\text {th }}$ user can be written as follows

$$
S^{(k)}(t)=\sum_{j=-\infty}^{\infty} \sum_{n=0}^{N_{s}-1} p\left(t-j T_{b}-n T_{f}-c_{j}^{(k)} T_{c}-\delta d_{j}^{(k)}\right)
$$

where

- $\mathrm{p}(\mathrm{t})$ is the shape of the transmitted pulse with pulse width $T_{m}$.

- $T_{c}$ is the TH chip width.

- $c_{j}$ is the TH code $\in\left\{0,1, \ldots, N_{h}-1\right\}$, such that an additional time shift of $c_{j} T_{c}$ is introduced when the $j^{\text {th }}$ pulse is transmitted.

- $N_{s}$ is the number of pulses transmitted per bit ${ }^{2}$.

- $T_{f}$ is the TH frame duration $\left(T_{f}=N_{h} T_{c}\right)$, where the bit duration can be represented as $T_{b}=N_{s} T_{f}$.

- $d_{\left\lfloor j / N_{s}\right\rfloor}$ is the $j^{\text {th }}$ binary data bit transmitted and composed of equally likely symbols (or bits).

- Finally, $\delta$ is the modulation index (the time shift added to a pulse with an optimal value of $20 \%$ of a pulse width) [22].

For PAM the information signal can be writhen as

$$
S^{(k)}(t)=\sum_{j=-\infty}^{\infty} \sum_{n=0}^{N_{s}-1} d_{j}^{(k)} \times p\left(t-j T_{b}-n T_{f}-c_{j}^{(k)} T_{c}\right)
$$

while for pulse shaping modulation, it can be written as

$$
S^{(k)}(t)=\sum_{j=-\infty}^{\infty} \sum_{n=0}^{N_{s}-1} p_{d_{j}^{(k)}}\left(t-j T_{b}-n T_{f}-c_{j}^{(k)} T_{c}\right)
$$

Figure 2.6 shows a sample of the TH-PPM-UWB signal waveform, where $N_{s}=5$ pulse/bit, $N_{h}=3$.

\footnotetext{
${ }^{2}$ Generated by the Code Repetition Coder (see chapter 4).
} 


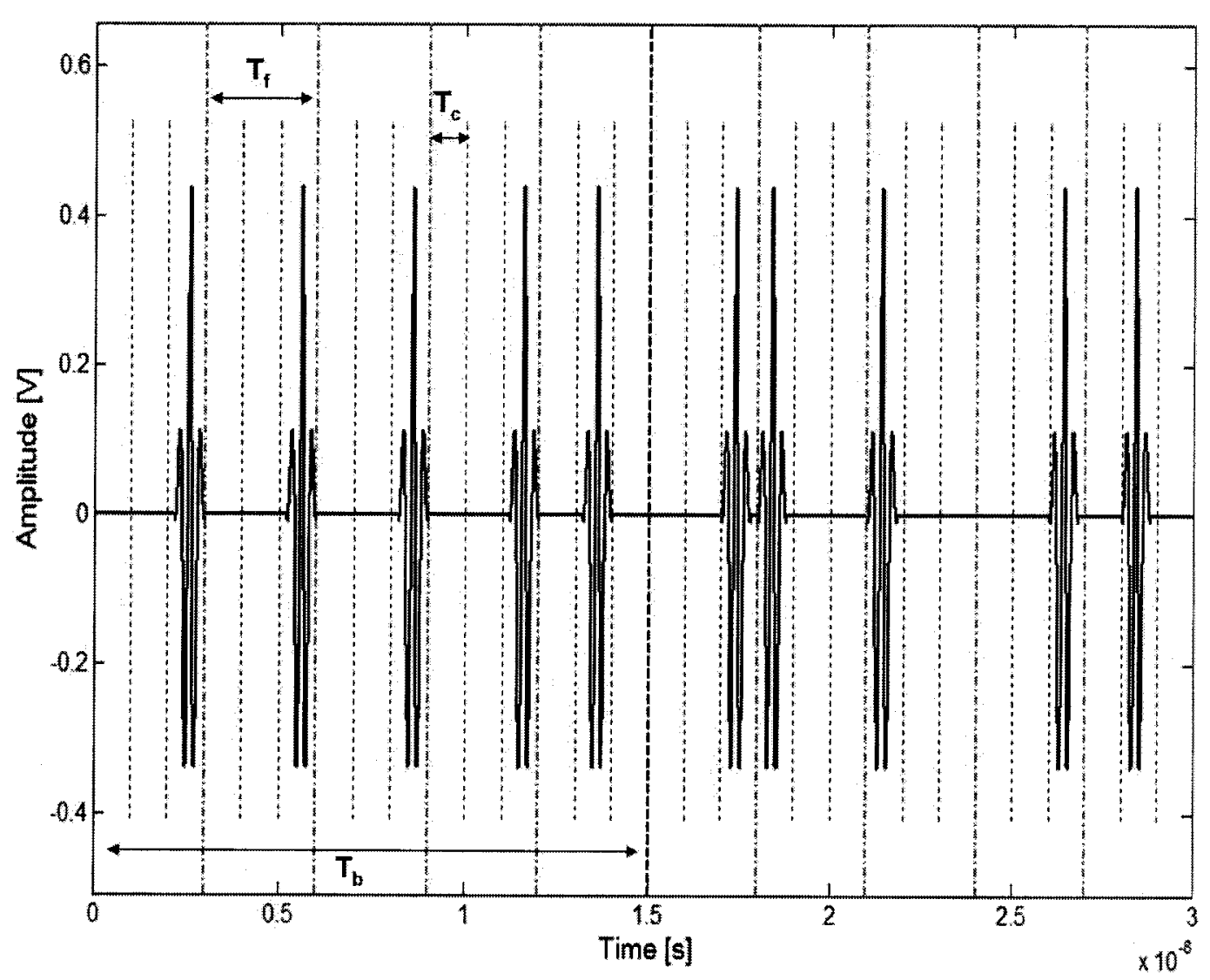

Figure 2.6: Sample of UWB TH-PPM Waveform for two bits (1,0 respectively) ( $N_{s}$ $=5$ pulse $/$ bit,$N_{h}=3$ ) 


\subsection{UWB Pulse Shape}

In a UWB system, the choice of the pulse shape affects the choice of the receiver bandwidth, the bit error rate performance and the performance in multi-path propagation environments. A variety of pulse shapes has been proposed for UWB impulse radio systems. Examples of pulse shapes include the Gaussian pulse, Gaussian mono-cycles, orthogonal modified Hermite pulses, orthogonal prolate spheroidal wave functions, and the Manchester mono-cycle [23] and [24].

In our work, we focused on using the Gaussian pulse and its derivatives. The Gaussian pulse has the form

$$
p_{o}(t)=\exp \left[-2 \pi\left(\frac{t}{\tau_{p}}\right)^{2}\right]
$$

where $\tau_{p}$, is the pulse shaping factor.

The time and the frequency representations of the $n^{\text {th }}$-order Gaussian mono-cycle are given by [25]

$$
\begin{gathered}
p_{n}(t)=(-1)^{\lfloor(3 n+1 / 2)\rfloor} n !(\pi)^{-1 / 4} e^{-a t^{2}} \times \sum_{k=0}^{\lfloor n / 2\rfloor} \frac{(-1)^{k} 2^{n+1 / 4-2 k} a^{n / 2+1 / 4-k} t^{n-2 k}}{(n-2 k) ! k ! \sqrt{(2 n-1) ! !}} \\
P_{n}(f)=\frac{(-1)^{n} i^{n^{2}}(2 \pi)^{n+1 / 4} a^{-(n / 2+1 / 4)}}{\sqrt{(2 n-1) ! !}} f^{n} \exp \left(-\pi^{2} f^{2} / a\right)
\end{gathered}
$$

where $\mathrm{a}=\frac{1}{2 \tau_{p}^{2}}$, and $(2 n-1) ! !=(2 \mathrm{n}-1) \times(2 \mathrm{n}-3) \times \ldots \times 3 \times 1$.

Two derivatives of the Gaussian mono-cycle have been used in our research, the second and the six derivative to comply with the FCC spectral mask.

A second derivative Gaussian pulse can be written as [26]

$$
p(t)=\varepsilon\left[1-4 \pi\left(\frac{t}{\tau_{p}}\right)^{2}\right] \exp \left[-2 \pi\left(\frac{t}{\tau_{p}}\right)^{2}\right]
$$

For a Gaussian mono-cycle with energy $1 / N_{s}, \varepsilon$ can be written as $\left[\varepsilon=\sqrt{\frac{8}{3 N_{s} \tau_{p}}}\right][21]$. The six derivative Gaussian pulse can be written as [27]

$$
p(t)=\sqrt{\frac{640}{231 N_{s} \tau_{p}}}\left[1-12 \pi\left(\frac{t}{\tau_{p}}\right)^{2}+16 \pi^{2}\left(\frac{t}{\tau_{p}}\right)^{4}-\frac{64}{15} \pi^{3}\left(\frac{t}{\tau_{p}}\right)^{6}\right] \exp \left[-2 \pi\left(\frac{t}{\tau_{p}}\right)^{2}\right]
$$


It was reported in $[20]^{3}$ that the differentiation of the Gaussian pulse influences the UWB energy spectral density as well both peak energy frequency and the bandwidth of the UWB pulse ${ }^{4}$. The peak energy frequency is given by

$$
f_{\text {peak }}=\sqrt{n} \frac{1}{\tau_{p} \sqrt{\pi}}
$$

Figure 2.7 depicts the relation between the peak energy frequency $f_{\text {peak }}$, the order of differentiation $\mathrm{n}$, and the pulse shaping factor $\tau_{p}$. While figure 2.8 depicts the relation

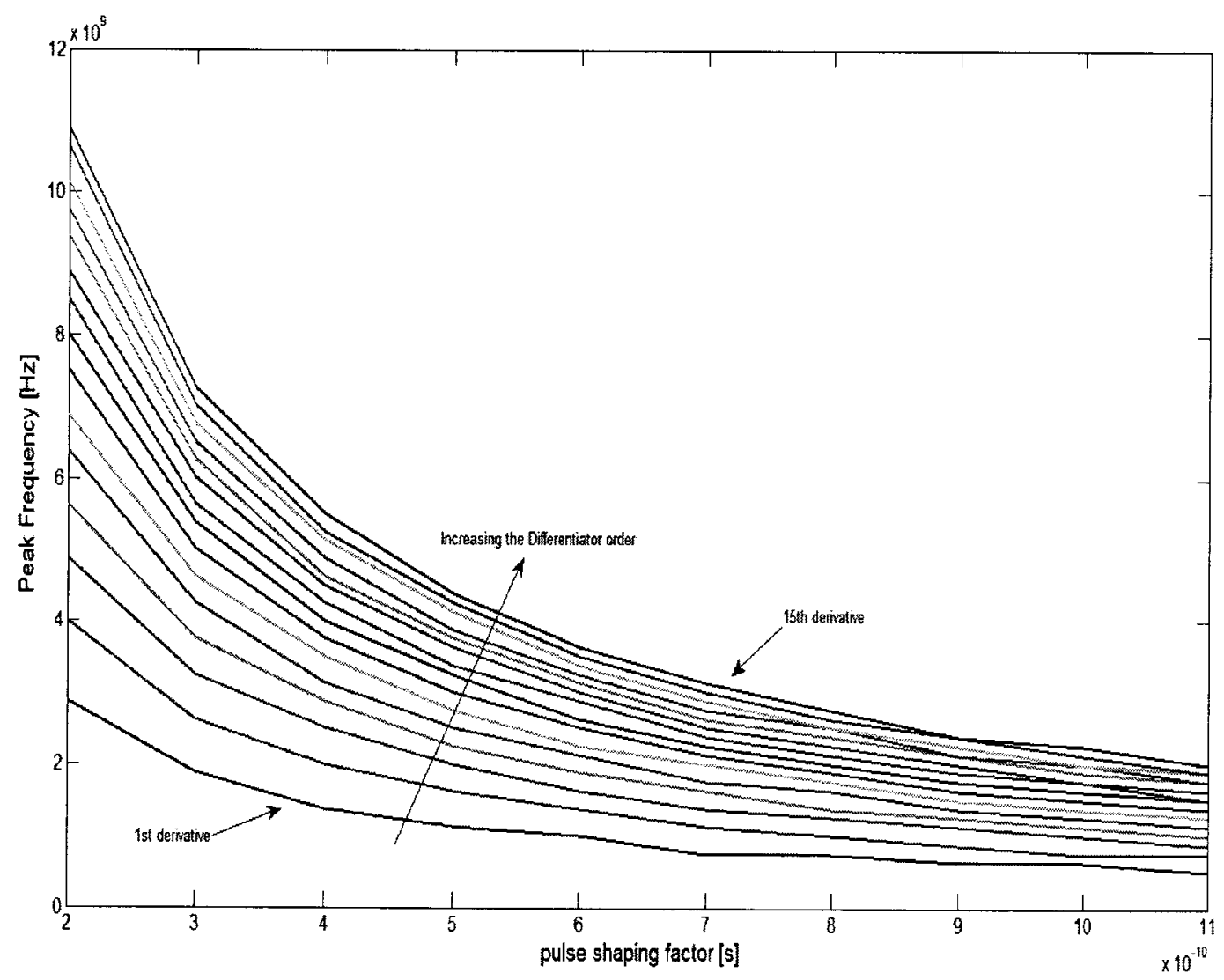

Figure 2.7: The impact of varying the pulse shaping factor on the peak energy frequency value for the first 15 derivatives of the UWB Gaussian pulse.

between the $-10 \mathrm{~dB}$ bandwidth, the order of differentiation $\mathrm{n}$, and the pulse shaping factor $\tau_{p}$. It can be seen that the differentiation process can be considered as a mean

\footnotetext{
${ }^{3}$ Chapter one page 12.

4 The UWB $-10 \mathrm{~dB}$ bandwidth increases with increasing the differentiator order.
} 


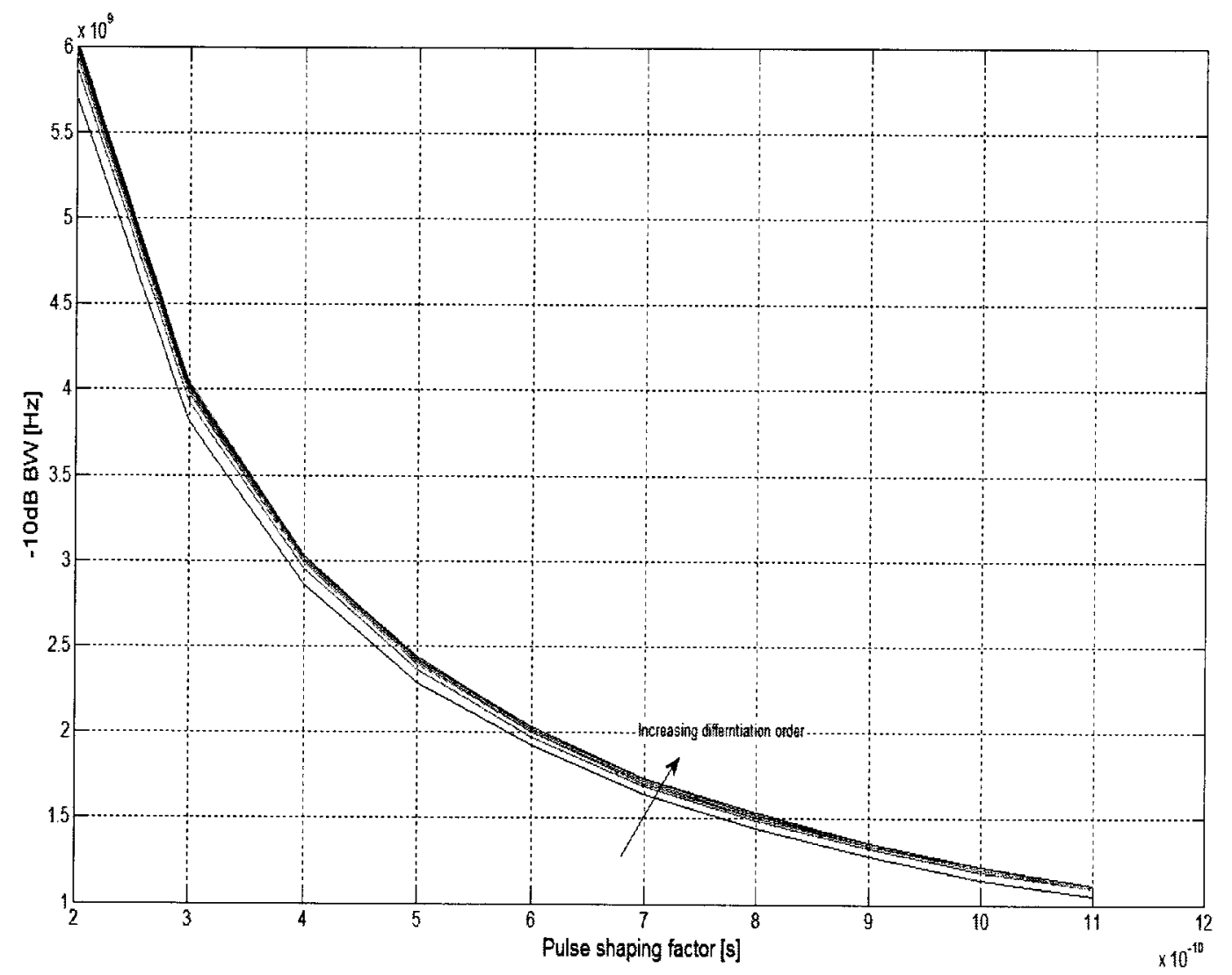

Figure 2.8: The impact of varying the pulse shaping factor on the $-10 \mathrm{~dB}$ bandwidth value for the first 15 derivatives of the UWB Gaussian pulse. 
to move energy to high frequency bands and to comply with the FCC part 15 limit. Table 2.2 depicts the $-10 \mathrm{~dB}$ bandwidth relation with the Gaussian pulse order of differentiation $\mathrm{n}$ and the Gaussian pulse standard deviation $\sigma$ [1]. The pulse shaping factor $\tau_{p}=2 \sqrt{\pi} \sigma$.

Table 2.2: The $-10 \mathrm{~dB}$ bandwidth relation with the Gaussian pulse order of differentiation and the Gaussian pulse standard deviation [1].

\begin{tabular}{|c|c|}
\hline $\mathrm{n}$ & $-10 \mathrm{~dB}$ B.W. \\
\hline \hline 1 & $0.32 / \sigma$ \\
\hline 2 & $0.33 / \sigma$ \\
\hline 3 & $0.33 / \sigma$ \\
\hline 4 & $0.34 / \sigma$ \\
\hline 5 & $0.34 / \sigma$ \\
\hline
\end{tabular}

\subsection{UWB Propagation Channel}

The time resolution of the UWB channel is in the order of a nanosecond based on several GHz bandwidth of a UWB signal. Various fading models have been presented based on significant data collection efforts such as [28]:

- Nakagami distribution has been suggested by [29] for bins with large delays.

- Rice distribution has been suggested by [30] for bins with small delays in Lineof-sight (LOS) situations. It is shown in [31] that it best fit measurement results in an office environment.

- Log-normal distribution has been suggested by [32]. It is shown that this approach has the advantage that the fading statistics of the small-scale and the large-scale variations have the same form.

In most indoor environments, objects are not distributed uniformly in space but rather are clustered. The clustering of objects can be, in a first approximation, translated into clustering of multi-path components. To this end, the IEEE802.15.3a Working Group for Wireless Personal Area Networks, and its channel modeling subcommittee 
proposed a channel model for UWB systems in 2003 [32]. This channel model is based on the clustering phenomenon observed in several channel measurements.

The multi-path indoor fading model is a modified version of the popular Saleh-Valenzuela (S-V) channel model [33], where multi-path components arrive in clusters. A Log-normal distribution is employed rather than a Rayleigh distribution for the multi-path gain magnitude, since UWB observations show that the Log-normal distribution seems to better fit the measurement data. In addition, independent fading is assumed for each cluster as well as for each ray within the cluster.

The multi-path model consists of the following discrete time impulse response [28]:

$$
h(t)=X \sum_{\ell=0}^{L} \sum_{k=0}^{K} \alpha_{k, \ell} \delta\left(t-T_{\ell}-\tau_{k, \ell}\right)
$$

where $\alpha_{k, \ell}$ is the multi-path gain coefficient for cluster $\ell$ and ray $\mathrm{k}, T_{\ell}$ is the delay of the $\ell^{\text {th }}$ cluster, $\tau_{k, \ell}$ is the delay of the $k^{\text {th }}$ ray relative to the arrival time of the $\ell^{\text {th }}$ cluster, and $\mathrm{X}$ represents the Log-normal shadowing. The number of clusters and rays in the multi-path fading model is denoted as $\mathrm{L}$ and $\mathrm{K}$, respectively, which are fixed for a given model.

By definition, we have $\tau_{0, \ell}=0$. The distribution of cluster-arrival time and the ray-arrival time are given by

$$
\begin{aligned}
p\left(T_{\ell} / T_{\ell-1}\right) & =\Lambda \exp \left[-\Lambda\left(T_{\ell}-T_{\ell-1}\right)\right], \ell>0 \\
p\left(\tau_{k, \ell} / \tau_{k-1, \ell}\right) & =\lambda \exp \left[-\lambda\left(\tau_{k, \ell}-\tau_{k-1, \ell}\right)\right], k>0
\end{aligned}
$$

where $\Lambda$ is the cluster arrival rate and $\lambda$ is the ray arrival rate ${ }^{5}$.

The channel coefficients are defined as follows:

$$
\begin{gathered}
\alpha_{k, \ell}=p_{k, \ell} \xi_{\ell} \beta_{k, \ell} \\
\left|\xi_{\ell} \beta_{k, \ell}\right|=10^{\left(\mu_{k, \ell}+n_{1}+n_{2}\right) / 20}
\end{gathered}
$$

\footnotetext{
${ }^{5}$ The arrival rate of paths within each cluster.
} 
where $n_{1} \propto \operatorname{Normal}\left(0, \sigma_{1}^{2}\right)$ and $n_{2} \propto \operatorname{Normal}\left(0, \sigma_{2}^{2}\right)$ are independent and correspond to the fading on each cluster and ray respectively. $\sigma_{1}$ and $\sigma_{2}$ are the standard deviation of cluster and ray Log-normal fading terms respectively $(\mathrm{dB})$.

$$
E\left[\left|\xi_{\ell} \beta_{k, \ell}\right|^{2}\right]=\left|\beta_{0,0}\right|^{2} e^{-T_{\ell} / \Gamma} e^{-\tau_{k, \ell} / \gamma}
$$

$\left|\beta_{0,0}\right|^{2}$ is the mean energy of the first path of the first cluster, and $p_{k, \ell}$ is equiprobable \pm 1 to account for signal inversion due to reflections. $\xi_{\ell}$ is the fading associated with the $\ell^{\text {th }}$ cluster, and $\beta_{k, \ell}$ is the fading associated with the $k^{\text {th }}$ ray of the $\ell^{\text {th }}$ cluster. $\mu_{k, \ell}$ is given by [2]

$$
\mu_{k, \ell}=\frac{10 \ln \left(\left|\beta_{0,0}\right|^{2}\right)-10 T_{\ell} / \Gamma-10 \tau_{k, \ell} / \gamma}{\ln (10)}-\frac{\left(\sigma_{1}^{2}+\sigma_{2}^{2}\right) \ln (10)}{20}
$$

where $\Gamma$ and $\gamma$ are the cluster and ray decay factors respectively.

The log-normal shadowing is characterized as

$$
20 \log _{10}(X) \propto \operatorname{Normal}\left(0, \sigma_{x}^{2}\right)
$$

where $\sigma_{x}$ is the standard deviation of Log-normal shadowing term for total multi-path realization $(\mathrm{dB})$.

Based on the average distance between the transmitter and the receiver and whether there is a LOS component or not, four different channel implementations are suggested as follows:

- CM1: LOS model for 0-4m.

- CM2: None-Line-of-Sight (NLOS) model for 0-4m.

- CM3: NLOS model for $4-10 \mathrm{~m}$.

- CM4: Extreme (very high multi-path) NLOS model 4-10m.

Table 2.3 depicts the IEEE suggestion to the set of values for the IEEE UWB channel models parameter settings $[2]^{6}$. These values were tuned to fit some of the measurement data submitted to the IEEE.

\footnotetext{
${ }^{6}$ Appendix B, Page (225)
} 
Table 2.3: Parameter Settings for the IEEE UWB Channel Model [2].

\begin{tabular}{|c|ccccccc|}
\hline Scenario & $\Lambda(1 / n s)$ & $\lambda(1 / n s)$ & $\Gamma$ & $\gamma$ & $\sigma_{x}(d B)$ & $\sigma_{1}(d B)$ & $\sigma_{2}(d B)$ \\
\hline \hline CM1 (LOS 0-4m) & 0.0233 & 2.5 & 7.1 & 4.3 & 3 & 3.3941 & 3.3941 \\
\hline CM2 (NLOS 0-4m) & 0.4 & 0.5 & 5.5 & 6.7 & 3 & 3.3941 & 3.3941 \\
\hline CM3 (NLOS 4-10m) & 0.0667 & 2.1 & 14 & 7.9 & 3 & 3.3941 & 3.3941 \\
\hline CM4 (Extreme NLOS) & 0.0667 & 2.1 & 24 & 12 & 3 & 3.3941 & 3.3941 \\
\hline
\end{tabular}

\subsection{Global Regulations on UWB}

\subsubsection{UWB regulations in the United States}

One of the important issues for UWB communications is frequency allocation. The FCC specifies a set of rules to control harmful interference from UWB devices while allowing emission between 3.1 and $10.6 \mathrm{GHz}$. The UWB Emission limits are given in terms of equivalent isotropically radiated power (EIRP). According to the FCC regulations, the maximum EIRP in any direction should not exceed $-41.3 \mathrm{dBm} / \mathrm{MHz}^{7}$.

Note that, the FCC emission limits for UWB indoor and outdoor communication systems differ as depicted in figure 2.9 .

\subsubsection{UWB regulations in the European Union}

In Europe, the organizations involved in the regulation of UWB are ETSI (European Technical Standard Institute) and CEPT (European Conference of Postal and Telecommunications Administration). They made a final decision at the beginning of 2007 for UWB regulations. The spectrum mask imposed by the European Commission is shown in figure 2.10 for indoor UWB communications. By comparing the European and FCC spectral masks, it can be noted that the European approach to UWB emission is more restrictive than FCC, and only in the band $6-8.5 \mathrm{GHz}$ does it have the same emission level as the FCC.

\footnotetext{
7 This emission limit is the part 15 limit, which is identical to the limit for unintentional radiators.
} 


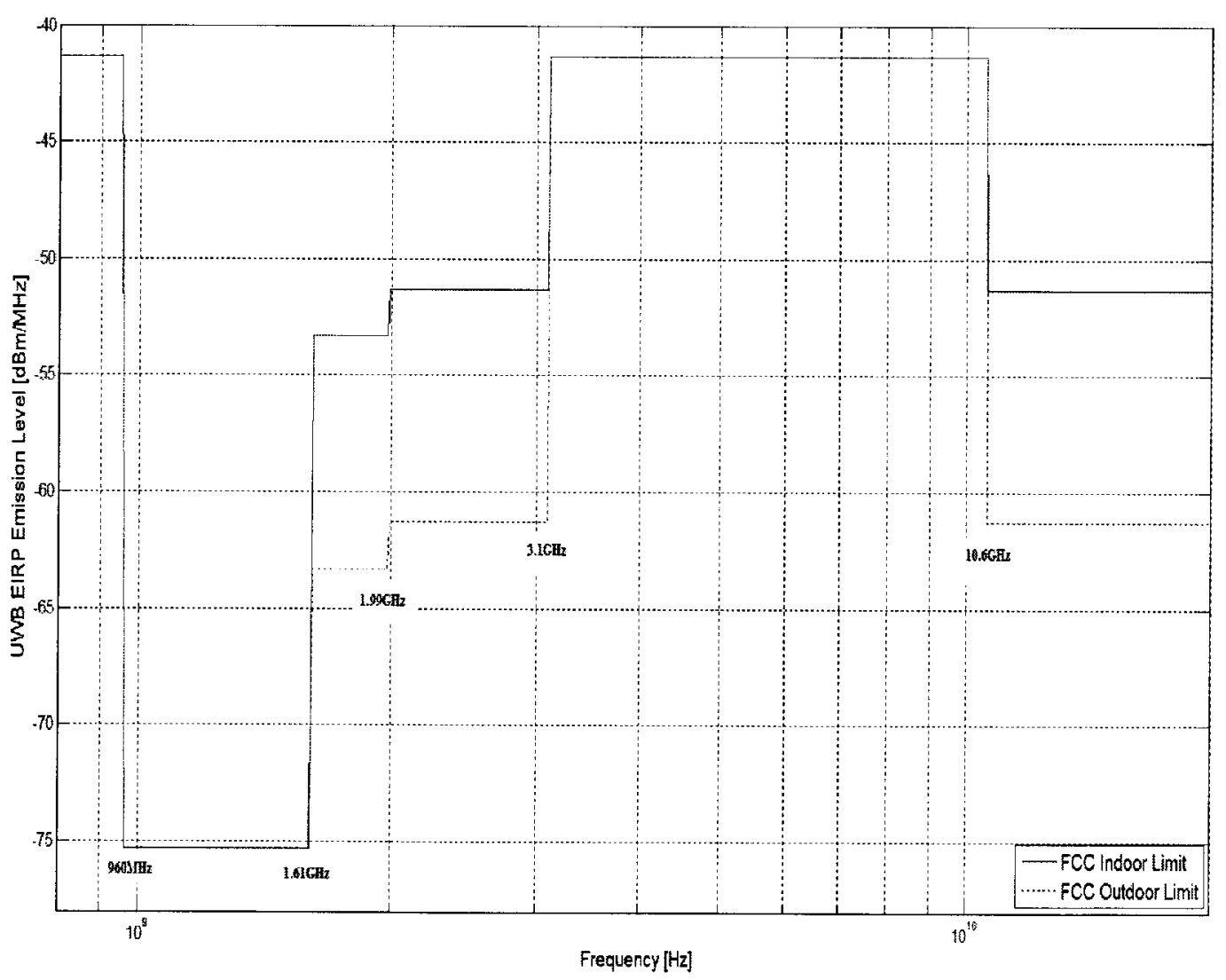

Figure 2.9: FCC UWB Indoor and Outdoor emission Masks [4]. 


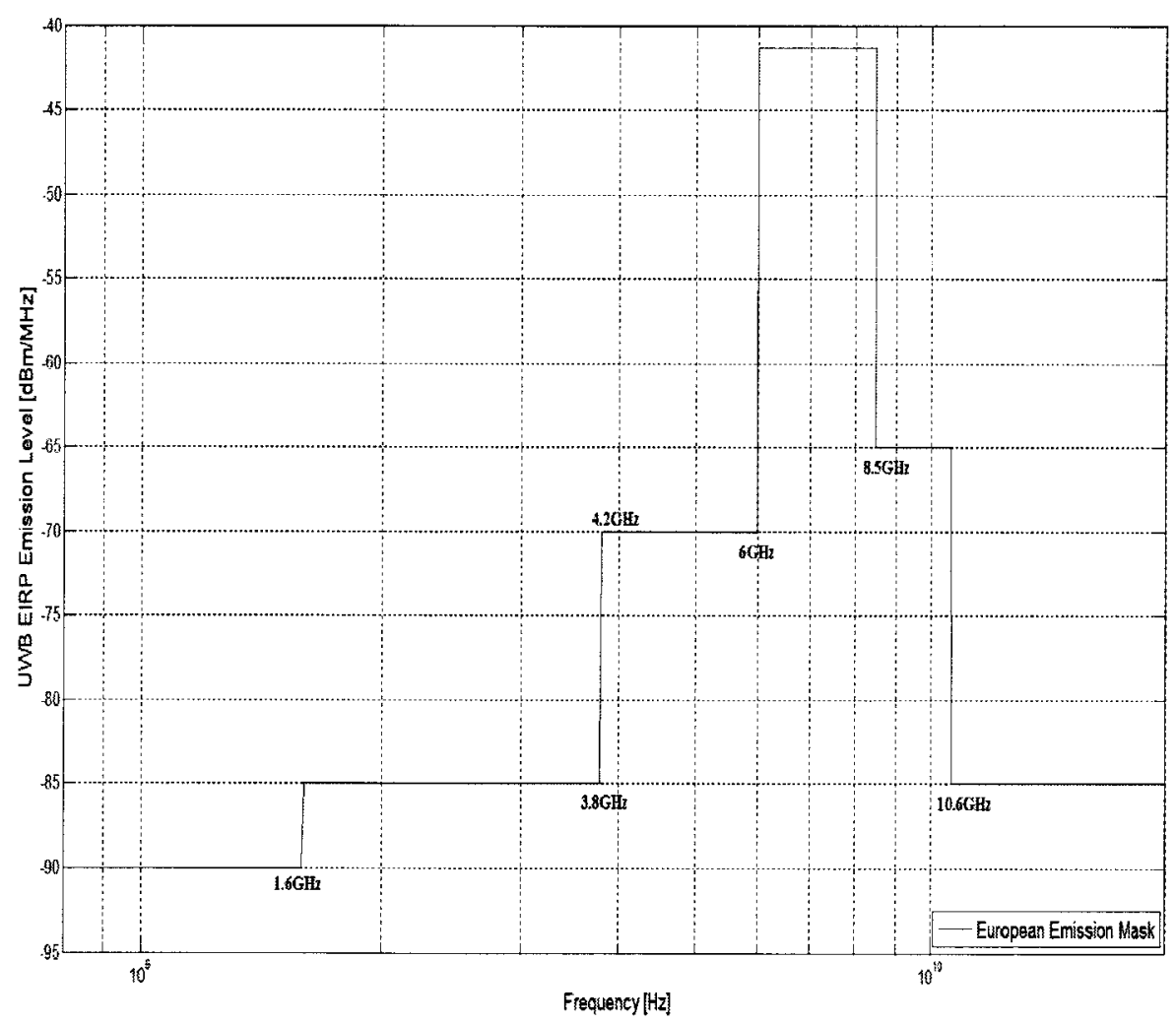

Figure 2.10: European UWB emission Mask [4]. 


\subsubsection{UWB regulations in Japan}

The Japanese UWB emission mask for indoor devices has two operating bands; from 3.4 to $4.8 \mathrm{GHz}$ and from 7.25 to $10.25 \mathrm{GHz}$. If the UWB transmitter uses detect and avoid mechanisms that monitor possible licensed devices in its vicinity, it is permitted to operate between 3.4 and $4.8 \mathrm{GHz}$ as shown in figure 2.11 [4]. It should be noted that operation between 7.25 and $10.25 \mathrm{GHz}$ is admissible also without the use of the detect and avoid mechanism.

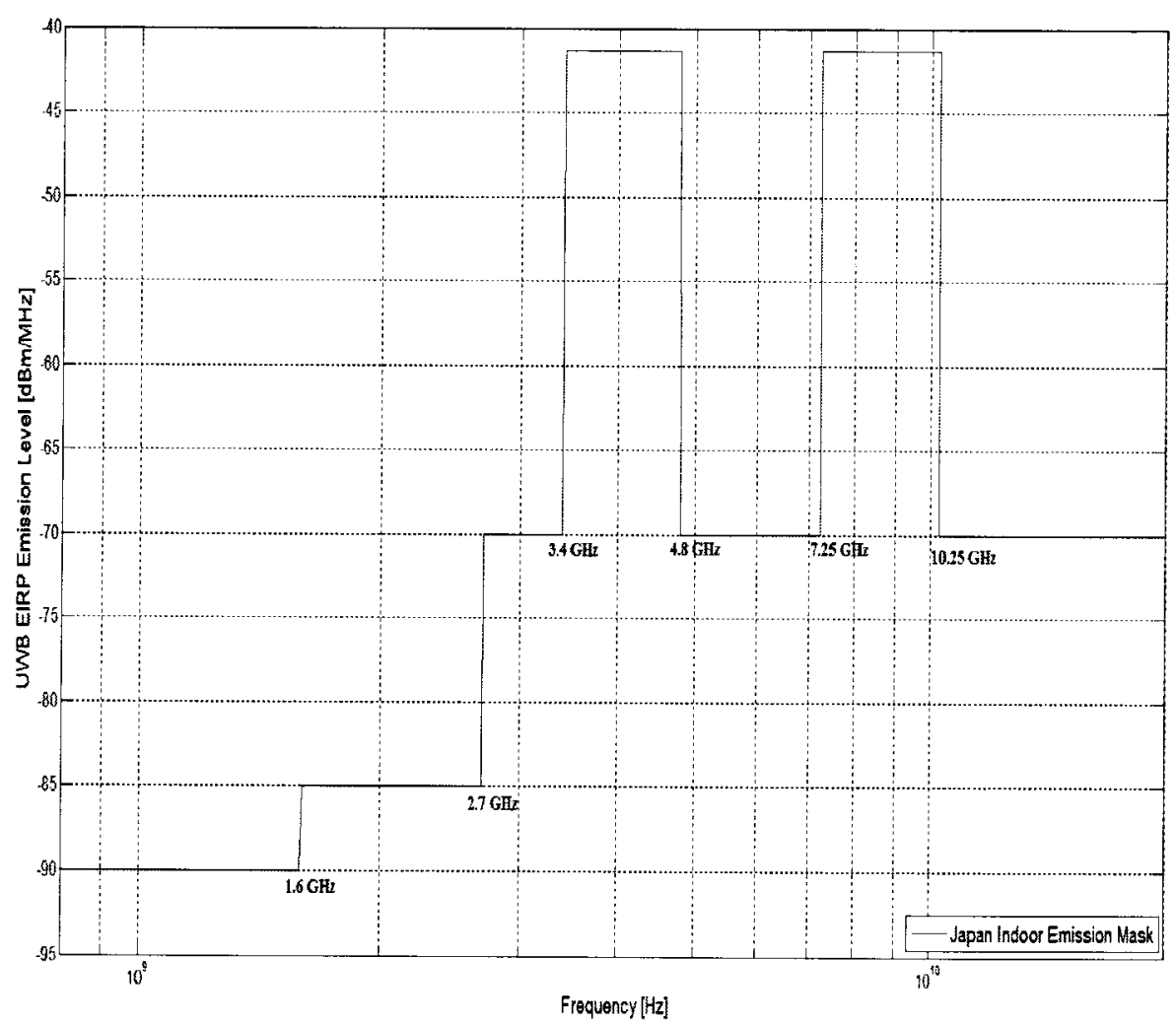

Figure 2.11: Japan UWB Indoor emission Mask [4]. 


\subsection{Types of Interference}

The desired UWB user is subjected to the effect of interference from other users or other operating wireless services. As a result, the performance of a UWB system is influenced by the effect of interference in a way much more than thermal noise. In the following, we are going to present the different kinds of interference that could influence the opcration of UWB systems.

\subsubsection{Multiple Access Interference}

Wireless communication systems encounter many problems to achieve a given quality of service (QoS), yet one of the most challenging problems is to accommodate as many users as possible in a finite amount of spectrum in accordance with the predetermined QoS. In order to solve such dilemma a multiple access scheme must be used to allow multiple users to share the same transmitting band in the same time.

The major multiple access techniques are: 1) frequency division multiple access (FDMA), where each user is assigned a different frequency slot. 2) time division multiple access (TDMA), where each user is assigned a different time slot. 3) CDMA, a unique spreading pseudo random code is used to spread the user signal. In practice, CDMA can accommodate more users than the other two types even in theory it shows that all the three have the same capacity [34].

The MAI is caused by the simultaneous presence of multiple UWB user signals. The MAI is modeled as the sum of other UWB user signals transmitting through the channel. These interfering signals are assumed to have the same basic characteristics as the desired user but with different signature.

In [35], an analytical method for statistical modeling of MAI, in TH-PPM, THPAM and DS-PAM UWB systems operating in AWGN channels had been introduced. Based on this method, BER formulas had been evaluated. General expressions for the BER performance in Log-normal fading multi-path channels had been also reported.

In [36], an accurate mathematical model explaining important features of the probability density function (PDF) of the MAI in TH-UWB systems was presented. The performances of several non-linear UWB receivers were also compared to each other and to the optimal benchmark ${ }^{8}$. It was found that the p-order metric receiver

\footnotetext{
8 The optimal receiver is achieved using the maximum a posteriori rule.
} 
[37] and the myriad filter detector [38] outperform all the other receivers and their performances are near the optimal performance.

\subsubsection{Inter Symbol Interference}

It is well known that at high data rates, some portion of the transmitted symbol energy unavoidably leaks into the following symbols, a fact which is known as intersymbol interference (ISI). ISI is one of the major factors degrading the detection performance of UWB systems. Therefore, for a successful implementation of high data rate UWB communications, it is compulsory to suppress ISI [39].

A filter is usually used in the receiver to compensate for the effect of distortion caused by both the channel and the transmitter. This filter is known as an "equalizing filter".

In [40] and [41], several Rake receivers including the all-Rake (ARake) receiver and selective-Rake (SRake) receiver were proposed for UWB reception and numerical results were provided to show the differences among these Rake receivers. It was shown in [42] that the SRake receiver can achieve better performance than the ARake receiver when ISI exists in the system and the DS-BPSK has the least susceptibility for ISI when compared to TH-PPM scheme. Further, the sensitivity of the performances of the Rake receivers to the number of Rake fingers is examined and the results show that 25-finger Rake receivers are required for good reception.

In [13], the bit error rate performance of the Rake reception of binary signals over the IEEE 802.15.3a UWB channel model is evaluated in the presence of ISI, inter-path interference, and inter-chip interference. A more accurate method is introduced by using the characteristic function of the interference and the AWGN instead of using Gaussian approximation for the PDF of the total disturbance.

In [14] a Rake receiver with recursive least squares equalizer for TH-UWB systems is proposed to mitigate the impact of ISI. Based on IEEE UWB channel models, it was shown that the proposed receiver scheme can eliminate the ISI effectively and improve the performance of the TH-UWB system under ISI.

\subsubsection{Narrowband Interference}

UWB signal is characterized by its huge bandwidth; this means that the UWB signal shares this bandwidth with many narrowband signals. Narrowband signals may 
exhibit a high PSD levels compared to the PSD of UWB signals as seen by a UWB receiver. Thus, many near band and in-band narrowband signals could degrade the performance of a UWB communication system.

In [27] and [43] the coexistence between the UWB system along with these narrowband operating services had been investigated, where the impact of interference from and to UWB system have been addressed. As presented in [44] and [45], the BER performance of the UWB receivers is greatly degraded due to the impact of NBI signals. The high processing gain of the UWB signal can only suppress a given amount of interference; if the level of interference becomes too great, the system will not function properly.

It can be seen that there exist many of the operating narrowband signals near the UWB bandwidth that may effect the UWB system operation such as GPS, where the L1-band and L2-band are operating in $1.575 \mathrm{GHz}$ and $1.227 \mathrm{GHz}$ respectively [46], universal mobile telephone service (UMTS) is another narrowband signal that uses frequency-division duplex (FDD) as a duplexing concept, the FDD uplink and downlink bands are between $1.92 \ldots 1.98 \mathrm{GHz}$ and $2.11 \ldots 2.17 \mathrm{GHz}$ respectively [47], wideband code division multiple access where it uses time-division duplex (TDD) as a duplexing concept, and the two separate TDD bands are located between $1.9 \ldots 1.92$ $\mathrm{GHz}$ and $2.01 \ldots 2.025 \mathrm{GHz}$ [48]. Finally, the IEEE802.11b WLAN that operates in the $2.4 \mathrm{GHz}$ band and uses the Direct-sequence spread spectrum modulation technique.

One of the main threats that operates within the UWB bandwidth is the presence of IEEE802.11a WLAN systems as they affect the performance of UWB systems [49]. The IEEE801.11a standard is based on the OFDM modulation scheme having a bandwidth of approximately $20 \mathrm{MHz}$. The modulation scheme ranges from BPSK up to 64-QAM. Together with a variable error-coding rate, OFDM modulation allows the data rate to be adapted from $6 \mathrm{Mbit} / \mathrm{s}$ to $54 \mathrm{Mbit} / \mathrm{s}$, depending on the propagation channel conditions.

Figure 2.12 depicts the operating narrowband interferer threats over the FCC UWB spectral mask for communication systems. 


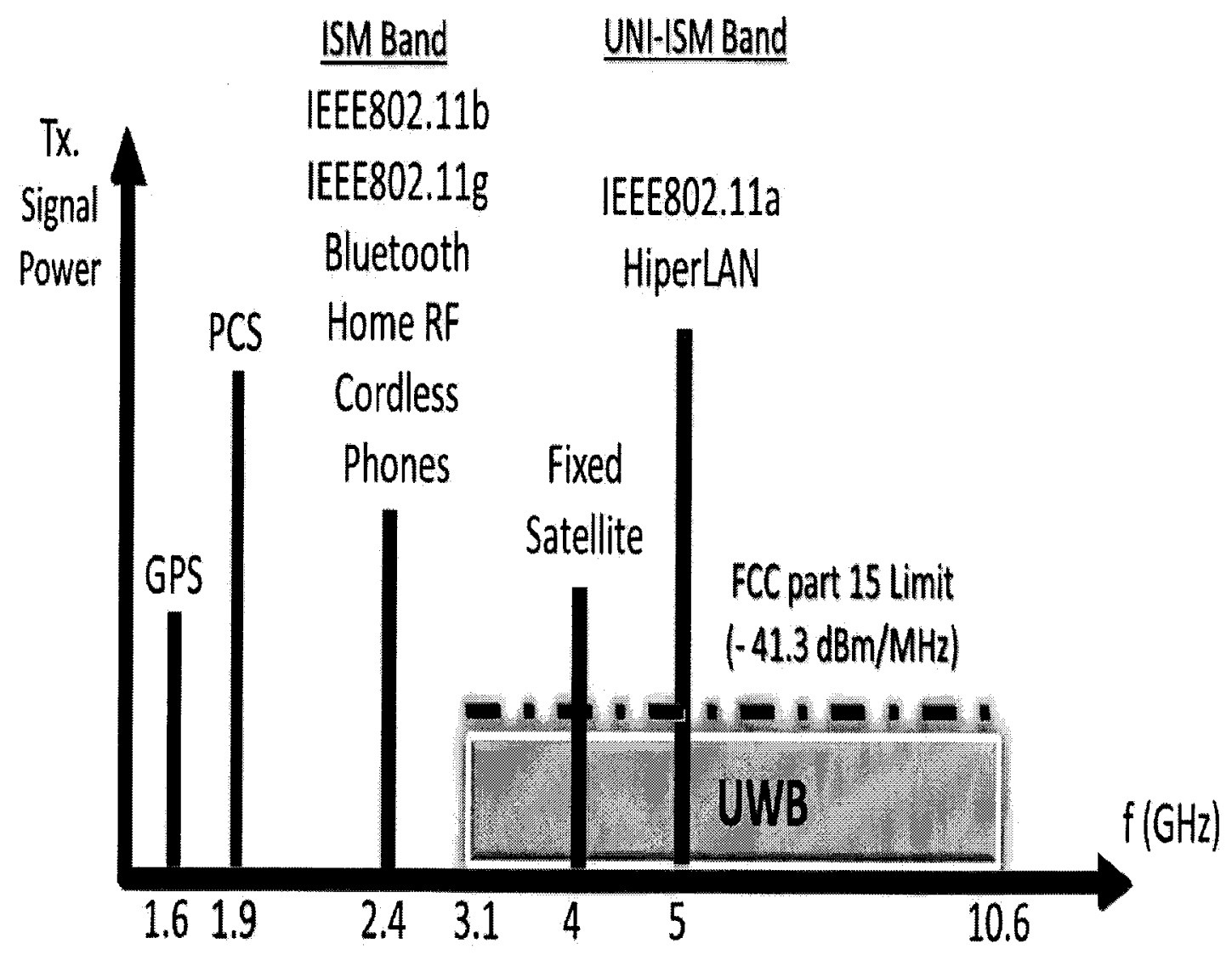

Figure 2.12: Narrowband interference threats over the FCC UWB spectral mask for communication systems [5]. 
The received UWB signal that includes all these types of interferences can be written as

$$
r(t)=S_{u w b}(t)+I_{N B I}(t)+I_{I S I}(t)+I_{M A I}(t)+n(t)
$$

where $\mathbf{n}(\mathrm{t})$ is the AWGN, $S_{u w b}(t)$ is the desired UWB signal, $I_{N B I}(t)$ is the interference from narrowband operating services, $I_{M A I}(t)$ is the interference due to the presence of UWB multiple access signals, and $I_{I S I}(t)$ is the interference resulting from ISI.

\subsection{Survey of NBI Suppression Techniques}

In this section, a survey of some of the already used interference cancellation techniques is presented. It had been stated that the presence of NBI signals may deteriorate the performance of IR-UWB communication systems. Therefore, in order to solve such problem; the UWB system could either employ NBI suppression techniques to improve its performance or could avoid transmission over the frequencies of strong narrowband interferers. These two methodologies are briefly presented in the following sub-sections and summarized at the end of the chapter.

\subsubsection{NBI Avoidance Techniques}

Effective techniques that are able to mitigate NBI need to be developed, along with the need of low complexity in both hardware and computation. Other limitations such as low-power and low-cost transceiver design in many UWB applications can also be considered. One approach to deal with NBI is to avoid the transmission of the UWB signal over the frequencies of possible strong narrowband interferers.

By properly designing the transmitted UWB waveform, NBI can be avoided at the UWB receiver. The UWB transmitter can adjust its transmission parameters appropriately if the statistics of the NBI are known. In the following sub-sections, we are going to presents some techniques that are used to avoid strong NBI signals.

\subsubsection{Pulse Shaping Technique}

Pulse shaping technique provides a means for the avoidance of NBI signals. For a binary pulse position modulated TH-UWB signal, the transmitted signal was presented in equation (2.12). The NBI is often modeled as a single tone interferer, which can 
be written as

$$
i(t)=\alpha \sqrt{2 I_{i}} \cos \left(2 \pi f_{c} t+\phi_{i}\right)
$$

where $\alpha$ is the channel gain coefficient, $I_{i}$ is the average power, $f_{c}$ is the center frequency of the sinusoid, and $\phi_{i}$ is the phase.

Since the coherence bandwidth of the channel is much larger than the bandwidth of the narrowband signal, the time domain samples of the NBI are highly correlated with each other. Therefore, the correlation function is of primary interest for the analysis of the narrowband signals and it can be written for a single tone interferer as

$$
R_{i}(\tau)=I_{i} \alpha^{2} \cos \left(2 \pi f_{c} \tau\right)
$$

The resulting correlation matrix for the $k^{\text {th }}$ and $\ell^{\text {th }}$ interference samples are [50]

$$
\left[R_{i}\right]_{k, \ell}=4 N_{s} I_{i}|\alpha|^{2}\left|P_{r}\left(f_{c}\right)\right|^{2}\left[\sin \left(\pi f_{c} \delta\right)\right]^{2} \cos \left[2 \pi f_{c}\left(\tau_{k}-\tau_{\ell}\right)\right]
$$

where $N_{s}$ is the number of pulses per information bit, $\left|P_{r}\left(f_{c}\right)\right|^{2}$ is the power spectral density of the received signal at the center frequency $f_{c}, \delta$ is the PPM modulation index, $\tau_{k}$ and $\tau_{\ell}$ are the propagation delays for the $k^{t h}$ and $\ell^{t h}$ path, respectively.

It is clear from the previous equations that the effect of interference is directly related to the spectral characteristics of the receiver template pulse waveform. That means, if the transmission at the frequencies where NBI is present can be avoided, the influence of interference on the received signal can be mitigated significantly. Therefore, by designing the transmitted pulse shape properly, such that the transmission at some specific frequencies is omitted, NBI avoidance can be achieved.

One of the excellent examples for the implementation of this approach is the Gaussian doublet [51]. A Gaussian doublet consists of a pair of narrow Gaussian pulses with opposite polarities for representing one bit. The power spectral density of the doublet exhibits nulls at certain frequencies related to the time delay between the doublet two pulses.

Another approach of NBI avoidance with the aid of pulse shaping has been suggested in [52], where they introduced a "coded Gaussian mono-cycle pulse" in which Gaussian mono-cycles are weighted, delayed and summed in accordance with a designed codeword. The designed Gaussian mono-cycles generate a spectral null at 
the frequencies being used by existing narrowband interferers.

Several research studies have addressed the pulse shaping technique in order to mitigate the NBI impact on the UWB system. For example, in [53] and [54] UWB pulse design using PSWF, which can notch the NBI signal, are proposed but the pulse is quite difficult to be generated.

\subsubsection{Multi-carrier Approach}

A well-known example of this approach is the OFDM technique, where narrowband interferes affect some carriers in the OFDM spectrum which in turn affect the information that is transmitted over these frequencies. If the interfered carriers can be identified, transmission over these carriers can be avoided.

As presented in [55] and [56], mitigating the impact of NBI on the performance of a pulse based UWB receiver is achieved by means of a multi-carrier template wave. The NBI signal is modeled as a multi-band frequency hopping pattern. Detection to the interference band is initially done, and then the NBI mitigation is achieved, where the interfered sub-carriers can be eliminated.

In such cases, the receiver should have the ability to identify the interfered carriers and to report them to the transmitter that in return adjusts its transmission accordingly.

In order to achieve this, the interference characteristics need to be constant for a certain period of time. As if it changed very fast, the transmitter will wrongly adjust its parameters.

Another drawback of this approach is when the number of NBI signals increases. Then, the number of interfered carriers that need to be avoided will be increased as well, which will reduce the system's throughput.

\subsubsection{Multi-band Approach}

Multi-band approach can be another way of avoiding NBI signals. The idea of the multi-band technique is based on dividing the UWB spectrum into smaller sub-bands instead of using the whole $7.5 \mathrm{GHz}$ UWB bandwidth.

Optimization of the system performance can be achieved by the suitable combination of these sub-bands. In such cases information on each of the sub-bands 
can be transmitted using either single-carrier (pulse-based) or multi-carrier OFDM techniques.

Figure 2.13 shows some of the proposed multi-band schemes. The pulse-based approach uses dual-band, the lower band occupies the spectrum from $3.1 \mathrm{GHz}$ to 4.85 $\mathrm{GHz}$, and the upper band occupies the spectrum from $6.2 \mathrm{GHz}$ to $9.7 \mathrm{GHz}$. Due to the potential existence of the interference sources IEEE802.11a, which operates at the $5 \mathrm{GHz}$ frequency band, the spectrum in between the upper and the lower bands is not used for UWB transmission. In case of the OFDM-based multi-band approach the three lower band channels are mandatory and set for initial deployments. The upper bands are optional and for future use, where they are expected to be included in the system gradually by the improvement of the radio frequency technology.

\subsubsection{Physical NBI Avoidance Techniques}

In addition to the previously mentioned approaches, physical solutions can also be considered for avoiding NBI, such as using antenna design. By intentionally adding a narrowband resonant structure to the antenna, frequency notches will be created [57] and [58]. In [8], two antennas are presented, where one antenna is designed for single band-notch with a separated strip to cover the 5.15-5.825 GHz band. The second antenna is designed for dual band-notches using two separated strips to cover the 5.15-5.35 GHz band and 5.725-5.825 GHz band. Figure 2.14 depicts configurations of both the single and dual band notches UWB antenna.

This technique is more economical than the explicit notch filtering method since it does not require additional notch filters. It is necessary to note that the performance of the antenna is reduced with increasing number of notches. Thus, we can conclude that the frequency notched antenna does not perform well in the presence of numerous simultaneously existing narrowband interferers.

\subsubsection{NBI Cancellation Techniques}

The main limitation on the previously mentioned NBI avoidance approaches is their dependency on the exact knowledge about narrowband interferers. Accurate information about the center frequency of the interference is necessary to be effective for the NBI avoidance techniques. Also, in the presence of large number of narrowband interferers, these avoidance approaches become impractical. In the following, an overview 


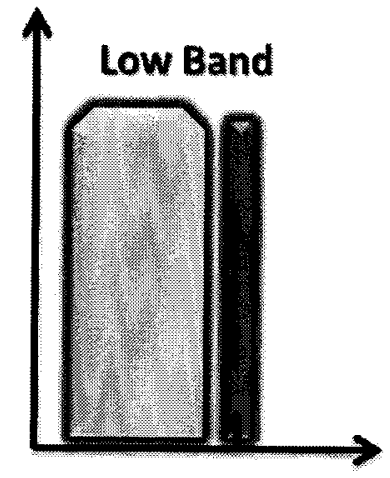

$5 \mathrm{GHz}$
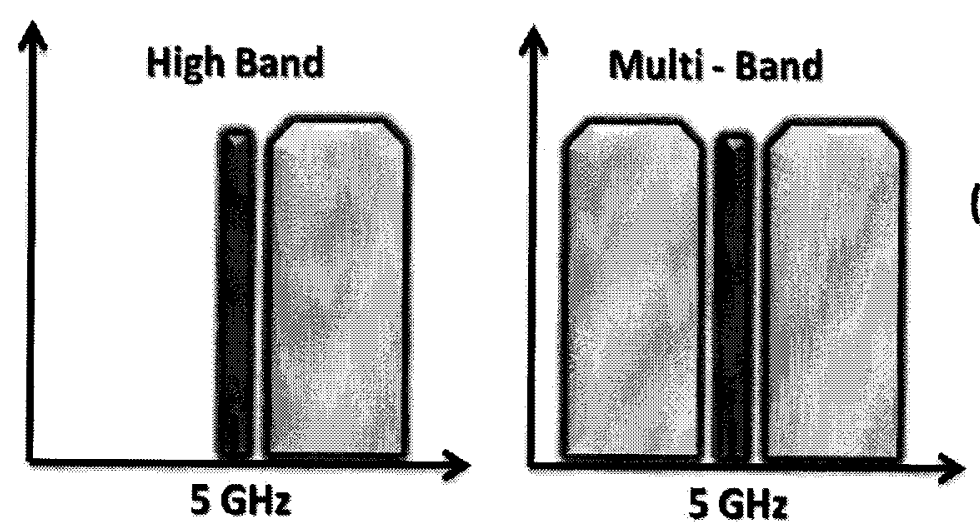

(a)

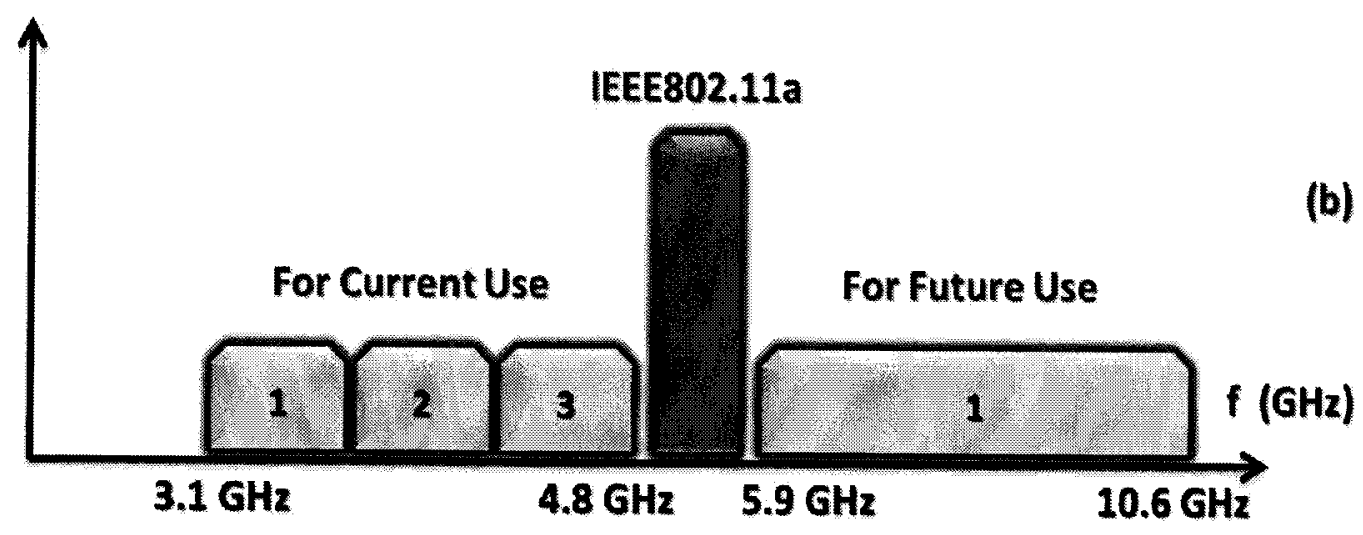

Figure 2.13: (a):Dual-band approach as proposed by the Xtreme SpectrumMotorola [6], (b): The multi-band OFDM [7] 

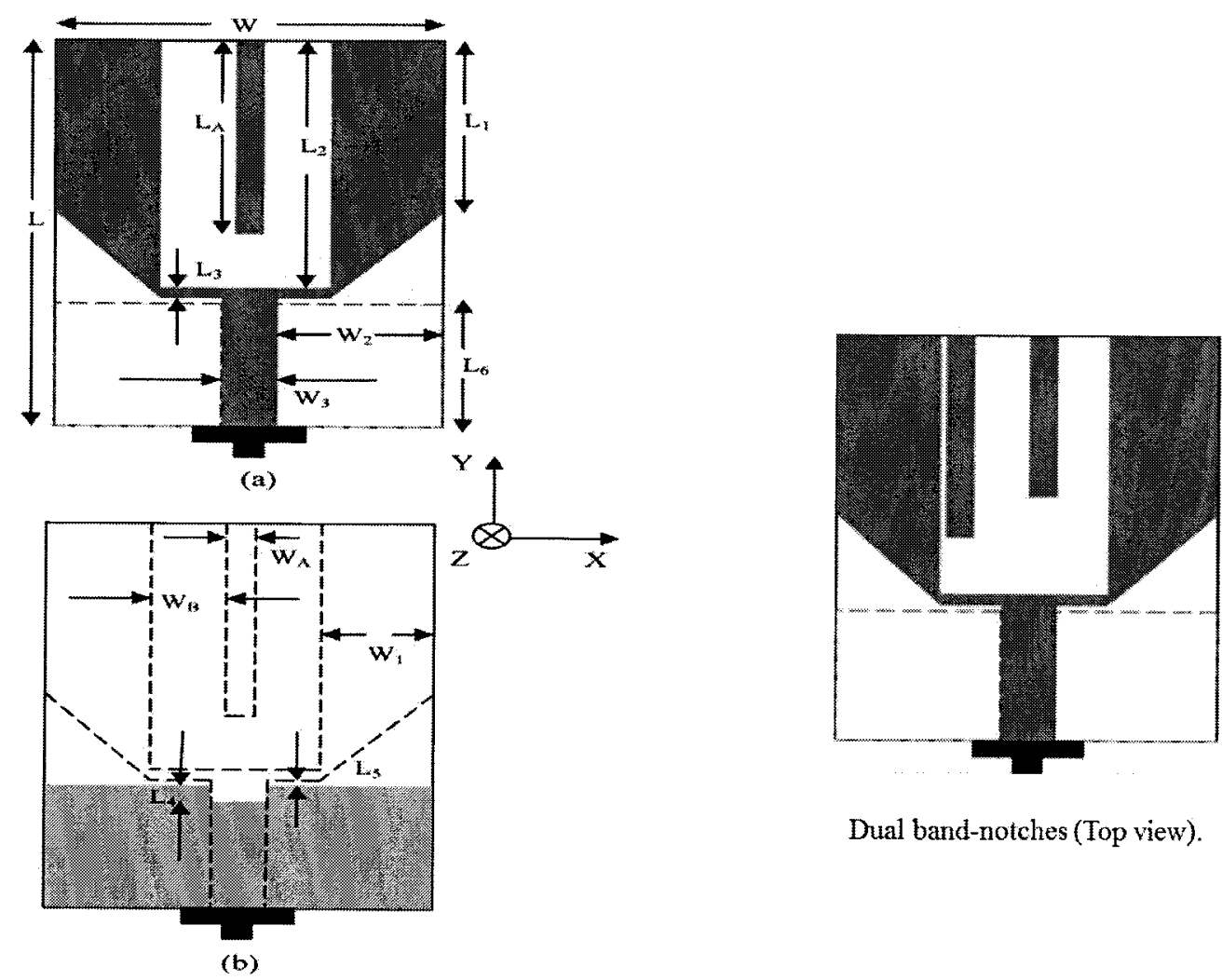

Dual band-notches (Top view).

Single band-notch (a- top view and b- bottom view).

Figure 2.14: Configuration of the single and dual band notches UWB antenna [8] 
of the second category of NBI mitigation techniques known as "NBI Cancellation" is presented.

\subsubsection{Frequency Domain Techniques}

These techniques relay largely on a notch filter in the receiver side. Notch filters can be used to suppress NBI if an accurate estimation about the frequencies of the powerful narrowband interferers is accomplished [59].

As presented in [60], to combat interference in multi-band OFDM UWB systems, an analog notch filter is designed to be included after down-conversion and low-pass filtering in the UWB receive chain. The filter utilizes digital center frequency control, and an analog least-mean-square control for minimizing the interference amplitude. The center frequency was measured to be tunable from 1.6 to $278 \mathrm{MHz}$, and the bandwidth was maintained around $23 \mathrm{MHz}$ throughout the tuning range.

However, the main drawback of the frequency domain approach lies on the fact that they are useful only when the received signal ${ }^{9}$, exhibits stationary behavior. However, in the scenario of a time-varying received signal, methods known as the "time - frequency" approaches that analyze the frequency content taking the temporal changes into account are required.

\subsubsection{Time - Frequency Domain Techniques}

The wavelet transform is the most commonly employed time-frequency domain technique for interference suppression. The wavelet transform is based on the use of basis functions, and the express of any time domain signal as a combination of these functions. However, these basis functions (wavelets) are time limited ${ }^{10}$. Hence, the wavelet transform is able to represent the time local characteristics of signals.

As suggested in [61] by representing the UWB transmission waveform as wavelet packet coefficients and by selectively removing those coefficients whose spectral footprint lie in or close to the interference zones, the effect of interference on the UWB system is mitigated.

Obviously, this method is quite similar to the multi-carrier approach in NBI avoidance techniques. Another method employing wavelet transform is presented in [62]

\footnotetext{
${ }^{9}$ The superposition of the UWB signal and NBI from various sources.

${ }^{10}$ These wavelets are different from the complex exponentials used by the Fourier transform.
} 
and [63]. This time the processing is done in the receiver side, by applying notch filtering on the interfered frequencies.

Although the discrete wavelet transform can be considered a very useful tool for eliminating NBI, the inability of current analog to digital converters to sample the UWB signals at the Nyquist rate sets a practical limit on the feasibility of this method.

\subsubsection{Time Domain Techniques}

As presented in [64], the interference can be considered as a narrow-band process, so its future values (and, in particular, its current value) can be predicted from past values. Hence, the current value, once predicted, can be subtracted from the incoming signal, leaving a waveform comprised primarily of the wideband signal and the thermal noise.

Time domain techniques can be classified into linear and nonlinear techniques. The linear techniques employ transversal filters in order to obtain an estimate of the received signal depending on the model assumptions and previous samples [65]. In [66] a NBI cancellation unit has been presented, where it estimates and regenerates the interference then eliminates it by subtracting to a delay version of the received signal. The estimation of the interference is achieved with the aid of a least mean squares (LMS) algorithm along with a multiple tap LMS filter.

Whereas, the non-linear techniques, such as the "adaptive prediction filters" [67], in which a notch to the interference frequency is created by the adaptation algorithm once the interference is detected. However, the drawback of this technique appears if the source of interference vanishes suddenly, the receiver continues notching the desired signal.

\subsubsection{Minimum Mean Square Error Combining}

The well-known Rake receiver is mostly used to intercept UWB signals with the aid of maximal ratio combiner (MRC) as a combining technique. However, in the presence of NBI, and since the NBI samples are correlated, MRC is no longer the optimum method to be used. Alternatively, Minimum Mean Square Error (MMSE) combining techniques can be used and it depends on varying the weights of the Rake fingers in such a way that the mean square error between the required and actual outputs is minimized [68]. However, by using the MMSE technique the computational complexity will be increased dramatically. 


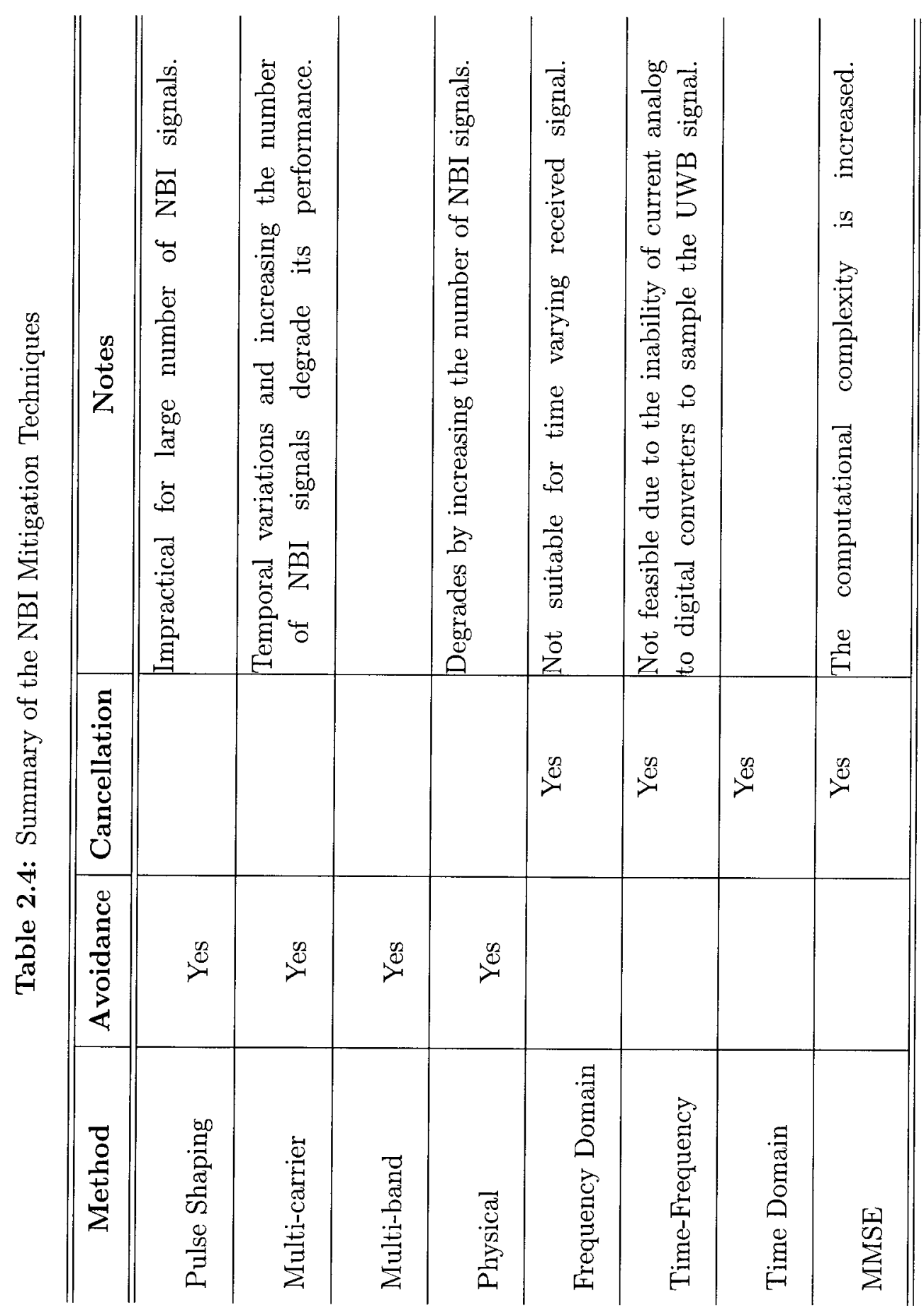




\section{Chapter 3}

\section{The Interference Canceller Scheme (ICS)}

\subsection{Introduction}

In this chapter, we propose a novel cancellation technique to mitigate the impact of the narrow band interfering signals on IR-UWB receiver system. The ICS attempts to subtract the interfering signal from the incoming received signal. Assuming that the UWB receiver is subjected to a single high power NBI signal, the interference canceller operates by: 1) the incoming received signal will be split into two paths. In the first path (the NBI path) an attempt to demodulate the NBI signal from the incoming received signal is done. 2) The output from the demodulation process will pass through the second stage that regenerates the NBI signal. 3) The regenerated NBI signal will be multiplied by the NBI channel estimate prior to its subtraction from the incoming received signal in the second path (the incoming received signal path) at the input of the UWB receiver.

If the demodulation process is successful and the NBI channel model is perfectly known, the NBI signal can be perfectly suppressed from the incoming received signal and the reception of the UWB signal can be achieved as if there was no interference.

Figure 3.1 depicts a simplified schematic model of the proposed ICS. The performance of such canceller scheme is addressed thoroughly taking into consideration the UWB channel models, multiple access interference and symbol timing errors. The case of multiple NBI is also considered. The ICS performance is compared to the performance enhancement based on the use of a notch filter.

Note that, the UWB signal at the input of the NBI receiver can be considered as 


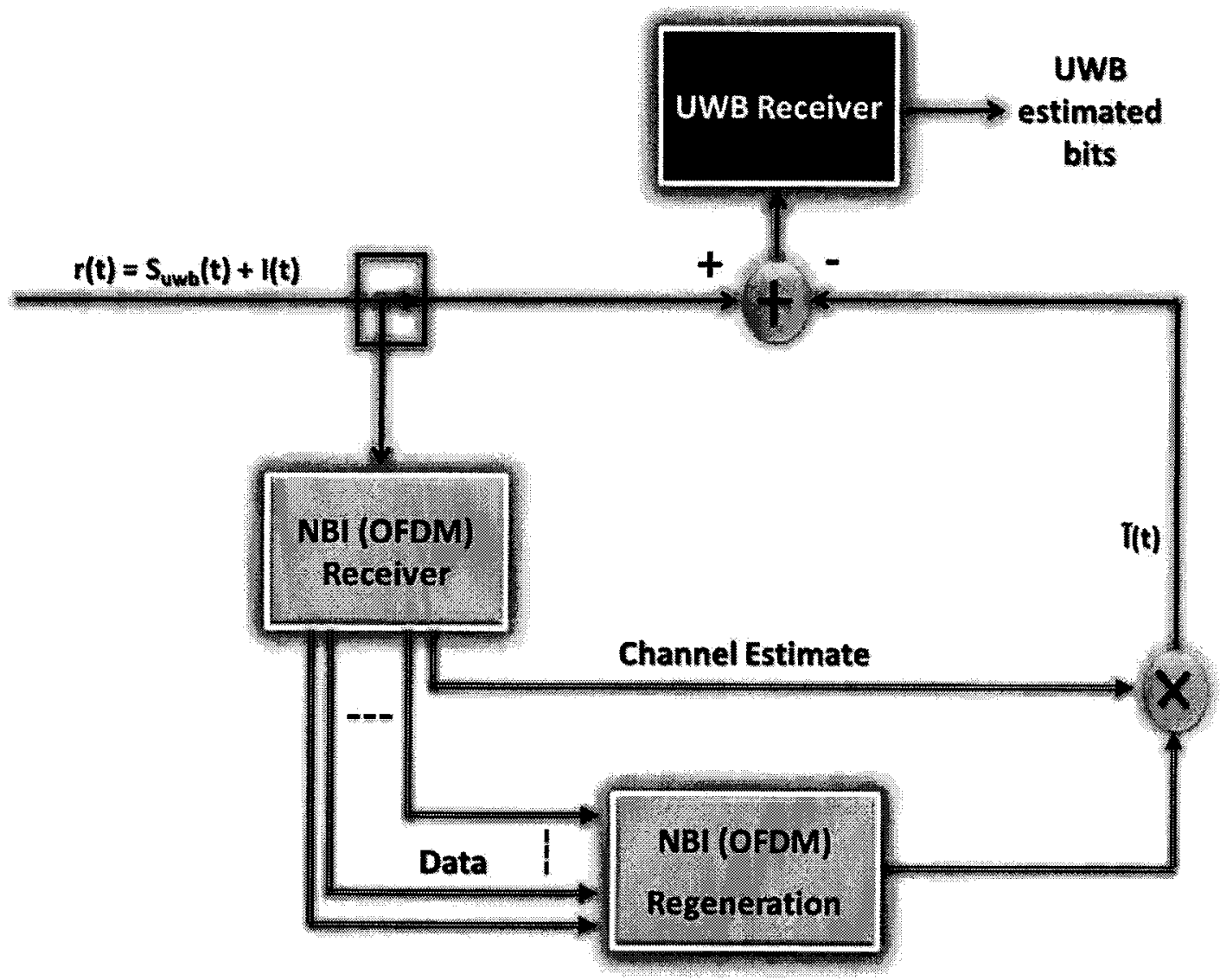

Figure 3.1: A general block diagram of the ICS. 
an interfering signal ${ }^{1}$ and its influence on the ability of the narrowband receiver to demodulate efficiently the NBI signal must be investigated.

\subsection{The Impact of UWB Interference on Narrow- band Receiver}

Before we analyze the performance of such canceller scheme, we initially investigate the impact of UWB signal on the performance of a narrowband receiver. The narrowband signal is modeled as the standard OFDM based IEEE802.11a WLAN. The UWB signal can be considered as an additional interfering noise signal along with the AWGN at the input of the narrowband receiver. This additional noise may affect the performance of the narrowband receiver ${ }^{2}$.

The signal to noise plus interference ratio (SNIR) at the input of the OFDM based NBI receiver ${ }^{3}$ can be written as

$$
(S N I R)_{O F D M}^{U W B-A W G N}=\left(\frac{S_{o f d m}}{\left(N_{o}+\frac{S_{u w b}}{B_{u w b}}\right) B_{o f d m}}\right)
$$

where $B_{o f d m}$ is the OFDM narrowband signal bandwidth and $B_{u w b}$ is the UWB bandwidth $\approx 1 / T_{m}$, where $T_{m}$ is the UWB pulse duration.

Equation (3.1) can be further rearranged as:

$$
(S N I R)_{O F D M}^{U W B-A W G N}=\left[\left(\frac{E_{b}}{N_{o}}\right)_{O F D M-A W G N}^{-1}+\left(\frac{(S I R)^{-1}}{\frac{B_{o f d m}}{B_{u w b}}}\right)^{-1}\right]^{-1}
$$

$S I R$ is the average signal to interference power ratio, defined as:

$S I R=\frac{S_{u w b}}{S_{o f d m}}$, where $S_{u w b}$ is the UWB signal power and $S_{o f d m}$ is the OFDM narrowband signal power.

The influence of the UWB interference on the probability of symbol error of a

\footnotetext{
${ }^{1}$ It can be considered as an additional AWGN, which can increase the noise floor of the NBI receiver.

${ }^{2}$ A deterioration in the performance of the narrowband receiver leads to a deterioration in the overall performance of the canceller scheme.

3 The NBI OFDM receiver is the first stage of the ICS.
} 
quadrature phase shift keying (QPSK) OFDM modulated system is depicted in

figure 3.2 for different signal to interference ratios $\left(S I R_{o}=S_{o f d m} / S_{u w b}\right)$ as seen by the OFDM narrowband receiver.

A SNR degradation is expected to reach up to $3 \mathrm{~dB}$ at $\mathrm{BER}=1 \times 10^{-3}$ for a $S I R_{o}=-10 \mathrm{~dB}$. Note that, when the $S I R_{o} \geq 0 \mathrm{~dB}$, the impact of UWB signal on the performance of the OFDM narrowband system can be neglected.

In order to validate our analytic results, we followed the same approach presented in [69]. The performance of OFDM narrowband receiver in the presence of UWB interference is evaluated and depicted in figure 3.3. The BER performance of the OFDM narrowband receiver in AWGN channel is evaluated assuming the standard Gaussian approximation (SGA) hypothesis for an Energy per Bit, $E_{b}$ to the noise spectral density, $N_{o}$ ratio, $E_{b} / N_{o}=10 \mathrm{~dB}$, and for different $S I R_{o}$ ratios.

We can conclude from these figures that the UWB interference signal could degrade the performance of the OFDM narrowband receiver, especially when the UWB interference power is greater than the OFDM narrowband signal power. However, the impact of the UWB interference signal decreases for large OFDM narrowband signal power and can be neglected.

\subsection{Performance of the ICS in AWGN Channel}

In this section, the performance of the ICS is evaluated in AWGN channel environment. Assuming that we operate under perfect channel estimation conditions ${ }^{4}$, the narrowband receiver probability of symbol error can be summarized into one of the following cases:

\section{Case 1: No Symbol Errors}

Assuming successfully demodulating the NBI signal, this means that the narrowband receiver produces no symbol errors. The demodulated bits/symbols will pass through the second stage, which is responsible for regenerating the NBI signal. As expected the NBI signal will be cancelled efficiently at the input of the UWB receiver.

The BER performance of a UWB system using the canceller scheme in this case can be considered equivalent to the BER performance of a UWB system operating in

\footnotetext{
${ }^{4}$ For both the desired UWB and NBI channel models.
} 


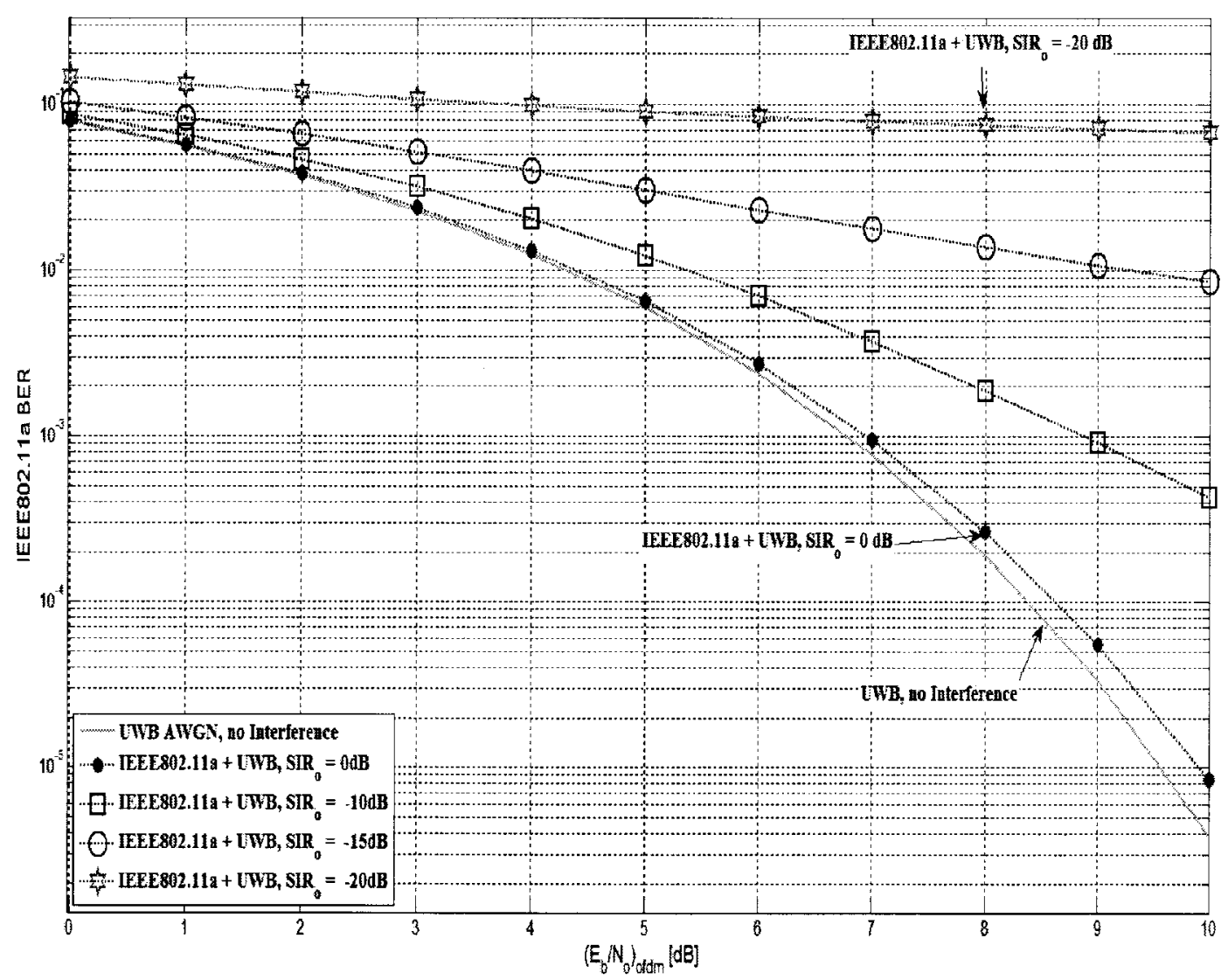

Figure 3.2: Analytic evaluation of the BER performance of a QPSK-OFDM narrowband system in AWGN channel in the presence of UWB interference signal and $B_{u w b} / B_{o f d m}=125$. 


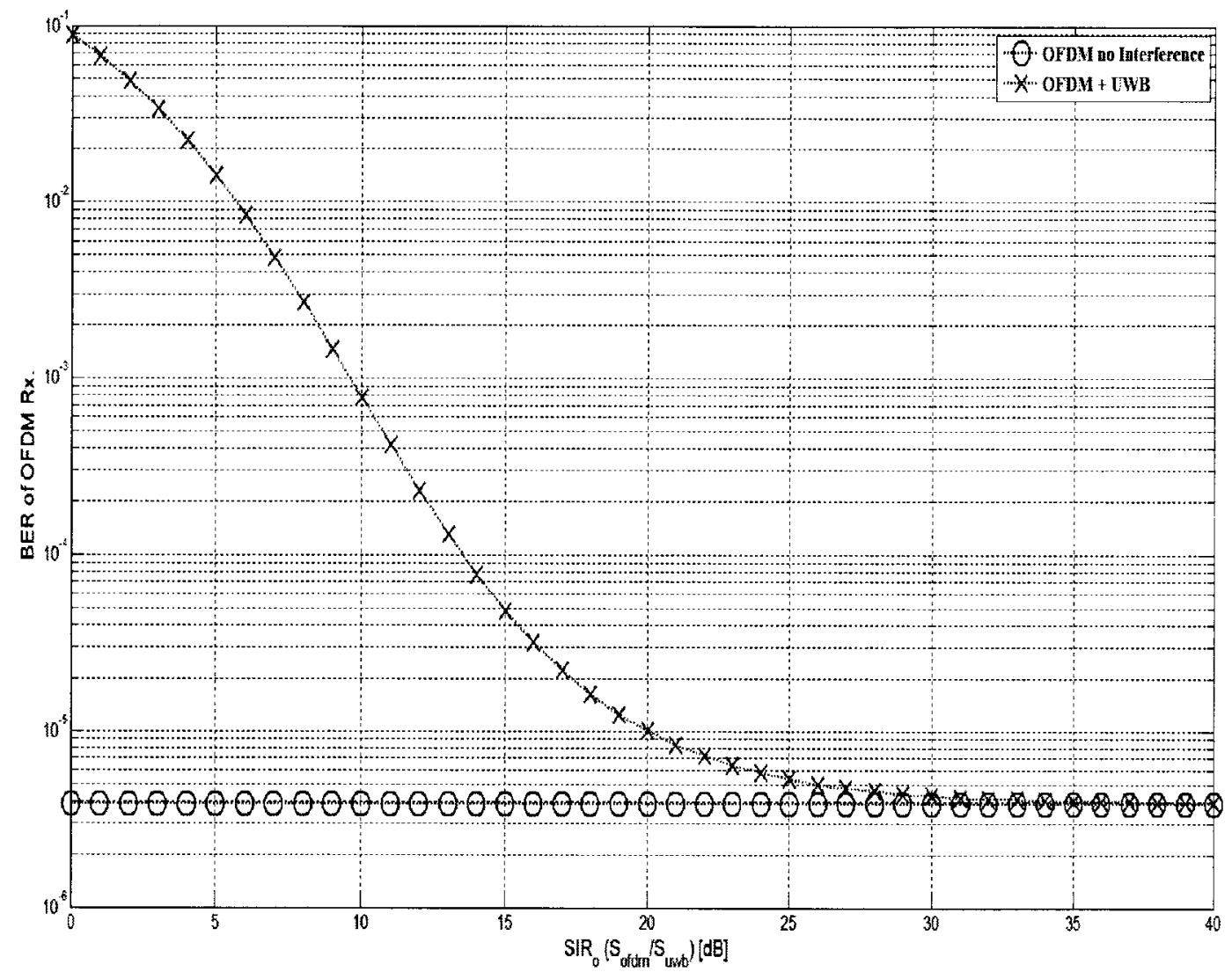

Figure 3.3: BER of QPSK-OFDM narrowband system in AWGN channel in presence of UWB interference signal, $E_{b} / N_{o}=10 \mathrm{~dB}$.

AWGN channel in absence of interference. It can be written as

$$
\left(P_{e}\right)_{u w b-A W G N}^{i c s}=Q\left[\sqrt{\frac{E_{b}}{N_{o}}(1-\varrho)}\right]
$$

where $\varrho$ is the correlation coefficient between the two UWB pulses, $p(t)$ and $p(t-\delta)$ for the two bits $(0,1)$ respectively. $\varrho$ can be defined as $[9]^{5}$

$$
\varrho=\int_{-\infty}^{\infty} p(t) p(t-\delta) d t
$$

The value of $\varrho$ ranges between $[-1,1]$, where $\varrho=-1$ corresponds to the antipodal modulation, $\varrho=0$ corresponds to the orthogonal modulation, and $\varrho \in(0,1]$, and $\varrho \in$

${ }^{5}$ Chapter 9, Page (375), equation (9-18). 
$[-1,0)$ corresponds to modulation schemes that are inferior and superior to orthogonal modulation.

\section{Case 2 : Multiple Symbol Errors}

The second case is where the narrowband receiver produces multiple symbol errors $^{6}$. The BER performance of the canceller scheme can be written as

$$
\begin{aligned}
& \left(P_{e}\right)_{u w b}^{i c s}=\left(P_{e-u w b}^{A W G N}\right)\left(1-P_{e-o f d m}^{e=1}-P_{e-o f d m}^{e=2}-\ldots-P_{e-o f d m}^{e=e^{\prime}}\right) \\
& \quad+\left(P_{e-u w b}^{1}\right)\left(P_{e-o f d m}^{e=1}\right)+\left(P_{e-u w b}^{2}\right)\left(P_{e-o f d m}^{e=2}\right)+\ldots+\left(P_{e-u w b}^{e^{\prime}}\right)\left(P_{e-o f d m}^{e=e^{\prime}}\right)
\end{aligned}
$$

where $\left(P_{e-u w b}^{2}\right)$ and $\left(P_{e-u w b}^{e^{\prime}}\right)$ are the probabilities of error of the UWB system in the presence of two and $e^{\prime}$ tone interferers respectively. $\left(P_{e-o f d m}^{e=2}\right)$ and $\left(P_{e-o f d m}^{e=e^{\prime}}\right)$ are the probabilities of occurring two and $e^{\prime}$ symbol errors in a block of length $\mathrm{N}=52$ respectively.

The probability that the NBI receiver produces "e" symbol errors over a block of "N" symbols is given by [70]

$$
P_{O F D M}^{e}=\frac{N !}{(N-e) ! \times e !} P^{e}(1-P)^{N-e}
$$

where "N" can be interpreted for an OFDM NBI signal as the number of the OFDM data sub-carriers, and "P", is the probability of symbol error.

For a QPSK OFDM-based NBI signal, the probability of symbol error is given by $[39]$

$$
P_{s e}^{O F D M}=2 Q\left[\sqrt{\frac{2 E_{b}}{N_{o}}}\right]-Q^{2}\left[\sqrt{\frac{2 E_{b}}{N_{o}}}\right]
$$

Figure (3.4) presents the probability that the NBI receiver produces " $\mathrm{e}=1$ " symbol error over a block of "N" symbols for different $\left(E_{b} / N_{o}\right)$ and at different SIRs $=0$, and $-20 \mathrm{~dB}$.

Figure 3.5 shows the probability of symbol errors $(e=1,5,10,15$ and 20$)$ in a

\footnotetext{
${ }^{6}$ Multiple symbol errors mean two or more symbol errors in a block of length $\mathrm{N}$.
} 


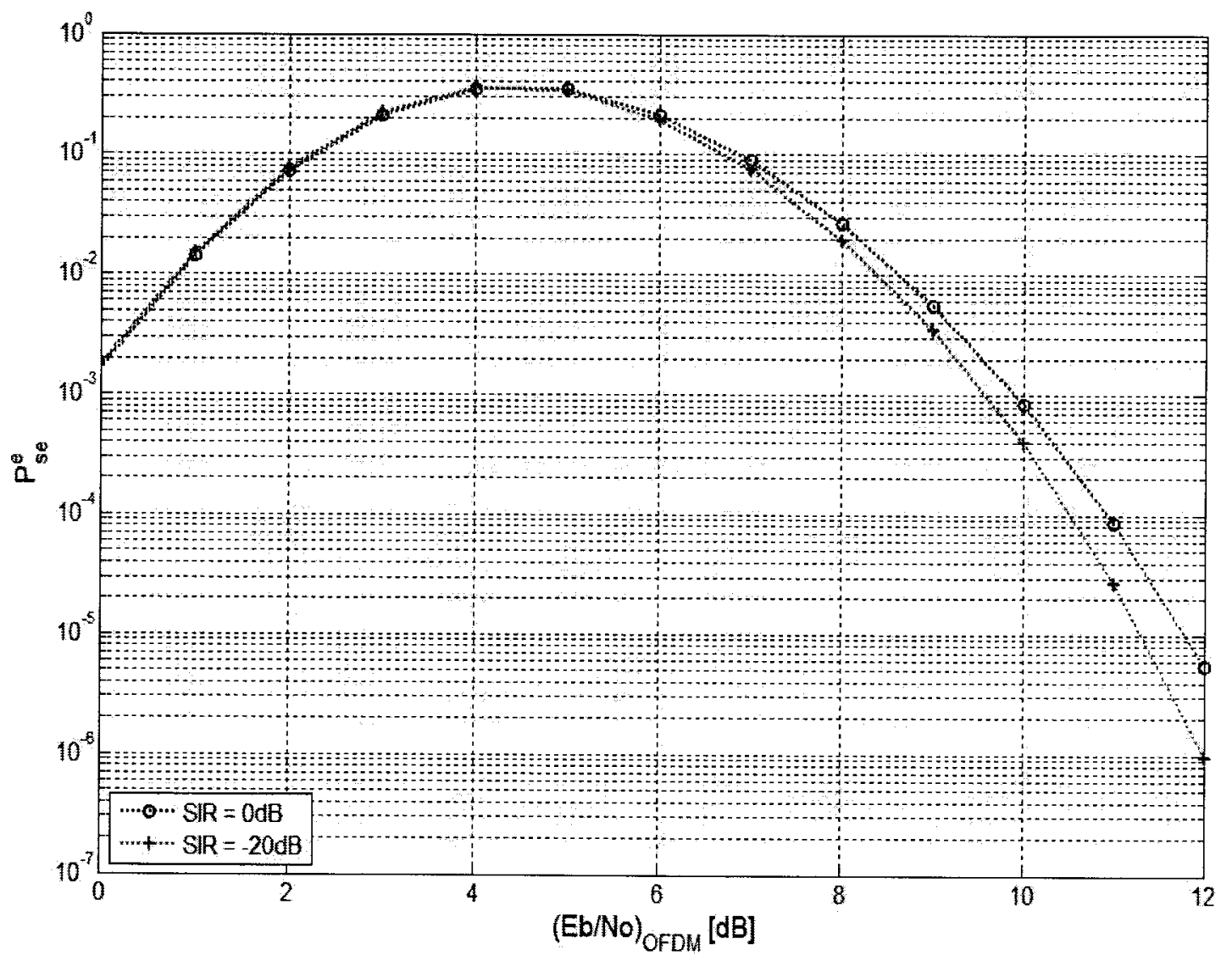

Figure 3.4: NBI receiver probability of symbol error with " $e=1$ " symbol errors over a block $\mathrm{N}=52$ symbols at different $\mathrm{SIR}=0$, and $-20 \mathrm{~dB}$. 
block of length $\mathrm{N}=52$ at a $\mathrm{SIR}=0 \mathrm{~dB}$.

We can conclude from this figure that the probability of multiple symbol errors for the OFDM narrowband receiver is highly dependent on "e"; for e $>15$, this probability is negligible.

\subsection{IR-UWB Performance in the Presence of NBI in AWGN Channel}

The performance of IR-UWB communication systems in the presence of $N_{i}$ tone interferers has been evaluated in AWGN channel in [71]. In particular, they derived closed-form bit-error probability expressions for UWB systems in the presence of NBI signal in various UWB channel models, by approximating narrowband interferers as independent asynchronous tone interferers. In the case of TH-PPM, the probability of error is given by [71]:

$$
\begin{array}{r}
P_{e}=Q\left(\sqrt{\frac{E_{b}}{N_{o}} \cdot(1-\varrho)}\right)+1 / \pi \int_{0}^{\infty}\left[1-\prod_{n=1}^{N_{i}} J_{o}\left(\omega \sqrt{\frac{I_{n}}{C} \cdot \frac{\left|M\left(f_{n}\right)\right|^{2}}{T_{b}} \cdot \frac{2}{(1-\varrho)^{2}}}\right)\right] \\
\cdot \frac{\sin (\omega)}{\omega} \cdot \exp \left(-\frac{\omega^{2}}{2} \cdot \frac{N_{o}}{E_{b}} \cdot \frac{1}{(1-\varrho)}\right) d \omega
\end{array}
$$

where $C=E_{b} / T_{b}$ is the desired received power, $J_{o}(\omega)$ is the $0^{\text {th }}$ order Bessel function of the first kind and $I_{n} / C$ is the average SIR corresponding to the $n^{\text {th }}$ interferer at frequency $f_{n}$.

$|M(f)|$ is the transfer function of the UWB matched filter, which depends on the used pulse waveform. $|M(f)|$ can be evaluated as

$$
\begin{aligned}
M(f) & =F \cdot T \cdot\left\{p\left(t_{o}-t\right)-p\left(t_{o}-t-\delta\right)\right\} \\
& =F \cdot T \cdot\left\{m\left(t_{o}-t\right)\right\}
\end{aligned}
$$

where F.T. denotes the Fourier Transform, $\mathrm{p}(\mathrm{t})$ is the UWB pulse waveform, $t_{o}$ is the sampling instant and $\mathrm{m}(\mathrm{t})$ is the UWB correlation mask. 


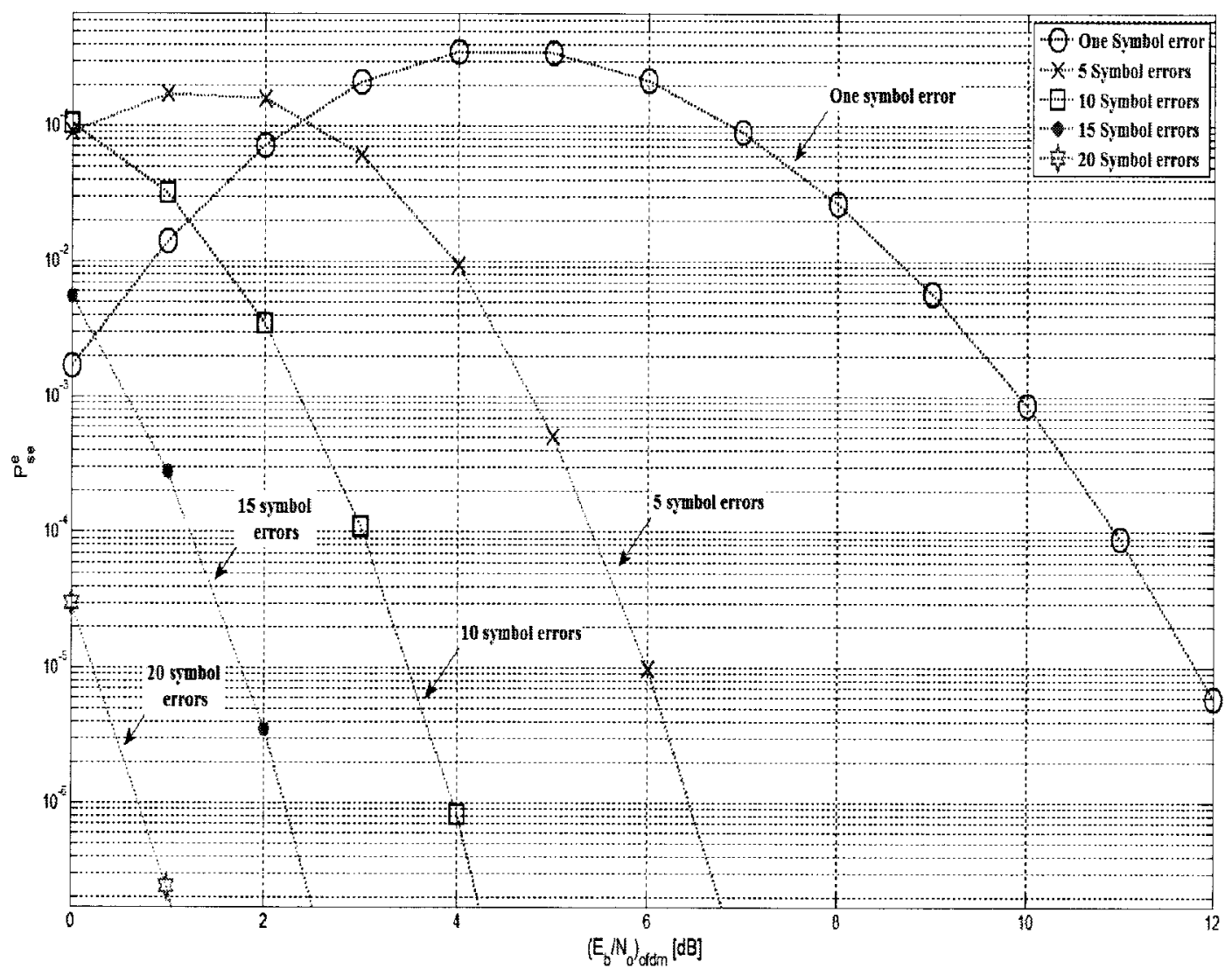

Figure 3.5: Probability of e symbol errors in a block of length $\mathrm{N}=52$ at $\mathrm{SIR}=0 \mathrm{~dB}$ and $B_{u w b} / B_{o f d m}=125$. 
For a TH-PPM system, the transfer function $|M(f)|$ is given by [27]

$$
M(f)=2 \cdot|P(f)| \cdot|\sin (\pi f \delta)| \cdot\left|\sum_{k=0}^{N_{s}-1} \exp \left[j 2 \pi f\left(k T_{f}+c_{k} T_{c}\right)\right]\right|
$$

whereas for a DS-BPSK modulation scheme, it can be written as [27]

$$
M(f)=2 \cdot|P(f)| \cdot\left|\sum_{k=0}^{N_{s}-1} c_{k} \cdot \exp \left[j 2 \pi f k T_{f}\right]\right|
$$

where $P(f)$ is the fourier Transform of the pulse $\mathrm{p}(\mathrm{t})$.

For a second derivative Gaussian mono-cycle, $\mathrm{p}(\mathrm{t})$ can be written as $[26]$

$$
p(t)=\varepsilon\left[1-4 \pi\left(\frac{t}{\tau_{p}}\right)^{2}\right] \exp \left[-2 \pi\left(\frac{t}{\tau_{p}}\right)^{2}\right]
$$

where $\varepsilon$ is introduced to normalize the energy of the pulses. For a Gaussian monocycle with energy $1 / N_{s}, \varepsilon=\sqrt{\frac{8}{3 N_{s} \tau_{p}}}[21]$.

The Fourier Transform of the received UWB pulse waveform is given by:

$$
P(f)=\sqrt{\frac{8}{3 N_{s} \tau_{p}}} \cdot \frac{\pi}{\sqrt{2}} \tau_{p}^{3} f^{2} \exp \left(-\frac{\pi f^{2} \tau_{p}^{2}}{2}\right)
$$

It has been suggested in [27] that the standard IEEE802.11a NBI signal can be approximated as sum of $N_{i}$ tone interferers.

Equation (3.8) can be rewritten to include the number of interfering tones $N_{i}$ as

$$
\begin{aligned}
P_{e}=Q\left(\sqrt{\frac{E_{b}}{N_{o}} \cdot(1-\varrho)}\right)+1 / \pi \int_{0}^{\infty} & {\left[1-\prod_{n=1}^{N_{i}} J_{o}\left(\omega \sqrt{\frac{1}{S I R} \cdot \frac{\left|M\left(f_{n}\right)\right|^{2}}{N_{i} \cdot T_{b}} \cdot \frac{2}{(1-\varrho)^{2}}}\right)\right] } \\
& \frac{\sin (\omega)}{\omega} \cdot \exp \left(-\frac{\omega^{2}}{2} \cdot \frac{N_{o}}{E_{b}} \cdot \frac{1}{(1-\varrho)}\right) d \omega
\end{aligned}
$$

We assume that the IEEE802.11a NBI signal operates in the U-NII upper band with center frequency $f_{c}=5.745 \mathrm{GHz}$ and approximated by $N_{i}=52$ data sub-carriers with carrier spacing $\Delta f=0.3125 \mathrm{MHz}$. Figure 3.6 depicts the probability of error for the TH-PPM system in the presence of the standard IEEE802.11a NBI signal. 
A "6th" derivative Gaussian mono-cycle is used, which is given by [27]

$$
p(t)=\sqrt{\frac{640}{231 N_{s} \tau_{p}}}\left[1-12 \pi\left(\frac{t}{\tau_{p}}\right)^{2}+16 \pi^{2}\left(\frac{t}{\tau_{p}}\right)^{4}-\frac{64}{15} \pi^{3}\left(\frac{t}{\tau_{p}}\right)^{6}\right] \exp \left[-2 \pi\left(\frac{t}{\tau_{p}}\right)^{2}\right]
$$

where $\tau_{p}=0.192 \mathrm{~ns}, N_{s}=4$ pulses per information bit, a frame duration $T_{f}=100$ ns, a correlation parameter $\varrho=-0.824$, a pulse position offset $\delta=0.068 \mathrm{~ns}$, for a user with TH sequence $\left\{c_{k}\right\}=\{0,10,5,20\}$ and chip duration $T_{c}=0.5$ ns.

Its Fourier Transform can be written as [27]

$$
P(f)=\frac{8 \pi^{3}}{3 \sqrt{1155 N_{s}}} \cdot \tau_{p}^{13 / 2} f^{6} \exp \left(-\frac{\pi f^{2} \tau_{p}^{2}}{2}\right)
$$

Figure 3.6 shows how the UWB system is affected by the IEEE802.11a interference at different SIRs. The SNR degradation is expected to reach up to $4 \mathrm{~dB}$ by changing the SIR from $0 \mathrm{~dB}$ to $-25 \mathrm{~dB}$ at $\mathrm{BER}=1 \times 10^{-3}$ for a UWB data rate equals to 2.5 Mbps.

When the NBI receiver introduces one symbol error, the BER probability of the UWB system can be evaluated using equation (3.14) and choosing $\left(N_{i}=1\right)$. By substituting in equation (3.5), the BER probability of the canceller scheme is calculated and shown in figure 3.7 .

In this figure, the performance of the canceller scheme is compared with the performance of a UWB system under the impact of the IEEE802.11a NBI at SIRs = -20 and $-25 \mathrm{~dB}$. It can be seen that the ICS mitigates the impact of the NBI signal and enhances the performance of the UWB communication system. For a $\mathrm{SIR}=-25$ $\mathrm{dB}$ and at $\mathrm{BER}=1 \times 10^{-4}$, the SNR degradation is reduced by $10 \mathrm{~dB}$ with the use of the canceller scheme.

It is worth mentioning that, the NBI receiver average probability of OFDM symbol error (av. Pse) can be considered as one of the main factors that controls the performance of such canceller scheme. By manipulating this parameter we can evaluate the ICS performance.

The performance of the ICS is depicted in figure 3.8 when the NBI demodulator introduces multiple symbol errors $e=1: N_{i}, N_{i} \in[2,52]$ and for an av. Pse $=0.1$. 


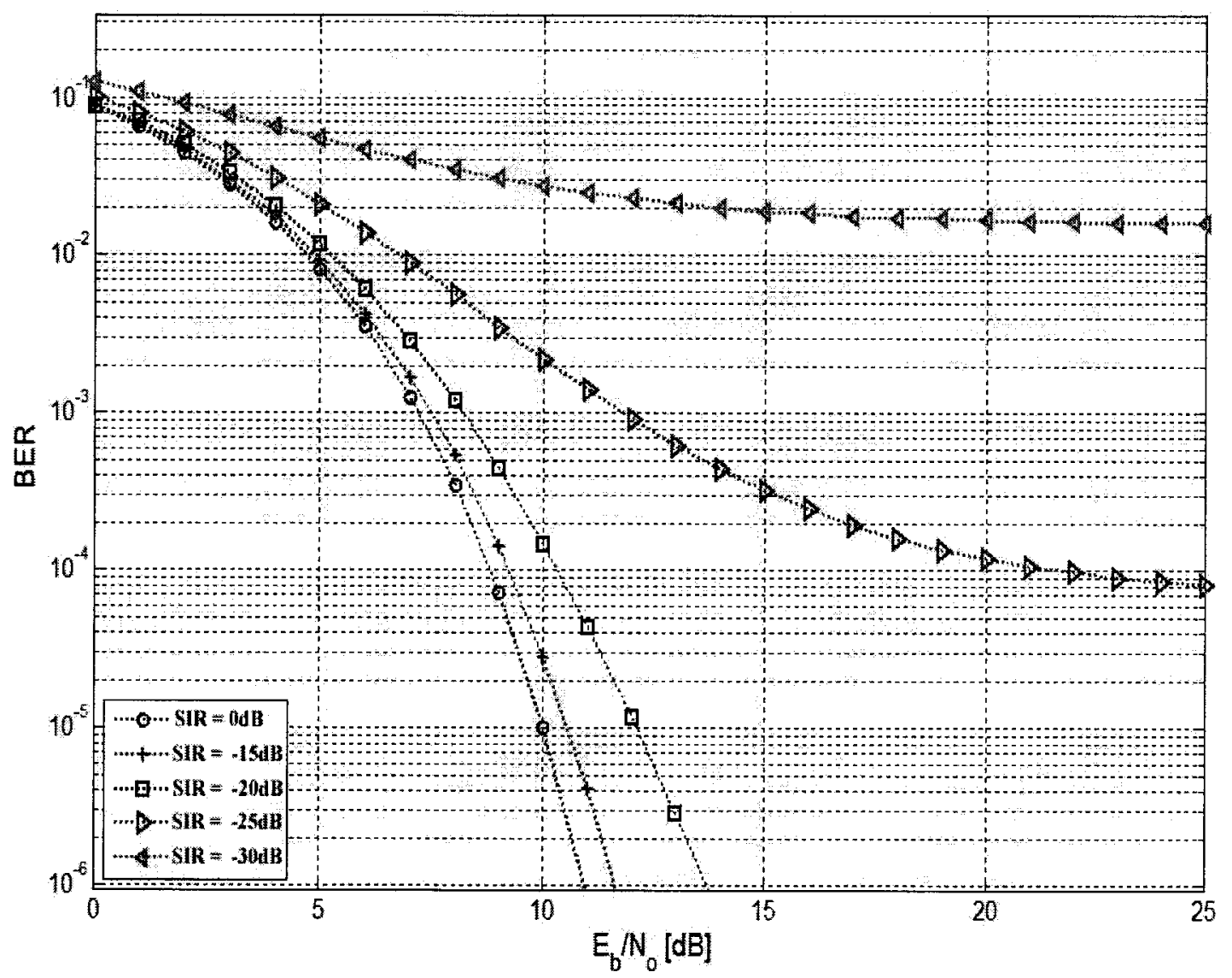

Figure 3.6: BER performance of the TH-PPM system in the presence of $N_{i}=52$ tone interferers. 
The data rate for the UWB system is $R_{b}=100 \mathrm{Mbps}$, with the same parameters stated previously at different SIR ratios $0,-15$ and $-20 \mathrm{~dB}$. It can be seen that, for a SIR $=-15 \mathrm{~dB}$, the performance of the UWB communication system is severely deteriorated by the presence of the NBI signal. However, the SNR degradation is reduced to be less than $3 \mathrm{~dB}$ at $\mathrm{BER}=1 \times 10^{-5}$ with the use of the ICS.

\subsection{Performance of the ICS in Nakagami-m Flat Fading Channels}

The performance of the canceller scheme is analyzed under the assumption of flat fading. The performance of a UWB system in the presence of NBI approximated by " $N_{i}$ " tones in a flat fading environment for both the desired UWB and interference signals is evaluated in [71]. It was assumed that the UWB signal undergoes a Nakagami-m fading, whereas the narrowband interferers originate from other sources, which could be affected by Rayleigh fading.

It was reported in [71] that these tones with arbitrary amplitudes and frequencies have independent Rayleigh fading tone by tone. The average BER of the UWB system under the assumption of perfect channel estimation is given by

$$
P_{e}=\frac{\Gamma(m+1 / 2)}{2 \sqrt{\pi} \Gamma(m+1)}\left(\frac{m}{\eta}\right)^{m}{ }_{2} F_{1}\left(m, m+1 / 2 ; m+1 ;-\frac{m}{\eta}\right)
$$

where ${ }_{2} F_{1}(., ; ; . ;$.$) is the Gauss hypergeometric function [72]{ }^{7}, \mathrm{~m}>0.5$ is the fading parameter that controls the severity of the fading conditions. $\Gamma($.$) is the gamma$ function and $\eta$ is the average SNIR, which is given by:

$$
\eta=\left(\frac{N_{o}}{E_{b}} \cdot \frac{2}{1-\varrho}+\frac{2}{(1-\varrho)^{2}} \sum_{n=1}^{N_{i}} \frac{1}{S I R \cdot N_{i}} \cdot \frac{\left|M\left(f_{n}\right)\right|^{2}}{T_{b}}\right)^{-1}
$$

Where $|M(f)|$ can be represented by equation (3.10) for a second derivative Gaussian pulse.

\footnotetext{
${ }^{7}$ Page 1010, Equation (9.14).
} 


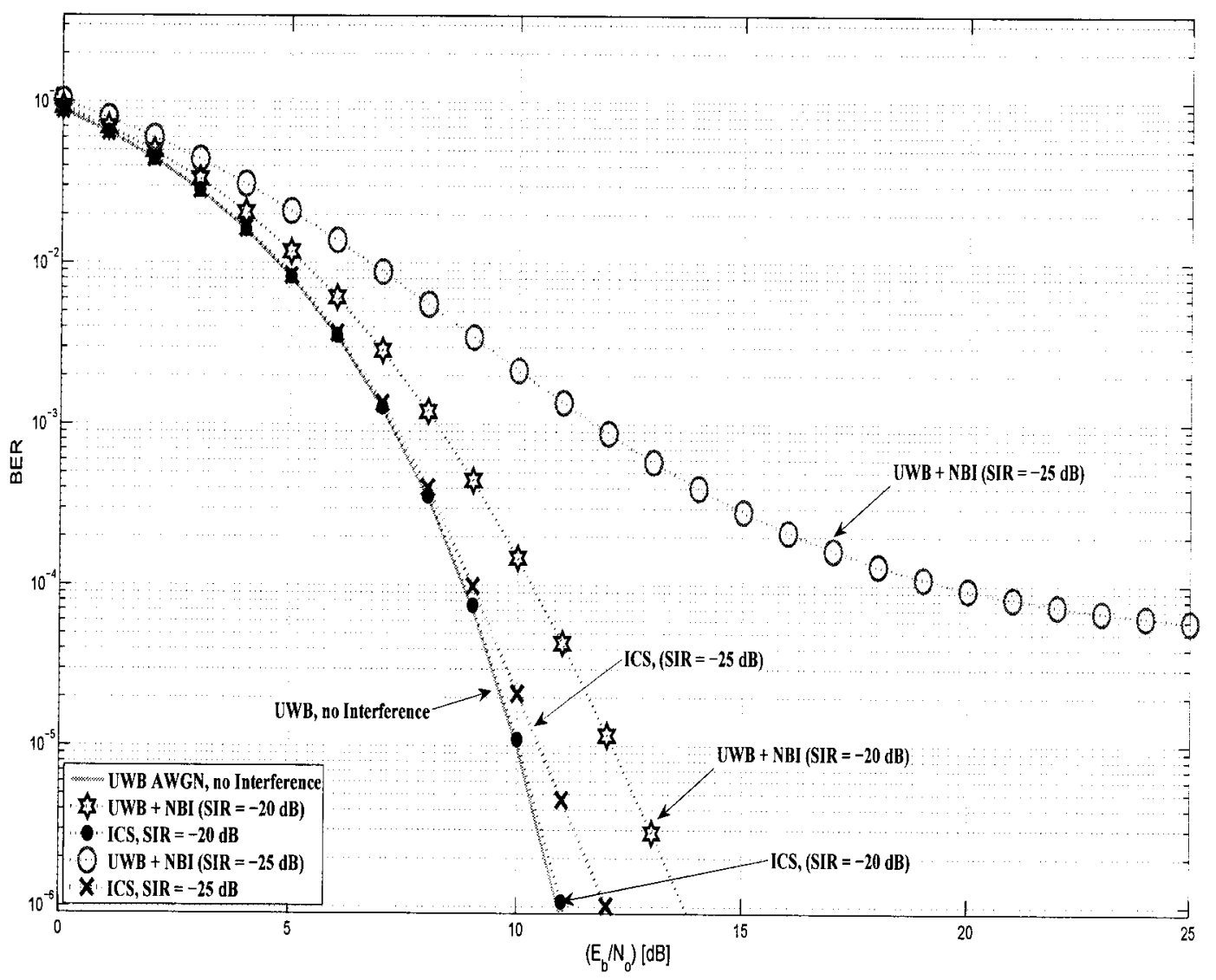

Figure 3.7: BER performance of the ICS for one symbol error case (one tone interferer). 


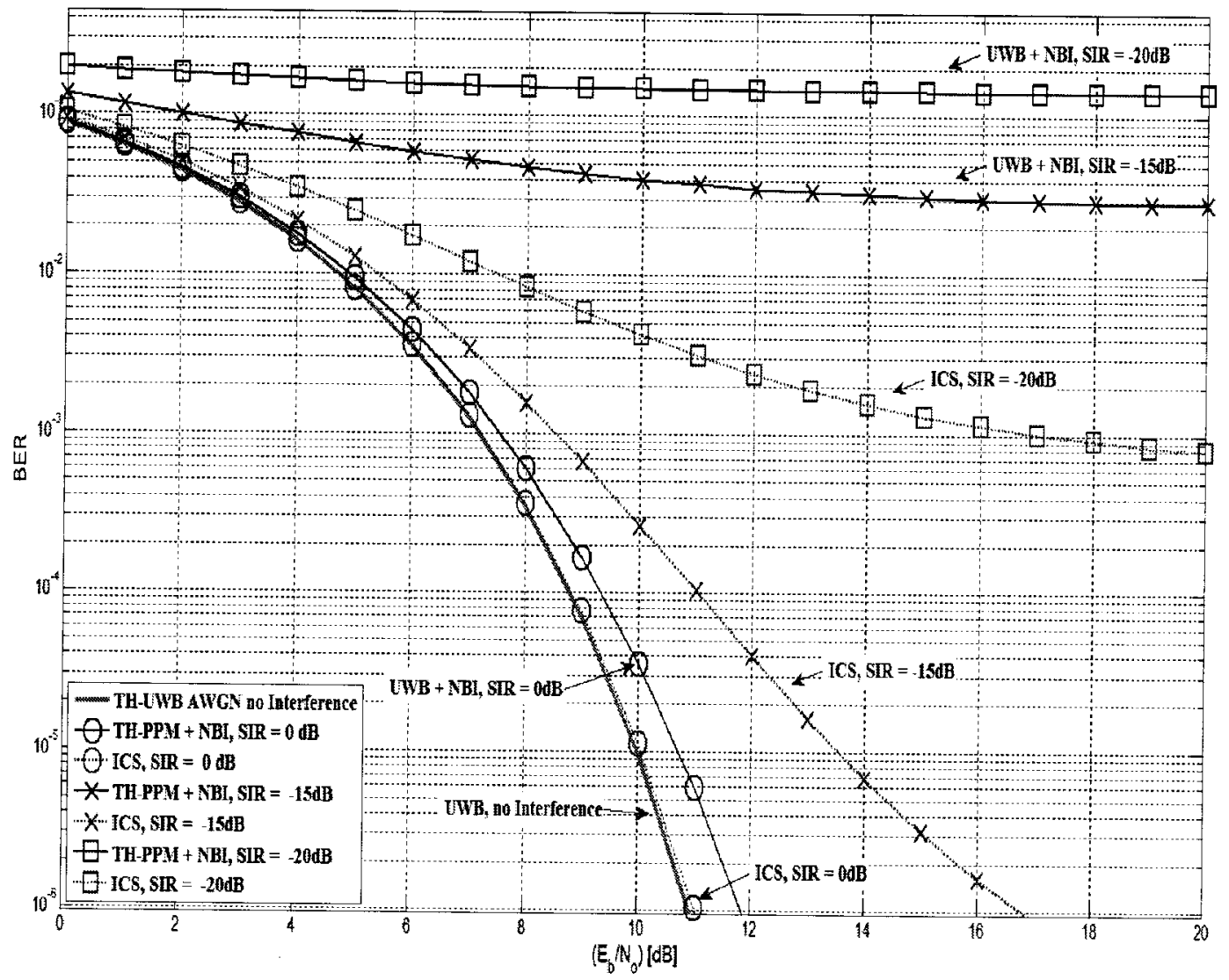

Figure 3.8: BER performance of the ICS for multiple symbol errors case. 
For integer values of $m$, the UWB average BER performance can be simplified as [71]

$$
P_{e}=\left[\frac{1}{2}-\frac{1}{2}\left(1+\frac{m}{\eta}\right)^{-\frac{1}{2}}\right]^{m} \cdot \sum_{j=0}^{m-1}\left(\frac{(m-1+j) !}{(m-1) !(j) !}\right)\left[\frac{1}{2}+\frac{1}{2}\left(1+\frac{m}{\eta}\right)^{-\frac{1}{2}}\right]^{j}
$$

Figure 3.9 depicts the performance of a TH-PPM system in the presence of the IEEE802.11a interference signal in a Nakagami-m flat fading channel. Different values of the Nakagami fading parameter $m$ and different UWB data rates $\left(R_{b}=25 \mathrm{Mbps}\right.$ and $100 \mathrm{Mbps}$ ) at $\mathrm{SIR}=-10 \mathrm{~dB}$ have been used. As expected, the performance of the TH-PPM system is severely degraded by the presence of NBI and fading on the desired UWB signal.

Figures 3.10 and 3.11 depict a comparison between the performance of the TH-PPM system with/without the use of the canceller scheme at different SIRs for an av. Pse equals to 0.1 for a Nakagami fading parameter $m=2$ and 8 respectively.

It can be seen that for $\mathrm{m}=2$ and for $R_{b}=25 \mathrm{Mbps}$, the SNR degradation is reduced to be less than $2 \mathrm{~dB}$ at $\mathrm{BER}=1 \times 10^{-3}$, whereas for $R_{b}=100 \mathrm{Mbps}$, the degradation is reduced to be less than $4 \mathrm{~dB}$ at $\mathrm{BER}=5 \times 10^{-3}$. When the Nakagami fading parameter $\mathrm{m}=8$, the SNR degradation is expected to be less than $1 \mathrm{~dB}$ at $\mathrm{BER}=1 \times 10^{-4}$ and less than $2 \mathrm{~dB}$ at $\mathrm{BER}=1 \times 10^{-3}$ for UWB data rates $25 \mathrm{Mbps}$ and 100 Mbps respectively.

\subsection{Summary of the ICS BER Performance}

In this section, we present a summary of the BER performance of the ICS based on evaluating its performance at different values of the NBI receiver average probability of OFDM symbol error.

Figure 3.12 depicts the BER performance of the ICS in AWGN channel environment and for SIR $=-20 \mathrm{~dB}$. It can be seen that, although the UWB system is complctely jammed by the NBI signal, the canceller scheme can efficiently suppress the impact of such NBI signal at an av. Pse $=1 \times 10^{-3}$. In this case, the performance of the UWB system with the ICS is equivalent to the performance of a UWB system as if there was no interference. For an av. Pse $=0.05$, the SNR degradation is reduced ${ }^{8}$ to be less than $2.5 \mathrm{~dB}$ at $\mathrm{BER}=1 \times 10^{-3}$.

\footnotetext{
${ }^{8}$ In comparison with the UWB performance in the presence of NBI without the use of the ICS.
} 


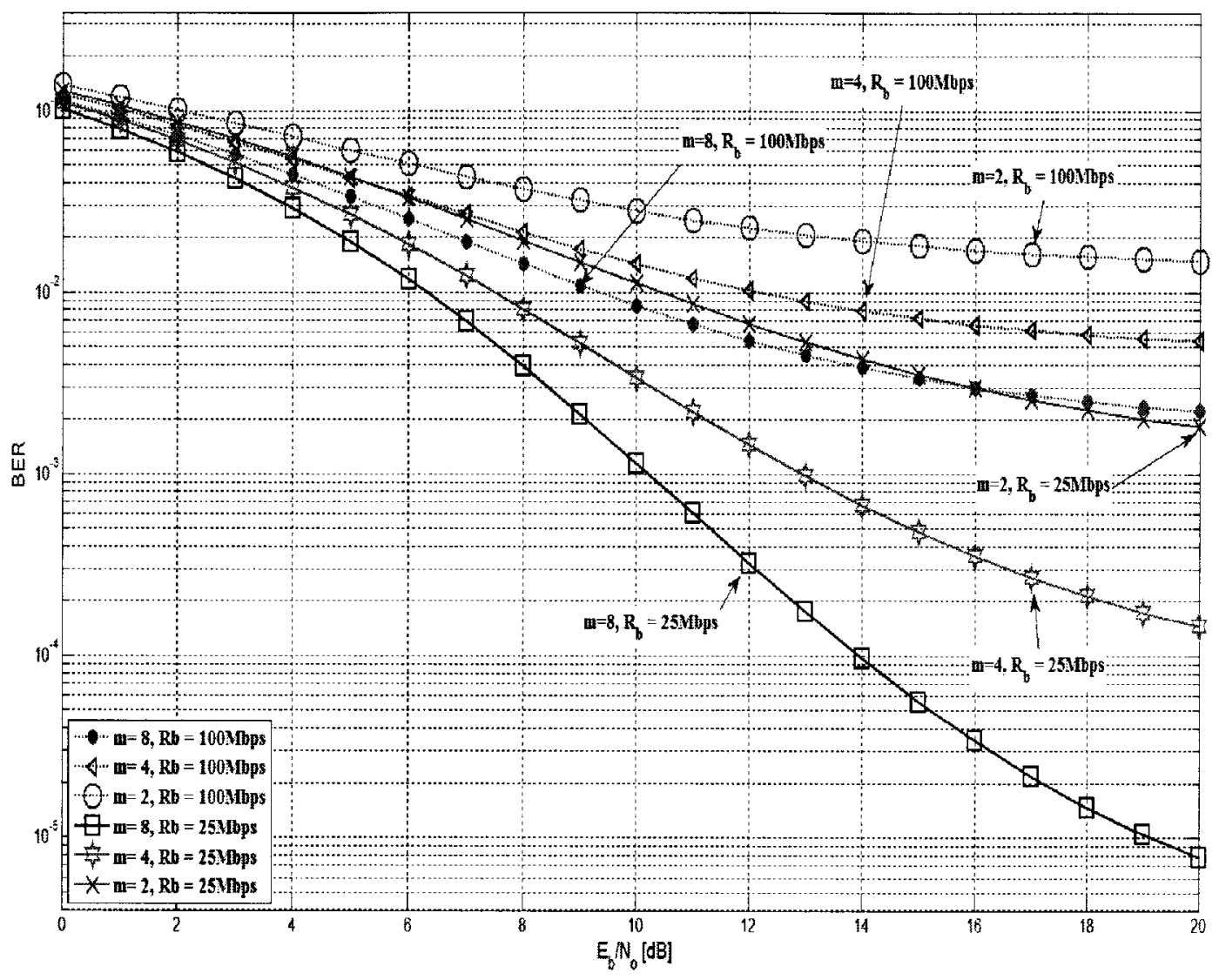

Figure 3.9: BER performance of a UWB system in presence of IEEE802.11a NBI signal for different data rates and different values of the Nakagami fading parameter $\mathrm{m}, \mathrm{SIR}=-10 \mathrm{~dB}$.

Figure 3.13 depicts also the BER performance of the ICS in a Nakagami-m flat fading channel with fading parameter $\mathrm{m}=2$. It can be seen that, the impact of NBI can be mitigated with the use of the ICS at an av. Pse $=1 \times 10^{-3}$. While, for an av. Pse $=$ 0.05 , the SNR degradation is reduced to be less than $4 \mathrm{~dB}$ at $\mathrm{BER}=1 \times 10^{-2}$. 


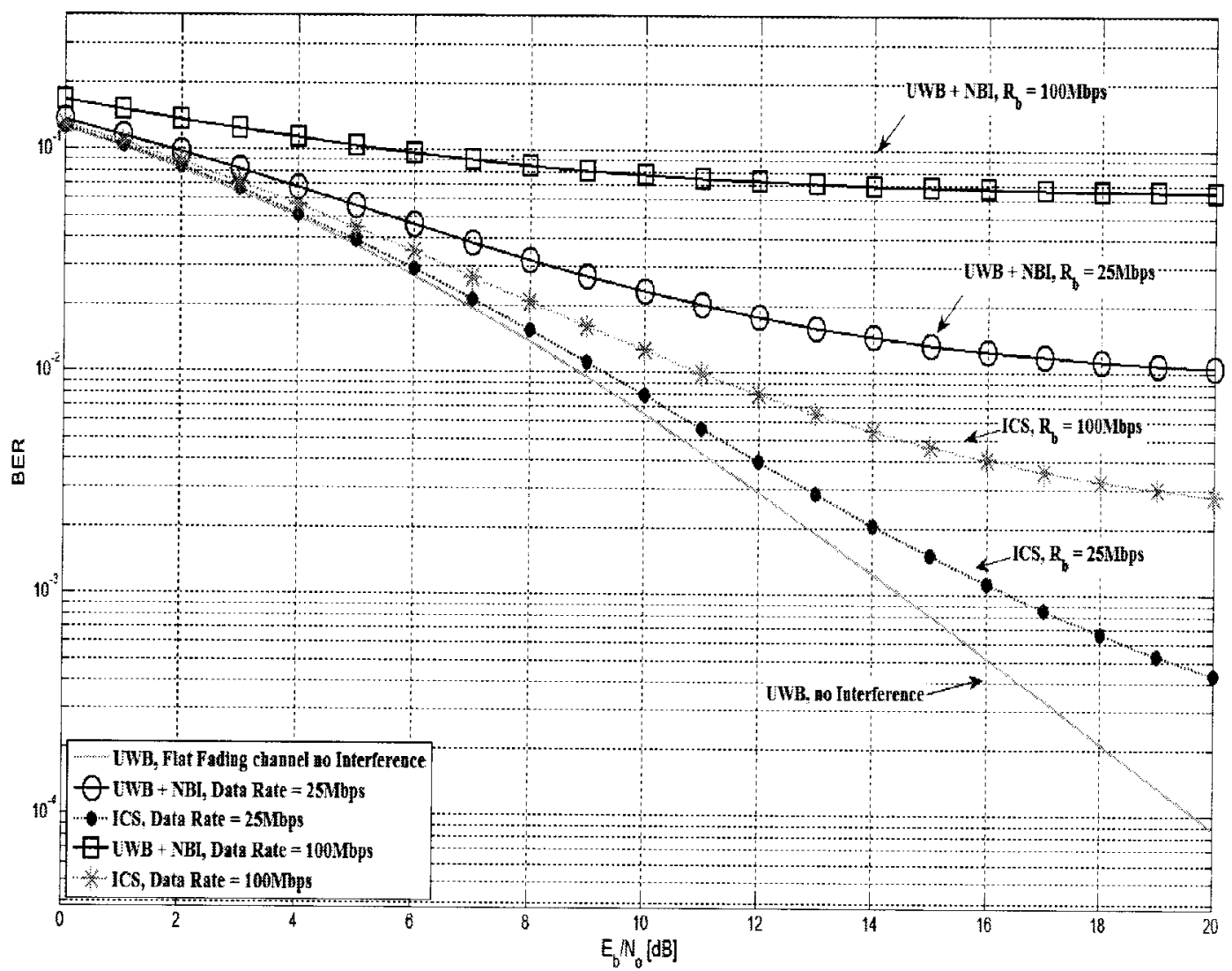

Figure 3.10: UWB BER performance comparison with/without the ICS in a Nakagami flat fading channel, $\mathrm{m}=2$, av. $P_{s e}=0.1$ and $\mathrm{SIR}=-15 \mathrm{~dB}$. 


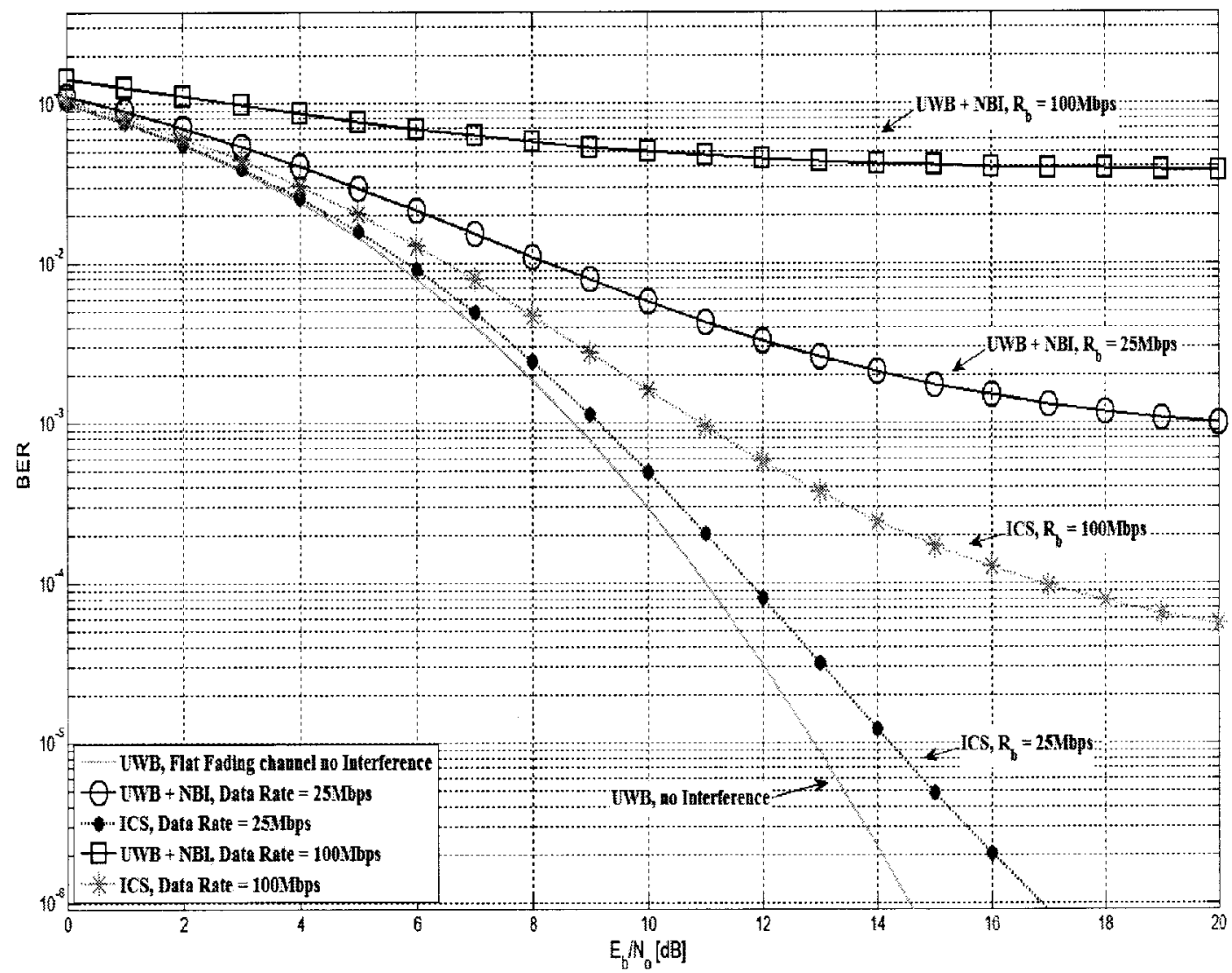

Figure 3.11: UWB BER performance comparison with/without the ICS in a Nakagami flat fading channel, $\mathrm{m}=8$, av. $P_{s e}=0.1$ and $\mathrm{SIR}=-15 \mathrm{~dB}$. 


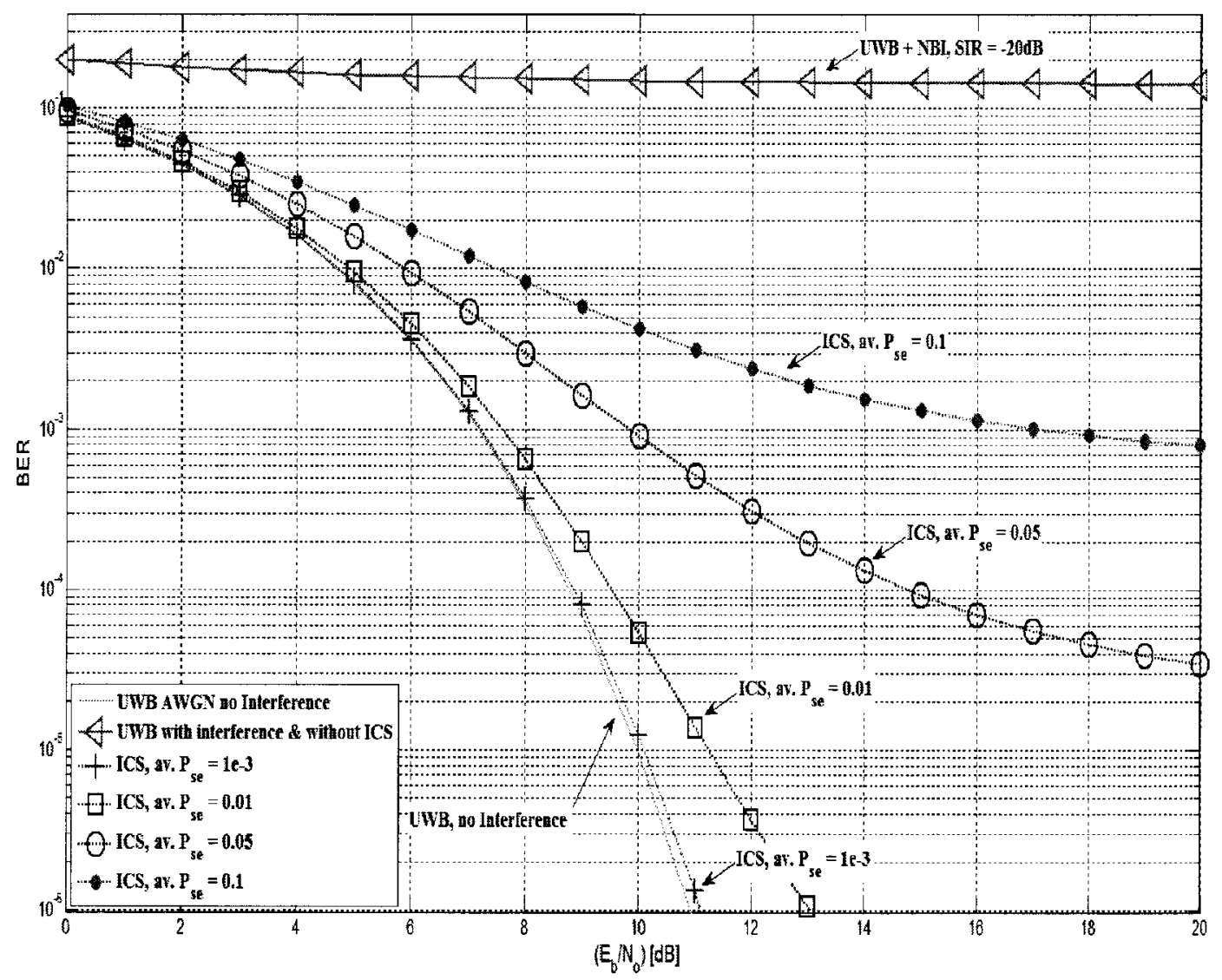

Figure 3.12: ICS BER performance in AWGN channel, $\mathrm{SIR}=-20 \mathrm{~dB}$. 


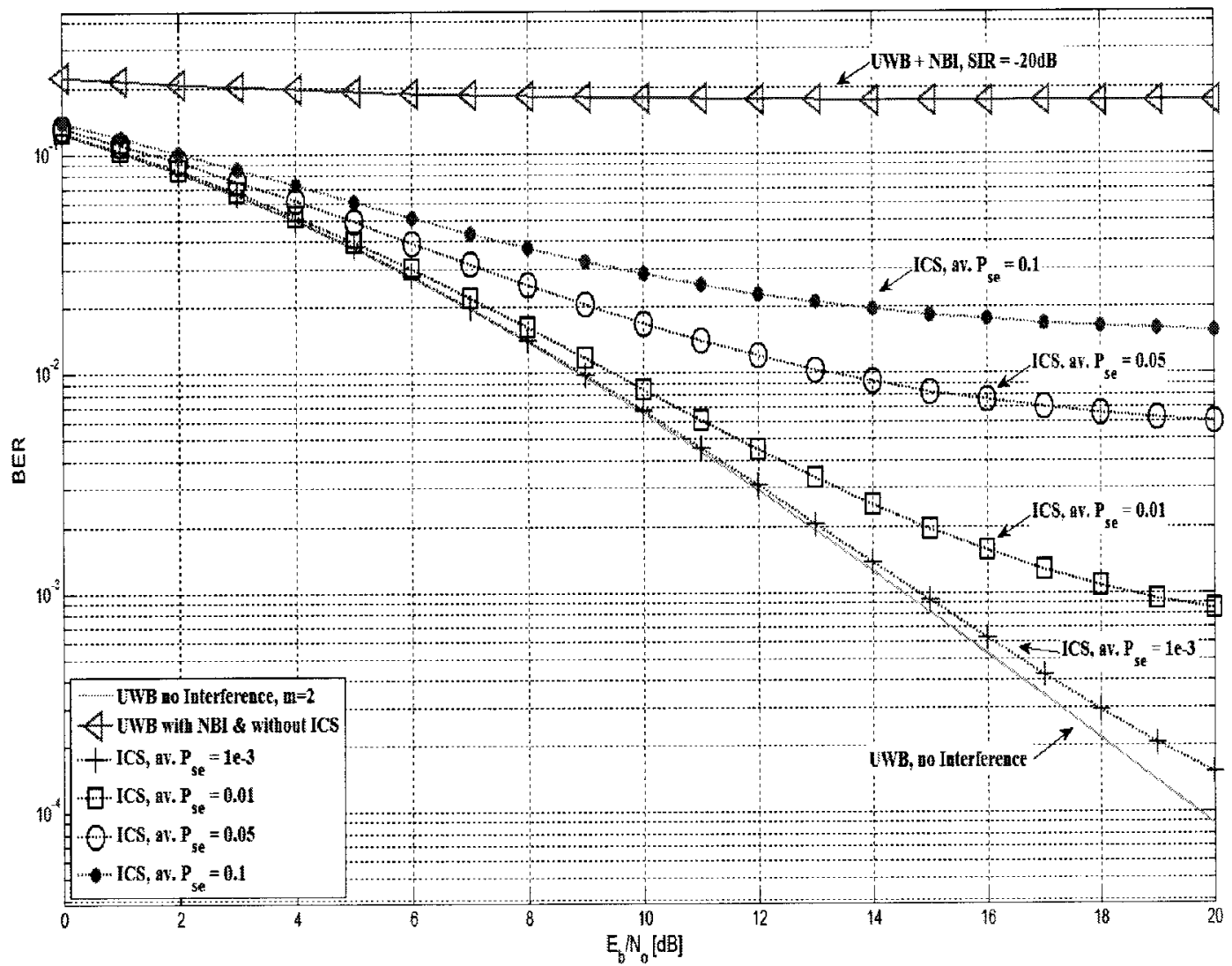

Figure 3.13: ICS BER performance in a Nakagami-m flat fading channel, SIR = $-20 \mathrm{~dB}$. 


\section{Chapter 4}

\section{Simulation of the ICS}

In this chapter, the simulation procedures and blocks that had been used to simulate the performance of the ICS in various fading channels environments is presented. Initially, the main simulation blocks for both the desired UWB and NBI systems are presented. Subsequently, the simulation blocks of the ICS are presented. Finally, comparisons between the obtained analytical results and the simulation ones in different channel environments are presented. Through this chapter, all the simulation results were achieved with the aid of the Matlab software code ${ }^{1}$.

\subsection{Simulation of the IR-UWB Communication System}

\subsubsection{TH-PPM Transmission Scheme}

The transmission block diagram of the TH-PPM-UWB system is depicted in figure 4.1 [9], where the blocks that constitute the simulation block diagram can be summarized as:

\section{- Code Repetition Coder:}

The binary transmitted sequence $\mathbf{a}=\left(\ldots, a_{o}, a_{1}, a_{2}, \ldots\right)$ is generated at the required information rate, $R_{b}=\frac{1}{T_{b}}$ bits/sec. This binary sequence enters the code repetition coder block, which is responsible for producing redundancy by repeating each bit according to the required number of pulses transmitted per bit $N_{s}$.

\footnotetext{
${ }^{1}$ Matlab version 7.5.0.342 (R2007b).
} 


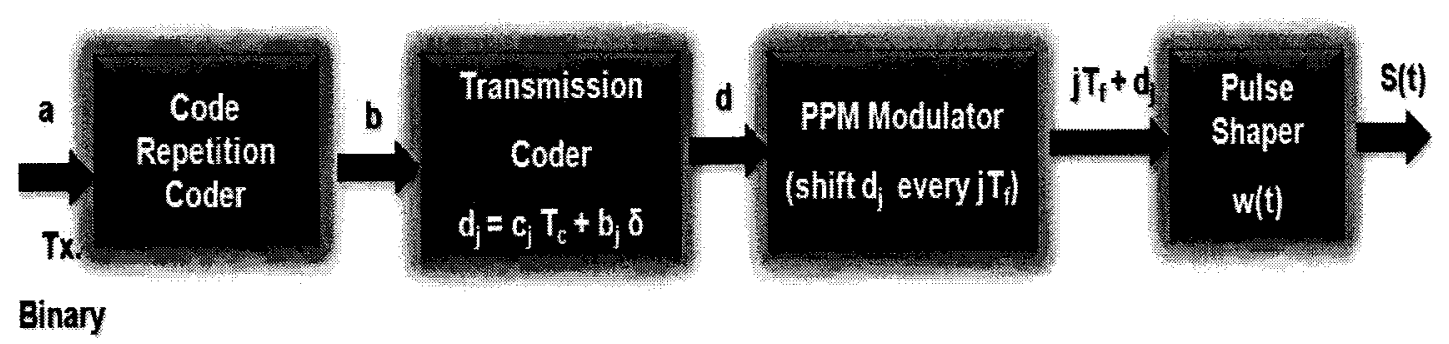

seq.

Figure 4.1: The Block diagram of the TH-PPM-UWB Transmission System [9].

The output from the code repetition coder block will be in the form, $\mathrm{b}=$ $\left(\ldots, b_{o}, \ldots, b_{o}, b_{1}, \ldots, b_{1}, b_{2}, \ldots, b_{2}, \ldots\right)$, at a rate $=\frac{1}{T_{f}} \mathrm{bits} / \mathrm{sec}$

\section{- Transmission Coder:}

The transmission coder block is responsible for generating a new sequence $\mathbf{d}$, by applying the pseudo random periodic integer valued $\mathbf{c}$ to the binary sequence b. The pseudo random code has a period $N_{p}$. The generic form of the new sequence $\mathbf{d}$ can be written as

$$
d_{j}=c_{j} T_{c}+b_{j} \delta
$$

\section{- PPM Modulator:}

This block generates unit Dirac pulses at times $\mathrm{j} T_{f}+c_{j} T_{c}+b_{j} \delta$. Another shift is introduced by the PPM modulator that is $b_{j} \delta$. This shift is usually much less than the TH shift $c_{j} T_{c}$ except for $c_{j}=0$.

\section{- Pulse Shaper:}

The final block is the pulse shaper, which is simply a filter with impulse response $\mathrm{w}(\mathrm{t})$ that has to be chosen carefully as it affects the power spectral density of the UWB transmitted signal. The most adapted pulse shapes are modeled as higher derivatives of a Gaussian pulse to meet the FCC spectral mask. 
The signal at the output of the pulse shaping filter can be written as

$$
S(t)=\sum_{j=-\infty}^{\infty} w\left(t-j T_{f}+c_{j} T_{c}+b_{j} \delta\right)
$$

\subsubsection{DS-BPSK Transmission Scheme}

The transmission scheme for the UWB system with DS-BPSK modulation is depicted in figure 4.2 and can be summarized $\mathrm{as}^{2}$ :

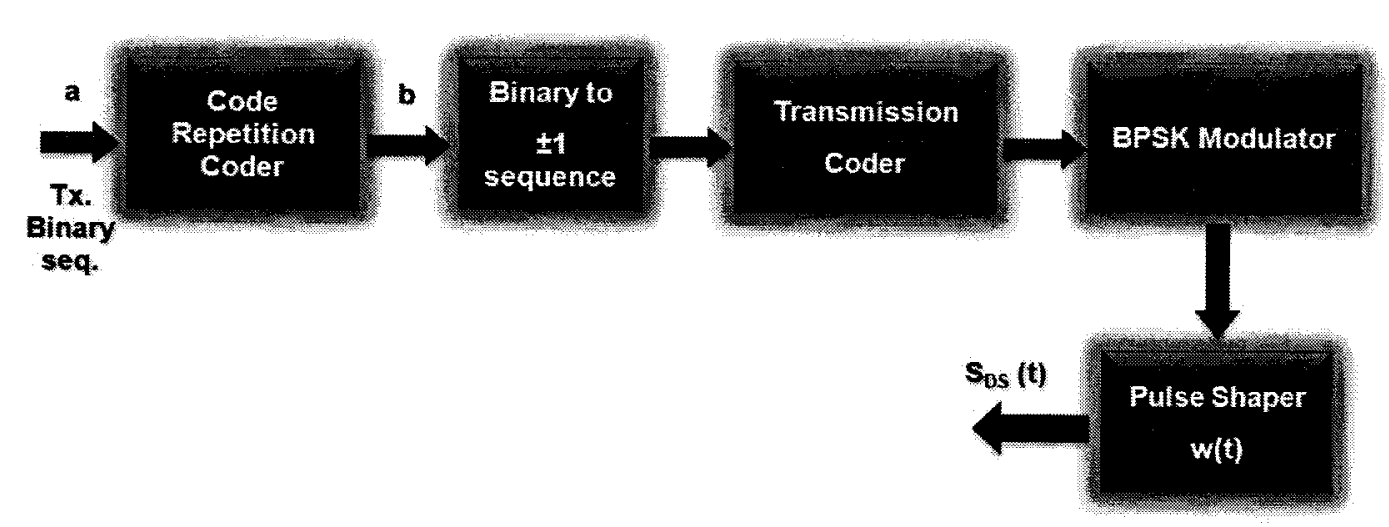

Figure 4.2: The Block diagram of the DS-BPSK-UWB Transmission System [9].

- Binary to \pm 1 Converter:

This block converts the input binary stream sequence into +1 and -1 series.

- Transmission Coder:

The transmission coder applies the binary code sequence $\mathrm{c}$ that is composed of \pm 1 's and period $N_{p}$ to the input sequence $\mathbf{d}$ to generate a new sequence $(\mathrm{g}=\mathrm{d} \cdot \mathrm{c})$.

\section{- BPSK Modulator:}

It generates a sequence of unit Dirac pulses at a rate of $\frac{1}{T_{f}}$ pulses $/ \mathrm{sec}$, which are located at times $j T_{f}$.

\footnotetext{
2 The code repetition coder and the pulse shaper blocks have the same function as presented in the TH modulation scheme.
} 


\subsubsection{IR-UWB Reception Scheme}

It is well known that the optimum receiver for the AWGN channel is a Matched Filter, which composed of a correlator and a detector. The optimum detector applies the Maximum likelihood (ML) criterion among the M possible transmitted waveforms.

The block diagram of the reception scheme of the UWB system is depicted in figure 4.3 .

$r(t)$

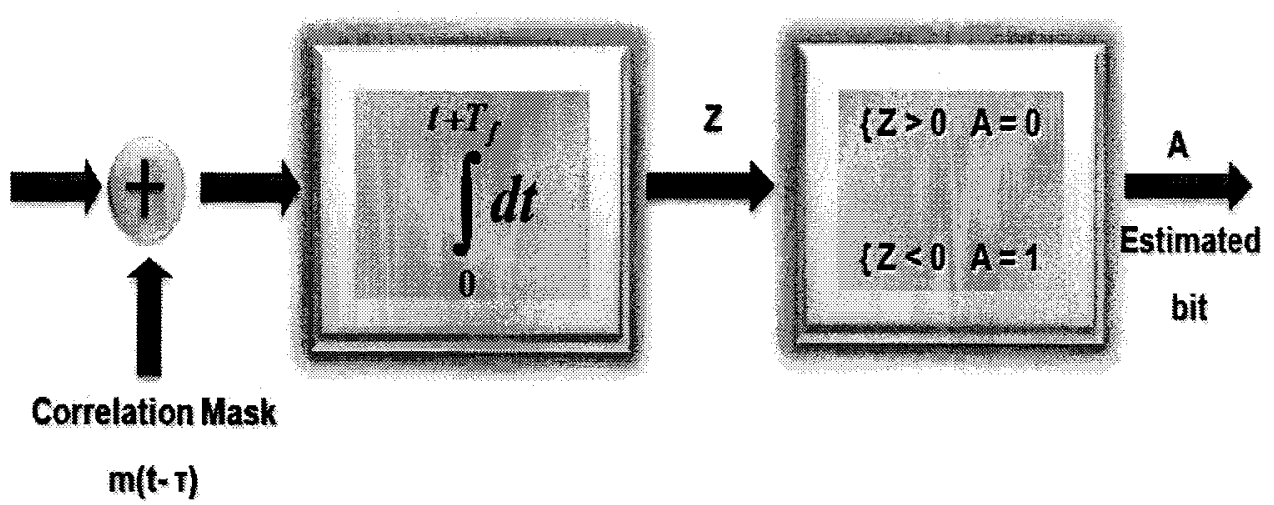

Figure 4.3: A single correlator UWB receiver scheme.

The depicted receiver scheme consists of a single correlator, where the incoming received signal is multiplied by the correlation mask $m(t-\tau) . \tau$ is the channel time delay.

The correlation mask for the TH-PPM system in AWGN channel can be written as:

$$
m(t)=p\left(t-\tau-c_{j} T_{c}\right)-p\left(t-\tau-c_{j} T_{c}-\delta\right)
$$

whereas, for the DS-BPSK system the correlation mask can be written as:

$$
m(t)=c_{j} p(t-\tau)
$$


The output of the correlator is given by:

$$
Z=\alpha \cdot s_{i}+n, \quad i=0,1
$$

with

$$
\left\{\begin{array}{lll}
\mathrm{s}_{o}=-\sqrt{E_{p}} & \text { and } \mathrm{s}_{1}=+\sqrt{E_{p}} & \text { for a TH-PPM } \\
\mathrm{s}_{0}=+\sqrt{E_{p}} & \text { and } \mathrm{s}_{1}=-\sqrt{E_{p}} & \text { for a DS-BPSK }
\end{array}\right.
$$

where $E_{p}$ is the transmitted energy per pulse, $\alpha$ is the channel gain and $\mathbf{n}$ is a Gaussian random variable with zero mean and variance $N_{o} / 2$.

Table 4.1 shows the used values of the simulated UWB parameters, unless otherwise stated.

Table 4.1: The used UWB simulated parameters value.

\begin{tabular}{|c|c|c|}
\hline Parameter & Symbol & Value \\
\hline \hline Pulse Shaping Factor & $\tau_{p}$ & $0.192 \mathrm{~ns}$ \\
\hline Pulse width & $T_{m}$ & $0.5 \mathrm{~ns}$ \\
\hline PPM delay & $\delta$ & $0.062 \mathrm{~ns}$ \\
\hline Chip width & $T_{c}$ & $1 \mathrm{~ns}$ \\
\hline Number of users & $N_{u}$ & 1 \\
\hline Frame width & $T_{f}$ & $4,8,10$ and $16 \mathrm{~ns}$ \\
\hline Tumber of pulses per frame & $N_{s}$ & $1,2 \mathrm{and} 4$ \\
\hline UWB pulses correlation coefficient & $\varrho$ & -0.824 \\
\hline Sampling rate & $f_{s}$ & $50 \mathrm{GHz}$ \\
\hline Order of differentiation & $\mathrm{n}$ & 6 \\
\hline
\end{tabular}

Figure 4.4 depicts a comparison between the simulation and analytical results to evaluate the BER performance of a TH-PPM system. It can be seen that a good agreement between the two results is achieved, in which we can conclude the validation of the simulation experiment.

Figure 4.5 also depicts this comparison in addition to the confidence intcrval of the simulation results. 


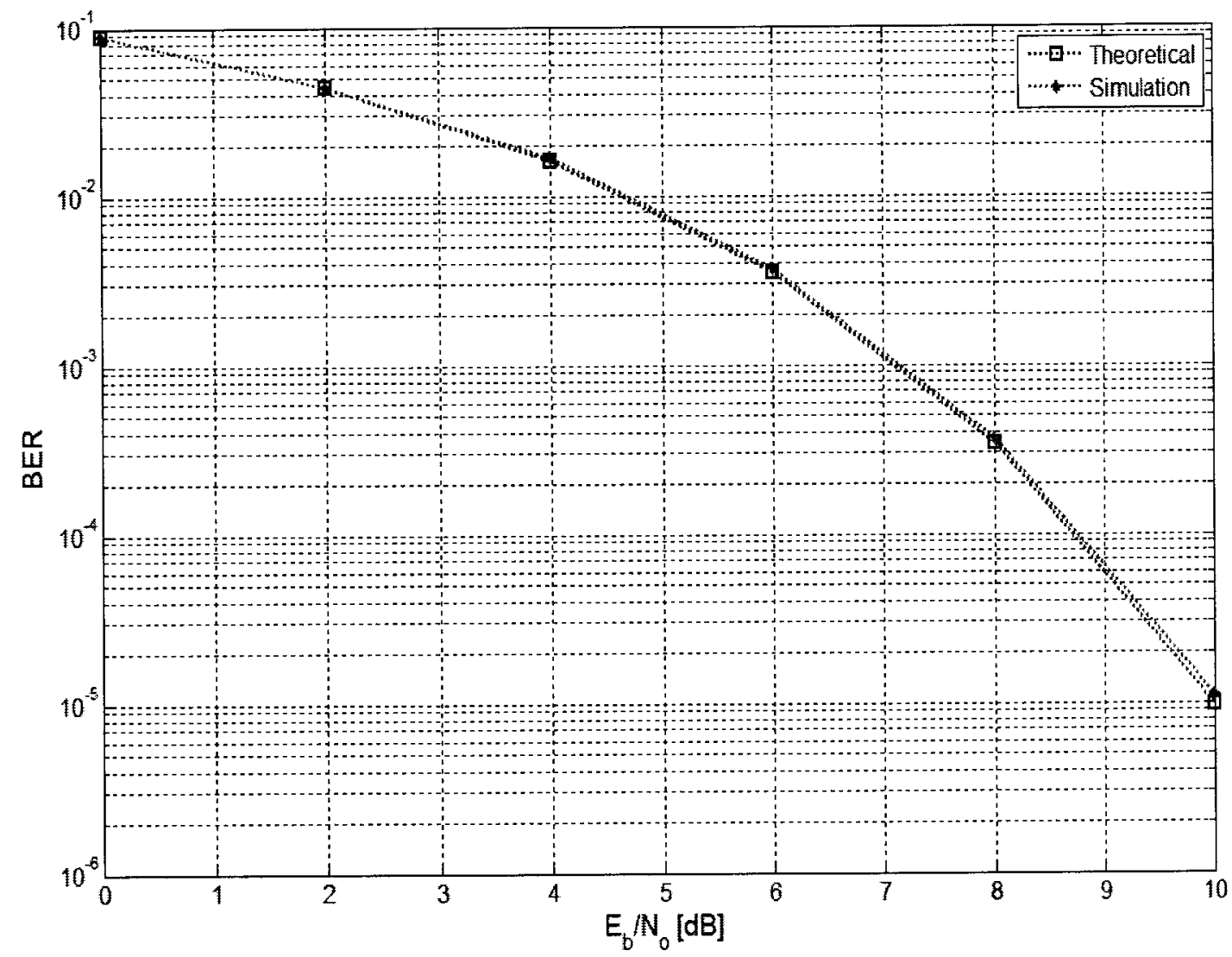

Figure 4.4: Simulation and Analytical evaluation of the BER performance of the TH-PPM system. 


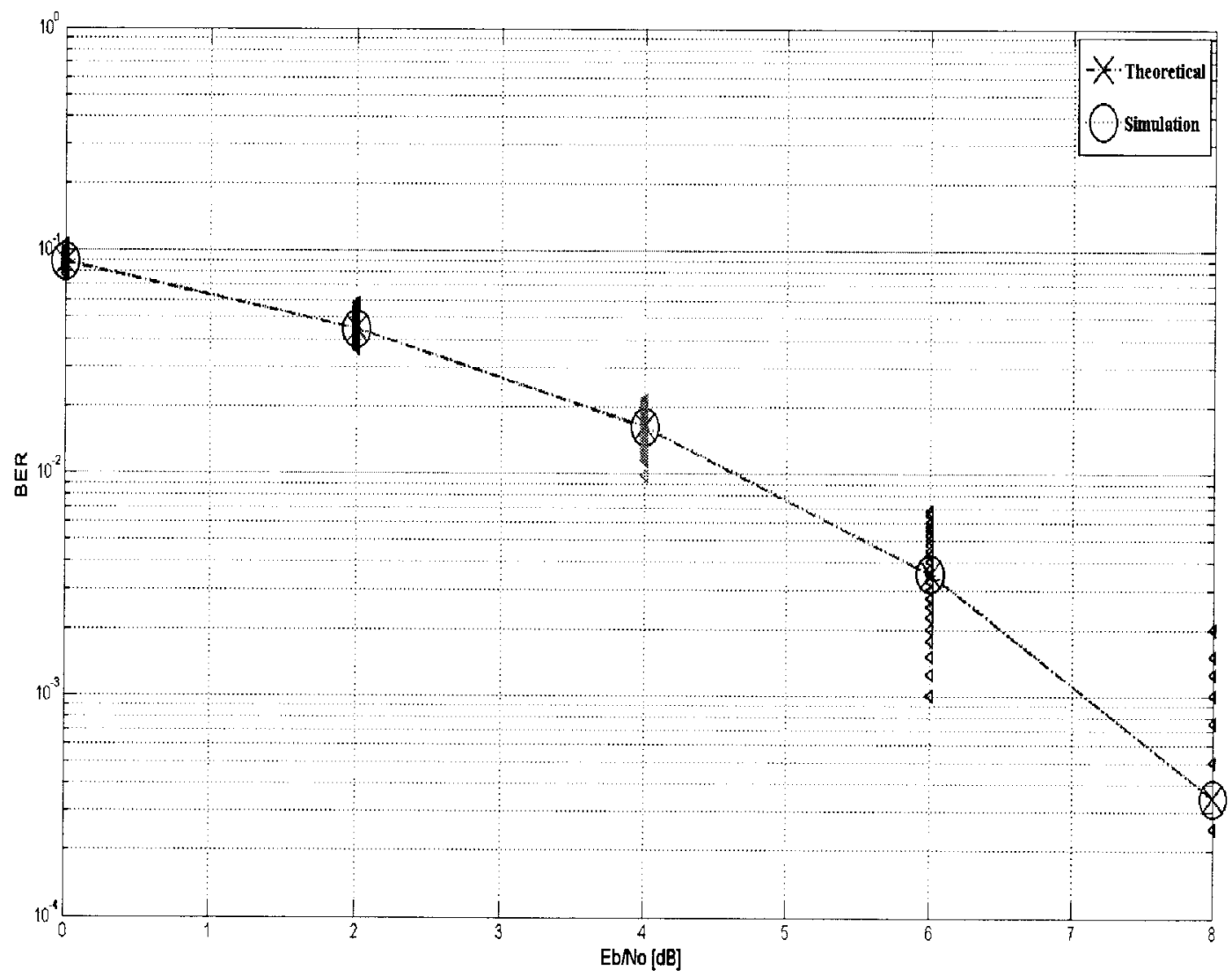

Figure 4.5: Simulation and Analytical evaluation of the BER performance of the TH-PPM system, showing the confidence interval of the simulation results. 


\subsubsection{Simulation of the IEEE802.15.3a UWB Propagation Channel}

A convenient model that characterizes multi-path fading channel is the discrete impulse response model suggested by [73]. In this model the time axis is divided into small time intervals "bins", which are assumed to contain either one or no multi-path component.

The impulse response can be written as

$$
h(t)=\sum_{n=1}^{N_{m}} \alpha_{n} \delta(t-n \Delta \tau)
$$

where, $N_{m}$ is the maximum number of bins considered within a single observation interval, and $\Delta \tau$ is the time duration of the bin.

In order to understand the performance of a UWB system in the IEEE802.15.3a channel models, simulations of the discrete time channel impulse response presented in equation (4.6) in the IEEE802.15.3a UWB four channel models (CM1, CM2, CM3, and CM4) are depicted in figures 4.6 and 4.7. It can be seen from figure 4.6 that as expected for the CM1 (LOS $0-4 \mathrm{~m}$ ) channel model, the first multi-path component gives the highest contribution of energy. The presence of different clusters is also not evident. For the CM2 (NLOS $0-4 \mathrm{~m}$ ) channel model, also as expected it can be observed that the first ray does not convey the highest energy, where the strongest rays arrive at the receiver after reflections or diffractions.

Whereas, in figure 4.7 , it can be seen that for the CM3 (NLOS $4-10 \mathrm{~m}$ ) channel model, where we can notice the presence of pulses in a time interval that is much longer than what has been presented in the CM1 and the CM2 channel models. For the CM4 (Extremely NLOS $4-10 \mathrm{~m}$ ) channel model, we can evident the presence of time dispersion of the transmitted energy, where a considerable concentration of received encrgy even after 50 ns from reception of the first contribution is observed. 


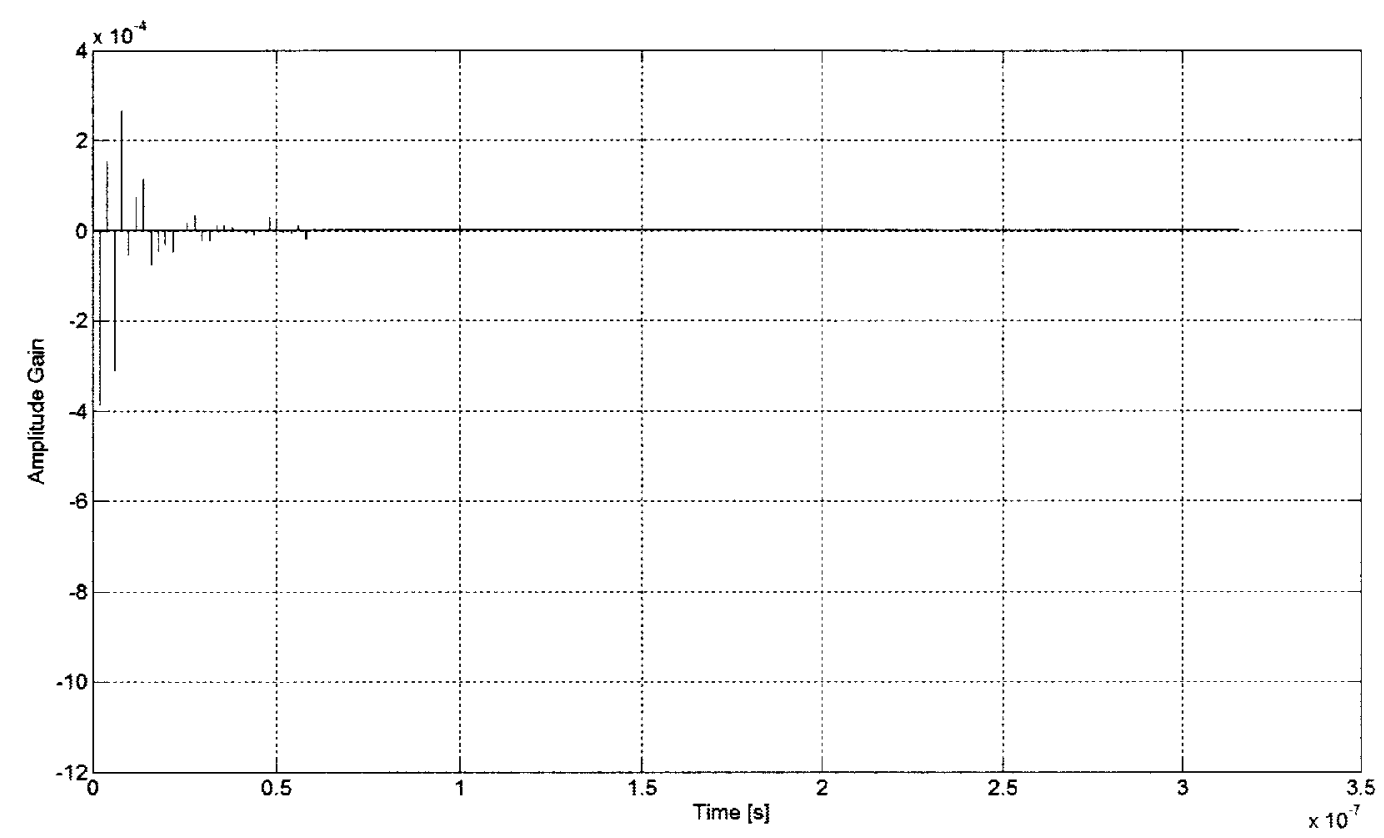

(a) CM1

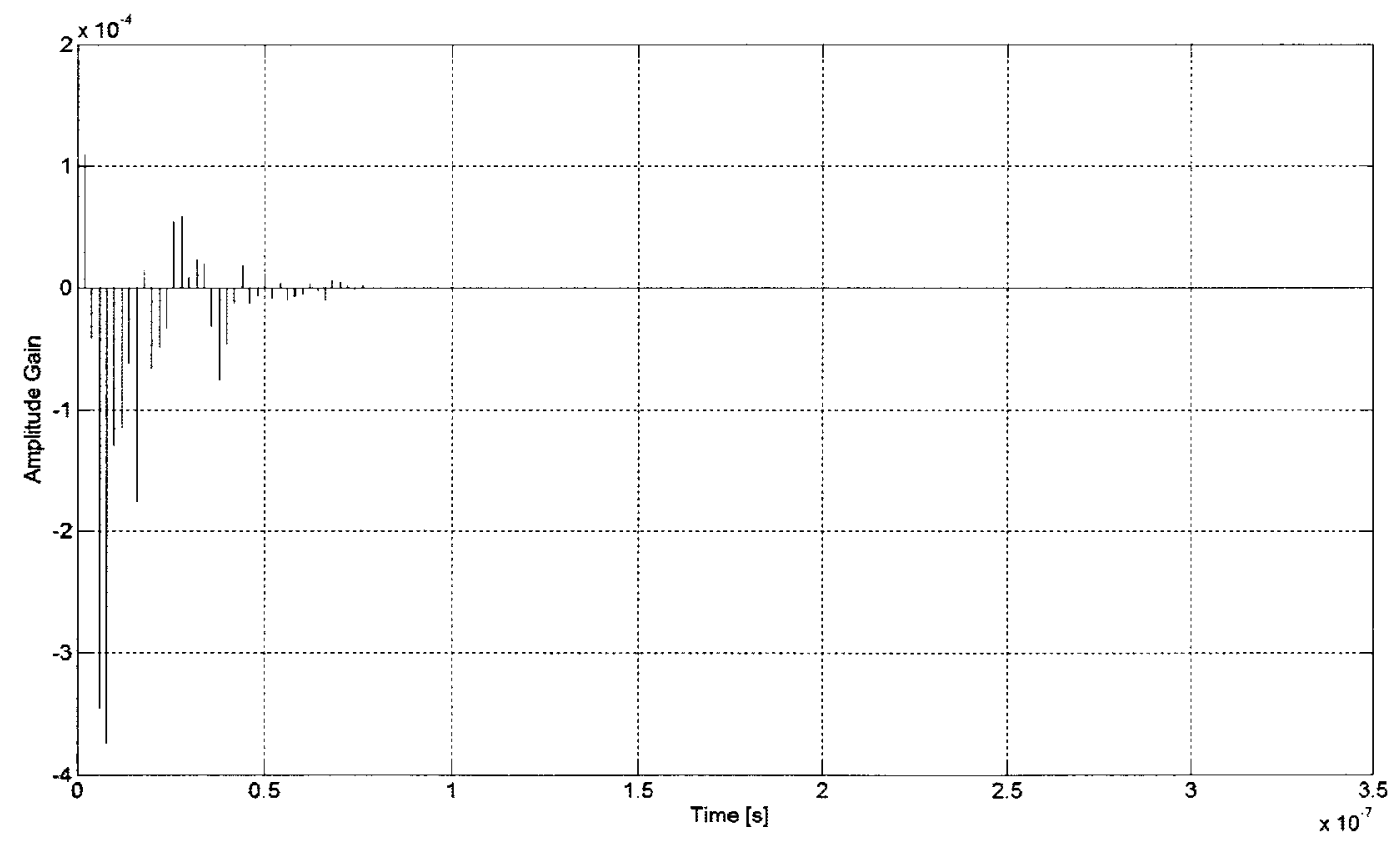

(b) CM2

Figure 4.6: A realization of the discrete time impulse response for the CM1 and the CM2 channel models. 


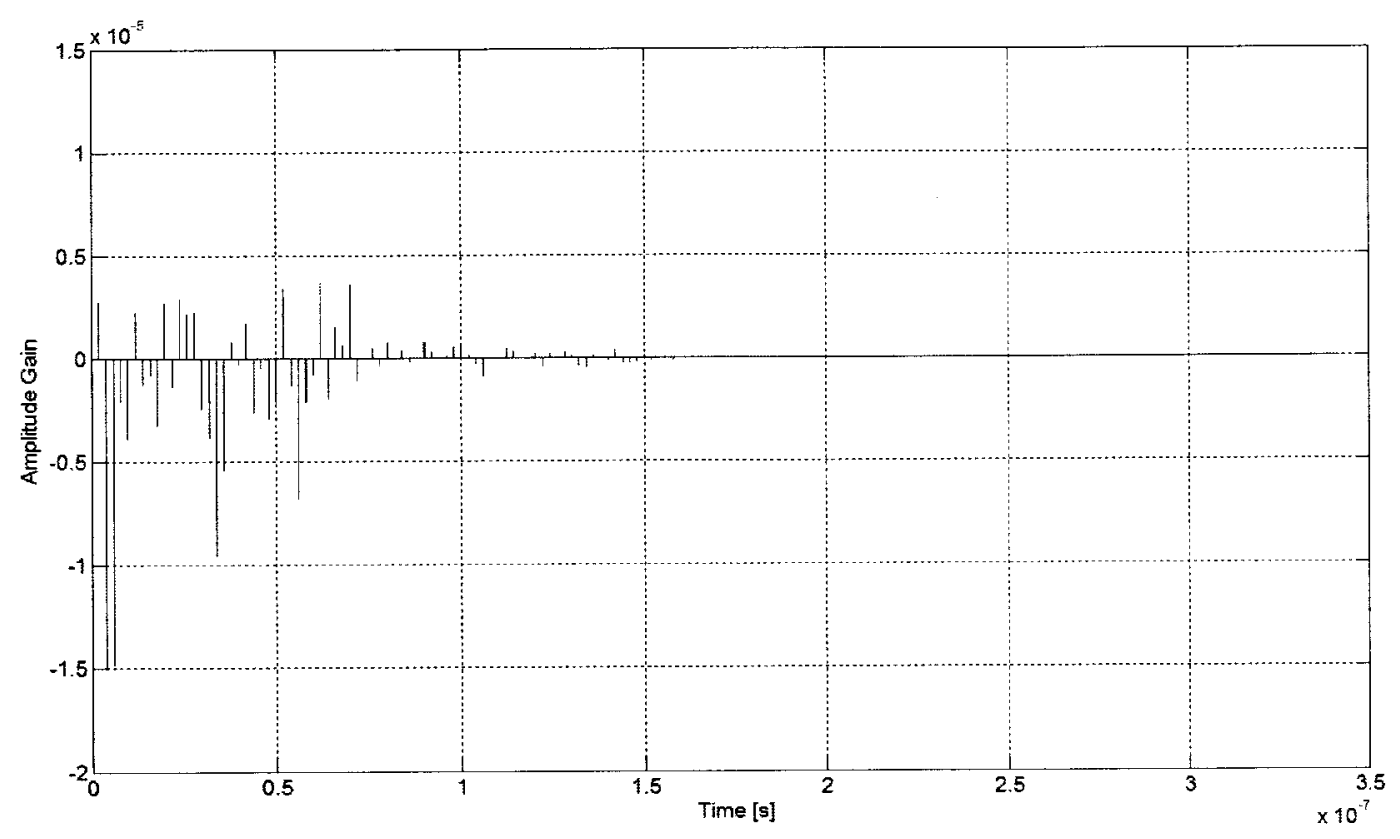

(a) $\mathrm{CM} 3$

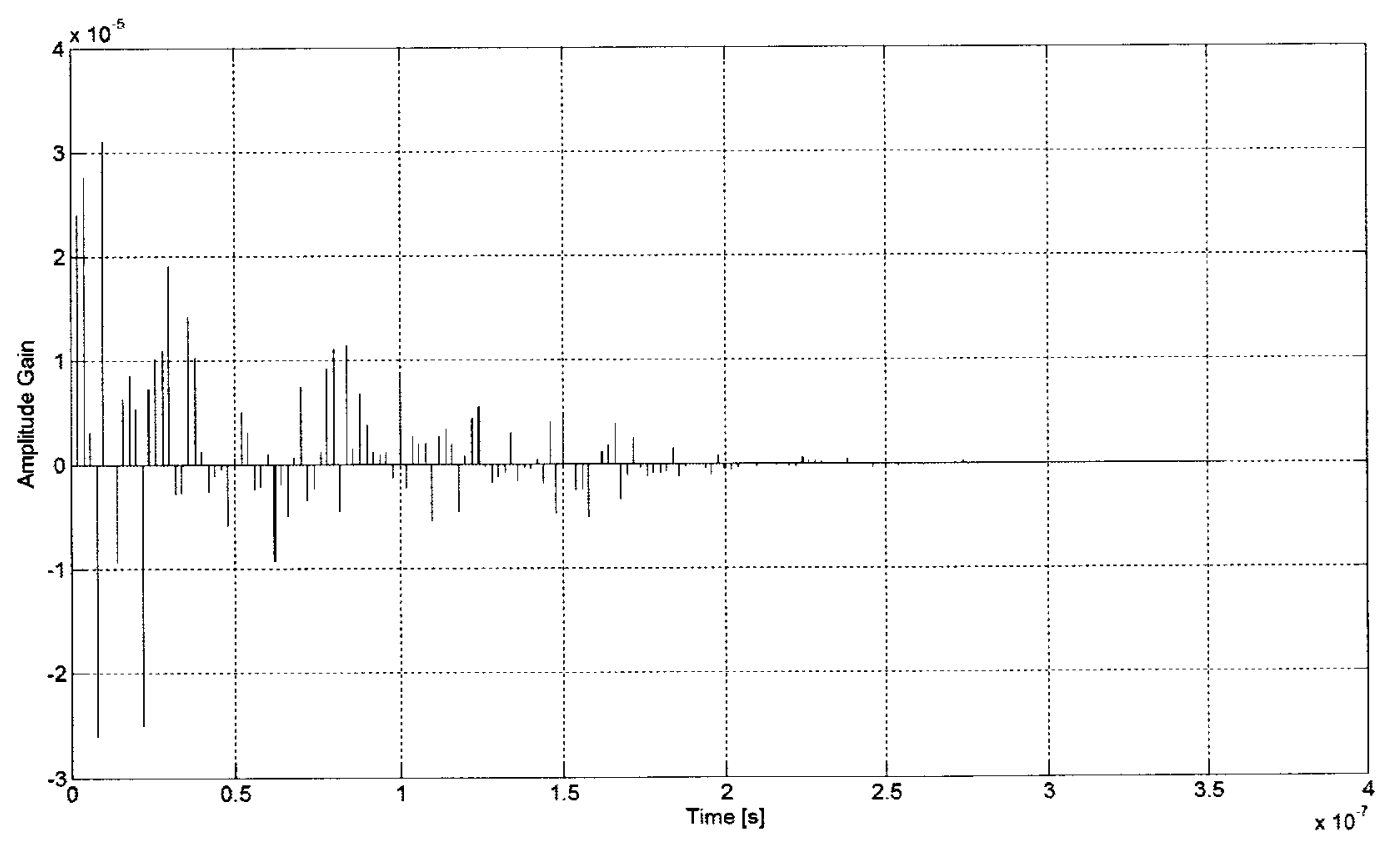

(b) CM4

Figure 4.7: A realization of the discrete time impulse response for the CM3 and the CM4 channel models. 


\subsection{Simulation of the Standard IEEE802.11a WLAN System}

\subsubsection{Transmitter}

The simulation blocks of the standard IEEE802.11a WLAN transmission scheme is depicted in figure 4.8 [10], where it can be summarized as follows:

\section{- Data Generator block}

A random binary serial data is generated with length equal to $N_{c a} \cdot n s \cdot k_{m}$, where $N_{c a}$ is the number of NBI sub-carriers, $k_{m}$ is the modulation level, $n s$ is the number of symbols transmitted per packet in one loop.

\section{- Serial to parallel Converter}

The input serial data is converted into a parallel data vectors according to the number of used sub-channels.

\section{- Modulation}

The standard IEEE802.11a WLAN uses many modulation schemes like BPSK, QPSK, 16QAM, and 64QAM. In our simulation experiments we used a coherent QPSK modulation scheme.

\section{- Data Mapping and IFFT Circuit}

According to the IEEE802.11a system standards, a data mapping technique is used in order to leave a group of frequencies at the lower and upper parts of the available bandwidth without modulation, so that avoid adjacent channel interference. The data mapping technique is depicted in figure 4.9 .

Assuming a 64-point IFFT is used, it can be seen that sub-carrier 0 and sub-carriers 27-37 are left unmodulated "Null value". The coefficients 1 to 26 are mapped to the same numbered IFFT inputs, while the coefficients 26 to 1 are copied into IFFT inputs 38 to 63 .

\section{- Guard Interval Insertion}

After performing an IFFT, the output is cyclically extended to the desired length. This is done with the aid of a guard interval (GI) insertion circuit 
to eliminate the impact of ISI caused by multi-path fading. The duration of the GI is $800 \mathrm{~ns}$, with a sampling rate equals to $20 \mathrm{Msamples} / \mathrm{s}$. The last 16 samples of each symbol ${ }^{3}$ is inserted in the front. The frame format is depicted in figure 4.10 .

\section{- Pilot Symbol Aided Modulation}

In order to compensate the effect of multi-path fading and the fluctuation of the amplitude and phase, pilot symbols are inserted at fixed time intervals. These pilot symbols are used to estimate the channel characteristics. The pilot carriers will be inserted in all frequencies at a known time period ${ }^{4}$ to accommodate for the independence of fading fluctuation.

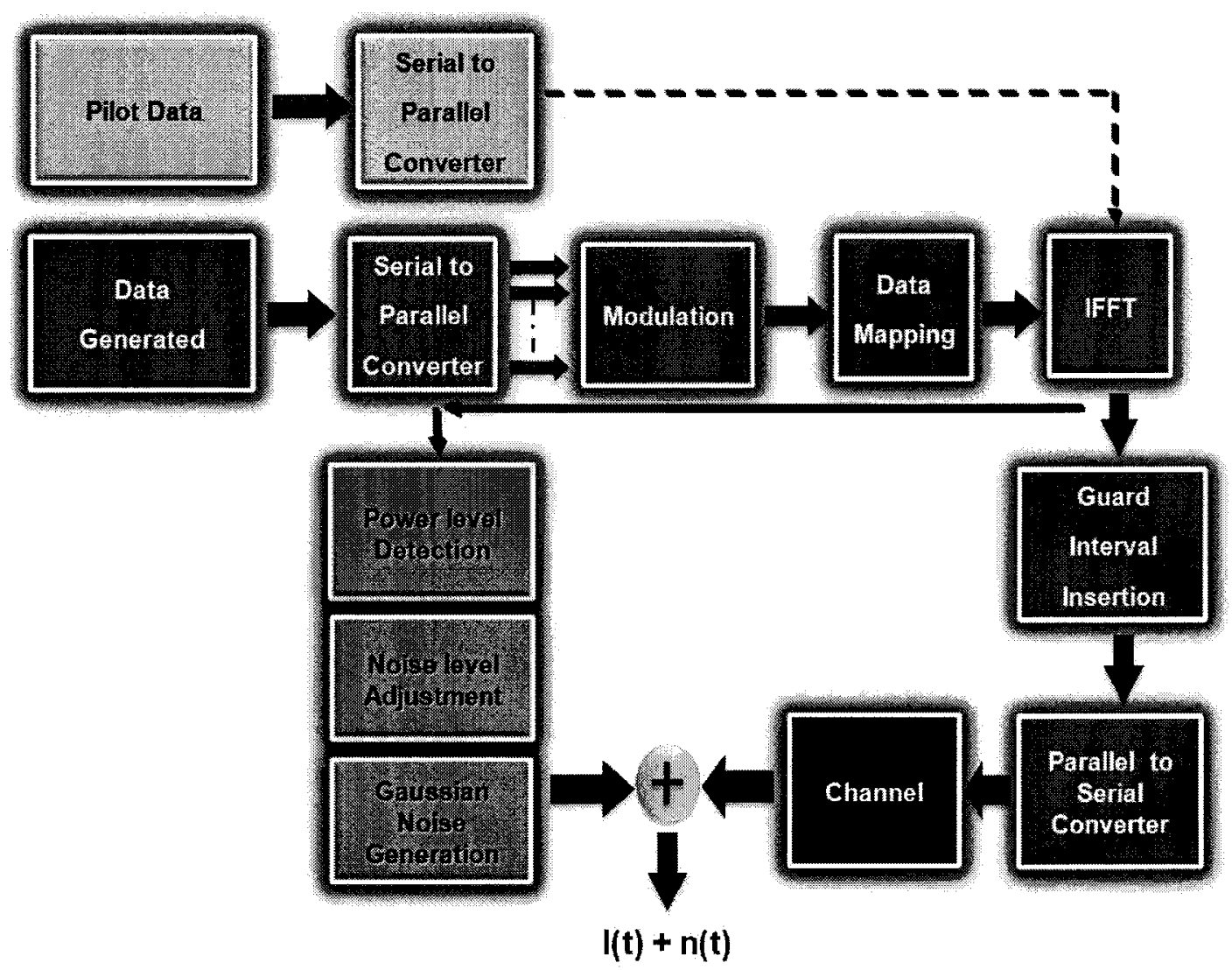

Figure 4.8: Simulation blocks of the NBI IEEE802.11a transmission scheme [10].

\footnotetext{
${ }^{3}$ The length of the guard interval is 16 samples.

${ }^{4}$ This technique of pilot insertion is called a "Block-type pilot arrangement".
} 


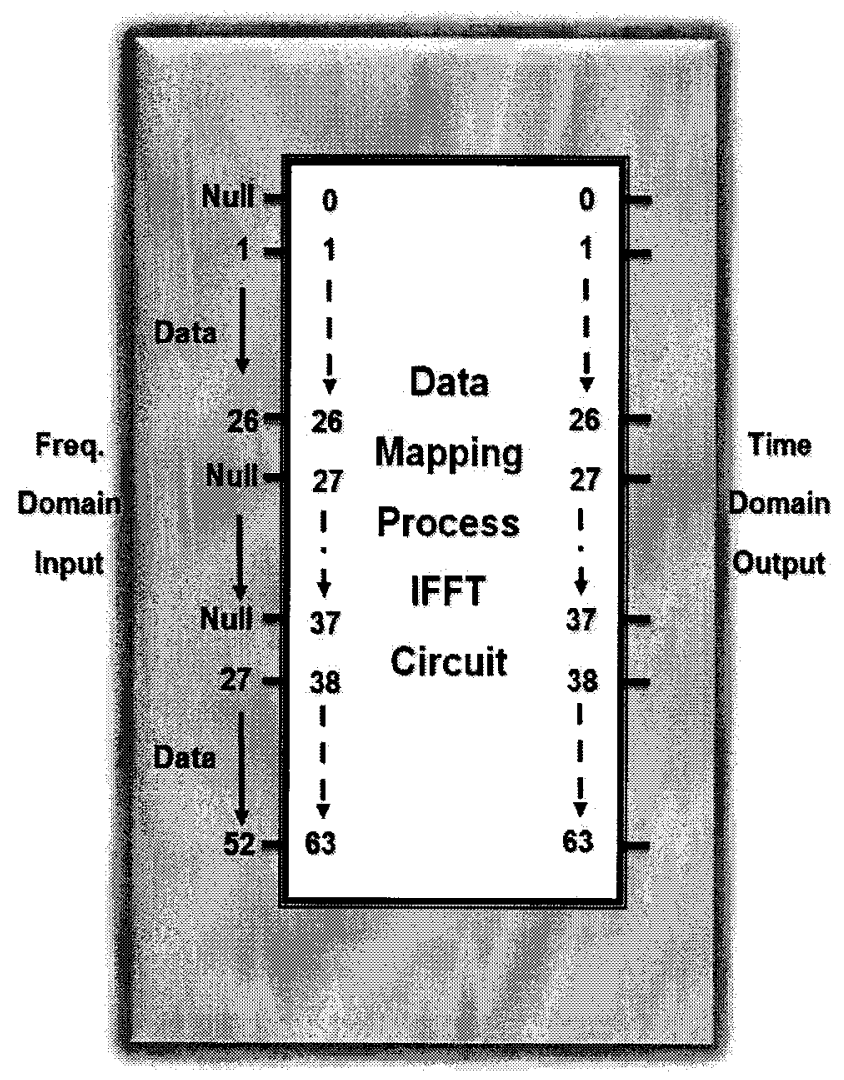

Figure 4.9: Data Mapping scheme.

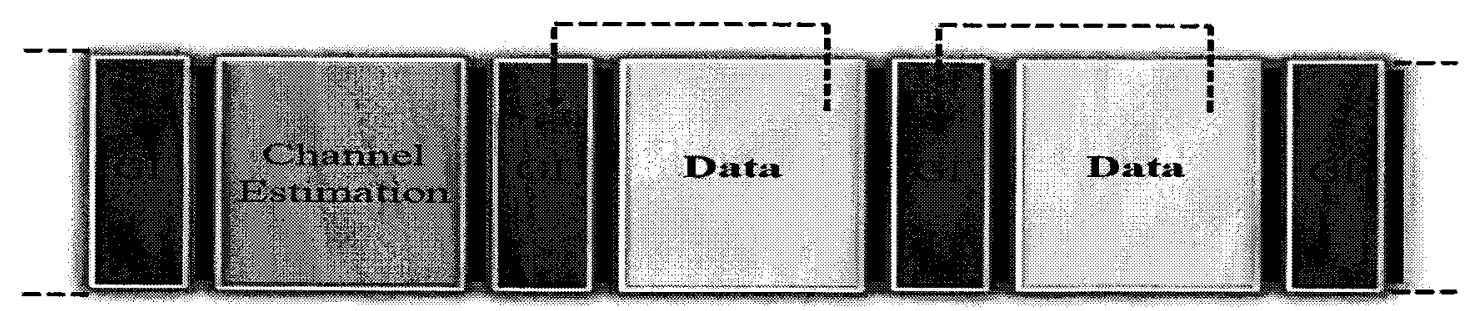

Figure 4.10: Frame format of the simulated WLAN IEEE802.11a system. 


\subsubsection{Receiver}

The receiver block diagram of the IEEE802.11a system is depicted in figure 4.11, where the incoming received data will initially pass through a GI removal block in order to remove the GI. After that the signal will be serial to parallel converted on the frequency axis in which we will get 64 sub-channels for 64-points FFT.

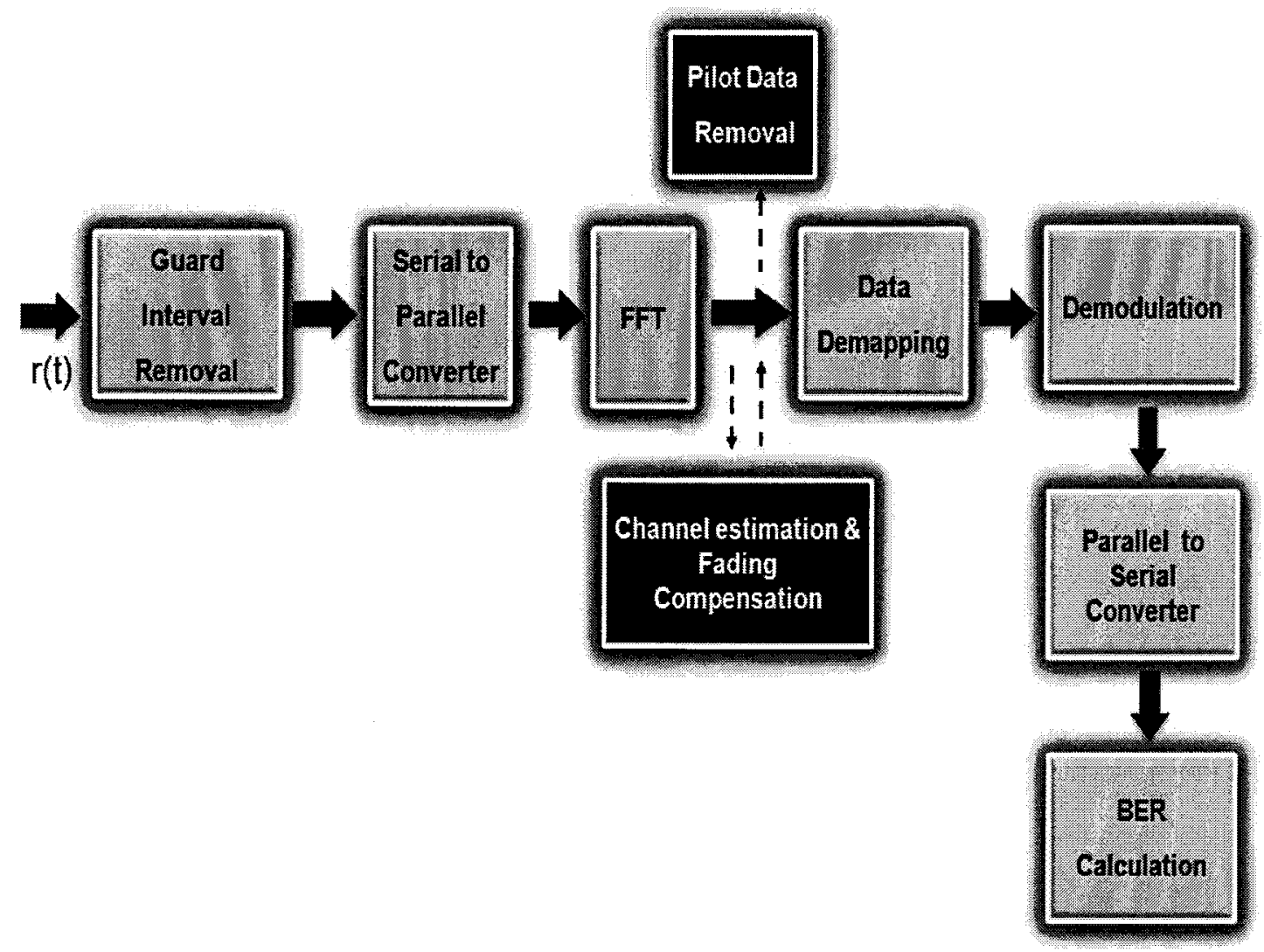

Figure 4.11: Reception Scheme of the WLAN IEEE802.11a narrow band system [10]

In a fading channel environment, channel estimation must be accomplished to compensate the effect of the channel on the incoming received signal. In case of perfect channel estimation, a de-mapping process is held on the data, followed by a demodulation process. The demodulated data will be parallel to serial converted before the BER performance is determined.

Table 4.2 shows the used parameters value of the simulated IEEE802.11a NBI signal. 
Table 4.2: The used IEEE802.11a simulated parameters value.

\begin{tabular}{|c|c|c|}
\hline Parameter & Symbol & Value \\
\hline \hline Symbol duration & $T_{s}$ & $4 \mu \mathrm{s}$ \\
\hline Number of parallel channels & $N_{c a}$ & 52 \\
\hline Symbol rate & $s_{r}$ & $250 \mathrm{kHz}$ \\
\hline sub-carriers spacing frequency & $\Delta \mathrm{f}$ & $312.5 \mathrm{kHz}$ \\
\hline Guard interval duration & $\mathrm{GI}$ & $800 \mathrm{~ns}$ \\
\hline Sampling rate & $\mathrm{f}_{s n}$ & $20 \mathrm{MHz}$ \\
\hline FFT length & & 64 \\
\hline
\end{tabular}

Figure 4.12 depicts a comparison between the simulated and analytical performances of the IEEE802.11a system in AWGN channel environment. The confidence interval of the simulated results at each point is shown.

The difference between the simulated curve and the analytic one results from the power lost due to the GI removal process, which can be calculated as:

$$
\text { shift }=-10 \log _{10}\left(1-\frac{\text { guard interval length }}{\text { symbol length }}\right)
$$

This shift equals to $0.969 \mathrm{~dB}$ according to the values associated for both the GI and symbol intervals.

\subsubsection{Simulation of the NBI Channel}

The NBI channel has been modeled as a frequency selective Rayleigh fading channel, where the mathematical model of the channel can be presented as follows [11]:

The field can be represented as a superposition of plane waves:

$$
E(t)=\operatorname{Re}\left[T(t) \exp \left(i \omega_{c} t\right)\right]
$$

where

$$
T(t)=E_{o} \sum_{n=1}^{N} c_{n} \exp \left[i\left(\omega_{m} t \cos \alpha_{n}+\phi_{n}\right)\right]
$$




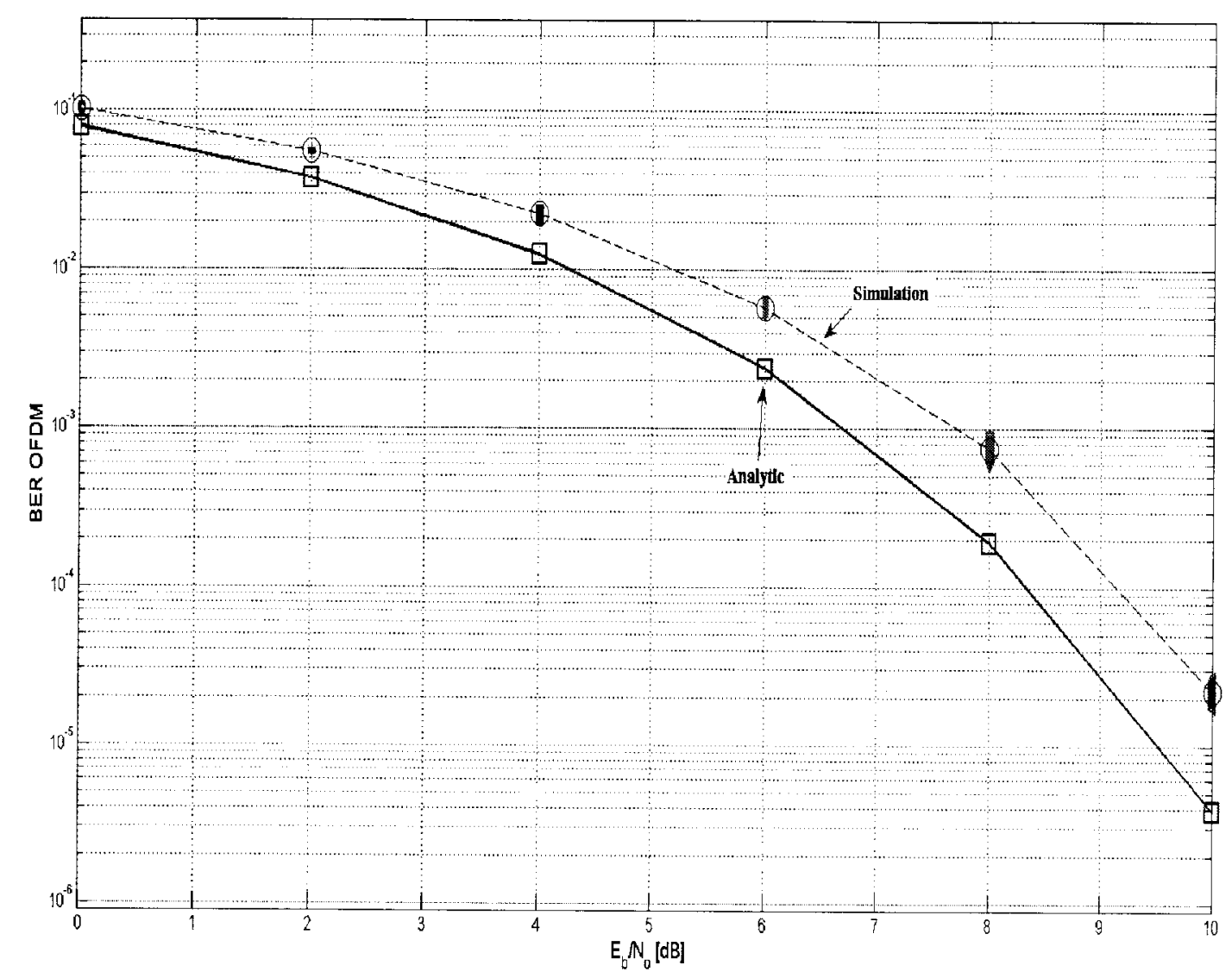

Figure 4.12: Comparison between the simulated and analytical performances of the IEEE802.11a system in AWGN channel, showing the confidence interval for the simulated results.

where $\omega_{c}$ is the carrier frequency of the transmitted signal, $N$ is the number of plane waves, $\omega_{m}$ is the maximum doppler shift and $E_{o} c_{n}$ is the real amplitude of the $n^{\text {th }}$ wave.

For a uniformly distributed arrival angles, $c_{n}^{2}=\frac{1}{N}$ and $\alpha_{n}=\frac{2 \pi n}{N}, \mathrm{n}=1,2, \ldots, \mathrm{N}$.

Let $\mathrm{N} / 2$ be an odd integer, the signal can be represented as [11]

$$
\begin{aligned}
T(t)=\frac{E_{o}}{\sqrt{N}}\left\{\sqrt{2} \sum_{n=1}^{N_{o}}\left[e^{i\left(\omega_{m} t \cos \alpha_{n}+\phi_{n}\right)}+e^{-i\left(\omega_{m} t \cos \alpha_{n}+\phi_{-n}\right)}\right]+\right. \\
\left.e^{i\left(\omega_{m} t+\phi_{N}\right)}+e^{-i\left(\omega_{m} t+\phi_{-N}\right)}\right\}, N_{o}=\frac{1}{2}\left(\frac{N}{2}-1\right)
\end{aligned}
$$


The factor $\sqrt{2}$ has been used so that the total power in $\mathrm{E}(\mathrm{t})$ will be unchanged. If $\mathrm{N}$ is large enough we may invoke the central limit theorem to conclude that $\mathrm{T}(\mathrm{t})$ is approximated as a complex Gaussian process and $|T|$ is Rayleigh as desired. As stated in [11], the Rayleigh approximation is quite good for $\mathrm{N} \geq 6$.

Figure 4.13 depicts the realization scheme of the previously mentioned mathematical model. $N_{o}$ low frequency oscillators with frequencies equals to the doppler shifts $\omega_{m} \cos \left(\frac{2 \pi n}{N}\right), \mathrm{n}=1,2, \ldots, N_{o}$ plus one with frequency $\omega_{m}$ are used to generate signals that are frequency shifted from the carrier frequency $\omega_{c}$ using modulation methods.

Note that, the phases $\beta_{n}$ are chosen appropriately so that the probability distribution of the resultant phase is as close as possible to a uniform distribution, $\frac{1}{2 \pi}$. Thus, $\beta_{n}=\frac{\pi n}{N}$, and $\alpha=\frac{\pi}{4}$.

$C_{I}(t)$ and $C_{Q}(t)$ are the in-phase and quadrature components respectively, which are approximately Gaussian random processes.

The Rayleigh distributed output faded signal can be written as

$$
y(t)=C_{I}(t) \cos \left(\omega_{c} t\right)+C_{Q}(t) \sin \left(\omega_{c} t\right) .
$$

\subsection{Simulation of the ICS}

Figure 4.14 depicts the simulation blocks of the canceller scheme. The simulation steps can be summarized as follows:

1. The NBI signal is generated ${ }^{5}$, interpolated ${ }^{6}$ and multiplied by the NBI channel fading coefficients.

2. The IR-UWB signal is generated ${ }^{7}$ and multiplied by the UWB channel fading coefficients.

3. The UWB signal power is measured by a power level detection block, where the noise power can be adjusted during the Gaussian noise generation

\footnotetext{
${ }^{5}$ As presented in figure 4.8 .

${ }^{6}$ According to the sampling rate of the UWB signal.

7 As presented in figure 4.1 or figure 4.2 according to the desired modulation type.
} 


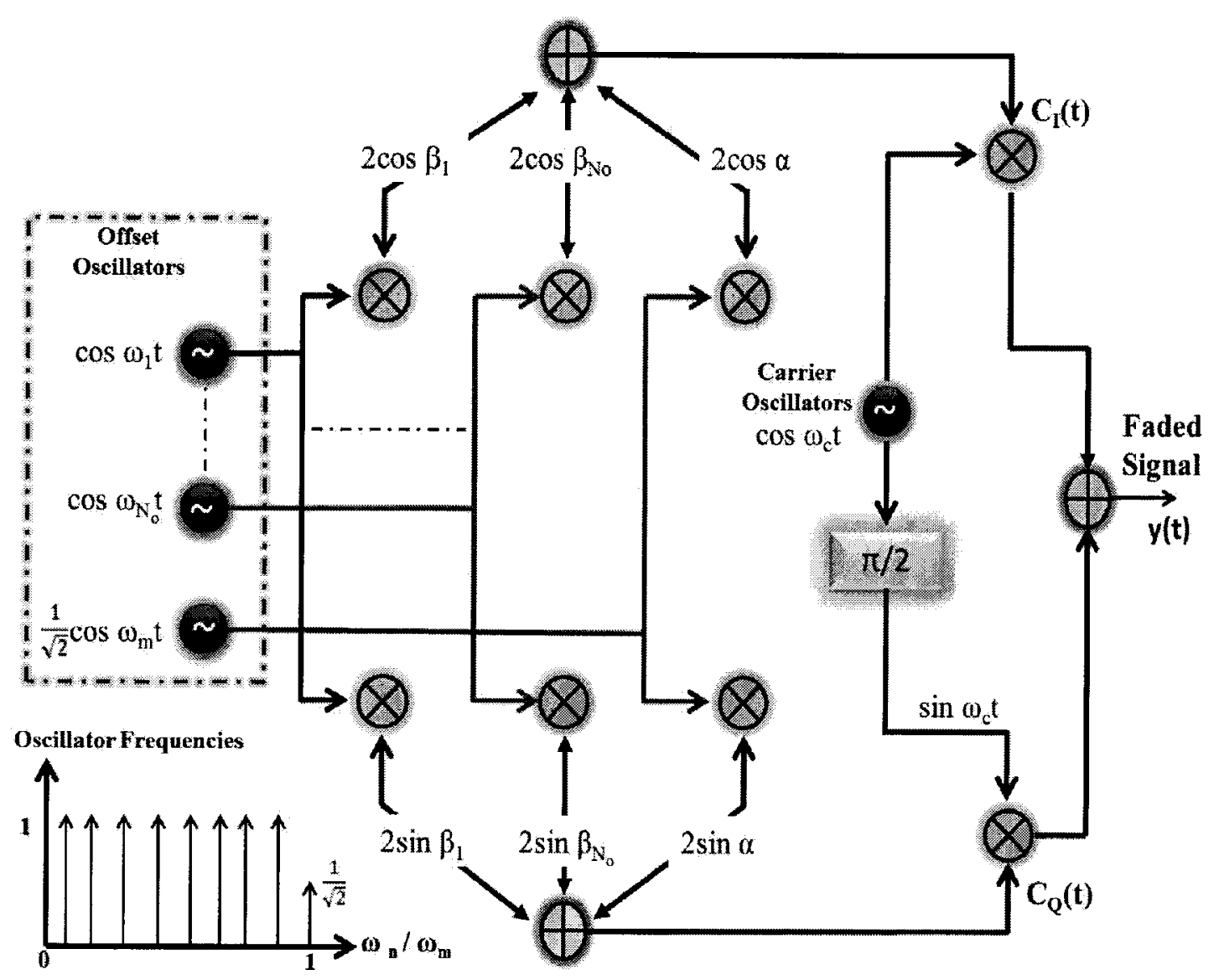

Figure 4.13: Realization scheme to simulate the Rayleigh distributed NBI fading channel [11]. 


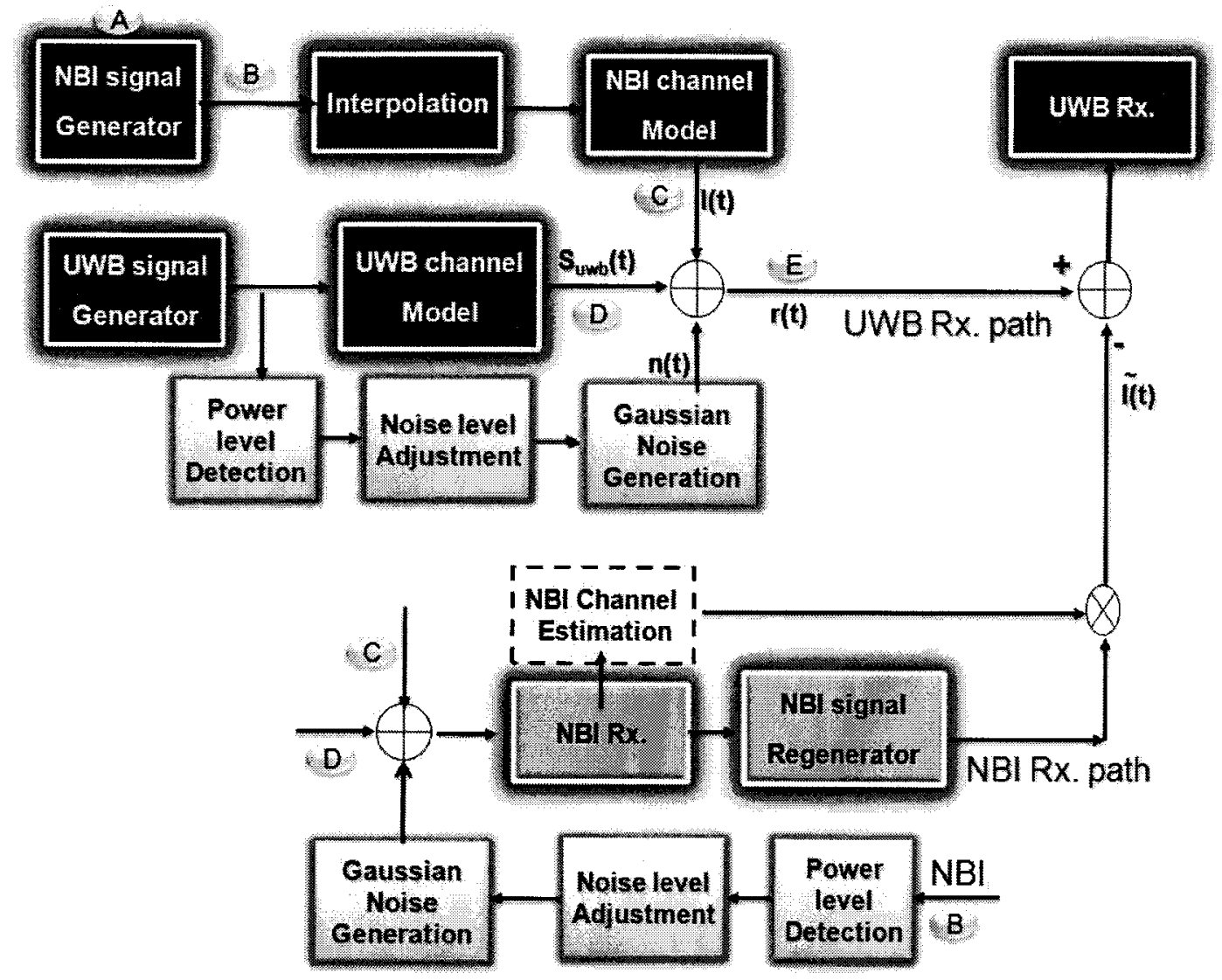

Figure 4.14: Simulation block diagram of the Canceller scheme.

process $^{8}$ according to the required SNR level.

4. In the NBI receiver path, the incoming received signal is intercepted by the NBI receiver ${ }^{9}$, so that the NBI signal can be demodulated.

5. The output from the demodulation process will be used to regenerate the NBI signal, which will be multiplied by the NBI channel estimate prior to its subtraction from the incoming received signal.

6. The output from the subtraction process will be passed through the UWB receiver, so that the transmitted bits can be estimated.

\footnotetext{
${ }^{8}$ The thermal noise generated in the UWB receiver.

${ }^{9}$ As presented in figure 4.11 .
} 


\section{Chapter 5}

\section{BER Analysis in Various UWB Channel Models}

In chapter (3), we noticed that the performance of the canceller scheme depends on the performance of a UWB system in the presence of NBI signals ${ }^{1}$. To investigate the performance of a UWB system in various channel models, using the ICS, we need to have analytic expressions for the BER performance of the UWB system in the presence of single or multiple tone interferers.

This chapter presents the analytical formulas, which were used later to evaluate the BER performance of the ICS under various fading channel models.

\subsection{BER Analysis of a UWB System in the Pres- ence of NBI in Log-normal Multi-path Fading Channels}

In this section, an approximate analytical closed form expression for the BER performance of a TH-PPM system in the presence of NBI signal in Log-normal multi-path fading channels is evaluated [74].

A binary communication system in a single user case is considered. For a matched filter reception, the transmitted desired signal of a TH-PPM can be written in the

\footnotetext{
${ }^{1}$ As presented in equation (3.5), where the NBI signal is modeled as multiple tone interferers.
} 
form $^{2}[16]$

$$
S_{P P M}(t)=\sqrt{E_{b}} \sum_{j=-\infty}^{\infty} p\left(t-j T_{f}-c_{j} T_{c}-\delta d_{\left\lfloor j / N_{s}\right\rfloor}\right)
$$

The narrowband interferer $I(t)$ is assumed to be a single tone signal. $I(t)$ can be written as

$$
I(t)=\sqrt{2 I} \cos \left(2 \pi f_{i} t+\phi_{i}\right)
$$

where $f_{i}$ is the interferer frequency, $\phi_{i}$ is a random variable (R.V.) considered to be uniformly distributed over the interval $[0,2 \pi)$ and $I$ is the transmitted power of the interferer.

The impulse response of the multi-path channel for the UWB signal is modeled as:

$$
h_{s}(t)=\sum_{\ell=0}^{L} a_{\ell} \delta\left(t-\tau_{\ell}\right)
$$

where $\mathrm{L}$ is the number of paths, $a_{\ell}$ is the multi-path fading coefficient of the $\ell^{\text {th }}$ path, $\delta($.$) is the Kronecker Delta and \tau_{\ell}$ is the delay corresponding to the $\ell^{\text {th }}$ path.

The channel fading coefficients $a_{\ell}$ can be defined as [2]

$$
a_{\ell}=p_{\ell} \beta_{\ell}
$$

where $p_{\ell}$ is a discrete R.V., $p_{\ell} \in\{-1,+1\}$ with equal probability to account for pulse inversion due to reflection and $\beta_{\ell}$ is the fading associated with the $\ell^{\text {th }}$ path.

For the narrowband interferer the channel impulse response can be written as

$$
h_{i}(t)=\alpha_{I} \delta\left(t-\tau_{i}\right)
$$

where $\alpha_{I}$ is the Rayleigh distributed channel gain and $\tau_{i}$ is the corresponding time delay.

\footnotetext{
${ }^{2}$ Parameters of this equation are previously presented in equation (2.12).
} 
The overall received signal $r(t)$ can be written as

$$
\begin{aligned}
r(t)=\sum_{\ell=0}^{L} \sum_{j=-\infty}^{\infty} a_{\ell} \cdot \sqrt{E_{b}} \cdot p\left(t-\tau_{\ell}\right. & \left.-j T_{f}-c_{j} T_{c}-\delta d_{\left\lfloor j / N_{s}\right\rfloor}\right) \\
& +\alpha_{I} \cdot \sqrt{2 I} \cdot \cos \left(2 \pi f_{i}\left(t-\tau_{i}\right)+\phi_{i}\right)+n(t)
\end{aligned}
$$

where $\mathbf{n}(\mathrm{t})$ is the AWGN signal with two sided power spectral density $N_{o} / 2$.

Without loss of generality, we will assume that the desired signal channel impulse response $h_{s}(t)$ is normalized such that $E\left[a_{s}^{2}\right]=1, E[$.$] denotes the expectation$ operator and $a_{s}^{2}=\sum_{\ell=0}^{L} a_{\ell}^{2}$.

Figure 5.1 depicts a schematic model for a UWB receiver with multiple tone interferers and additive noise and the associate fading channels for both the UWB and the NBI signals.

\subsubsection{Performance Evaluation}

It is assumed that the desired UWB signal undergoes Log-normal fading, whereas the NBI interferer signal is affected by Rayleigh fading. We also assume that the channel is perfectly known at the receiver.

The UWB correlation mask can be written as

$$
m^{\prime}(t)=\sum_{\ell=0}^{L} a_{\ell} \cdot m(t)
$$

where $\mathrm{m}(\mathrm{t})$ is given by

$$
m(t)=p\left(t-j T_{f}-\tau_{\ell}-c_{j} T_{c}\right)-p\left(t-j T_{f}-\tau_{\ell}-c_{j} T_{c}-\delta\right)
$$

The Rake receiver is simply matched to the received signal so its transfer function can be written as suggested in [71]

$$
|H(f)|=|M(f)| \cdot\left|H_{s}(f, \mathbf{a}, \mathbf{t})\right|
$$




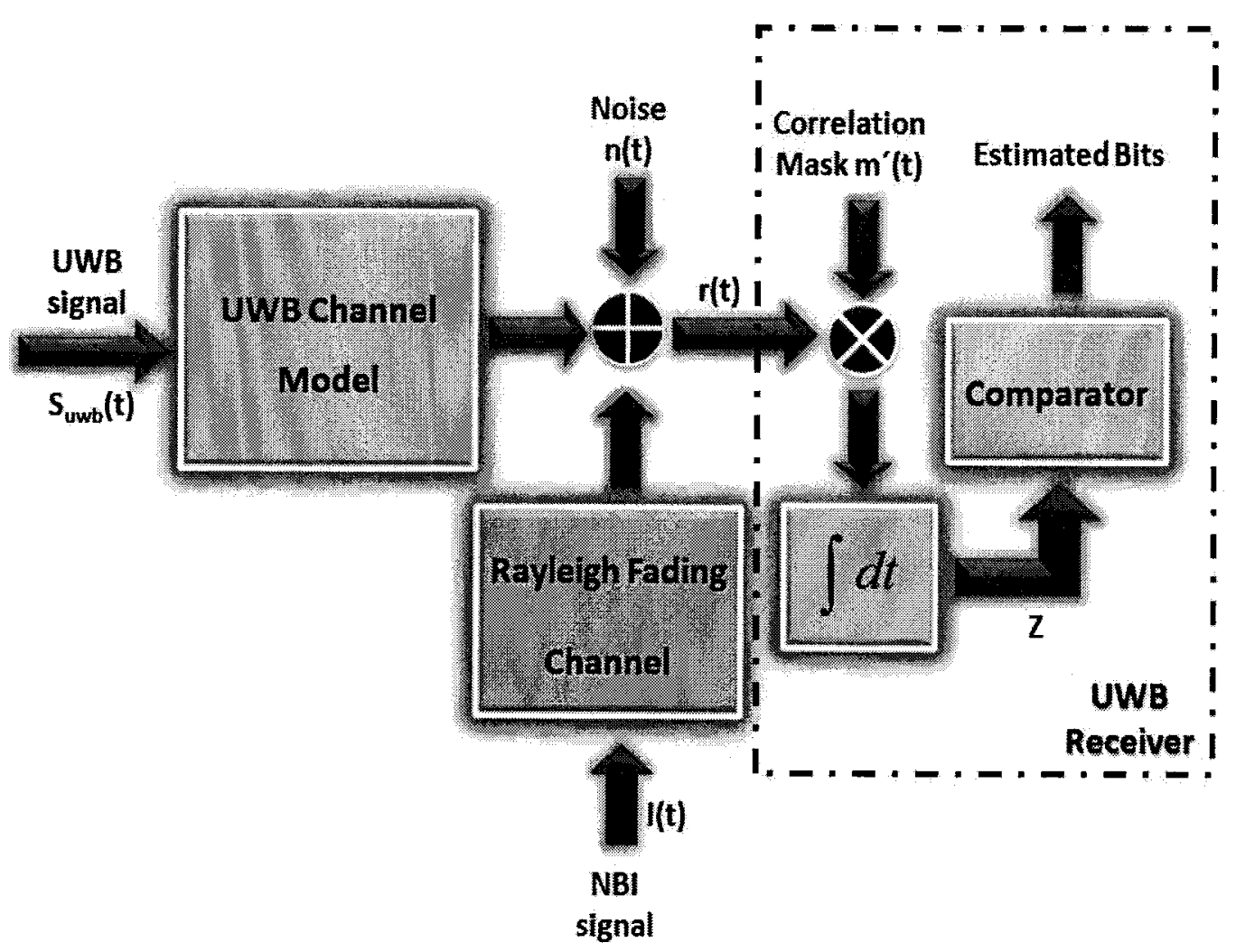

Figure 5.1: Schematic model for a UWB receiver system with a single tone interferer, additive noise and the associate fading channels. 
Where $M(f)$ represents the Fourier Transform of $m(t)$. For a TH-PPM UWB system, $M(f)$, is given by equation (3.10).

The second term $H_{s}(f, \mathbf{a}, \mathbf{t})$ is the Fourier Transform of the UWB signal channel impulse response $h_{s}(t)$ which can be written as

$$
\left|H_{s}(f, \mathbf{a}, \mathbf{t})\right|=\sum_{\ell=0}^{L} a_{\ell} \cdot \exp \left(-j 2 \pi f \tau_{\ell}\right)
$$

where $\mathbf{a}$ and $\mathbf{t}$ are random vectors representing the path gains and its corresponding time delays respectively.

Under the assumption of perfect synchronization, the decision statistic can be written as

$$
\begin{gathered}
Z=\sum_{j=-\infty}^{\infty} \int_{j T_{f}}^{j\left(T_{f}+1\right)} r(t) \cdot m(t) d t \\
Z=S_{p p m}+I_{N}+n
\end{gathered}
$$

where $S_{p p m}, I_{N}$ and $n$ are the desired signal, interference and noise components respectively.

$$
S_{p p m}=\sqrt{E_{b}}(1-\varrho) a_{s}^{2}
$$

The interference term $I_{N}$ can be written as

$$
I_{N}=\alpha_{I} \sqrt{2 I} \cdot\left|M\left(f_{i}\right)\right| \cdot\left|H_{s}\left(f_{i}, \mathbf{a}, \mathbf{t}\right)\right| \cos \left(\phi_{i}\right)
$$

The phase term $\left[\arg \left[H\left(f_{i}\right)\right]+2 \pi f_{i}\left(t-\tau_{i}\right)\right]$ is included within the random phase $\phi_{i}$, conditioned on the R.V. $\left[\left|M\left(f_{i}\right)\right| \cdot\left|H_{s}\left(f_{i}, \mathbf{a}, \mathbf{t}\right)\right|\right]$. $I_{N}$ can be considered conditionally Gaussian with variance $\left[I \cdot\left|M\left(f_{i}\right)\right|^{2} \cdot\left|H_{s}\left(f_{i}, \mathbf{a}, \mathbf{t}\right)\right|^{2}\right]$.

The variance of the noise can be written as

$$
\sigma_{N}^{2}=N_{o}(1-\varrho) a_{s}^{2}
$$

The total disturbance due to the NBI plus noise is also conditionally Gaussian with variance

$$
\sigma_{\text {tot }}^{2}=I \cdot\left|M\left(f_{i}\right)\right|^{2} \cdot\left|H_{s}\left(f_{i}, \mathbf{a}, \mathbf{t}\right)\right|^{2}+\sigma_{N}^{2}
$$


The conditional BER, conditioned on $(\mathbf{a}, \mathbf{t})$ can be written as

$$
P_{e \mid \mathbf{a}, \mathbf{t}}=Q(\sqrt{\gamma(\mathbf{a}, \mathbf{t})})
$$

where

$$
\gamma(\mathbf{a}, \mathbf{t})=\frac{a_{s}^{2}}{\frac{N_{o}}{E_{b}(1-\varrho)}+\frac{\left|H\left(f_{i}\right)\right|^{2}}{S I R \cdot T_{b} \cdot(1-\varrho)^{2} \cdot a_{s}^{2}}}
$$

In order to evaluate the BER, we will initially average over the random time delays $\mathbf{t}$ for a fixed path gains $\mathbf{a}$ and then we average over the path gains $\mathbf{a}$. We will define a new R.V. $\chi=\left|H_{s}\left(f_{i}, \mathbf{a}, t\right)\right|^{2}$ that depends on the random vector $\mathbf{t}$ with fixed path gains a.

The conditional BER can be rewritten as

$$
P_{e \mid \mathbf{a}}=\xi(\chi)=Q(\sqrt{\gamma(\chi)})
$$

where

$$
\gamma(\chi)=\frac{a_{s}^{2}}{\frac{N_{o}}{E_{b}(1-\varrho)}+\frac{\left|M\left(f_{i}\right)\right|^{2} \cdot \chi}{S I R \cdot T_{b} \cdot(1-\varrho)^{2} \cdot a_{s}^{2}}}
$$

As presented in [75] this expectation can be averaged over the R.V. $\chi$ by means of "Perturbation Theory" to find an approximation to this expectation without the need of integration (Stirling formula). This expansion is done in central differences up to the third order to have

$$
\begin{aligned}
& P_{e} \mid \mathbf{a} \simeq \xi(\mu)+\frac{\sigma^{2}}{2} \frac{\xi(\mu+h)-2 \xi(\mu)+\xi(\mu-h)}{h^{2}} \\
&+\frac{v}{12} \frac{\xi(\mu+2 h)-2 \xi(\mu+h)+2 \xi(\mu-h)-\xi(\mu-2 h)}{h^{3}}
\end{aligned}
$$

where

$$
\begin{aligned}
& \mu(\mathbf{a})=E_{\mathbf{t}}\left[\left|H_{s}\left(f_{i}, \mathbf{a}, \mathbf{t}\right)\right|^{2}\right] \\
& \sigma^{2}(\mathbf{a})=E_{\mathrm{t}}\left[\left|H_{s}\left(f_{i}, \mathbf{a}, \mathbf{t}\right)\right|^{2}-\mu(\mathbf{a})\right]^{2} \\
& v(\mathbf{a})=E_{\mathrm{t}}\left[\left|H_{s}\left(f_{i}, \mathbf{a}, \mathbf{t}\right)\right|^{2}-\mu(\mathbf{a})\right]^{3}
\end{aligned}
$$

Equation (5.22) represents the first, second and third central moments respectively. 
As shown in [71] these moments can be approximated as follows

$$
\begin{aligned}
& \mu(\mathbf{a})=a_{s}^{2} \\
& \sigma^{2}(\mathbf{a})=(1-\varpi) \cdot a_{s}^{4} \\
& v(\mathbf{a})=(2-6 \varpi+4 y) \cdot a_{s}^{6}
\end{aligned}
$$

where $\varpi=\sum_{\ell=0}^{L} \Omega_{\ell}^{2}$ and $y=\sum_{\ell=0}^{L} \Omega_{\ell}^{3}$.

$\Omega_{\ell}$ is the normalized power delay profile of the channel. For an exponential power delay profile; $\Omega_{\ell}$ is given by [59]

$$
\Omega_{\ell}=E\left[a_{\ell}^{2}\right]=\frac{1-\exp \left(-\varepsilon^{\prime}\right)}{1-\exp \left(-L \varepsilon^{\prime}\right)} \exp \left[-(\ell-1) \varepsilon^{\prime}\right]
$$

where $\varepsilon^{\prime}$ is the decay rate.

Note that, this normalization of the power delay profile implies that the sum of the attenuation power of all paths is one $\left(\sum_{\ell=0}^{L} \Omega_{\ell}=1\right)$.

The conditional BER over a can be written as

$$
P_{e} \mid \mathbf{a} \simeq \sum_{n=1}^{N} b_{n} \cdot Q\left(\sqrt{\gamma\left(q_{n} a_{s}^{2}\right)}\right)
$$

where $\mathrm{N}$ is the number of terms in the expansion. For the third order expansion $(N=4)$. The weights $b_{n}$ and $q_{n}$ are:

$\mathbf{b}=\left(1 / 6+M^{\prime}, 2 / 3,1 / 6-2 M^{\prime}, M^{\prime}\right)$ and $\mathbf{q}=(0,1,1+\sqrt{3 P}, 1+2 \sqrt{3 P})$, $M^{\prime}=\frac{1-3 \varpi+2 y}{18 \sqrt{3}(1-\varpi)^{3 / 2}}$ and $(P=1-\varpi)$.

Now, equation (5.25) depends only on the term $\left(a_{s}^{2}=\sum_{\ell=0}^{L} a_{\ell}^{2}\right)$.

It has been reported in [76] that $a_{s}^{2}$ can be considered as a Log-normally distributed R.V.. By averaging equation (5.25) over $a_{s}^{2}$, the BER can be written as

$$
P_{e} \simeq \int_{0}^{\infty} \sum_{n=1}^{N} b_{n} \cdot Q\left(\sqrt{\gamma\left(q_{n} \cdot a_{s}^{2}\right)}\right) f\left(a_{s}\right) d a_{s}
$$


where $f\left(a_{s}\right)$ is the PDF of $a_{s}$, normally distributed with mean $\mu_{r}$ and variance $\sigma_{r}^{2}$. $f\left(a_{s}\right)$ can be written as

$$
f\left(a_{s}\right)=\frac{1}{a_{s} \sigma_{r} \sqrt{2 \pi}} \exp \left(\frac{-\left(\ln \left(a_{s}\right)-\mu_{r}\right)^{2}}{2 \sigma_{r}^{2}}\right)
$$

Parameters $\left(\mu_{k}, \sigma_{k}\right)$ are usually introduced to characterize a Log-normal distribution in wireless communications, where as presented in [76] $\mu_{k}=\lambda \mu_{r}, \sigma_{k}=\lambda \sigma_{r}$ and $\lambda=10 / \ln (10)$. The parameter $\sigma_{k}$, known as the $\mathrm{dB}$ spread, and its value ranges between 6 and $12 \mathrm{~dB}$ for most of the wireless communication systems [76], and between 2 and $5 \mathrm{~dB}$ for the UWB communication systems [32].

While exact closed-form expressions for the Log-normal sum PDF are unknown, several analytical approximation methods exist in the literature [77], [78], and [79].

A closed form approximation of the average probability of error over a Log-normal fading channel is presented in $[80]^{3}$. The Log-normally distributed random variable $a_{s}^{2}$ can be averaged by following the same approach suggested in [80].

Finally, the average BER performance of a UWB system in the presence of NBI can be evaluated as

$$
\begin{array}{r}
P_{e} \simeq \sum_{n=1}^{N} b_{n} \cdot \frac{\eta_{o}}{\sqrt{2 \pi} \sigma_{r}} \exp \left[-\frac{W_{o}\left(\gamma_{o} \cdot e^{\mu_{r}} \sigma_{r}^{2} / 2\right)}{\sigma_{r}^{2}}\left\{\eta_{1} \cdot\left[\frac{W_{o}\left(\gamma_{o} \cdot e^{\mu_{r}} \sigma_{r}^{2} / 2\right)}{\sigma_{r}^{2}}\right]^{\eta_{2}-1}+\right.\right. \\
\left.\left.\frac{W_{o}\left(\gamma_{o} \cdot e^{\mu_{r}} \sigma_{r}^{2} / 2\right)}{2}\right\}\right]
\end{array}
$$

where $W_{o}$ is the Lambert $\mathrm{W}$ function, $\mathrm{N}>1$ and $\eta_{o}, \eta_{1}$, and $\eta_{2}$ are parameters to adjust the tightness of the approximation.

The values of $\eta_{o}, \eta_{1}$, and $\eta_{2}$ are related to the dB-spread as follows:

For $\sigma_{k} \in[2,6)$, they are given by [80]:

$$
\left\{\begin{array}{l}
\eta_{o}=\left[0.06516\left(\sigma_{k}+24.47\right)\right]^{2}-2.5959 \\
\eta_{1}=-\left[0.06708\left(\sigma_{k}-18.7231\right)\right]^{2}+2.7904 \\
\eta_{2}=\left[0.03162\left(\sigma_{k}-17.001\right)\right]^{2}+0.662
\end{array}\right.
$$

\footnotetext{
${ }^{3}$ Presented in this paper in equation (31).
} 
and for $\sigma_{k} \in[6,12]$

$$
\left\{\begin{array}{l}
\eta_{o}=0.258\left(\sigma_{k}-6\right)+1.345 \\
\eta_{1}=-\left[0.0592\left(\sigma_{k}-21.5\right)\right]^{2}+2.9046 \\
\eta_{2}=\left[0.02646\left(\sigma_{k}-20.07\right)\right]^{2}+0.6434
\end{array}\right.
$$

and,

$$
\gamma_{o}=\left(\frac{N_{o}}{E_{b}(1-\varrho)}+\frac{\left|H\left(f_{i}\right)\right|^{2} \cdot q_{n}}{S I R \cdot T_{b} \cdot(1-\varrho)^{2}}\right)^{-1}
$$

\subsubsection{Analytical Validation}

The performance of a TH-PPM system in a frequency selective Log-normal fading channel has been evaluated using the analytic expression presented in equation (5.28). In order to validate our analytic evaluation, a comparison with the analytical results obtained in [71], which are based on a Nakagami-m distribution is accomplished.

This comparison is done on the basis that, if the two distributions can coincide with each other, the same result should be obtained from the analytical expression presented in [71] and our derived analytical expression.

As reported in [81] and [82], for high values of the Nakagami-m parameter, it is widely accepted that this distribution may be used to approximate the Log-normal distribution. Figures 5.2 and 5.3 show selections of the Nakagami $[N a k a g a m i(m, \Omega)]$ and the $\log$-normal $\left[\operatorname{Logn}\left(\mu_{r}, \sigma_{r}\right)\right] \mathrm{PDF}$, which illustrate this relationship for low $(\mathrm{m}=4,8$, and 10$)$ and high $(\mathrm{m}=20$, and 75$)$ Nakagami-m distribution fading parameter values along with their corresponding Log-normal parameters $\left(\mu_{r}, \sigma_{r}\right)$ values respectively.

It can be seen that for a value of the Nakagami fading parameter $m>20$, the two distributions are very similar. The validation of our analytic result will be achieved in a flat fading channel with the previously mentioned (low and high) values of the Nakagami-m fading parameter and their corresponding Log-normal parameters values. 


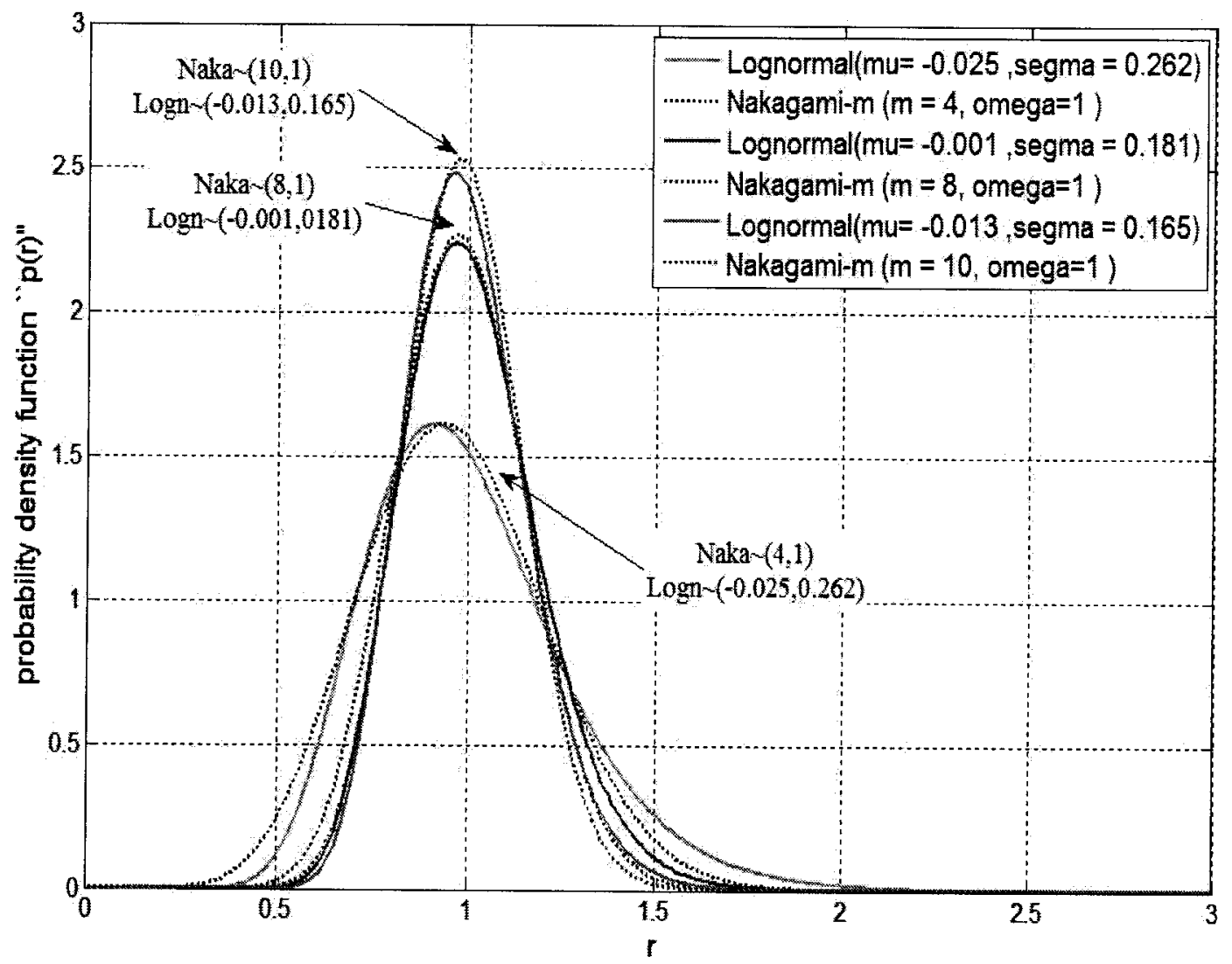

Figure 5.2: PDF relation between Nakagami-m and Log-normal distributions for Low values of $m$. 


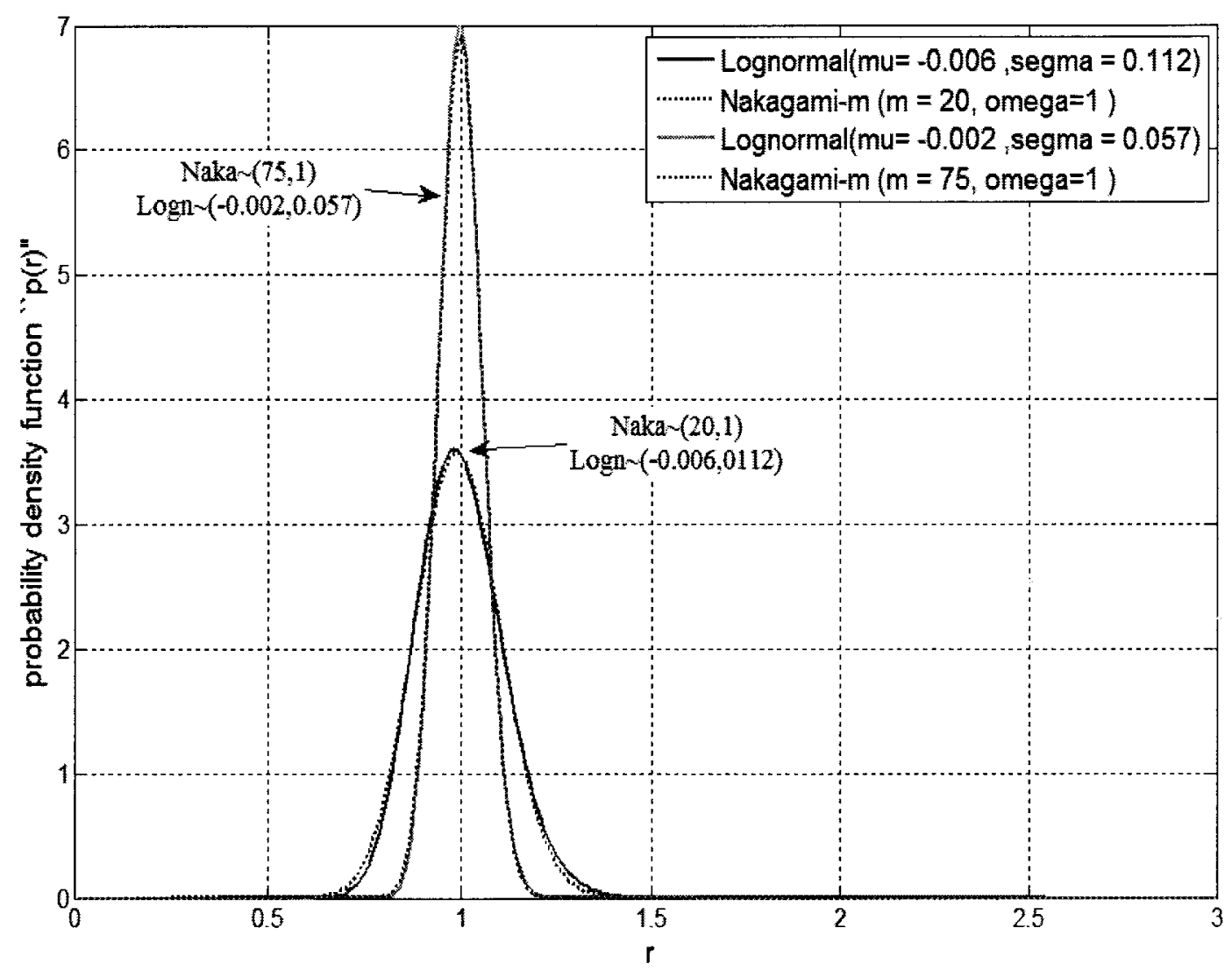

Figure 5.3: PDF relation between Nakagami-m and Log-normal distributions for high values of $\mathrm{m}$. 
For these values of the Log-normal parameters, the $\mathrm{dB}$ spread value is out of its practical range $[2,12]$ and the average BER presented in equation (5.28) will have to be modified in order to keep the tightness with the analytic expression presented in equation (5.26) as close as possible.

The average BER in a flat fading channel for the new $\mathrm{dB}$ spread values will be evaluated by following the same approach suggested in [80], where the values of the variables $\mathrm{N}, \mathrm{b}$ and $\mathrm{q}$ will be equal to one. It is given by

$$
\begin{array}{r}
P_{e} \simeq \frac{\eta_{o}}{\sqrt{2 \pi} \sigma_{r}} \cdot \exp \left[-\frac{W_{o}\left(2 \gamma_{o}^{\prime} \cdot e^{2 \mu_{r}} \cdot \sigma_{r}^{2}\right)}{4 \sigma_{r}^{2}} \cdot\left\{\eta_{1} \cdot\left[\frac{W_{o}\left(2 \gamma_{o}^{\prime} \cdot e^{2 \mu_{r}} \cdot \sigma_{r}^{2}\right)}{12 \sigma_{r}^{2}}\right]^{\eta_{2}-1}+\right.\right. \\
\left.\left.\frac{W_{o}\left(2 \gamma_{o}^{\prime} \cdot e^{2 \mu_{r}} \cdot \sigma_{r}^{2}\right)}{2}\right\}\right]
\end{array}
$$

where

$$
\gamma_{o}^{\prime}=\left(\frac{N_{o}}{E_{b}(1-\varrho)}+\frac{\left|M\left(f_{i}\right)\right|^{2}}{S I R \cdot T_{b} \cdot(1-\varrho)^{2}}\right)^{-1}
$$

In [71], the average BER performance for a UWB system in a Nakagami-m flat fading was given by:

$$
P_{e}=\frac{\Gamma\left(m+\frac{1}{2}\right)}{2 \sqrt{\pi} \Gamma(m+1)}\left(\frac{m}{\vartheta}\right)^{m}{ }_{2} F_{1}\left(m, m+\frac{1}{2} ; m+1 ;-\frac{m}{\vartheta}\right)
$$

where $\vartheta$ can be written as

$$
\vartheta=\left(\frac{N_{o}}{E_{b}(1-\varrho)}+\frac{\left|M\left(f_{i}\right)\right|^{2}}{S I R \cdot T_{b} \cdot(1-\varrho)^{2}}\right)^{-1}
$$

In the following figures, a second derivative Gaussian received pulse is used with values: $\tau_{p}=0.5 \mathrm{~ns}, N_{s}=2$ pulses/bit, a frame duration $T_{f}=100 \mathrm{~ns}$, a PPM time shift $\delta=0.3 \mathrm{~ns}$, the correlation parameter $\varrho=-0.587$, a $\mathrm{TH}$ code sequence $c_{o}=c_{1}=0$, the chip width $T_{c}=1.5 \mathrm{~ns}$, and finally, the interferer frequency $f_{i}=$ $2.412 \mathrm{GHz}$.

Figure 5.4 depicts the BER performance of a TH-PPM system in the presence of $\mathrm{NBI}, \mathrm{SIR}=-10 \mathrm{~dB}$ for different values of $\mathrm{m}$ evaluated by equation (5.32), as expected the performance is severely degraded by the presence of fading on the desired signal. 


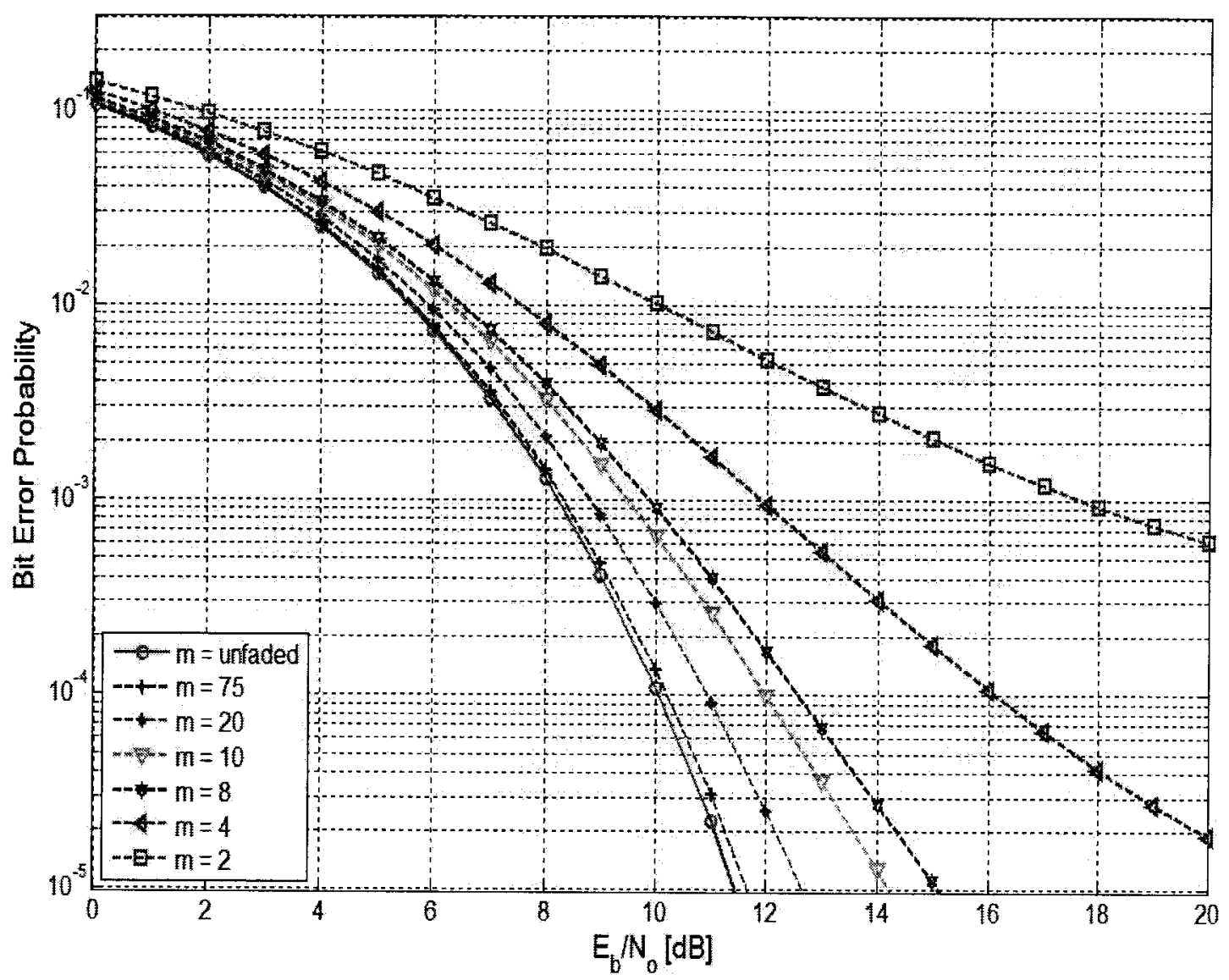

Figure 5.4: TH-PPM BER performance in a Nakagami-m flat fading channel for different values of $m$ and $S I R=-10 \mathrm{~dB}$.

Figures 5.5 and 5.6 depict this comparison in a flat fading channel in the presence of a single tone interferer at $\mathrm{SIR}=-10 \mathrm{~dB}$, for low and high values of the Nakagami-m parameter and their corresponding Log-normal parameters respectively.

It can be seen that, the two analytic formulas show similar behavior for high values of the Nakagami fading parameter $m$, whereas for the low-values the output results from the two analytic formulas differ.

The performance of the TH-PPM system for different SIRs is depicted in figure 5.7 for $(\mathrm{m}=75)$. It can be seen that, a good agrement is accomplished between the two analytic formulas. To this end, we can conclude the validation of our derived analytical formula. 


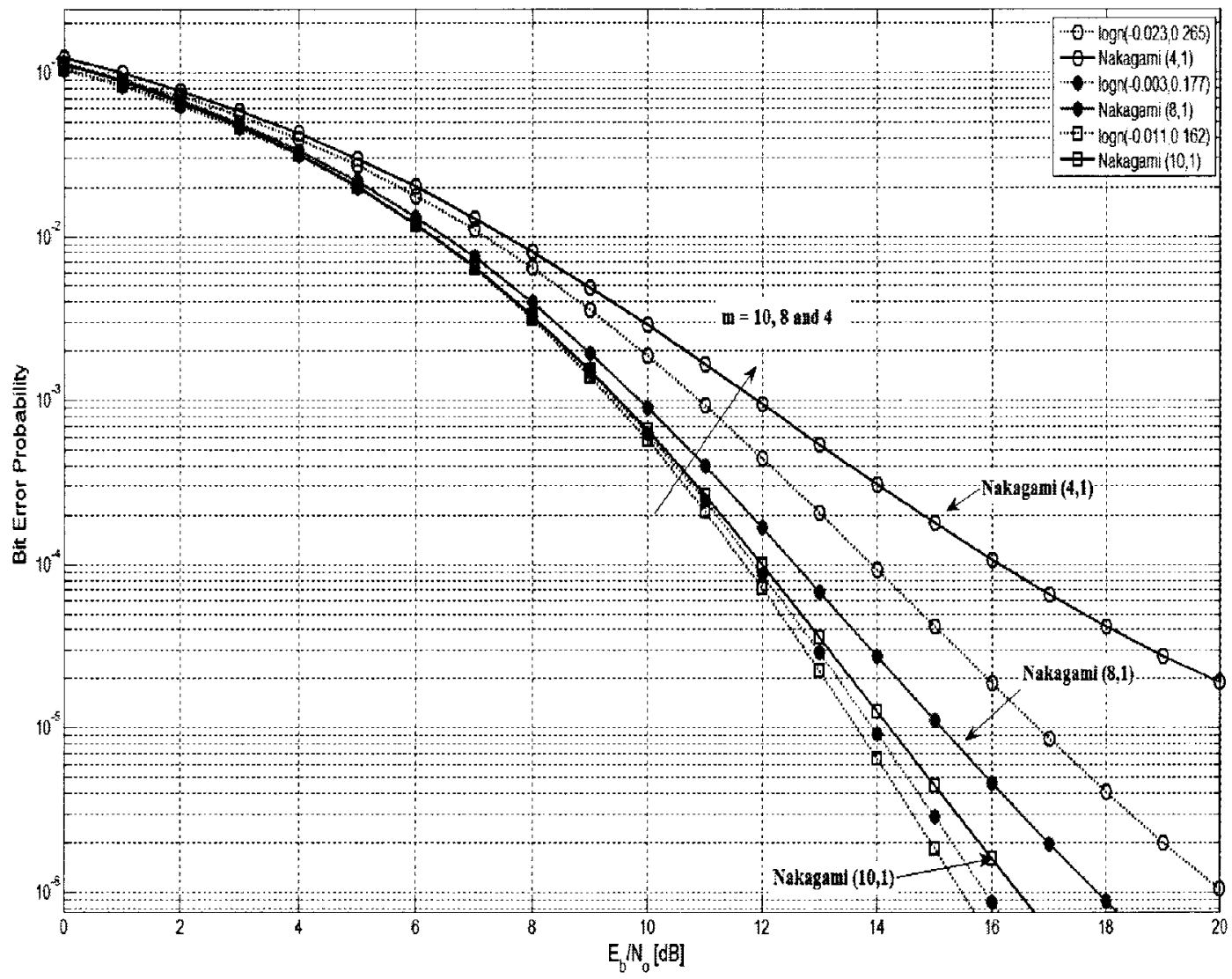

Figure 5.5: TH-PPM BER performance in the presence of a single tone interferer $(\mathrm{SIR}=-10 \mathrm{~dB})$, for $\left(\mathrm{m}=4, \eta_{o}=0.07, \eta_{1}=1.3569, \eta_{2}=0.9146\right),\left(\mathrm{m}=8, \eta_{o}=0.04\right.$, $\left.\eta_{1}=1.2927, \eta_{2}=0.9289\right)$, and $\left(\mathrm{m}=10, \eta_{o}=0.034, \eta_{1}=1.2914, \eta_{2}=0.9281\right)$. 


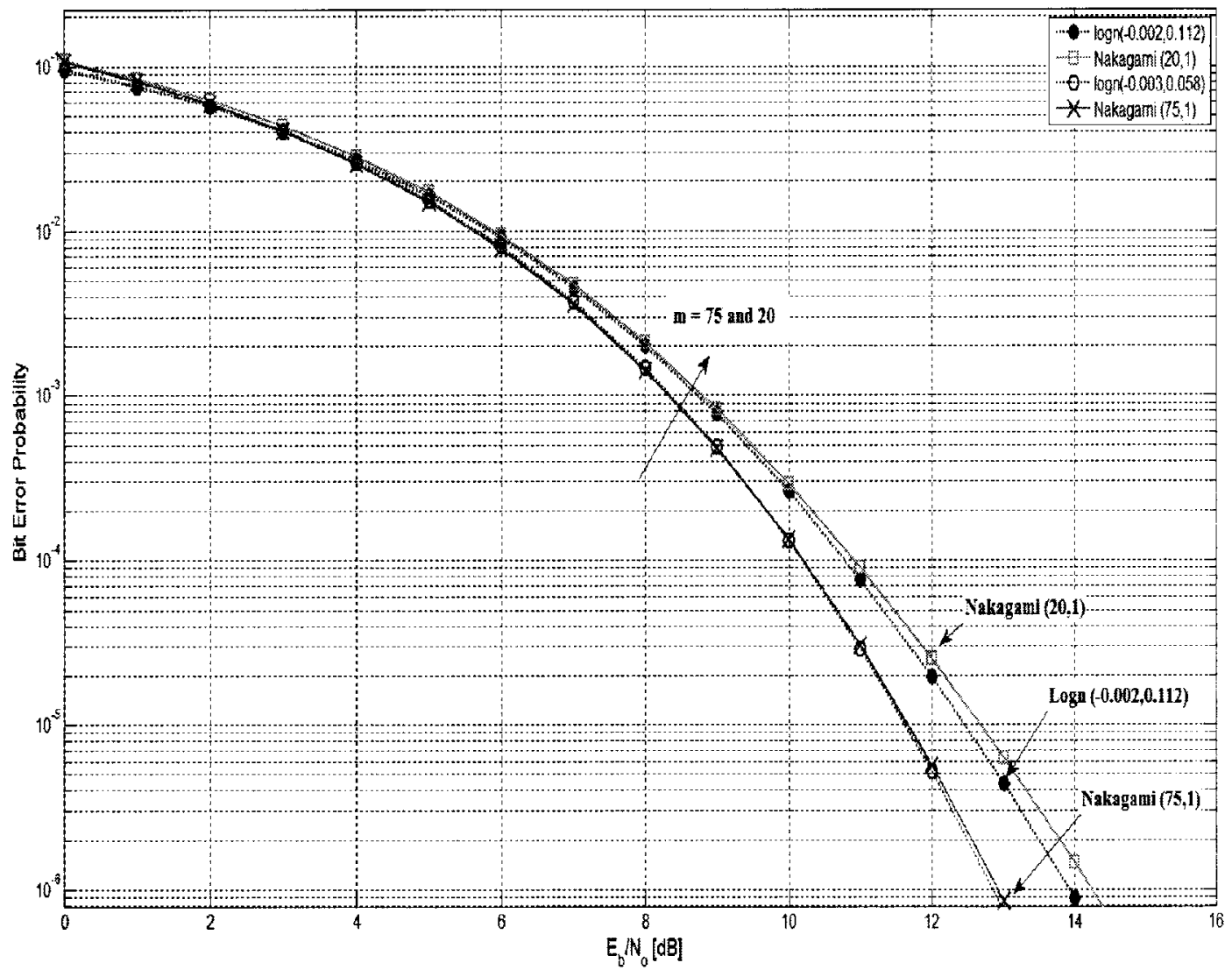

Figure 5.6: TH-PPM BER performance in the presence of a single tone interferer $(\mathrm{SIR}=-10 \mathrm{~dB})$, for $\left(\mathrm{m}=20, \eta_{o}=0.0205, \eta_{1}=1.2289, \eta_{2}=0.9427\right)$, and $(\mathrm{m}=75$, $\left.\eta_{o}=0.0102, \eta_{1}=1.1844, \eta_{2}=0.9526\right)$. 


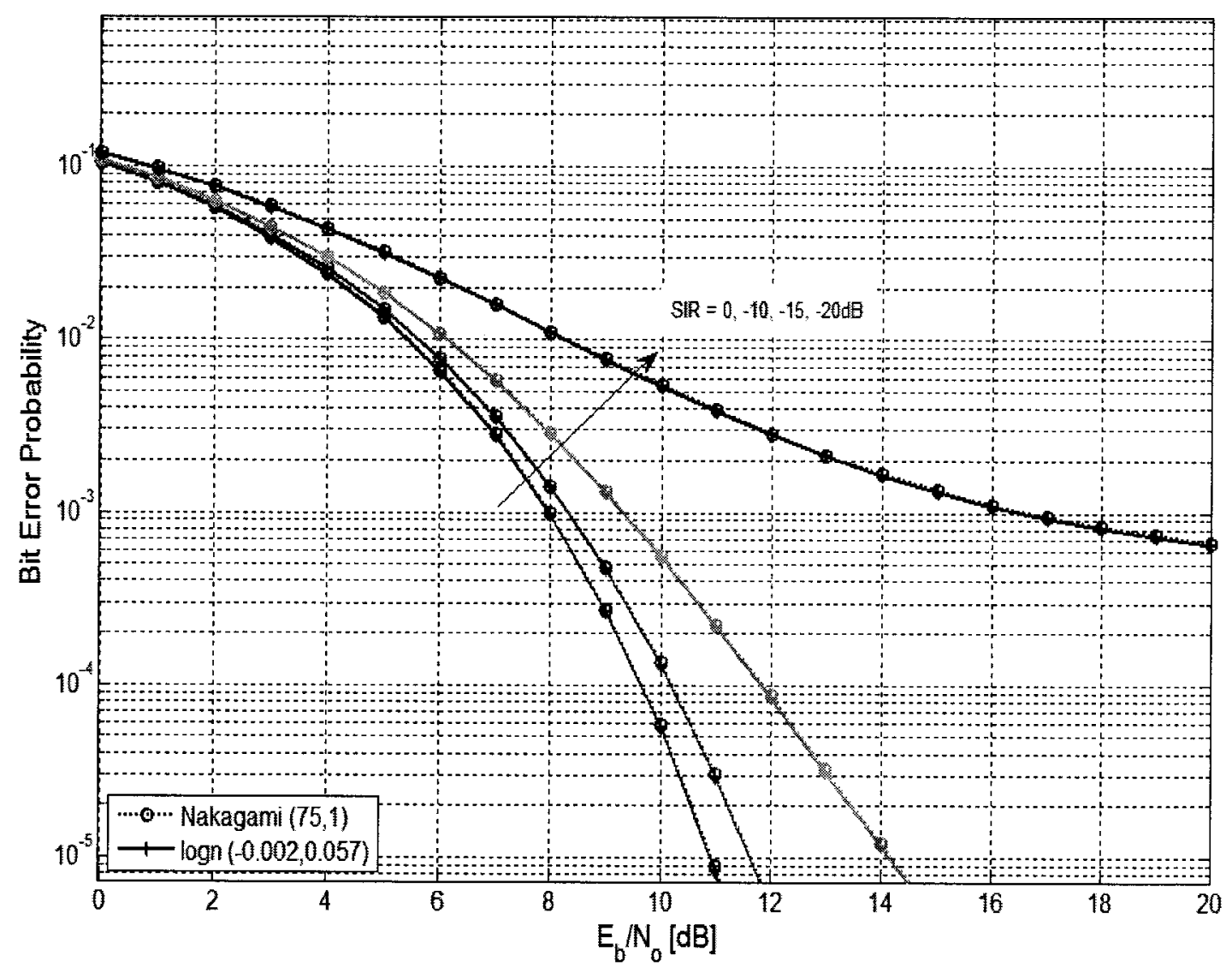

Figure 5.7: TH-PPM performance in a flat fading channel, with a single tone interferer under Log-normal and Nakagami-m distributions for $m=75$. 


\subsubsection{Numerical Results}

With the consideration of the practical values of the $\mathrm{dB}$ spread $[2-5 \mathrm{~dB}]$ for UWB communication systems, the performance of the TH-PPM system in the presence of a single tone interferer in a Log-normal flat fading channel with $\left(\mu_{r}=0, \sigma_{r}=0.921\right)$ is evaluated and depicted in figure 5.8. It is shown that, the SNR degradation is expected to reach up to $5 \mathrm{~dB}$ by increasing the SIR from 0 to $-15 \mathrm{~dB}$ at $\mathrm{BER}=$ $2 \times 10^{-2}$.

Figure 5.9 depicts the BER performance of a TH-PPM system in a frequency selective fading channel for $\mathrm{L}=8$ paths, $\operatorname{Logn}(0.25,0.5296)$, and a decay rate of $\varepsilon^{\prime}=1 / 3$. The BER performance is evaluated in the presence of a single tone interferer using the closed form approximation presented in equation (5.28).

Validation to our results is achieved by making a comparison with BER performance of a UWB system obtained by averaging over the Q-function presented in equation (5.26). It can be seen that, the two results are in a good agreement with each other at different SIRs. The SNR degradation at a $\mathrm{BER}=1 \times 10^{-2}$ is expected to be less than $4 \mathrm{~dB}$ for a $\mathrm{SIR}=-20 \mathrm{~dB}$.

Finally, figure 5.10 depicts the effect of changing the dB-spread value on the performance of the TH-PPM system at different SIRs $(-10$, and $-15 \mathrm{~dB})$.

It is shown that, increasing the $\mathrm{dB}$-spread value from ( $2 \mathrm{~dB}$ to $5 \mathrm{~dB}$ ) leads to a SNR degradation of 4 and $5 \mathrm{~dB}$ for $\mathrm{SIR}=-10$ and $-15 \mathrm{~dB}$ respectively at a BER $=1 \times 10^{-2}$.

\subsection{BER Analysis of a UWB system in the Pres- ence of NBI in Log-normal Flat Fading Chan- nels}

In this section, the performance of a UWB system in a Log-normal flat fading channel in the presence of NBI is evaluated [83]. The UWB propagation channel is characterized by large number of multi-path components, which implies that the UWB channel can not be modeled as a flat fading channel. However, 


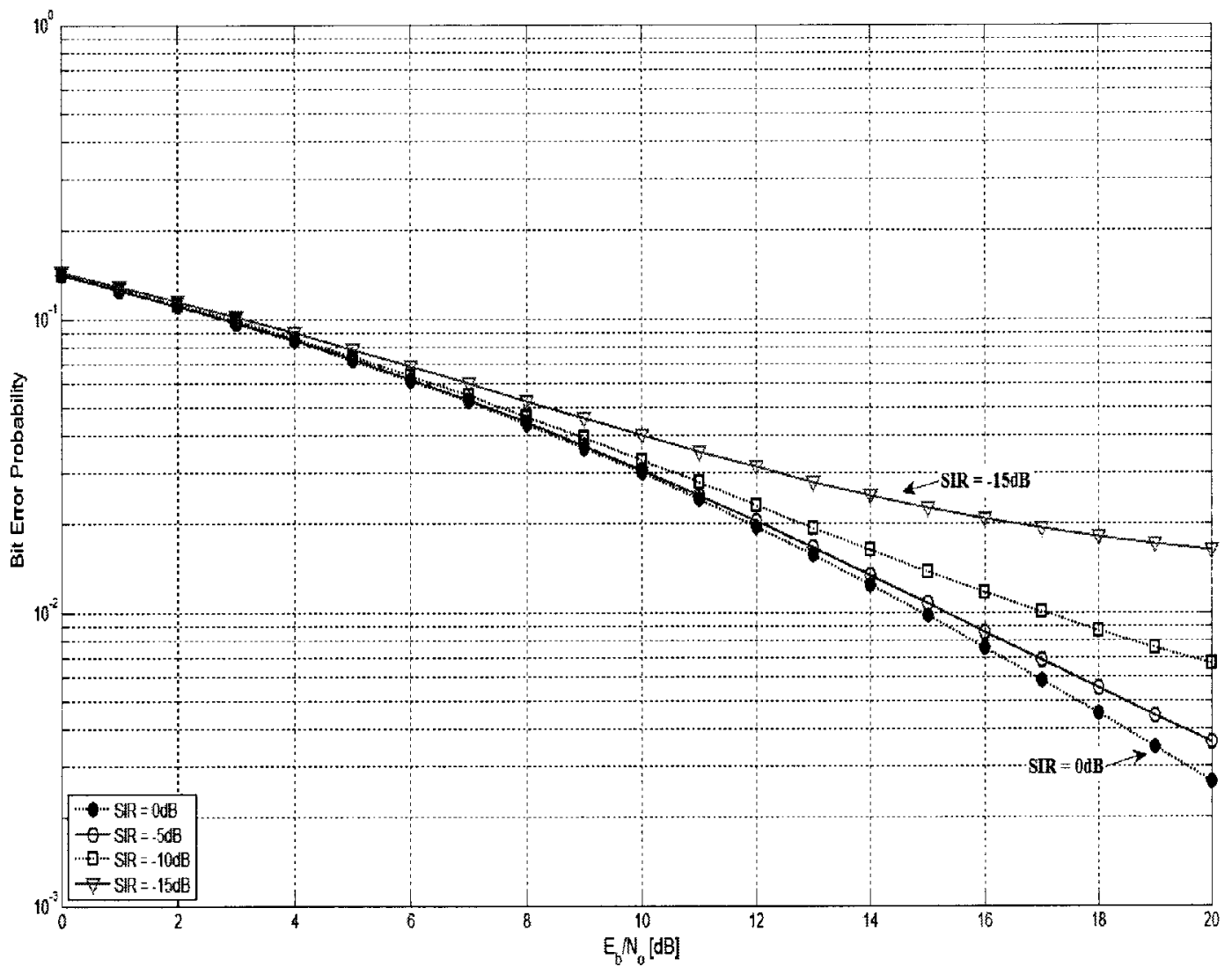

Figure 5.8: TH-PPM performance in the presence of a single tone interferer in a flat fading channel, $\log (0,0.921)$. 


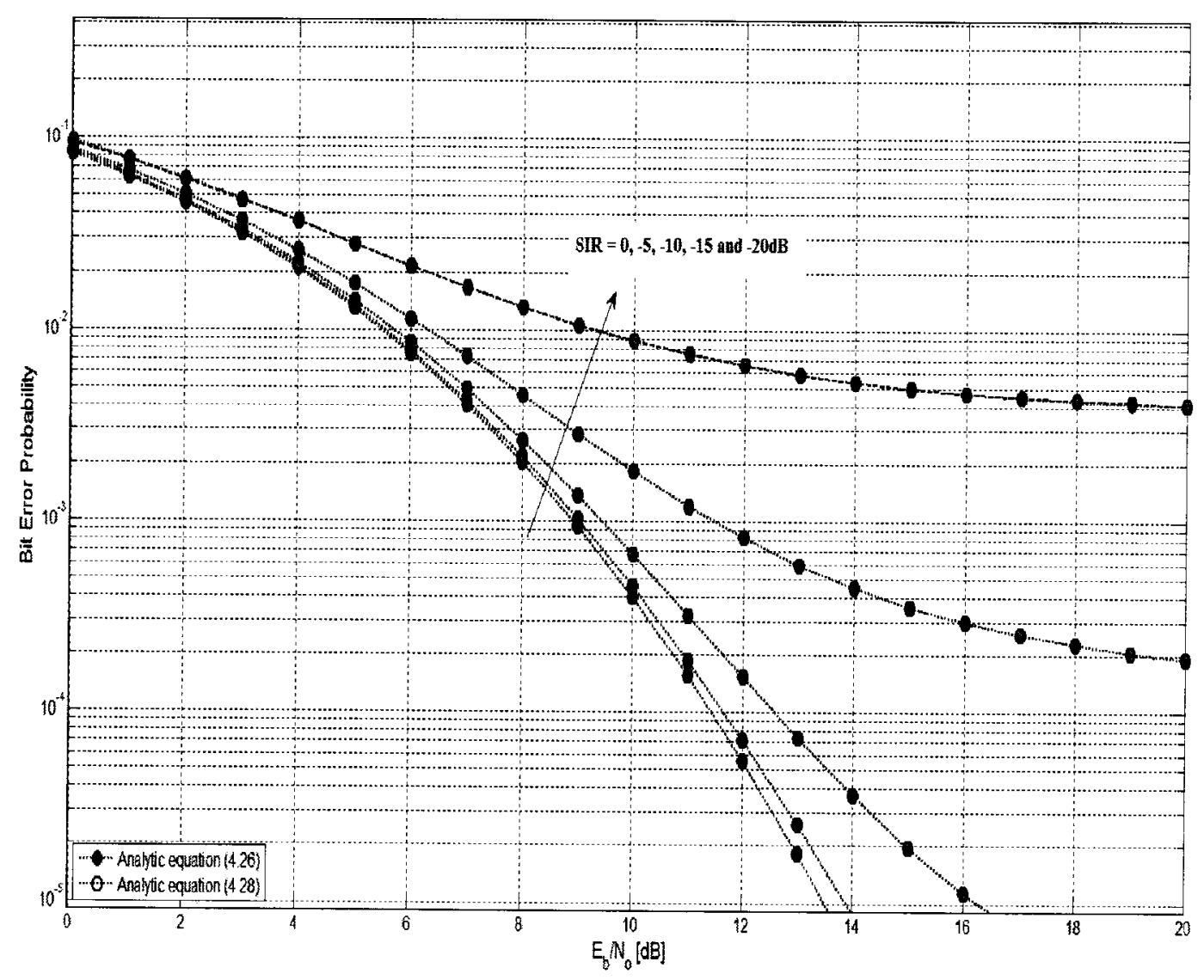

Figure 5.9: TH-PPM performance in the presence of a single tone interferer in a frequency selective fading channel, $\mathrm{L}=8$ paths, $\operatorname{Logn}(0.25,0.5296)$ using relations between equation (5.26) and equation (5.28). 


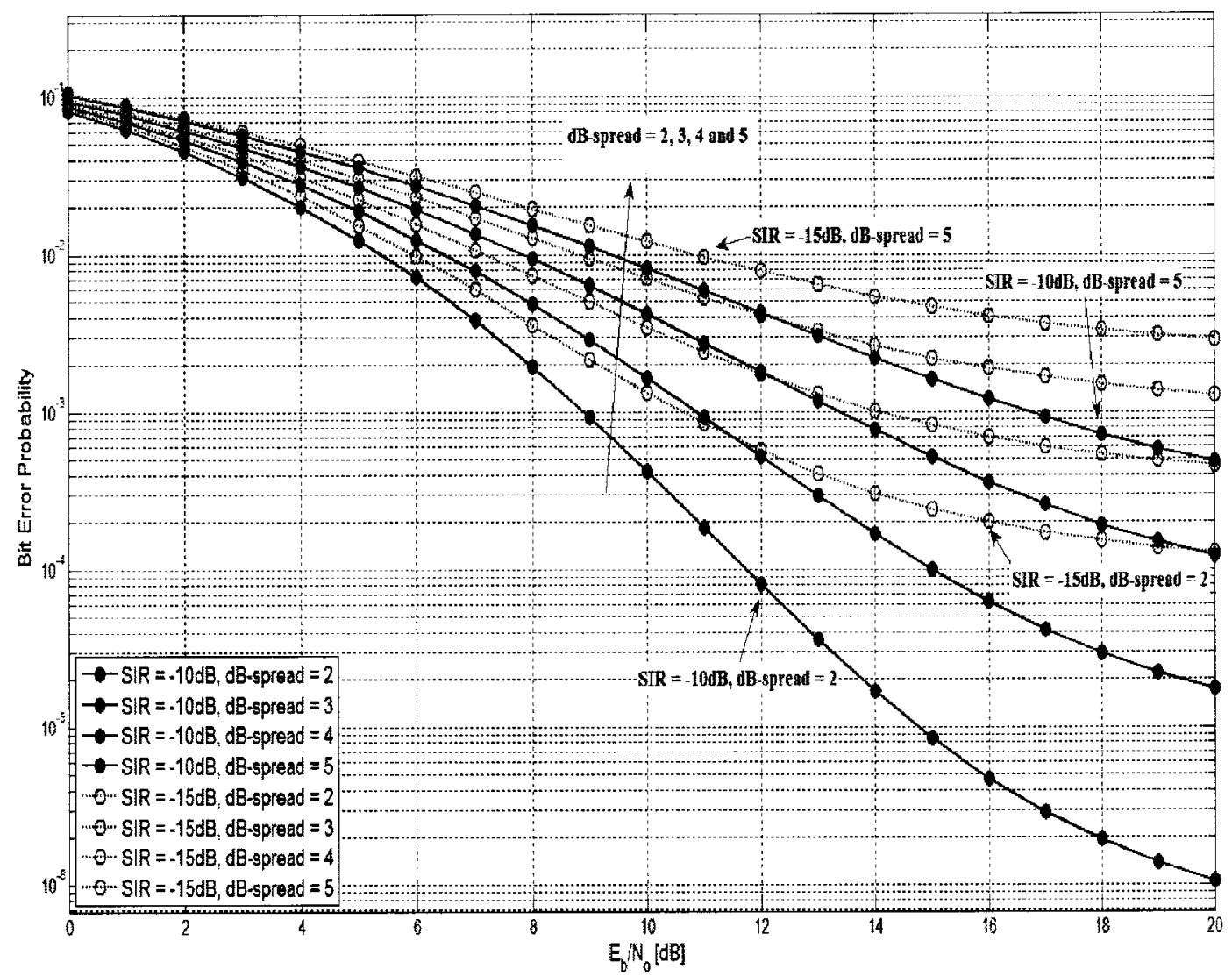

Figure 5.10: The effect of changing the $\mathrm{dB}$ spread values on the performance of the TH-PPM system in presence of a single tone interferer in a frequency selective fading channel, $\mathrm{L}=8$ paths and $\mu_{r}=0.25$. 
some wireless systems that use the UWB technology such as the wireless sensor networks are characterized by size and energy constraints. These constraints are imposed on each node that necessitate the use of simple devices. The use of a finger Rake receiver can be considered as a sub-optimal solution for simple and low cost communication systems. In this case the channel can be modeled as a flat fading.

For a single user binary communication system and a matched filter reception, the transmitted UWB signal can be written either in the form of a TH-PPM or in the form of a DS-BPSK as

$$
\begin{aligned}
& S_{P P M}(t)=\sqrt{E_{b}} \sum_{j=-\infty}^{\infty} p\left(t-j T_{f}-c_{j} T_{c}-\delta d_{\left\lfloor j / N_{s}\right\rfloor}\right) \\
& S_{D S}(t)=\sqrt{E_{b}} \sum_{j=-\infty}^{\infty} d_{j} \cdot c_{j} \cdot p\left(t-j T_{b}-n T_{c}\right)
\end{aligned}
$$

The NBI signal I(t) is assumed to be the sum of $N_{i}$ tones. $\mathrm{I}(\mathrm{t})$ can be written as

$$
I(t)=\sum_{n=1}^{N_{i}} \sqrt{2 I_{n}} \cos \left(2 \pi f_{n} t+\phi_{n}\right)
$$

where $f_{n}$ is the $n^{\text {th }}$ interferer frequency, $\phi_{n}$ is the phase that can be considered as a random variable uniformly distributed over the interval $[0,2 \pi)$, and $I_{n}$ is the transmitted power of the $n^{\text {th }}$ tone signal.

The impulse response of the UWB system in a flat fading channel is given by [20]

$$
h_{s}(t)=a_{s} \delta\left(t-\tau_{s}\right)
$$

where $a_{s}$ is the channel gain coefficient and $\tau_{s}$ is the channel time delay.

The channel impulse response for NBI can be written as

$$
h_{i}(t)=\alpha_{I_{n}} \delta\left(t-\tau_{n}\right)
$$

where $\alpha_{I_{n}}$ is the Rayleigh distributed channel gain and $\tau_{n}$ is the corresponding time delay. 
The overall received signal $r(t)$ can be written as

$$
r(t)=s_{d}(t)+i_{n}(t)+n(t)
$$

where $\mathrm{n}(\mathrm{t})$ is the AWGN with two sided power spectral density $N_{o} / 2$.

For a TH-PPM system, the desired signal $s_{d}(t)=S_{P P M}(t) * h_{s}(t)$ can be written as

$$
s_{d}(t)=\sum_{j=-\infty}^{\infty} a_{s} \sqrt{E_{b}} p\left(t-j T_{f}-c_{j} T_{c}-\delta d_{\left\lfloor j / N_{s}\right\rfloor}\right)
$$

while the interference $i_{n}(t)=I(t) * h_{i}(t)$ can be written as

$$
i_{n}(t)=\sum_{n=1}^{N_{i}} \alpha_{I_{n}} \sqrt{2 I_{n}} \cos \left(2 \pi f_{n}\left(t-\tau_{n}\right)+\phi_{n}\right)
$$

Without loss of generality, it is assumed that the UWB signal channel impulse response $h_{s}(t)$ and the interferer channel gains $\alpha_{I_{n}}$ are normalized so that $E\left[a_{s}^{2}\right]=1$ with $a_{s}^{2}=\int_{-\infty}^{\infty} h_{s}^{2}(t) d t$ and $E\left[\alpha_{I_{n}}^{2}\right]=1$, where $\mathrm{E}[$.$] denotes the expectation operator.$

\subsubsection{Performance Evaluation}

Assuming that the desired signal amplitude undergoes a Log-normal flat fading, whereas the NBI interference signal is affected by Rayleigh fading and the channel is perfectly known at the matched filter receiver.

The correlation mask can be written as

$$
m^{\prime}(t)=a_{s} \cdot m(t)
$$

where $\mathrm{m}(\mathrm{t})$ is presented in equation (5.8).

For a TH-PPM system the transfer function of the matched filter receiver $M(f)$ is presented in equation (3.10), while for a DS-BPSK UWB system; $M(f)$ is given by

$$
|M(f)|=2|P(f)| \sum_{k=0}^{N_{c}-1} c_{k} \exp \left(j 2 \pi f k T_{f}\right)
$$

With the consideration of perfect synchronization with the UWB signal, the decision 
statistic can be written as

$$
\begin{gathered}
Z=\sum_{j=-\infty}^{\infty} \int_{j T_{f}}^{j\left(T_{f}+1\right)} r(t) \cdot m(t) d t \\
Z=S_{p p m}+I_{N}+n
\end{gathered}
$$

where $S_{p p m}, I_{N}$ and $n$ are the desired UWB signal, interference and noise components respectively.

$$
S_{p p m}=\sqrt{E_{b}}(1-\varrho) a_{s}^{2}
$$

The interference term $I_{N}$ can be written as

$$
I_{N}=\sum_{n=1}^{N_{i}} \alpha_{I_{n}} \sqrt{2 I_{n}}\left|M\left(f_{n}\right)\right| \cos \left(\phi_{n}\right)
$$

Each term $\alpha_{I_{n}} \cos \left(\phi_{n}\right)$ is a zero mean Gaussian R.V. with variance $\frac{1}{2}$. Thus, the interference term $I_{N}$ can be considered conditionally Gaussian with variance $\left[\sum_{n=1}^{N_{i}} I_{n}\right.$. $\left.\left|M\left(f_{n}\right)\right|^{2}\right]$

The variance of the noise can be written as

$$
\sigma_{N}^{2}=N_{o}(1-\varrho) a_{s}^{2}
$$

The total disturbance due to the NBI plus noise can be considered conditionally Gaussian with variance $\left[\sum_{n=1}^{N_{i}} I_{n} \cdot\left|M\left(f_{n}\right)\right|^{2}+\sigma_{N}^{2}\right]$.

The conditional BER conditioned on $a_{s}$ can be written as

$$
P_{e \mid a_{s}}=Q\left(\sqrt{a_{s}^{2} \gamma}\right)
$$

where

$$
\gamma=\frac{a_{s}^{2}}{\frac{N_{o}}{E_{b}(1-\varrho)}+\frac{\sum_{n=1}^{N_{i}}\left|M\left(f_{n}\right)\right|^{2}}{S I R \cdot N_{i} \cdot T_{b} \cdot(1-\varrho)^{2} \cdot a_{s}^{2}}}
$$

Since $a_{s}$ is Log-normally distributed, then $a_{s}^{2}$ is Log-normally distributed as well. The 
BER expression is obtained by averaging equation (5.49) over $a_{s}^{2}$ as

$$
P_{e} \simeq \int_{0}^{\infty} Q\left(\sqrt{a_{s}^{2} \gamma}\right) \cdot f\left(a_{s}\right) d a_{s}
$$

where $f\left(a_{s}\right)$ is the PDF of $a_{s}$, which is normally distributed with mean $\mu_{r}$ and variance $\sigma_{r}^{2}, f\left(a_{s}\right)$ can be written as

$$
f\left(a_{s}\right)=\frac{1}{a_{s} \sigma_{r} \sqrt{2 \pi}} \exp \left(\frac{-\left[\ln \left(a_{s}\right)-\mu_{r}\right]^{2}}{2 \sigma_{r}^{2}}\right)
$$

In order to calculate (5.51), we will present the Q-function by the Craig's formula as

$$
\begin{gathered}
Q(x)=\frac{1}{\pi} \int_{0}^{\frac{\pi}{2}} \exp \left(-\frac{x^{2}}{2 \sin ^{2}(\phi)}\right) d \phi \\
P_{e}=\frac{1}{\pi} \int_{0}^{\frac{\pi}{2}}\left[\int_{0}^{\infty} \exp \left(-\frac{a_{s}^{2} \gamma}{2 \sin ^{2}(\phi)}\right) \frac{1}{a_{s} \sigma_{r} \sqrt{2 \pi}} \exp \left(-\frac{\left[\ln \left(a_{s}\right)-\mu_{r}\right]^{2}}{2 \sigma_{r}^{2}}\right) d a_{s}\right] d \phi(5.54)
\end{gathered}
$$

By letting $\mathrm{x}=\frac{\ln \left(a_{s}\right)-\mu_{r}}{\sqrt{2} \sigma_{r}}, P_{e}$ will become

$$
P_{e}=\frac{1}{\pi} \int_{0}^{\frac{\pi}{2}}\left[\frac{1}{\sqrt{\pi}} \int_{-\infty}^{\infty} e^{-x^{2}} \cdot \exp \left(-\frac{\gamma}{2 \sin ^{2}(\phi)} \cdot \exp \left[2 \sqrt{2} x \sigma_{r}+2 \mu_{r}\right]\right) d x\right] d \phi
$$

The inner integral in equation (5.55) can be approximated by a Gauss-Hermite series expansion to become

$$
=\frac{1}{\sqrt{\pi}} \sum_{i=1}^{N} w_{i} \exp \left(-\frac{\gamma}{2 \sin ^{2}(\phi)} \exp \left[2 \sqrt{2} b_{i} \sigma_{r}+2 \mu_{r}\right]\right) d \phi
$$

where $w_{i}$ and $b_{i}$ are the weights and the associated roots of the Hermite polynomial respectively where $w_{i}$ and $b_{i}$ are found in [84]. $\mathrm{N}$ are the number of samples points to use for this approximation. 
By substituting equation (5.56) into equation (5.55) we will get

$$
P_{e}=\frac{1}{\sqrt{\pi}} \sum_{i=1}^{N} w_{i}\left[\frac{1}{\pi} \int_{0}^{\frac{\pi}{2}} \exp \left(-\frac{\gamma}{2 \sin ^{2}(\phi)} \cdot \exp \left(2 \sqrt{2} b_{i} \sigma_{r}+2 \mu_{r}\right)\right)\right] d \phi
$$

which can be rewritten as

$$
\left.P_{e}=\frac{1}{\sqrt{\pi}} \sum_{i=1}^{N} w_{i}\left[Q\left(\sqrt{\gamma \cdot \exp \left(2 \sqrt{2} b_{i} \sigma_{r}+2 \mu_{r}\right.}\right)\right)\right]
$$

\subsubsection{Numerical Results}

Now, the analytical expression developed in the previous section (equation 5.58) is used to evaluate the performance of both the TH-PPM and DS-BPSK UWB systems under the impact of a single tone interferer in a Log-normal flat fading channel. A second derivative Gaussian received pulse with the same values suggested in [71] is considered, where for a TH-PPM scheme: $T_{f}=100 \mathrm{~ns}, \tau_{p}=0.5 \mathrm{~ns}, \delta=0.3 \mathrm{~ns}$. and $N_{s}=2$ pulses/bit. The correlation parameter $\varrho=-0.587$ and $T_{c}=1.5$ ns. A user with a TH sequence of all zeros, i.e. $c_{o}=c_{1}=0$. The interferer frequency $f_{i}=2.412$ GHz.

Figure 5.11 depicts the performance of the TH-PPM system in the presence of a single tone interferer at different SIRs. The evaluation of the closed form approximation presented in equation (5.58) is compared with the analytic results obtained by averaging over the Q-function presented in equation (5.51). The derived analytic results are also validated with the aid of simulation. It can be seen that a good agreement among these results is accomplished.

Figure 5.12 depicts the effect of changing the dB-spread value on the performance of the TH-PPM system at $\mathrm{SIR}=-10 \mathrm{~dB}$. The SNR degradation is expected to reach up to $10 \mathrm{~dB}$ at $\mathrm{BER}=2 \times 10^{-2}$ by increasing the value of the $\mathrm{dB}$-spread from $2 \mathrm{~dB}$ to $5 \mathrm{~dB}$.

For a DS-BPSK-UWB system, by considering the same values taken in the TH-PPM case except: the frame duration $T_{f}=25 \mathrm{~ns}, N_{c}=8 \mathrm{chip} / \mathrm{bit}$, so we will obtain the same bit duration $T_{b}=200 \mathrm{~ns}$ in both cases. The chip code $c_{k}=(-1)^{k}, \mathrm{k}=1, \ldots, N_{c}$. 


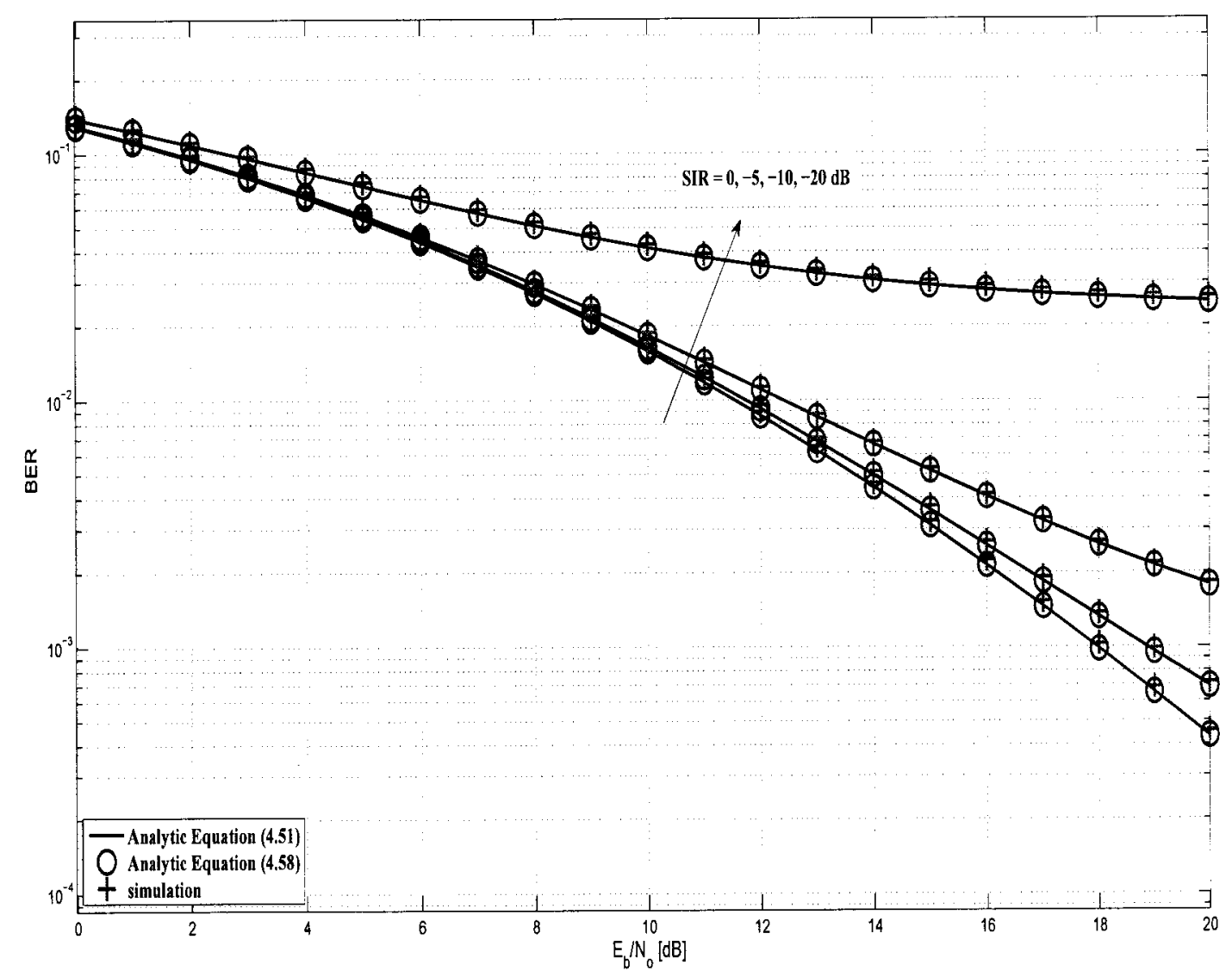

Figure 5.11: BER performance of the TH-PPM system in a Log-normal flat fading channel in the presence of a single tone interferer compared with the analytic result of equation (5.51), dB-spread $=3 \mathrm{~dB}$. Analytic results are also compared with simulation. 


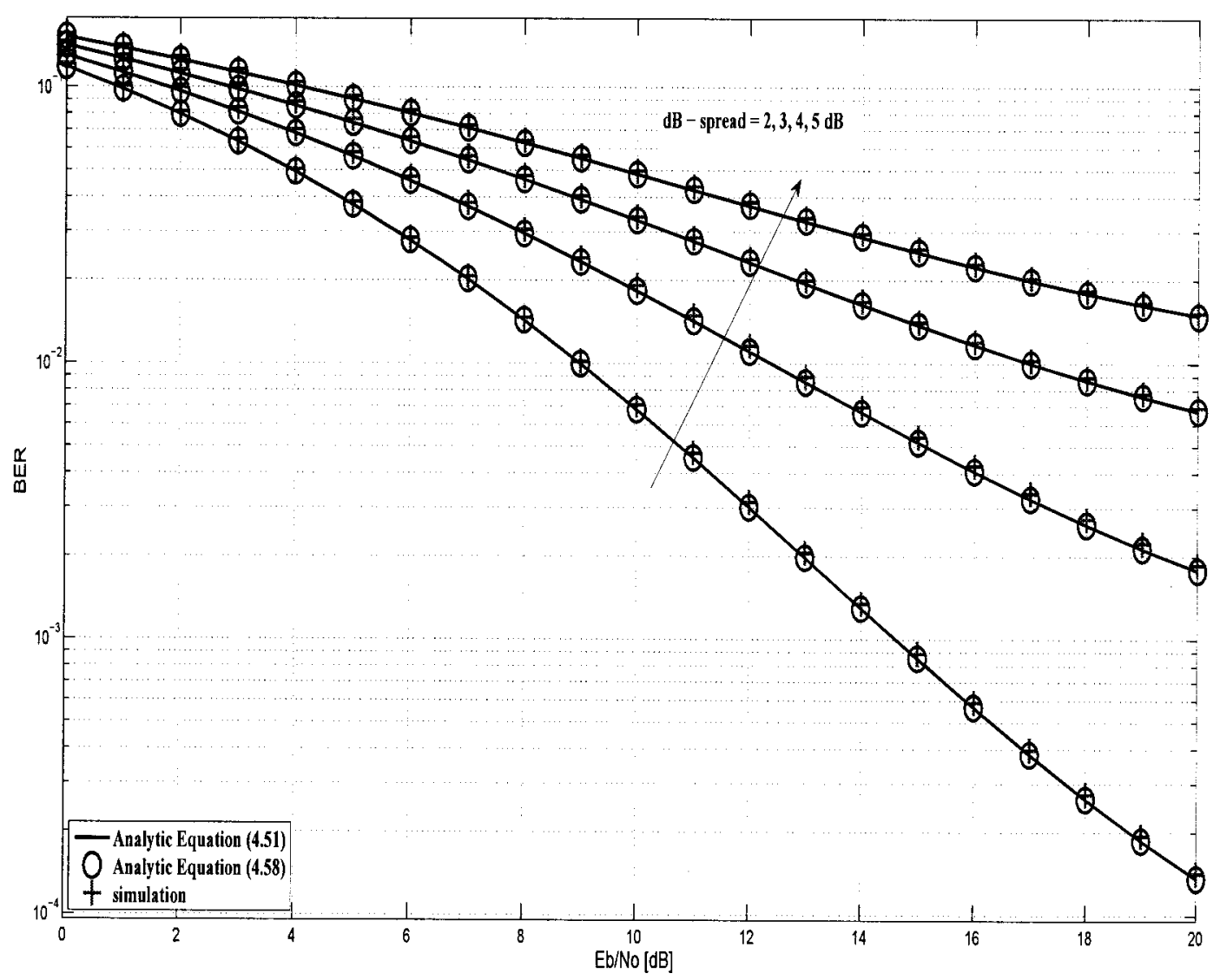

Figure 5.12: BER performance of the TH-PPM system in the presence of a single tone interferer at different dB-spread values [2-5 dB]. Analytic results are also compared with simulation. 


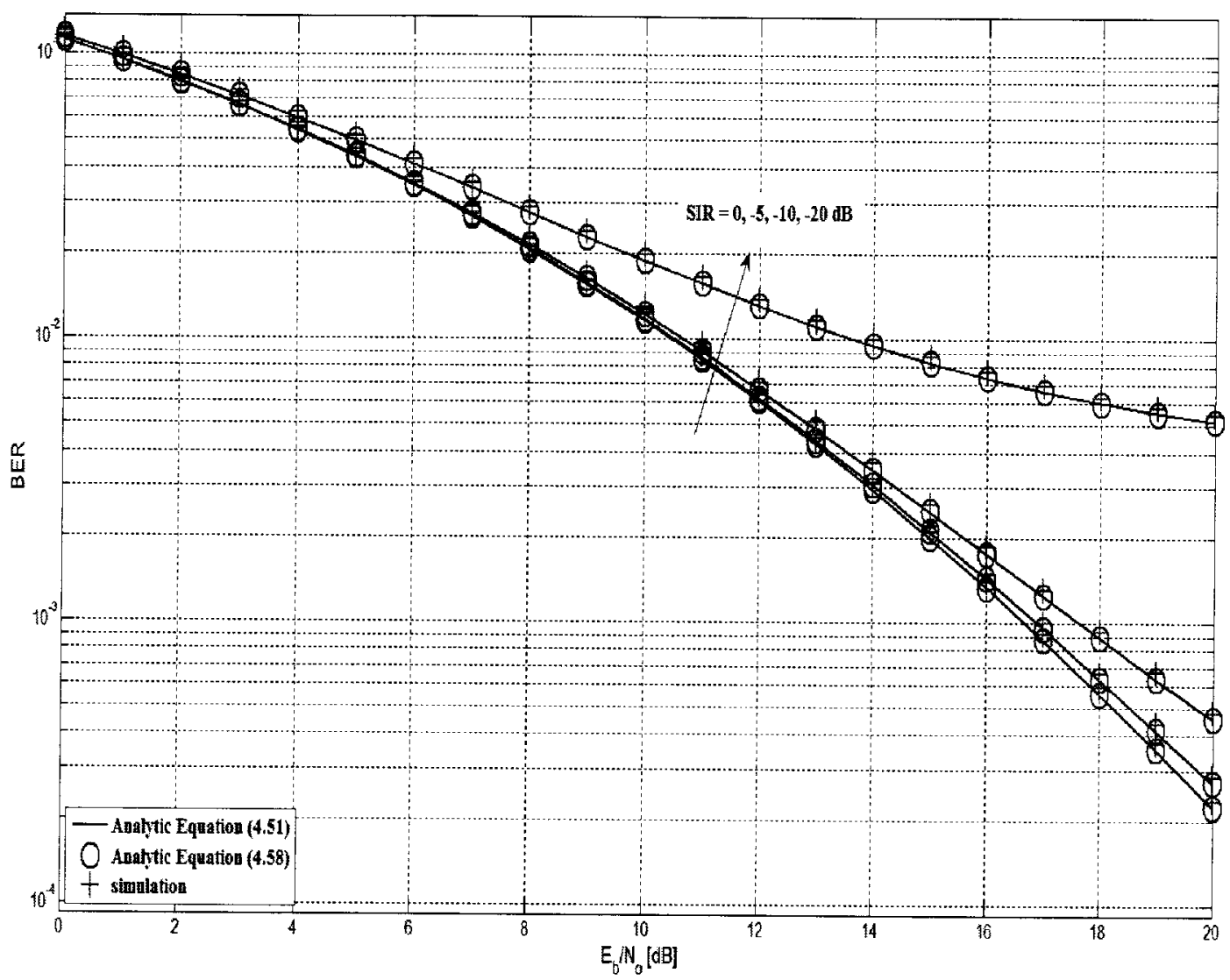

Figure 5.13: BER performance of the DS-BPSK system in a Log-normal flat fading channel in the presence of a single tone interferer, $\mathrm{dB}$-spread $=3 \mathrm{~dB}$. Analytic results are compared with simulation and with the analytic results obtained from equation (5.51). 
Figure 5.13 depicts the performance of such system in the presence of a single tone interferer. By comparing these results with the results presented in figure 5.11, it can be seen that the DS system outperforms the TH one in the presence of interference in a Log-normal flat fading channel.

The impact of the presence of two tone interferers on the performance of a TH-PPM system is depicted in figure 5.14 and compared with the single tone case.

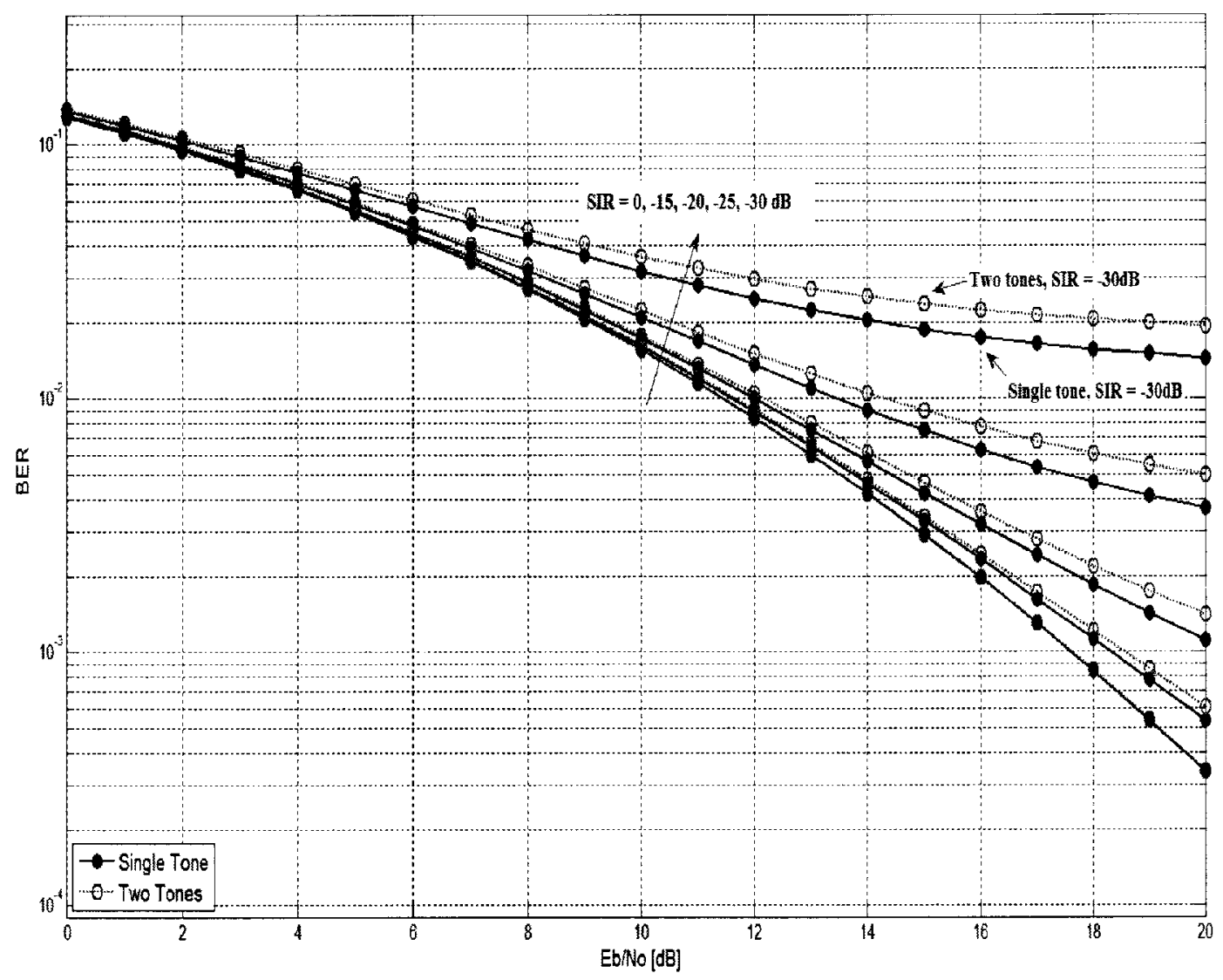

Figure 5.14: Single tone versus two tone interferers impact on the TH-PPM system. $\mathrm{dB}$-spread $=3 \mathrm{~dB}$. 


\subsection{BER Analysis of a UWB system in the Pres- ence of NBI in the IEEE802.15.3a UWB Chan- nel Models}

In this section, the BER performance of the Rake reception of the UWB signal in the presence of NBI in the IEEE802.15.3a UWB channel models is evaluated [85]. The NBI signal is modeled as the standard IEEE802.11a WLAN, where this multi-carrier based interference signal is approximated as sum of $N_{i}$ tone interferers.

A single user in a binary communication system is considered. The transmitted UWB signal is modeled as a DS-BPSK signal, which can be written as [26]

$$
S_{D S}(t)=\sum_{j=-\infty}^{\infty} \sum_{m=0}^{N_{c}-1} b_{j} \cdot C_{m, j} \cdot p\left(t-j T_{b}-m T_{c}\right)
$$

The energy of the UWB pulse can be represented as $E_{p}=\int_{-\infty}^{\infty} p^{2}(t) d t$, with a normalized autocorrelation function $R(\kappa)=\frac{1}{E_{p}} \int_{-\infty}^{\infty} p(t) p(t-\kappa) d t$

The NBI signal I(t) can be approximated by $N_{i}$ tone interferers ${ }^{4}$ as suggested in $[27]$

$$
I(t)=\sqrt{\frac{2 I}{N_{i}}} \sum_{n=0}^{N_{i}-1} \cos \left(2 \pi f_{n} t+\phi_{n}\right)
$$

where $\phi_{n}$ are independent and identically distributed (i.i.d.) random phases due to modulation and i.i.d. symbols and $I$ is the total transmitted power of the interference.

According to [28], it had been recognized in many UWB multi-path channel investigations that multi-path components tend to arrive in clusters of rays. The most popular way to reflect this mathematically is the $\mathrm{S}-\mathrm{V}$ multi-path channel model and its modifications [86]. The discrete time impulse response of the multi-path

\footnotetext{
${ }^{4}$ The $N_{i}$ tone interferers are equivalent to the number of the OFDM data sub-carriers.
} 
UWB channel is given by [28]

$$
h_{s}(t)=\sum_{\ell=0}^{L} \sum_{k=0}^{K} a_{k, \ell} \cdot \delta\left(t-T_{\ell}-\tau_{k, \ell}\right)
$$

where $\ell$ is the cluster index, and $\mathrm{k}$ is the ray index within a cluster, the total number of clusters and rays are donated by $\mathrm{L}$ and $\mathrm{K}$ respectively. $a_{k, \ell}$ is the multi-path fading coefficient of the of the $k^{t h}$ ray within the $\ell^{\text {th }}$ cluster and $\delta($.$) is the Kronecker Delta.$ The arrival time of the $\ell^{\text {th }}$ cluster is denoted by $T_{\ell}$, and that of the $k^{\text {th }}$ ray within the $\ell^{\text {th }}$ cluster is denoted by $\tau_{k, \ell}$.

It was reported in [87], that the arrival time of clusters and rays are modeled by a Poisson distribution, thus the PDF of $T_{\ell}$ for the CM1 channel model (line-of-sight "LOS"), where a LOS component arrives at time 0 , can be written as

$$
\wp_{T_{\ell}}=\Lambda \cdot e^{(-\Lambda t)} \cdot \frac{(\Lambda t)^{\ell-1}}{(\ell-1) !}
$$

Whereas, for the CM2, CM3 and CM4 (Non-line-of-sight "NLOS") channel models, the PDF can be written as

$$
\wp_{T_{\ell}}=\Lambda \cdot e^{(-\Lambda t)} \cdot \frac{(\Lambda t)^{\ell}}{\ell !}
$$

where $\Lambda$ is the cluster average arrival rate, the PDF of $\tau_{k, \ell}$ can be written as

$$
\wp_{\tau_{k, \ell}}=\lambda \cdot e^{(-\lambda t)} \cdot \frac{(\lambda t)^{k-1}}{(k-1) !}
$$

where for $\mathrm{k}=0, \tau_{0, \ell}=0 . \lambda$ is the ray arrival rate.

The channel fading coefficients $a_{k, \ell}$ can be defined as [2]

$$
a_{k, \ell}=p_{k, \ell} \xi_{\ell} \beta_{k, \ell}
$$

$p_{k, \ell}$ is a discrete R.V., $p_{k, \ell} \in\{-1,+1\}$ with equal probability to account for pulse inversion due to reflection, $\xi_{\ell}$ and $\beta_{k, \ell}$, stand for the fading associated with the $\ell^{t h}$ cluster and the $k^{\text {th }}$ ray within the $\ell^{t h}$ cluster respectively. The product $\xi_{\ell} \beta_{k, \ell}$ can be considered as a random variable that is Log-normally distributed [5] $20 \log _{10}\left(\xi_{\ell} \beta_{k, \ell}\right) \propto N\left(\mu_{k, \ell}, \sigma_{1}^{2}+\sigma_{2}^{2}\right)$, where $\mu_{k, \ell}$ is presented in equation (2.27). 
For the narrowband interferer, the channel impulse response can be written as

$$
h_{i}(t)=\sum_{n=0}^{N_{i}-1} \alpha_{I_{n}} \delta\left(t-\tau_{n}\right)
$$

where $\alpha_{I_{n}}$ are the Rayleigh distributed channel gains and $\tau_{n}$ are the corresponding time delays.

The overall received signal $r(t)$ can be written as

$$
r(t)=S_{r}(t)+I_{i}(t)+n(t)
$$

where the desired received UWB signal $S_{r}(t)=\sum_{\ell=0}^{L} \sum_{k=0}^{K} a_{k, \ell} \cdot S_{D S}\left(t-T_{\ell}-\tau_{k, \ell}\right)$ and $\mathrm{n}(\mathrm{t})$ is the noise signal with zero mean and variance $\sigma_{n}^{2}$.

The received NBI signal $I_{i}(t)$ can be written as

$$
I_{i}(t)=\sqrt{\frac{2 I}{N_{i}}} \sum_{n=0}^{N_{i}-1} \alpha_{I_{n}} \cos \left[2 \pi f_{n}\left(t-\tau_{n}\right)+\phi_{n}\right]
$$

\subsubsection{Performance Evaluation}

The performance of the DS-BPSK system in the presence of NBI in a frequency selective fading channel is evaluated. The UWB signal undergoes Log-normal fading, whereas the NBI signal is affected by Rayleigh fading. Assuming that the channel is perfectly known and in order to clarify the role of NBI on the performance the UWB system we assume that there is no inter-symbol interference, inter-path interference, or inter-chip interference, where their impact have been presented in [13].

In the MRC Rake receiver, the received signal of the $j^{\text {th }}$ data symbol is correlated with the correlation mask $m^{\prime}(t)$, which can be written as

$$
m^{\prime}(t)=\sum_{\ell=0}^{L} \sum_{k=0}^{K} a_{k, \ell} \cdot m(t)
$$

For a DS-BPSK system $M(f)$ is the F.T. of $\mathrm{m}(\mathrm{t})$ as represented in equation (5.43), where $C_{m}=(-1)^{m}, m=0, \ldots, N_{c}-1$, and $P(f)$ is the Fourier Transform of the 
UWB pulse $\mathrm{p}(\mathrm{t})$, which can be modeled as the six derivative Gaussian pulse as presented in equation (3.15).

With the consideration of perfect synchronization with the UWB signal, the decision statistic can be written as

$$
\begin{gathered}
Z=\sum_{j=-\infty}^{\infty} \int_{j T_{f}}^{j\left(T_{f}+1\right)} r(t) \cdot m^{\prime}(t) d t \\
Z=S_{p p m}+i_{n}+n
\end{gathered}
$$

where $S_{p p m}, i_{n}$ and $n$ are the desired signal, interference and noise components respectively.

$$
S_{p p m}=N_{c} E_{p} \sum_{\ell=0}^{L} \sum_{k=0}^{K}\left|a_{k, \ell}\right|^{2}
$$

- Statistics of the UWB Signal

It was suggested in [88], that the mean of the desired UWB signal component in the CM1 LOS channels can be written as

$$
E\left\{S_{p p m}\right\}=N_{c} E_{p} \beta_{o, o}(1+\Gamma \Lambda)(1+\gamma \lambda) \cdot\left[1-\left(\frac{\Gamma \Lambda}{1+\Gamma \Lambda}\right)^{L+1}\right] \cdot\left[1-\left(\frac{\gamma \lambda}{1+\gamma \lambda}\right)^{K+1}\right]
$$

whereas, for NLOS channels (CM2, CM3 and CM4) $E\left\{S_{p p m}\right\}$ can be calculated as

$$
E\left\{S_{p p m}\right\}=N_{c} E_{p} \beta_{o, o}(\Gamma \Lambda)(1+\gamma \lambda) \cdot\left[1-\left(\frac{\Gamma \Lambda}{1+\Gamma \Lambda}\right)^{L+1}\right] \cdot\left[1-\left(\frac{\gamma \lambda}{1+\gamma \lambda}\right)^{K+1}\right]
$$

The second order moment of $S_{p p m}$ is given by [88]

$$
E\left\{S_{p p m}^{2}\right\}=N_{c}^{2} E_{p}^{2} \sum_{\ell=0}^{L} \sum_{k=0}^{K} \sum_{\ell^{\prime}=0}^{L} \sum_{k^{\prime}=0}^{K} E\left(a_{k, \ell}^{2} \cdot a_{k^{\prime}, \ell^{\prime}}^{2}\right)
$$

where $E\left(a_{k, \ell}^{2} \cdot a_{k^{\prime}, \ell^{\prime}}^{2}\right)$ can be evaluated for two cases: the first one is where $\left(\ell=\ell^{\prime}\right)$,

$$
E\left(a_{k, \ell}^{2} \cdot a_{k^{\prime}, \ell^{\prime}}^{2}\right)=\beta_{o, o}^{2} \exp \left[\frac{(\ln 10)^{2} \sigma_{1}^{2}}{100}\right] E\left(\ell, \ell, k, k^{\prime}\right), \quad k \geq k^{\prime}
$$




$$
E\left(x_{1}, x_{2}, x_{3}, x_{4}\right)=\left(\frac{\Gamma \Lambda}{1+\Gamma \Lambda}\right)^{x_{1}}\left(\frac{1+\Gamma \Lambda}{2+\Gamma \Lambda}\right)^{x_{2}}\left(\frac{\gamma \lambda}{1+\gamma \lambda}\right)^{x_{3}}\left(\frac{1+\gamma \lambda}{2+\gamma \lambda}\right)^{x_{4}}
$$

For $\left(k^{\prime}>k\right)$, we just switch $k^{\prime}$ with $\mathrm{k}$. The second case, where $\left(\ell \neq \ell^{\prime}\right)$ one has

$$
E\left(a_{k, \ell}^{2} \cdot a_{k^{\prime}, \ell^{\prime}}^{2}\right)=\beta_{o, o}^{2} E\left(\ell, \ell, k+k^{\prime}, 0\right), \quad \ell \geq \ell^{\prime}
$$

For the scenario where $\left(\ell^{\prime}>\ell\right)$, we just switch $\ell^{\prime}$ with $\ell$.

As suggested in [88] and [89], the UWB signal component, $S_{p p m}$, can be well approximated by a Log-normal distributed R.V. that can be well characterized by $\left[10 \log _{10}\left(S_{p p m}\right) \propto N\left(\mu_{s}, \sigma_{s}^{2}\right)\right]$, where according to the Wilkinson approximation method [76]

$$
\begin{aligned}
& \mu_{s}=\frac{10}{\ln (10)} \ln \left(\frac{E^{2}\left(S_{p p m}\right)}{\sqrt{E\left(S_{p p m}^{2}\right)}}\right), \\
& \sigma_{s}^{2}=\frac{100}{(\ln 10)^{2}} \ln \left(\frac{E^{2}\left(S_{p p m}\right)}{E\left(S_{p p m}^{2}\right)}\right) .
\end{aligned}
$$

- Statistics of the Interference

The interferer term $i_{n}$ can be written as

$$
i_{n}=\sqrt{\frac{2 I}{N}} \sum_{n=0}^{N-1} \alpha_{I_{n}} \cdot\left|M\left(f_{n}\right)\right| \cdot\left|H_{s}\left(f_{n}, \mathbf{a}, \mathbf{T}, \mathbf{t}\right)\right| \cos \left(\phi_{n}\right)
$$

Let the R.V. $\zeta=\sum_{\ell=0}^{L} \sum_{k=0}^{K} a_{k, \ell} \cdot e^{-j \varphi_{k, \ell}}$, where we can define $\varphi_{k, \ell}=2 \pi f\left(T_{\ell}+\tau_{k, \ell}\right)$.

As depicted in table 2.3, the average cluster plus ray arrival time through the IEEE UWB channel models ranges between [15 - 43] ns, while the OFDM based IEEE802.11a NBI; operates in the U-NII $5 \mathrm{GHz}$ radio bands. The product $f\left(T_{\ell}+\tau_{k, \ell}\right)$, will be much greater than unity. This suggests the assumption of considering $\varphi_{k, \ell}$ as i.i.d. R.V.s uniformly distributed over $[0,2 \pi)$.

We can consider the phase term $\left[\arg \left[H\left(f_{n}\right)\right]+2 \pi f_{n}\left(t-\tau_{n}\right)\right]$ is included within the random phase $\phi_{n}$.

The interference term $i_{n}$ is conditionally Gaussian $\left[\mathrm{N}\left(0, \sigma_{i}^{2}\right)\right]$, conditioned on the R.V. 
$\left[\left|M\left(f_{n}\right)\right| \cdot\left|H_{s}\left(f_{n}, \mathbf{a}, \mathbf{t}\right)\right|\right]$ with variance $\sigma_{i}^{2}=\frac{I}{N} \sum_{n=0}^{N-1}\left|M\left(f_{n}\right)\right|^{2} \cdot\left|H_{s}\left(f_{n}, \mathbf{a}, \mathbf{t}\right)\right|^{2}$.

The total disturbance due to the NBI plus noise is also conditionally Gaussian with variance, $\left[\sigma_{i}^{2}+\sigma_{n}^{2}\right]$.

By letting $a_{s}=\sum_{\ell=0}^{L} \sum_{k=0}^{K} a_{k, \ell}$, the conditional BER conditioned on $(\mathbf{a}, \mathbf{t})$ can be written as $P_{e \mid \mathbf{a}, \mathbf{t}}=Q(\sqrt{\gamma(\mathbf{a}, \mathbf{t})})$, where $\gamma(\mathbf{a}, \mathbf{t})=\frac{N_{c} E_{g} a_{s}^{2}}{\sigma_{n}^{2}+\sigma_{i}^{2}}$.

By considering a R.V. $\zeta=\left|H_{s}\left(f_{n}, \mathbf{a}, \mathbf{t}\right)\right|$ and under the assumption of fixed but arbitrary path gains a averaging over the R.V. $\zeta$ can be approximated with the aid of "Stirling formula" as suggested in [75].

The statistics of $\varphi_{k, \ell}$ for a fixed a can be evaluated as [71]

$$
\begin{aligned}
& \mu(\mathbf{a})=a_{s}^{2} \\
& \sigma^{2}(\mathbf{a})=(1-\varpi) a_{s}^{4} \\
& v(\mathbf{a})=(2-6 \varpi+4 y) a_{s}^{6}
\end{aligned}
$$

For a normalized power dispersion profile $\aleph_{k, \ell}, \varpi=\sum_{\ell=0}^{L} \sum_{k=0}^{K} \aleph_{k, \ell}^{2}$ and $y=\sum_{\ell=0}^{L} \sum_{k=0}^{K} \aleph_{k, \ell}^{3}$, where $\aleph_{k, \ell}=E\left(a_{k, \ell}^{2}\right)$.

The conditional BER over the path gains a can be written as

$$
P_{e} \mid \mathbf{a} \simeq \sum_{n=1}^{N} b_{n} \cdot Q\left(\sqrt{\gamma\left(q_{n} a_{s}^{2}\right)}\right)
$$

$\mathrm{N}$ is the number of terms in the expansion. The weights $\mathbf{b}$ and $\mathbf{q}$ are:

$$
\begin{aligned}
\mathbf{b} & =\left[1 / 6+\xi_{1}, 2 / 3,1 / 6-2 \xi_{1}, \xi_{1}\right], \\
\mathbf{q} & =\left[0,1,1+\sqrt{3} \xi_{2}, 1+2 \sqrt{3} \xi_{2}\right], \\
\xi_{1} & =(1-\varpi), \\
\xi_{2} & =[1-3 \varpi+2 y] /\left[18 \sqrt{3}(1-\varpi)^{\frac{3}{2}}\right]
\end{aligned}
$$


By averaging equation (5.82) over a, the BER can be written as

$$
P_{e} \simeq \int_{0}^{\infty} P_{e} \mid \chi \cdot f(\chi) d \chi
$$

where $f(\chi)$ is the PDF given by [88]

$$
f(\chi)=\frac{10}{\chi \ln (10) \sqrt{2 \pi \sigma_{s}^{2}}} \exp \left[\frac{-\left(10 \log _{10}(\chi)-\mu_{s}\right)^{2}}{2 \sigma_{s}^{2}}\right]
$$

\subsubsection{Numerical Results}

A six derivative Gaussian received pulse is used with values: $\tau_{p}=0.192$ ns and a bit duration $T_{b}=200 \mathrm{~ns}$. Assuming $\beta_{o, o}=1, \sigma_{1}=\sigma_{2}=3.3941 \mathrm{~dB}, \mathrm{~L}=2$ clusters, $\mathrm{K}=$ 20 rays/cluster.

The IEEE802.11a NBI signal will operate at the upper U-NII band with center frequency $=5.745 \mathrm{GHz}$, and the frequency spacing between the carriers $\Delta f=0.3125$ $\mathrm{MHz}$.

Figures 5.15 and 5.16 depict the performance of a MRC Rake receiver for the DS-UWB system in presence of the standard IEEE802.11a NBI signal in the CM1, and the CM4 IEEE channel models respectively at different SIRs.

SNR degradation of $4 \mathrm{~dB}$, and $12 \mathrm{~dB}$ is expected in the CM1 and the CM4 channel models respectively at $\mathrm{BER}=3 \times 10^{-2}$ by changing the SIR from $0 \mathrm{~dB}$ to $30 \mathrm{~dB}$.

A validation to the derived analytical results is achieved in two scenarios: the first, a comparison with the results obtained by simulating the performance of the DS-UWB system in presence of the IEEE802.11a NBI signal in the CM1 and the $\mathrm{CM} 4$ for $\mathrm{SIR}=0,-15,-20$ and $-30 \mathrm{~dB}$ is depicted in figure 5.17.

The second, a comparison with the simulation experiment suggested in [12] in the CM1 channel model for 10-fingers MRC Rake receiver is depicted in figure 5.18. In this simulation experiment, the DS-UWB system is subjected to " 8 " interfering WLAN channels ${ }^{5}$.

From these figures it can be seen that a good agreement with the simulation results in both cases is achieved, where we can conclude the validation of the derived analytical formula.

\footnotetext{
${ }^{5}$ The total interference power level is the same in both cases.
} 


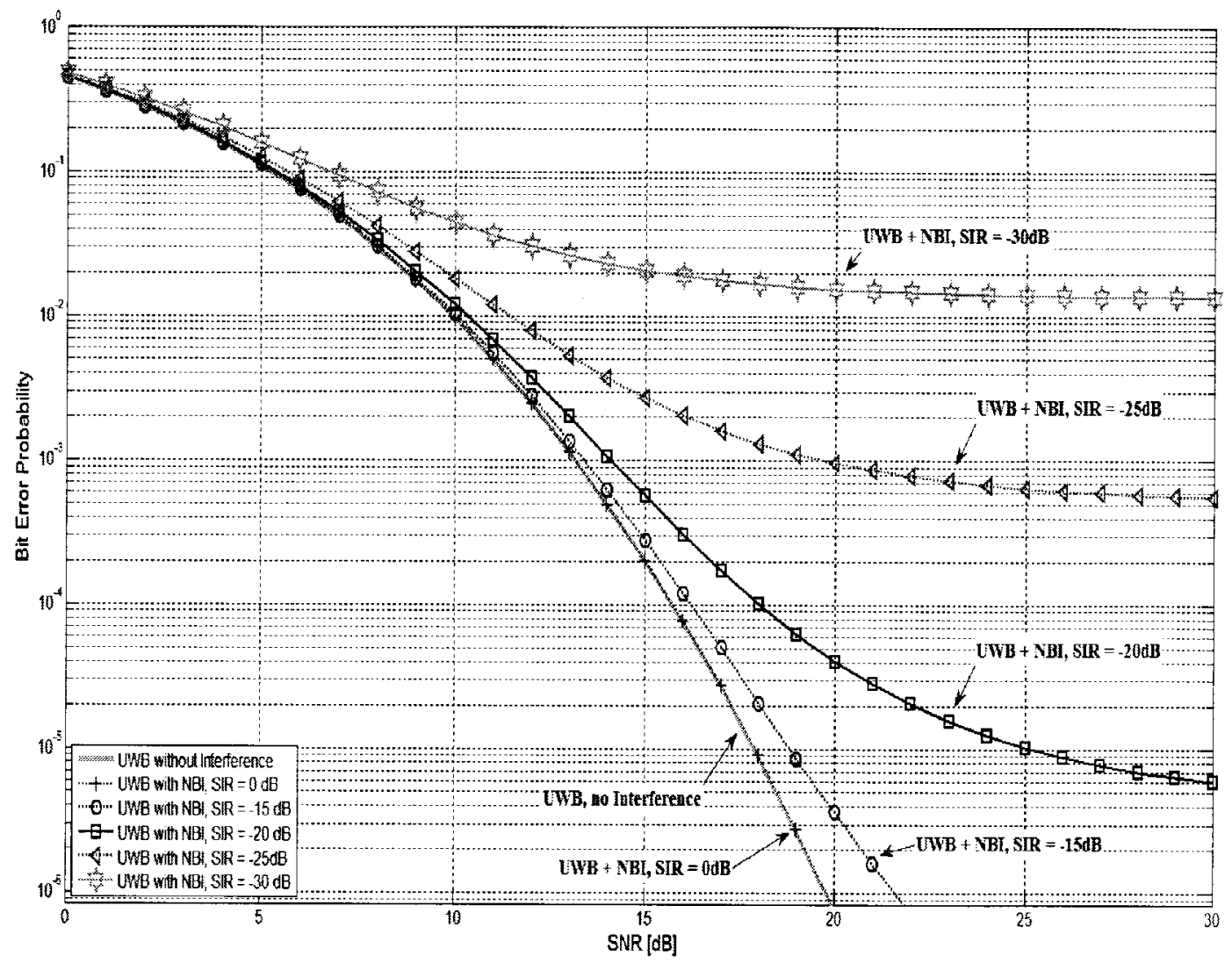

Figure 5.15: BER performance of the MRC-Rake receiver in presence of IEEE802.11a in the CM1 channel model, $\mathrm{L}=2, \mathrm{~K}=20$ for different SIRs.

Figure 5.19 depicts the effect of changing the number of the Rake receiver fingers on the performance of the UWB system in the CM1, and the CM4 channel models at $\mathrm{SIR}=-30 \mathrm{~dB}$. As expected it can be seen that, by increasing the number of the Rake fingers from 5 to 20 the SNR degradation is reduced by $4 \mathrm{~dB}$ and $6 \mathrm{~dB}$ in the CM1 and the CM4 channel models respectively at a $\mathrm{BER} \approx 6 \times 10^{-2}$ at the expense of increasing the system complexity. 


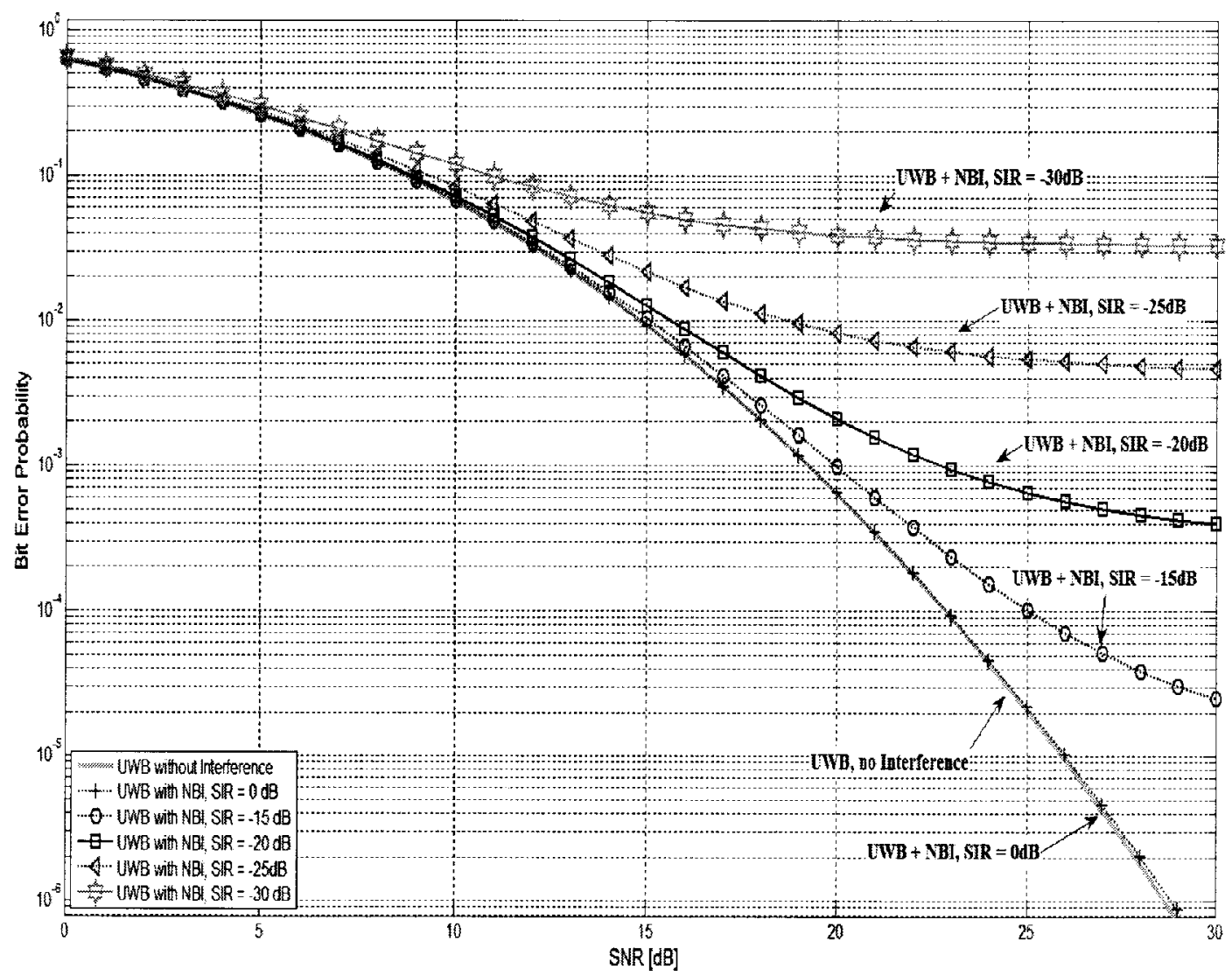

Figure 5.16: BER performance of the MRC-Rake receiver in presence of IEEE802.11a in the CM4 channel model, $\mathrm{L}=2, \mathrm{~K}=20$ for different SIRs. 


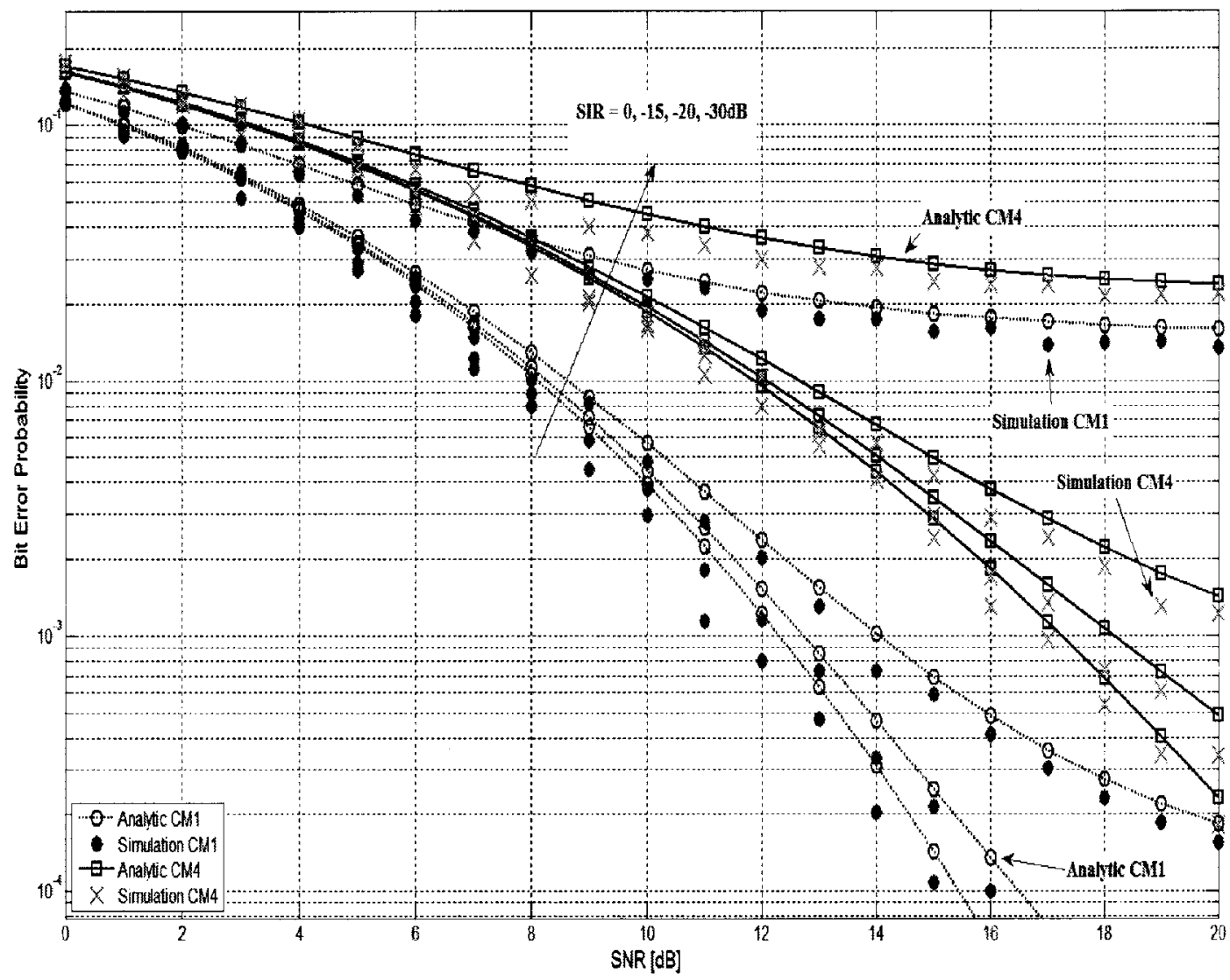

Figure 5.17: Simulation versus analytic evaluation of the BER performance of the MRC-Rake receiver in the presence of IEEE802.11a in the CM1 and the CM4 channel models. 


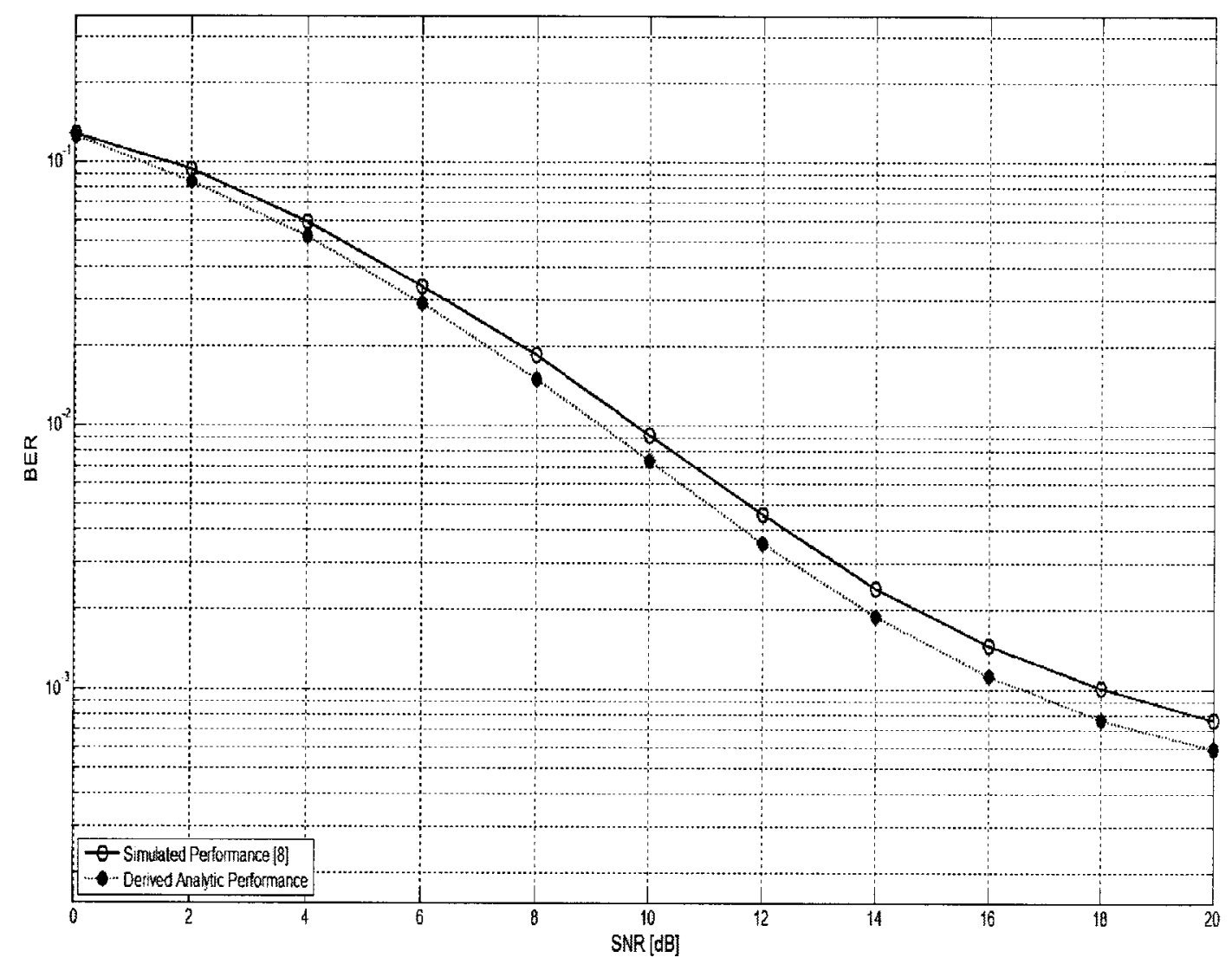

Figure 5.18: A comparison between the derived analytic performance presented in equation (5.84) (in presence of a single WLAN NBI signal) and the simulation experiment presented in [12] in the CM1 channel model in presence of " 8 " interfering WLAN channels and for 10-fingers MRC Rake receiver (the total interference power is the same in both cases). 


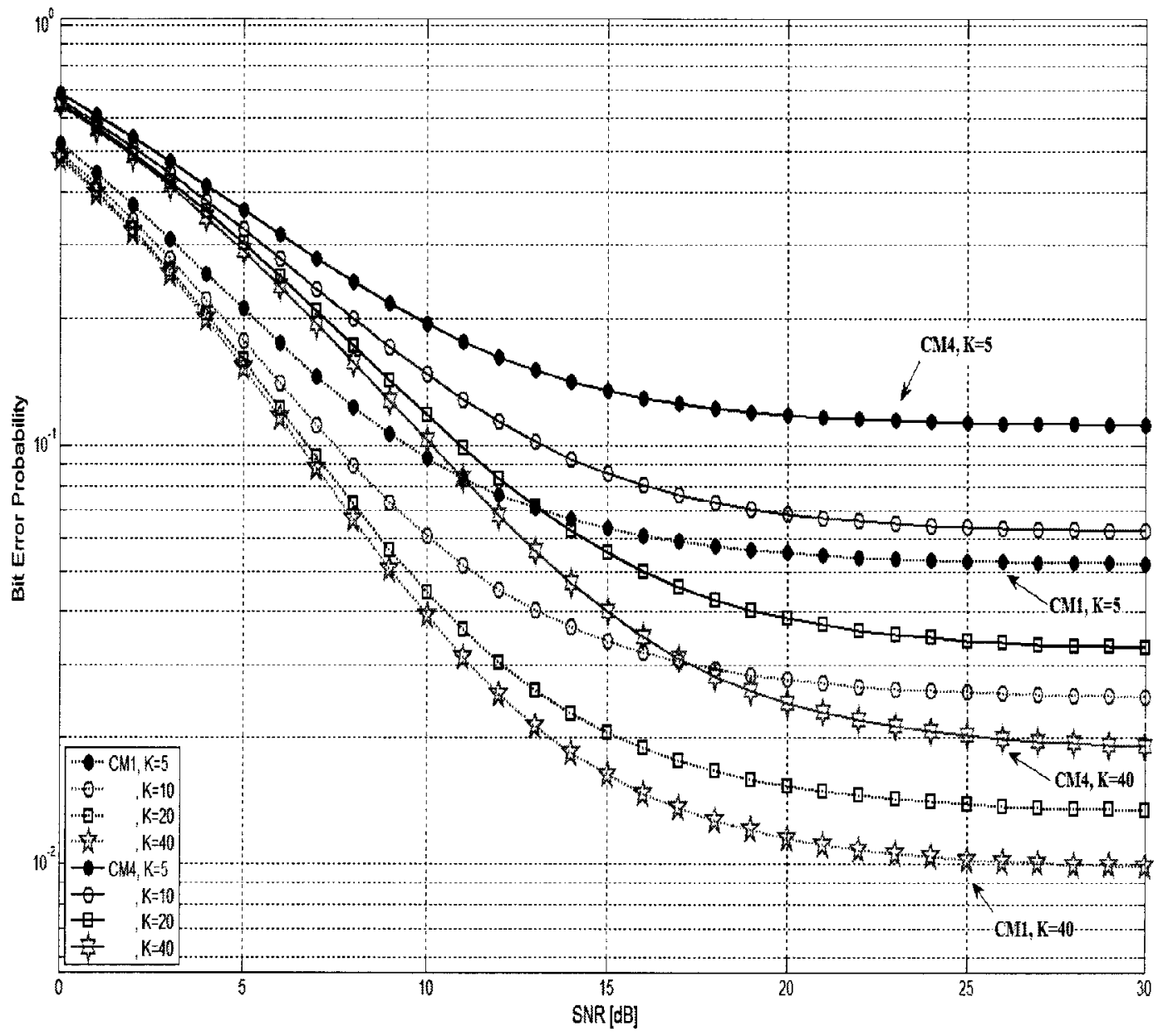

Figure 5.19: Impact of changing the number of Rake receiver fingers on the performance of the UWB system in the CM1 and the CM4 channel models, SIR = $-30 \mathrm{~dB}, \mathrm{~L}=2$. 


\subsection{Performance of the ICS in Various UWB Channel Models}

With the assumption of perfect channel estimation conditions for both the UWB and NBI signals, the performance of the ICS is evaluated with the aid of the previously presented formulas ${ }^{6}$ in various fading channel environments .

\subsubsection{ICS Performance in a Log-normal Flat Fading Channel}

The BER performance of the canceller scheme in a Log-normal flat fading channel can be evaluated as

$$
\begin{aligned}
\left(P_{e}\right)_{u w b}^{i c s}=\left(P_{e-u w b}^{W O I}\right)(1 & \left.-P_{e-o f d m}^{e=1}-P_{e-o f d m}^{e=2}-\ldots-P_{e-o f d m}^{e=e^{\prime}}\right) \\
& +\left(P_{e-u w b}^{1}\right)\left(P_{e-o f d m}^{e=1}\right)+\ldots+\left(P_{e-u w b}^{e^{\prime}}\right)\left(P_{e-o f d m}^{e=e^{\prime}}\right)
\end{aligned}
$$

where $P_{e-u w b}^{W O I}$ is the probability of the UWB system in a Log-normal flat fading channel in the absence of NBI, $P_{e-u w b}^{e^{\prime}}$ is the probability of the UWB system in the presence of $e^{\prime}$ tone interferers ${ }^{7}$ and $P_{e-o f d m}^{e=e^{\prime}}$ is the NBI receiver probability of symbol error for $e^{\prime}$ error symbols in a block of $\mathrm{N}$ symbols in a Rayleigh flat fading channel.

Figure 5.20 depicts the BER performance of the canceller scheme at SIR = $-10 \mathrm{~dB}$ and the $\mathrm{dB}$-spread value is $3 \mathrm{~dB}$. As presented in this figure, for an av. Pse equals to $1 \times 10^{-3}$, the canceller can perfectly suppress the impact of the IEEE $802.11 \mathrm{a}$ WLAN system interference on the UWB system. Whereas, for an av. Pse $=0.05$, the SNR degradation is reduced to be less than $2 \mathrm{~dB}$ at a $\mathrm{BER}=1 \times 10^{-3}$, where we can conclude the capability of the ICS to mitigate the impact of a NBI signal on the IR-UWB communication system in such fading channel environment.

\footnotetext{
${ }^{6}$ Formulas evaluate the BER performances of UWB system in various fading channel models.

7 It can be evaluated by using equation (5.58).
} 


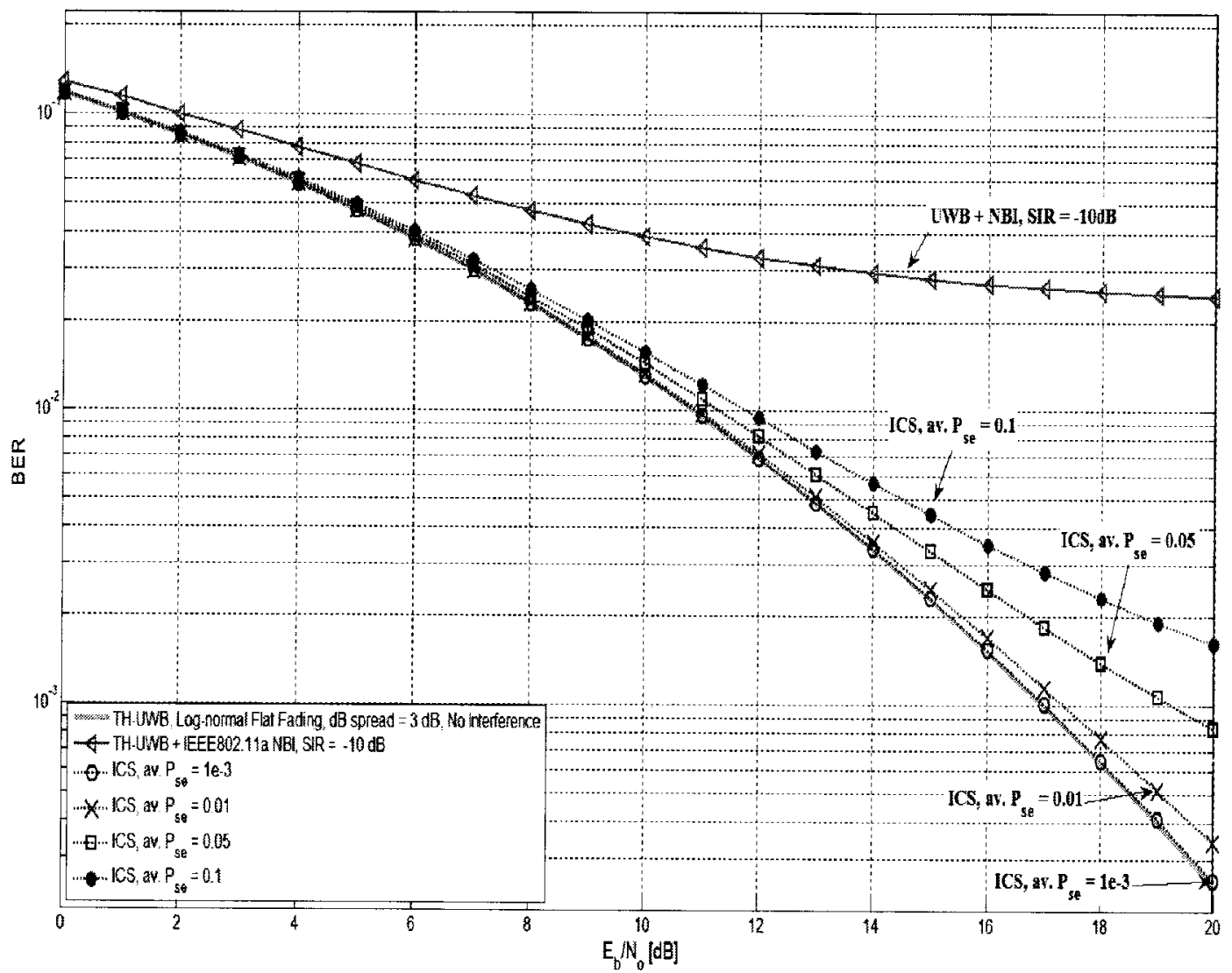

Figure 5.20: ICS BER performance in the presence of the IEEE802.11a NBI in a Log-normal flat fading channel, SIR $=-10 \mathrm{~dB}$. 


\subsubsection{ICS Performance in the IEEE802.15.3a UWB Channel Models}

The performance of the ICS in the IEEE802.15.3a UWB fading channel models in the presence of the standard IEEE802.11a NBI can be evaluated by the substitution of equation (5.84) into equation (5.86).

The terms in equation (5.86) can be defined as: $P_{e-u w b}^{W O I}$ is the probability of the DS-BPSK system in the absence of NBI, $P_{e-u w b}^{e^{\prime}}$ is the probability of the UWB system in the IEEE802.15.3a UWB fading channel models in the presence of $e^{\prime}$ tone interferers $^{8}$ and $P_{e-o f d m}^{e=e^{\prime}}$ is the NBI receiver probability of symbol error for $e^{\prime}$ error symbols in a block of length $\mathrm{N}$ symbols in a Rayleigh fading channel.

Figures 5.21 and 5.22 depict the BER performance of a DS-BPSK system with the aid of the ICS in the CM1 and the CM4 channel models respectively.

The used numerical values are: $T_{b}=16 \mathrm{~ns}$, and $\tau_{p}=0.192$ ns. Assuming $\beta_{o, o}=1$, $\sigma_{1}=\sigma_{2}=3.3941 \mathrm{~dB}$ and the number of receiving clusters $\mathrm{L}=2$ with the number of receiving rays within a cluster $\mathrm{K}=20$ at $\mathrm{SIR}=-20 \mathrm{~dB}$.

From these figures it can be seen that, due to the presence of NBI, the performance a DS-UWB system is severely degraded. However, with the use of the canceller scheme, the impact of interference can be completely suppressed at an av. Pse = $1 \times 10^{-3}$. For an av. Pse $=0.05$, the SNR degradation is expected to be less than 3 $\mathrm{dB}$ at $\mathrm{BER}=1 \times 10^{-3}$ in the CM1 channel model and less than $4 \mathrm{~dB}$ in the CM4 channel model at $\mathrm{BER}=2 \times 10^{-3}$.

\subsection{Comparison between the ICS Analytical and Simulation Results}

In this section, a comparison between the simulated performances of the ICS and the obtained analytical ones will be presented.

Figure 5.23 depicts this comparison in AWGN channel and some of the obtained

\footnotetext{
${ }^{8}$ It can be evaluated by using equation (5.84).
} 


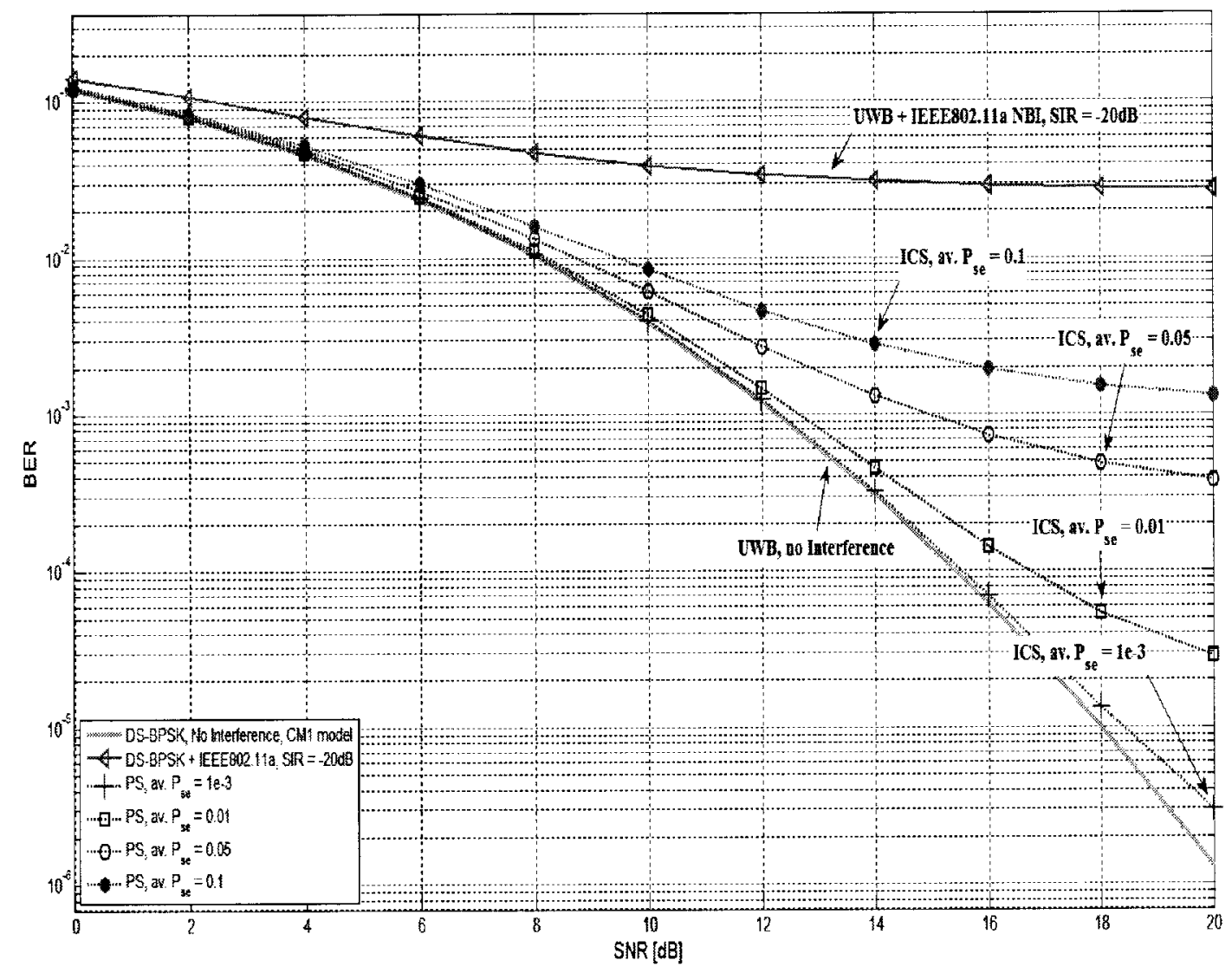

Figure 5.21: ICS BER performance in the CM1 channel model, SIR $=-20 \mathrm{~dB}$. 


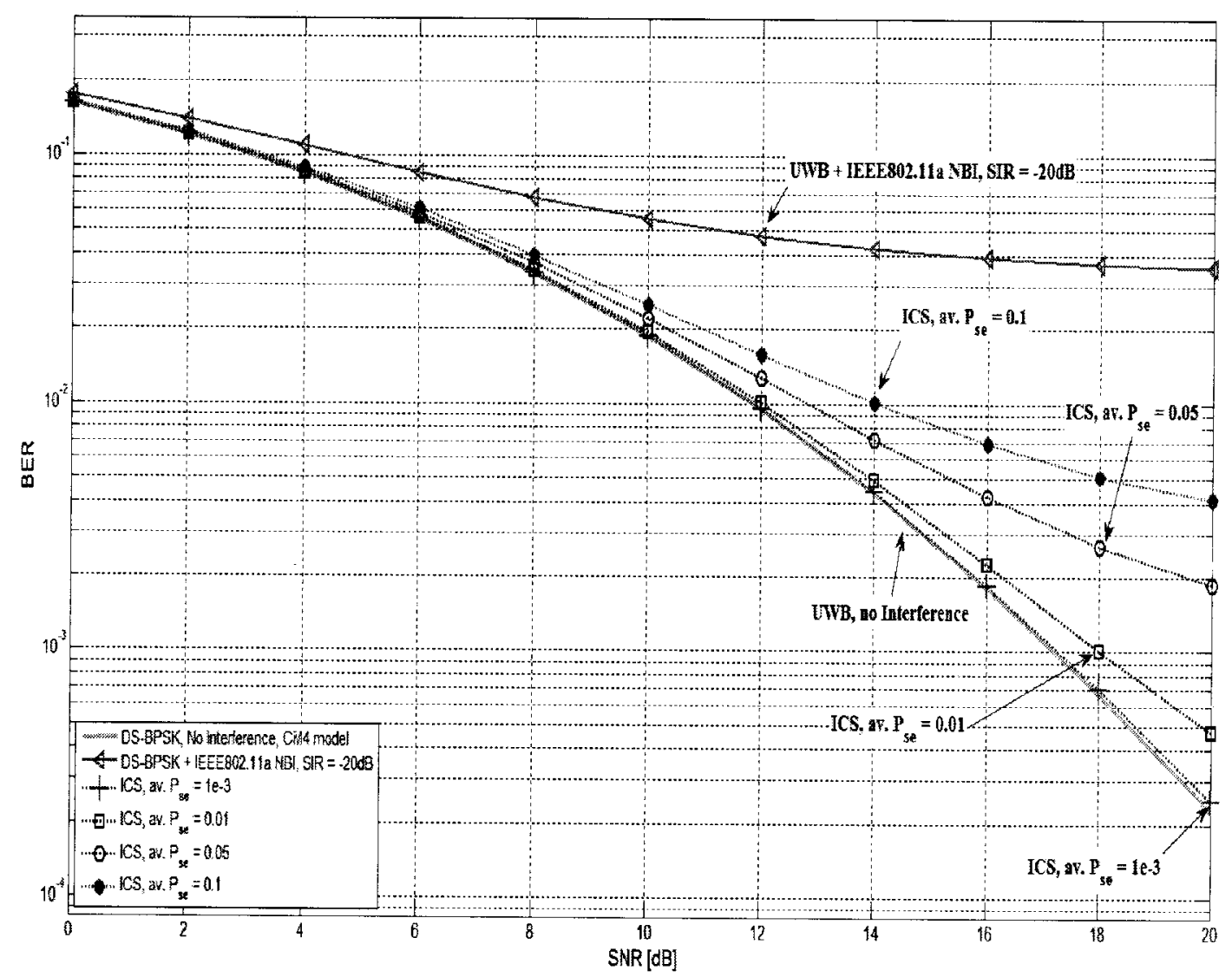

Figure 5.22: ICS BER performance in the $\mathrm{CM} 4$ channel model, $\mathrm{SIR}=-20 \mathrm{~dB}$. 
analytical results presented in chapter $3^{9}$, evaluated at $\mathrm{SIR}=-20 \mathrm{~dB}$.

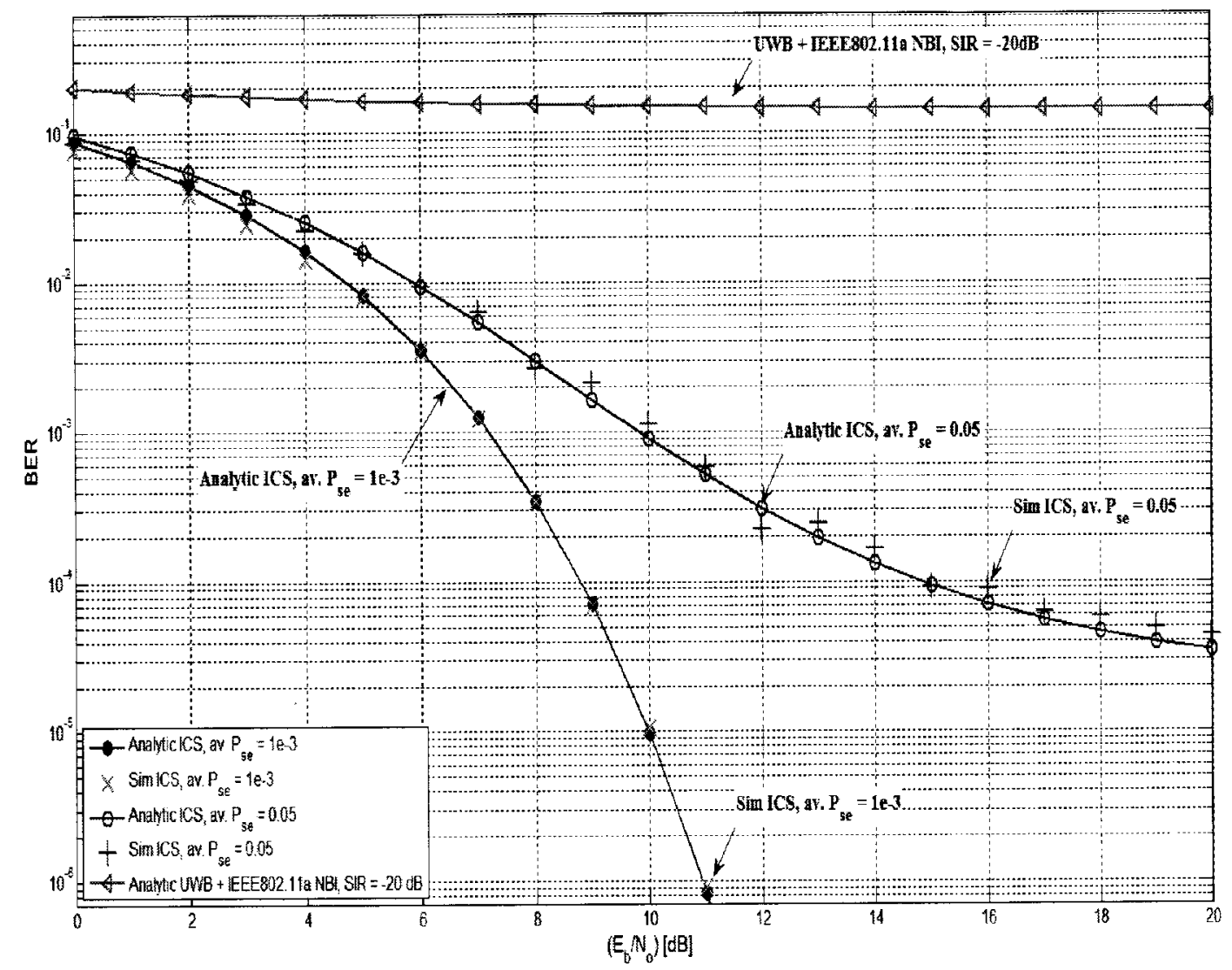

Figure 5.23: Simulation and analytical performance comparison of the ICS in AWGN channel, SIR $=-20 \mathrm{~dB}$.

From this figure it can be seen that, although the IEEE802.11a interference can severely deteriorate the performance of the TH-PPM system, the NBI impact on such victim systems can be completely suppressed with the aid of the ICS at an av. Pse equals to $1 \times 10^{-3}$. The SNR degradation is expected to be less than $1 \mathrm{~dB}$ and $5 \mathrm{~dB}$ at a $\mathrm{BER}=1 \times 10^{-4}$, for an av. Pse $=0.05$.

Figure 5.24 depicts the simulated performance of the ICS versus the analytical one in a Nakagami-m flat fading channel, for a Nakagami fading parameter $\mathrm{m}=2$ and $\mathrm{SIR}=-20 \mathrm{~dB}$.

\footnotetext{
${ }^{9}$ As presented in figure 3.12 .
} 


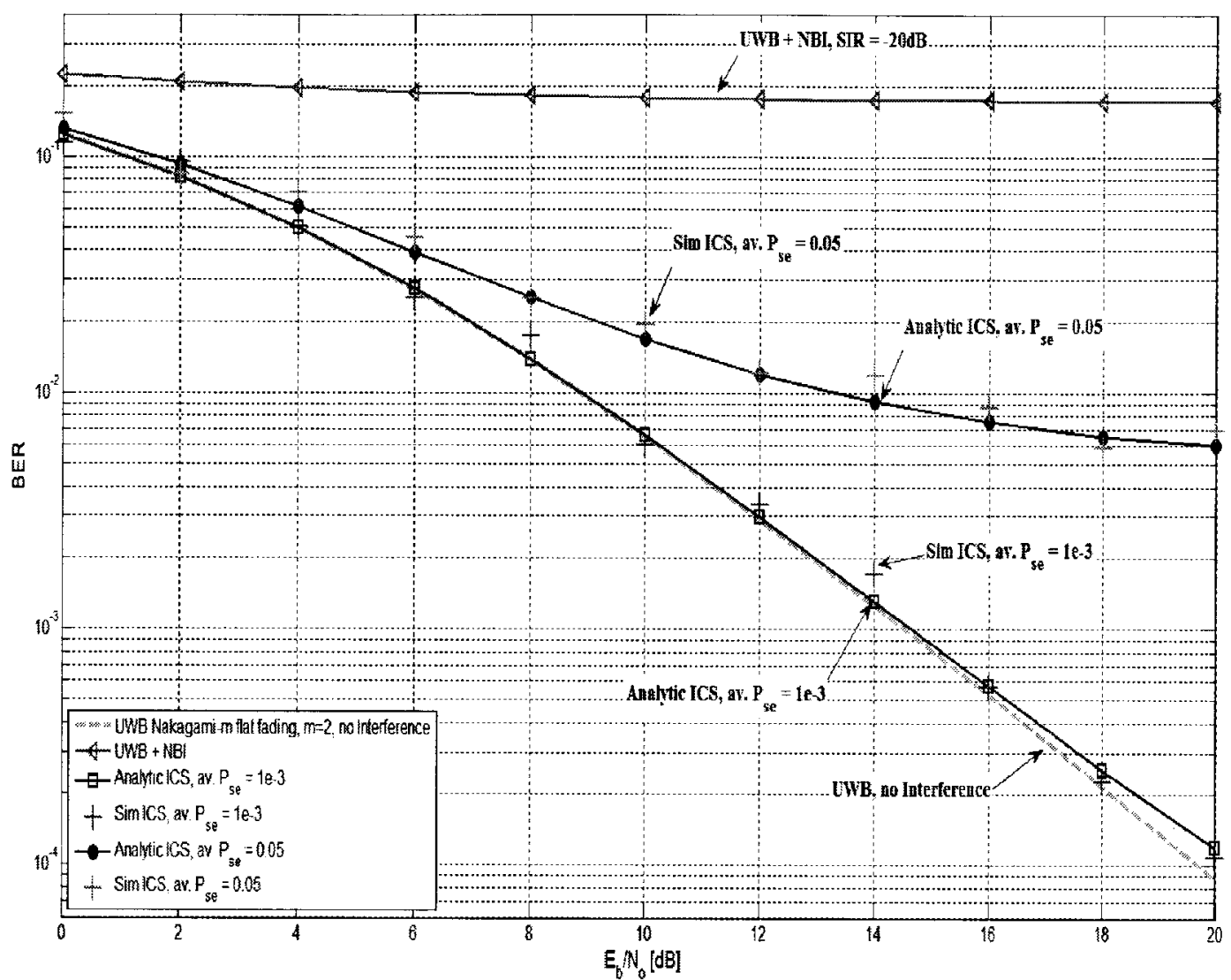

Figure 5.24: Simulation and analytical performance comparison of the ICS in a Nakagami-m flat fading channel, $\mathrm{m}=2$ and $\mathrm{SIR}=-20 \mathrm{~dB}$.

The simulation and analytical results of the ICS in a Log-normal flat fading channel is depicted in figure 5.25 at $\mathrm{SIR}=-10 \mathrm{~dB}$ and $\mathrm{dB}$-spread $=3 \mathrm{~dB}$.

From all these figures, it can be seen that the simulation and the analytical results are in a good agreement, in which we can conclude the validation of the obtained analytical results. 


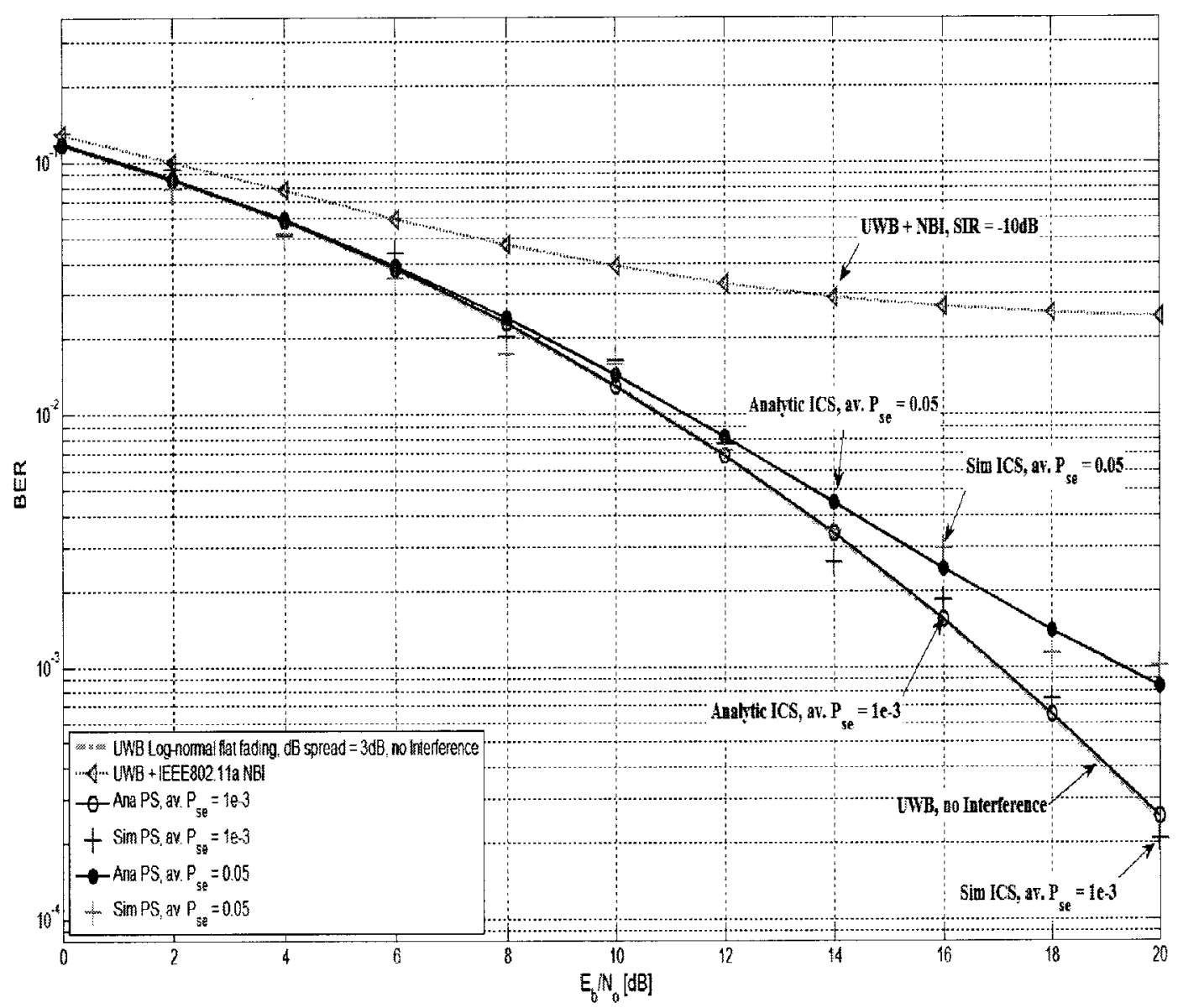

Figure 5.25: Simulation and analytical performance comparison of the ICS in a Log-normal flat fading channel, dB-spread $=3 \mathrm{~dB}$ and SIR $=-10 \mathrm{~dB}$. 


\section{Chapter 6}

\section{ICS Performance in Various Scenarios}

In this chapter, the performance of the ICS is investigated in different scenarios of operation such as: 1) the presence of multiple WLAN NBI signals. 2) The presence of symbol timing errors between the regenerated interference signal and the received NBI signal at the input of the subtraction process. 3) The presence of MAI. 4) A performance comparison with one of the used canceller schemes "notch filter".

\subsection{ICS Performance in the Presence of Multiple NBI Signals}

The capability of the canceller scheme to mitigate the impact of two WLAN NBI signals is investigated. Figure 6.1 depicts a schematic diagram of the ICS in the presence of two IEEE802.11a WLAN NBI signals. The ICS attempts to demodulate and to regenerate each NBI signal individually. The regenerated IEEE802.11a NBI signals are subtracted from the received signal at the input of the UWB receiver.

The WLAN NBI signals were simulated with center frequencies $5.22 \mathrm{GHz}$ and 5.745 GHz. The data rate of the UWB system is $100 \mathrm{Mbps}$.

Figure 6.2 depicts the impact of the presence of one and two NBI signals on the performance of a TH-PPM system in AWGN channel in addition to the ICS BER performance in both cases evaluated at an av. Pse $=0.1$ and $\mathrm{SIR}=-15 \mathrm{~dB}$.

It can be seen that, an additional SNR degradation in the BER performance of the ICS, due to the presence of two NBI signals, is expected to reach up to $1 \mathrm{~dB}$ at BER $=1 \times 10^{-2}$. 


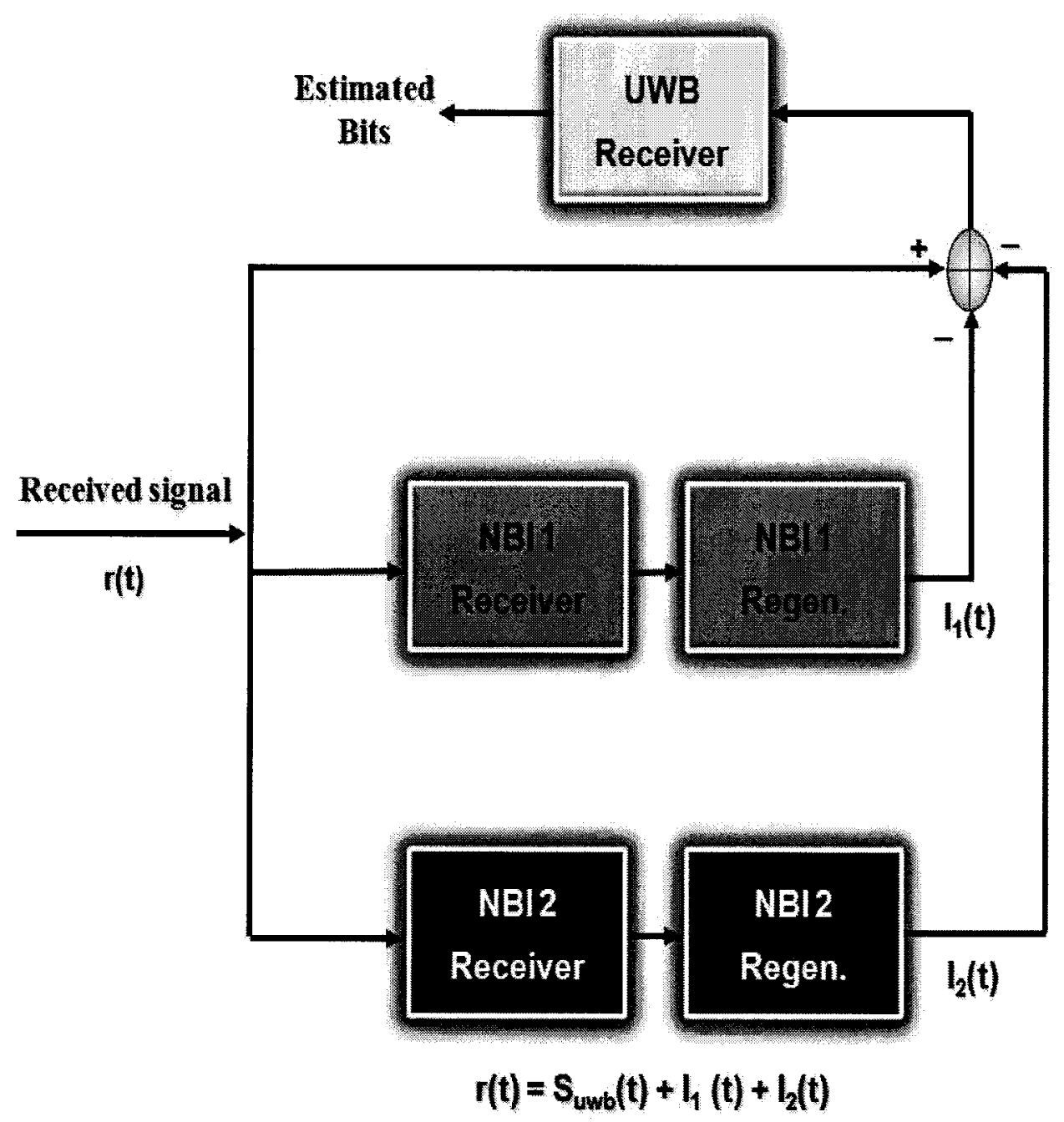

Figure 6.1: The ICS in the presence of two NBI signals. 


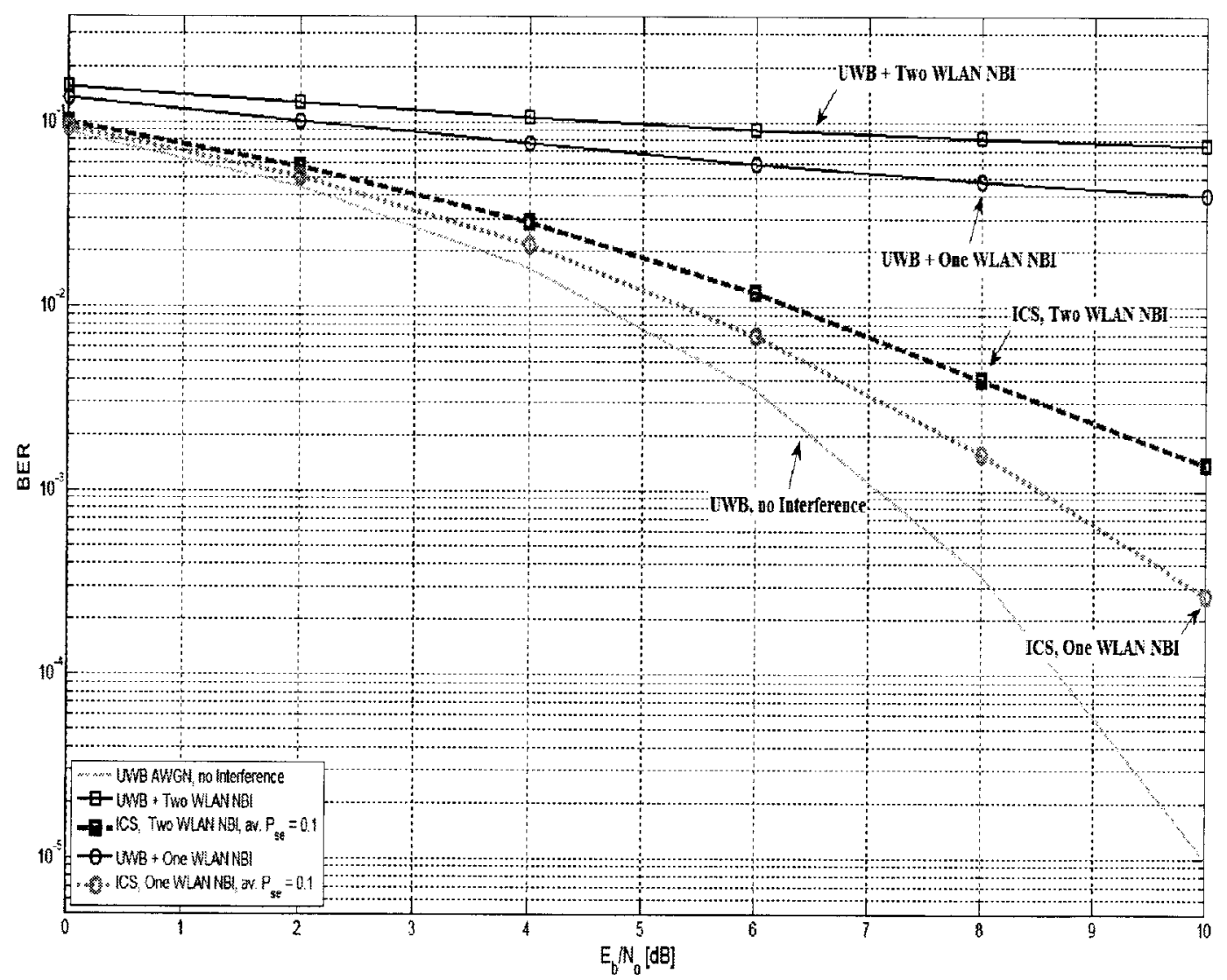

Figure 6.2: BER performance of the ICS in the presence of one and two NBI WLAN signals in AWGN channel, SIR $=-15 \mathrm{~dB}$. 
Figure 6.3 depicts a comparison between the BER performances of the canceller scheme in a Log-normal flat fading channel in the presence of one and two NBI WLANs, the $\mathrm{dB}$-spread value is $3 \mathrm{~dB}$ and the $\mathrm{SIR}=-10 \mathrm{~dB}$.

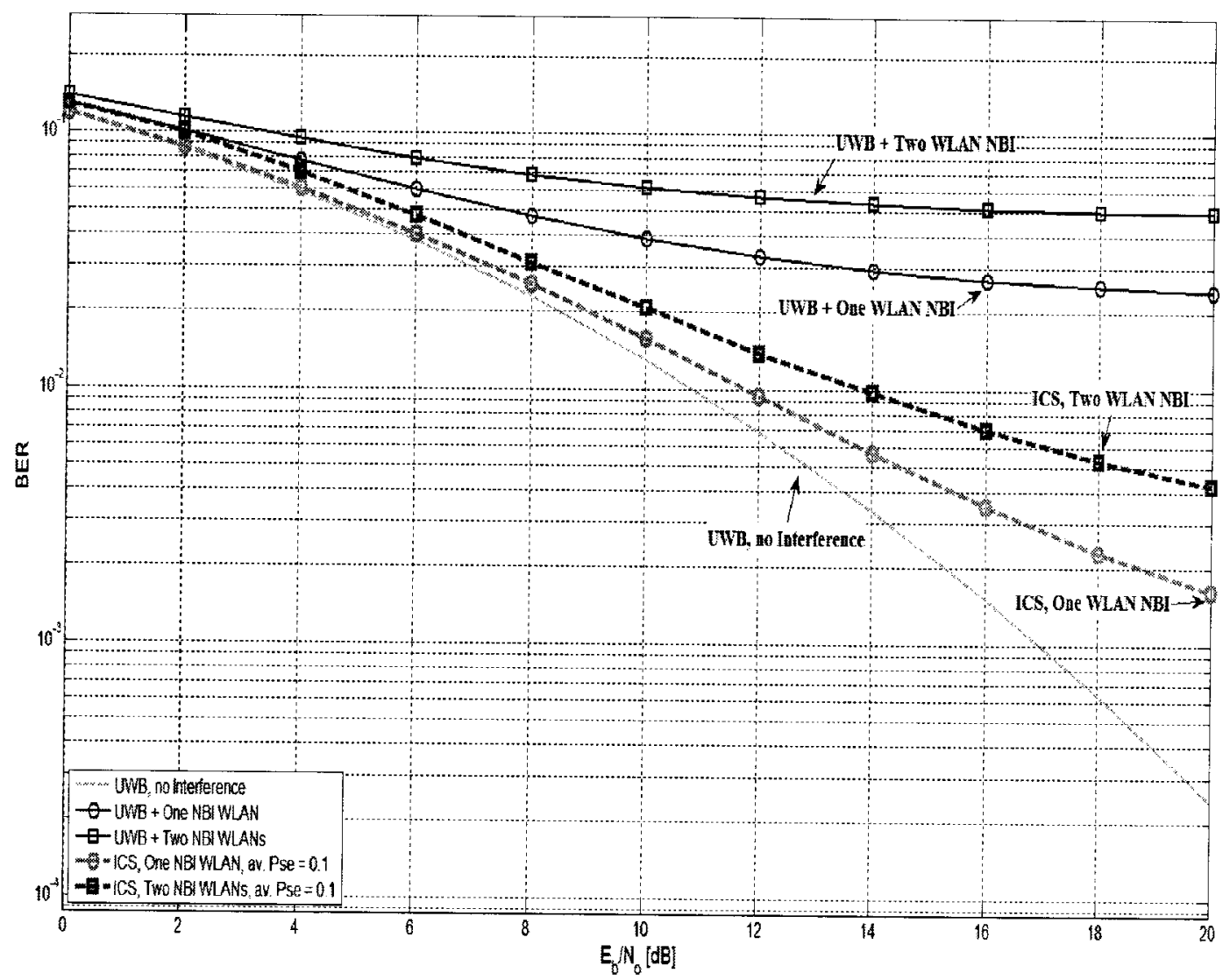

Figure 6.3: BER performance of the ICS in the presence of two NBI WLANs in a Log-normal flat fading channel, SIR $=-10 \mathrm{~dB}$.

It can be seen that, an additional SNR degradation in the ICS performance is expected to be less than $2 \mathrm{~dB}$ at $\mathrm{BER}=1 \times 10^{-2}$.

Figures 6.4 and 6.5 depict the BER performance of the ICS in the CM1 and the CM4 channel models respectively.

It has been shown that, in the CM1 channel model, there is an additional SNR degradation in the ICS BER performance equals to $2 \mathrm{~dB}$ at BER $=1 \times 10^{-4}$. Whereas, in the CM4 channel model, the additional SNR degradation is expected to be less than $3 \mathrm{~dB}$ at $\mathrm{BER}=1 \times 10^{-3}$. 


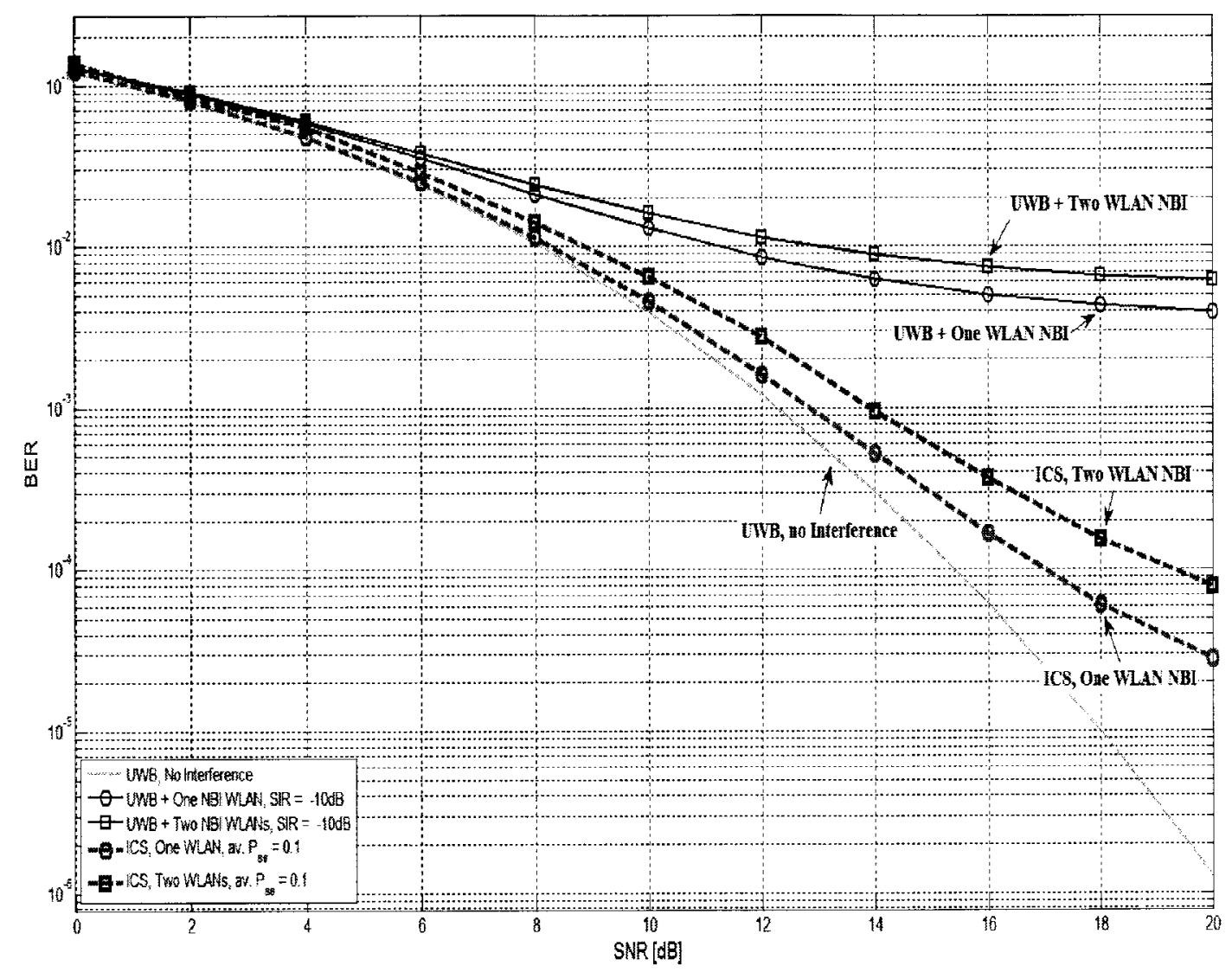

Figure 6.4: ICS BER performance in the presence of one and two NBI WLANs in the CM1 channel model.

\subsection{ICS Performance in the Presence of Symbol Timing Error}

In this section, the BER performance of the canceller scheme is investigated in the presence of symbol timing error (timing misalignment error) between the regenerated NBI signal and the received NBI signal at the input of the subtraction process.

The NBI signal simulated frame format was depicted in figure 4.10, where there exists a pilot symbol in the beginning of each frame in order to estimate the NBI channel. The NBI simulated parameters can be represented as follows:

The sampling frequency is $20 \mathrm{MHz}^{1}$, the symbol period ( $4 \mu \mathrm{s} \equiv 80$ samples) and the

1 The UWB sampling frequency is $50 \mathrm{GHz}$. 


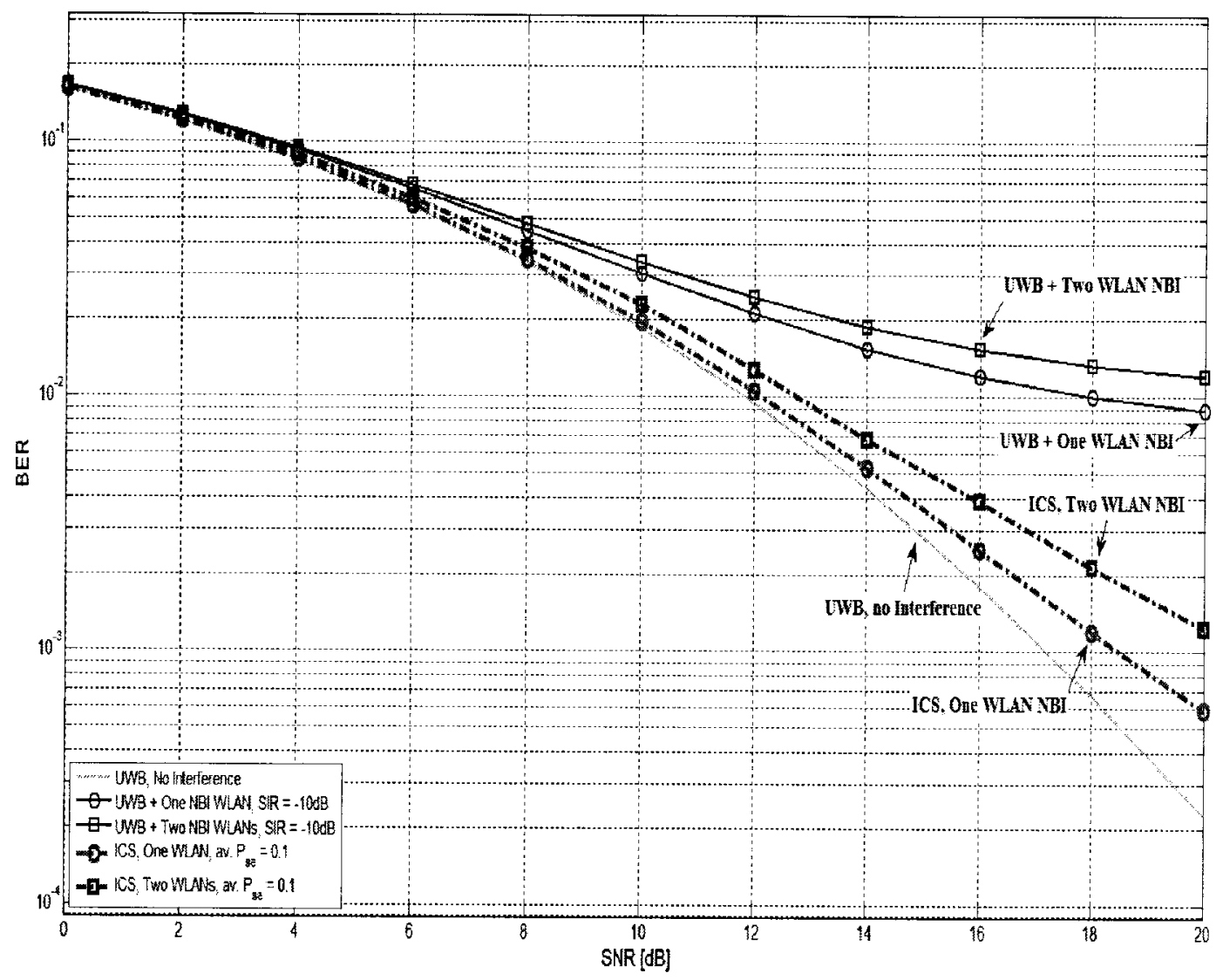

Figure 6.5: ICS BER performance in the presence of one and two NBI WLANs in the CM4 channel model.

duration of the GI is $800 \mathrm{~ns} \equiv 16$ samples. The useful symbol period is $3.2 \mu \mathrm{s} \equiv 64$ samples.

Figure 6.6 depicts the BER performance of the ICS in AWGN channel when: 1) the misalignment equals to 1000 samples $(0.5 \%)^{2}$. 2) the misalignment equals to 2000 samples (1\%). It can be seen that, for the $0.5 \%$ misalignment and $\mathrm{SIR}=-15 \mathrm{~dB}$, an additional SNR is expected to reach up to $2 \mathrm{~dB}$ at $\mathrm{BER}=1 \times 10^{-3}$. Whereas for the $1 \%$ misalignment, the additional SNR is expected to reach up to $3.5 \mathrm{~dB}$ at BER $=2 \times 10^{-2}$.

Figure 6.7 depicts the ICS performance in a Log-normal flat fading channel, the av. Pse $=0.1, \mathrm{~dB}$-spread value $=2 \mathrm{~dB}$ and $\mathrm{SIR}=-15 \mathrm{~dB}$. It can be seen that for

\footnotetext{
2 The timing error is measured with respect to the NBI symbol duration.
} 


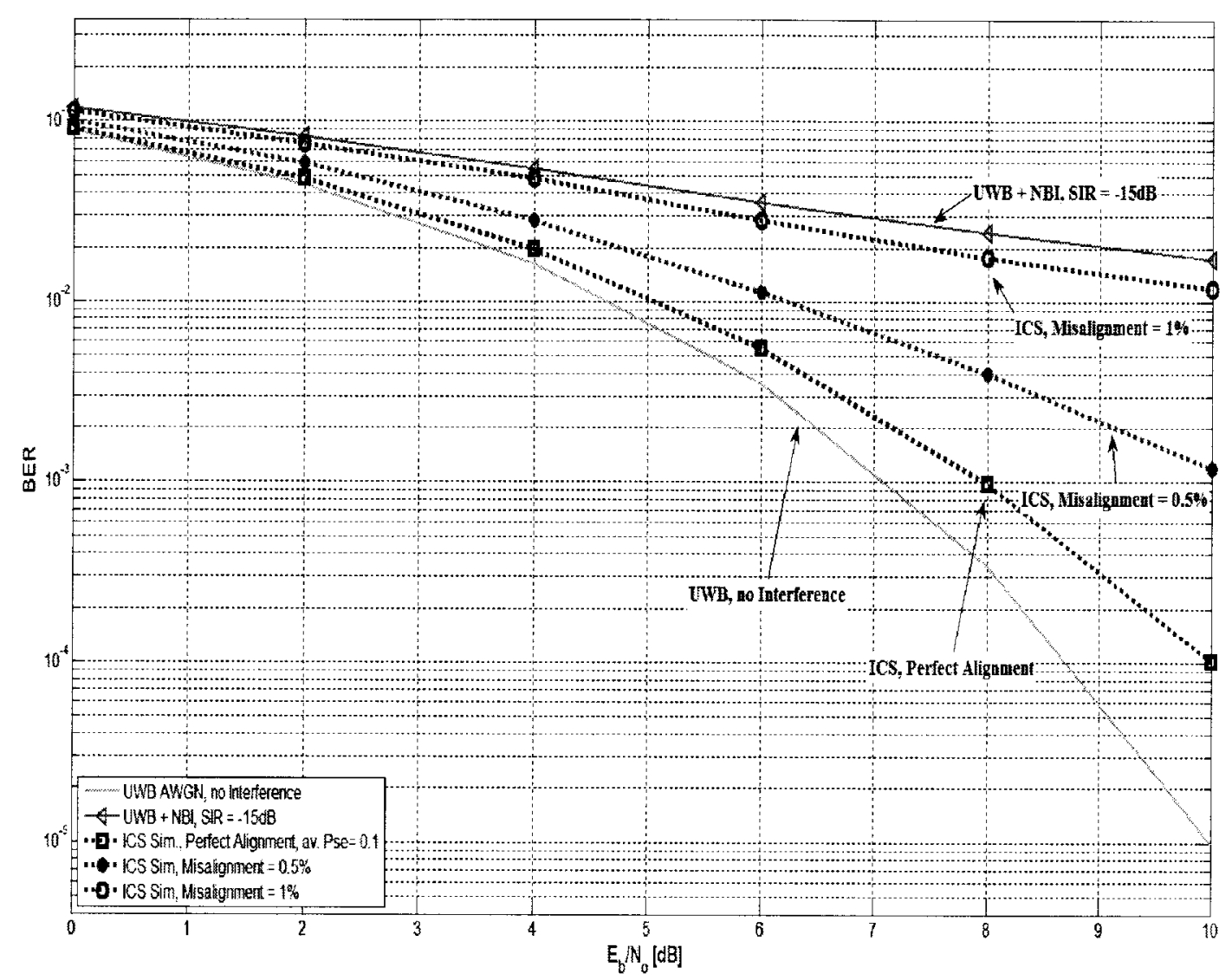

Figure 6.6: BER performance of the canceller scheme in the presence of symbol timing error in AWGN channel.

a timing misalignment error equals to $0.5 \%$, there is an additional SNR degradation equals to $2 \mathrm{~dB}$ at $\mathrm{BER}=3 \times 10^{-3}$, whereas, for a timing misalignment error equals to $1 \%$ there is an additional SNR degradation equals to $4 \mathrm{~dB}$ at BER $=5 \times 10^{-2}$.

Figures 6.8 and 6.9 depict this impact in the CM1 and the CM4 channel models respectively for a SIR $=-20 \mathrm{~dB}$. It can be seen that for a timing misalignment error equals to $0.5 \%$ at $\mathrm{BER}=1 \times 10^{-2}$, there are $3 \mathrm{~dB}$ and $4 \mathrm{~dB}$ additional SNR degradation at an av. Pse $=0.1$ for the $\mathrm{CM} 1$ and the $\mathrm{CM} 4$ channel models respectively. For a timing misalignment error equals to $1 \%$ at $\mathrm{BER}=2 \times 10^{-2}$, there are $4 \mathrm{~dB}$ and $8 \mathrm{~dB}$ additional SNR degradation in the CM1 and the CM4 channel models respectively. 


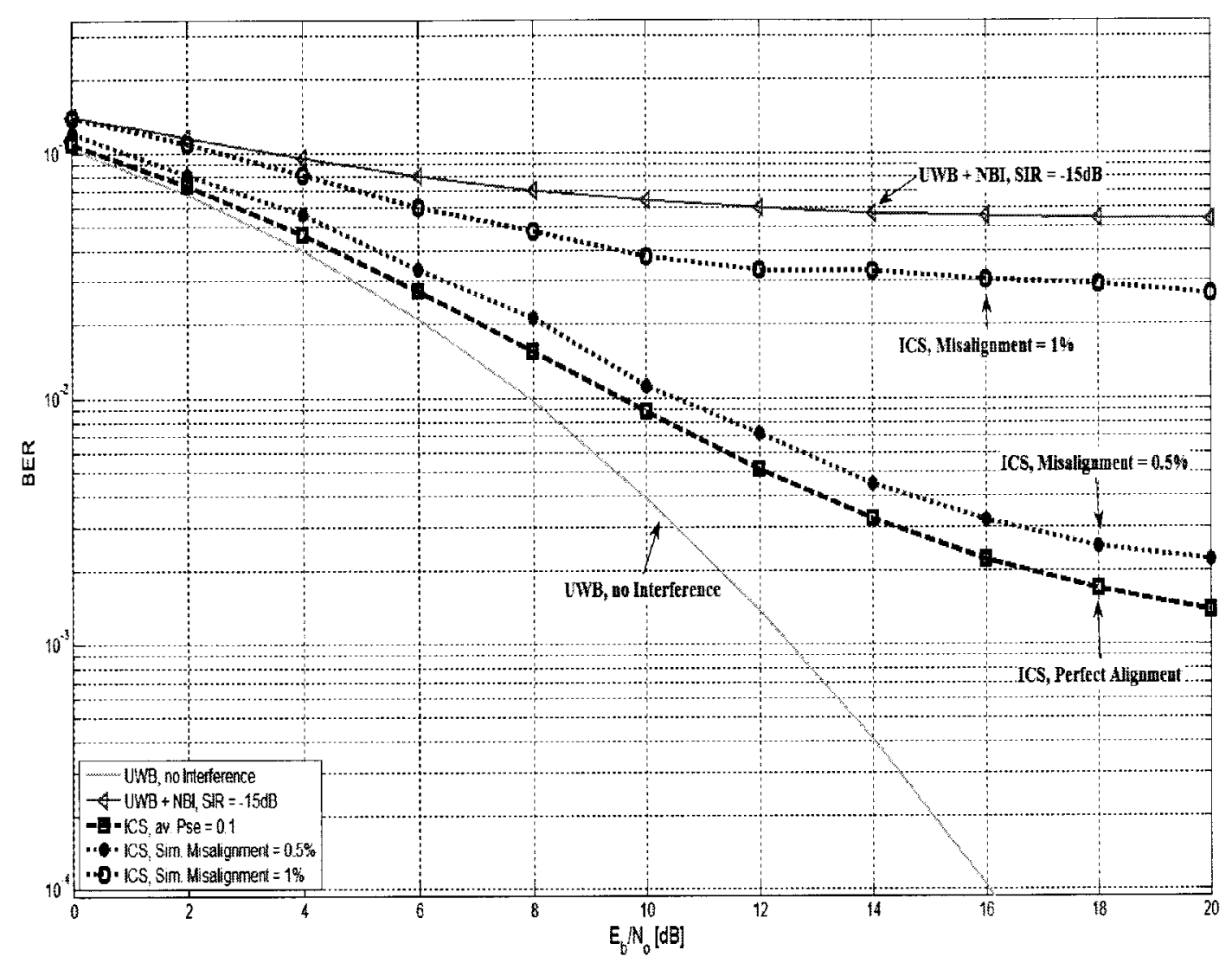

Figure 6.7: Timing error impact in a Log-normal flat fading channel, dB-spread $=$ $2 \mathrm{~dB}$ and $\mathrm{SIR}=-15 \mathrm{~dB}$.

\subsection{ICS Performance in the Presence of MAI}

As stated in chapter 2, a multiple access communication system is the system which capable of providing differcnt users the ability to share the same physical medium for transmitting and receiving different data flows. In the next subsection, we will describe the ICS performance in AWGN channel in the presence of both MAI and NBI signals for two scenarios: perfect and imperfect power control. 


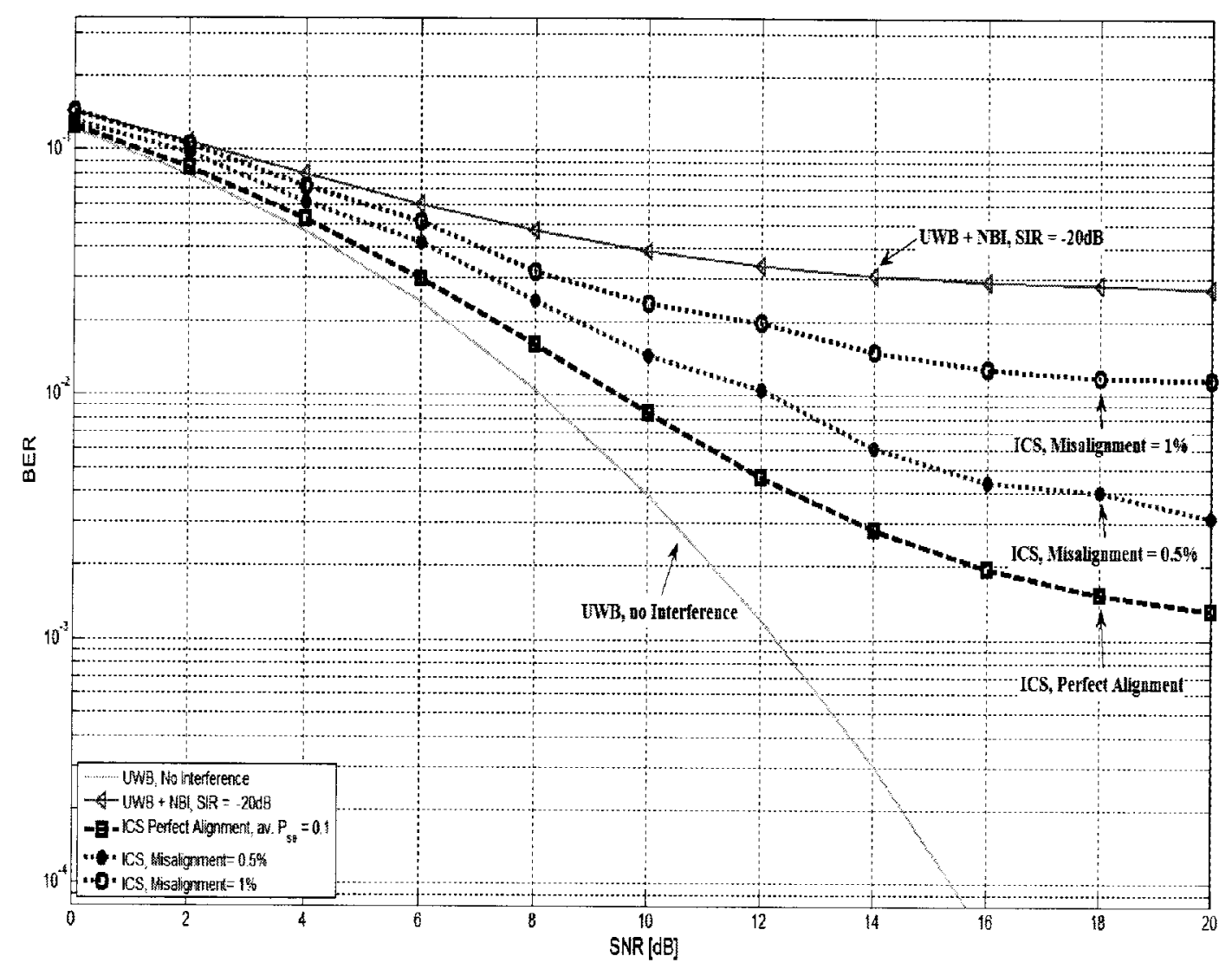

Figure 6.8: Symbol Timing error in the CM1 channel model.

\subsubsection{AWGN Channel Environment}

\subsubsection{Perfect Power Control}

Based on the SGA hypothesis and assuming that the desired user is the first user, the BER performance of a TH-PPM system in the presence of MAI in AWGN channel is given by [9]

$$
P_{e}=Q(\sqrt{\eta})
$$

where $\eta$ is the signal to interference plus noise ratio. $\eta$ can be written as

$$
\eta=\left[\left(\frac{E_{b}^{(1)}}{N_{o}}(1-\varrho)\right)^{-1}+\left(\frac{(1-\varrho)^{2} \cdot \gamma_{R}}{\sigma_{M}^{2} \cdot R_{b} \cdot \sum_{n=2}^{N_{u}} \frac{E_{R X}^{(n)}}{E_{R X}^{(1)}}}\right)^{-1}\right]^{-1}
$$




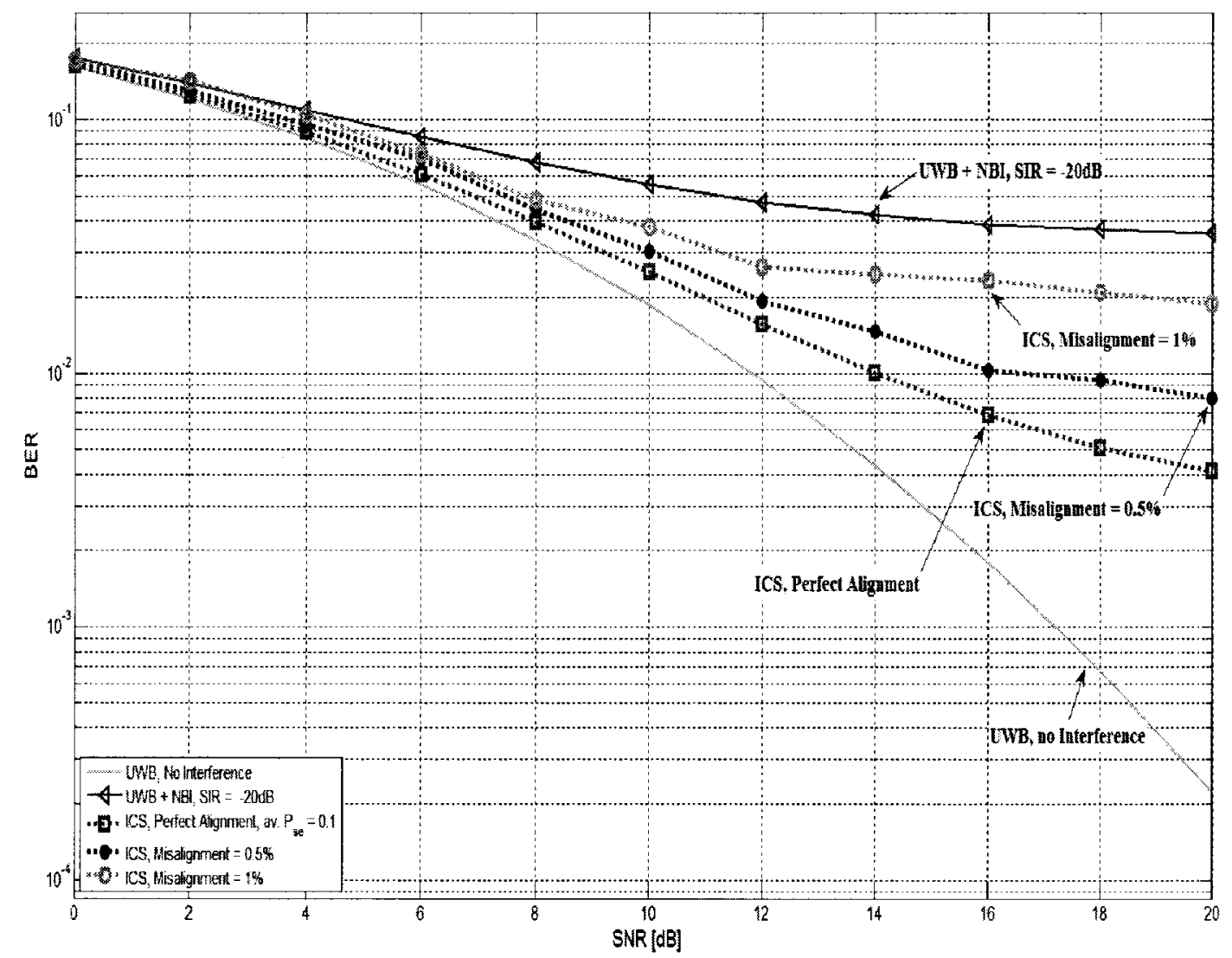

Figure 6.9: Symbol Timing error in the CM4 channel model. 
$E_{R X}^{(1)}$ and $E_{R X}^{(n)}$ are the received energies for the desired user and the $n^{\text {th }}$ interfering user respectively. Under the simplifying hypothesis of perfect power control, for example, that is, all terms $E_{R X}^{(i)}$ are equal for $\mathrm{i}=1, \ldots, N_{u}$. The relation between these two terms can be described as:

$$
\sum_{n=2}^{N_{u}} \frac{E_{R X}^{(n)}}{E_{R X}^{(1)}}=N_{u}-1
$$

where $N_{u}$ is the total number of transmitting users, $\varrho$ is the correlation coefficient between the TH-PPM transmitted pulses presented in equation (3.4), $\gamma_{R}=\frac{N_{s} T_{f}}{T_{b}}$, $\gamma_{R} \leq 1$, and $\sigma_{M}^{2}$ is given by [9]

$$
\sigma_{M}^{2}=\int_{0}^{T_{f}}\left(\int_{0}^{2 T_{m}} p(t-\tau) \cdot m(t) d t\right)^{2} d \tau
$$

where $m(t)$ is the UWB correlation mask.

In the case of orthogonal pulses, $\sigma_{M}^{2}$ can be simplified as

$$
\sigma_{M}^{2}=2 \int_{-T_{m}}^{T_{m}} R_{o}^{2}(\tau) d \tau
$$

$R_{o}(\tau)$ is the autocorrelation function of the UWB signal defined by

$$
R_{o}(\tau)=\int_{0}^{T_{m}} p(t-\tau) \cdot p(t) d t
$$

Figure 6.10 depicts the performance of a TH-PPM system in the presence of MAI for different number of users $\left(N_{u}=5,10\right.$ and 20 users). Assuming a six derivative Gaussian pulse is used with the following parameters: $T_{m}=0.5 \mathrm{~ns}, \tau_{p}=0.192 \mathrm{~ns}, \delta$ $=0.068 \mathrm{~ns}$ and $R_{b}=20 \mathrm{Mbps}$.

The SNR degradation, due to MAI, is expected to reach up to $4 \mathrm{~dB}$ at a $\mathrm{BER}=$ $1 \times 10^{-4}$.

The performance of a UWB system in the presence of both NBI and MAI signals is evaluated. The NBI is modeled as the standard IEEE802.11a signal, and as presented in chapter 3 , it can be approximated as sum of $N_{i}$ tone interferers, with 


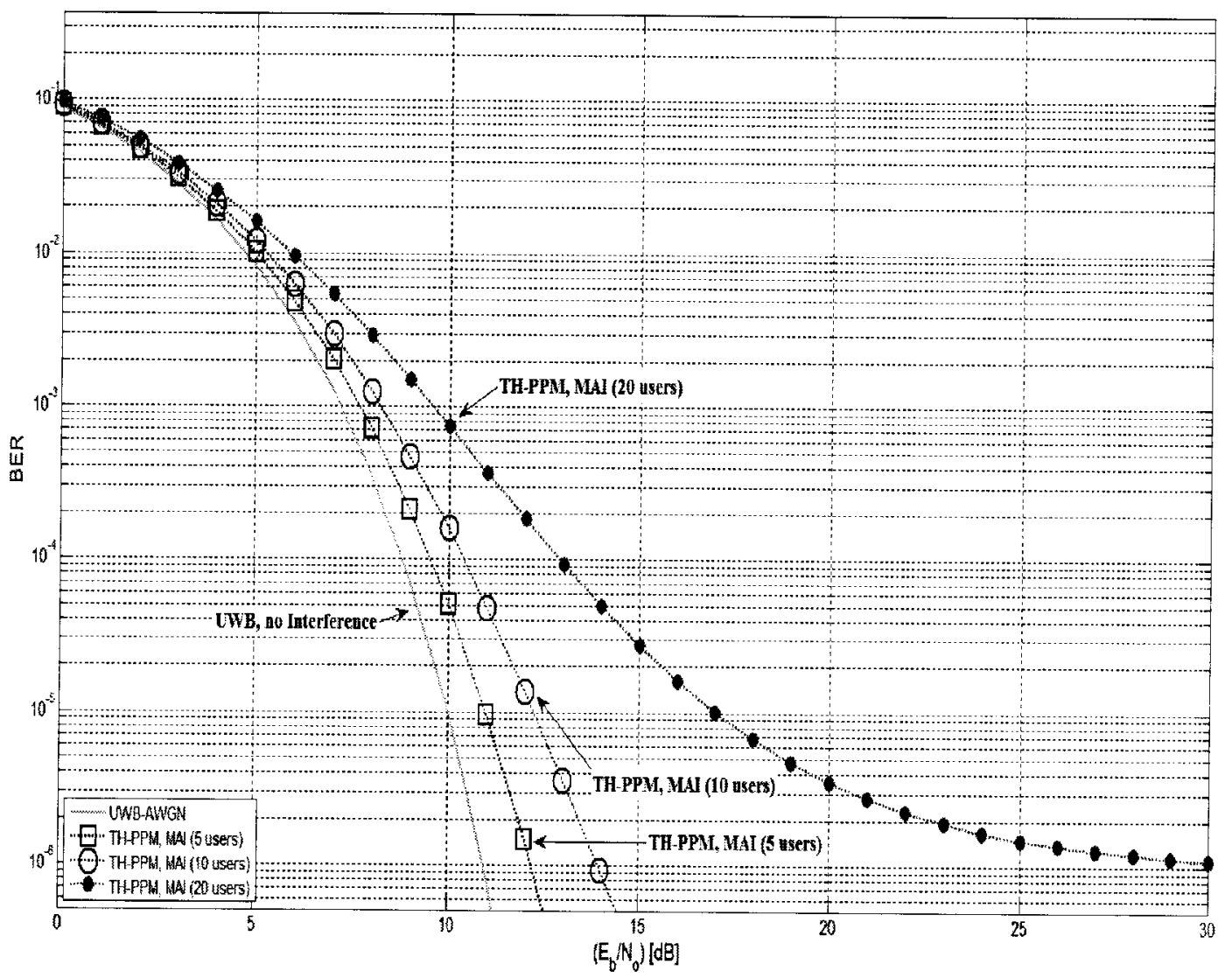

Figure 6.10: BER performance of a TH-PPM system in the presence of MAI 
variance

$$
\sigma_{i}^{2}=\frac{I}{N_{i}} \sum_{n=1}^{N_{i}}\left|M\left(f_{n}\right)\right|^{2}
$$

The NBI SIR term can be defined as

$$
S I R_{N B I}=\frac{\sum_{n=1}^{N_{i}}\left|M\left(f_{n}\right)\right|^{2}}{S I R \cdot N_{i} \cdot(1-\varrho)^{2}}
$$

In the presence of NBI and MAI signals, $\eta$ can be rewritten as

$$
\eta=\left[\left(\frac{E_{b}^{(1)}}{N_{o}}(1-\varrho)\right)^{-1}+\left(\frac{(1-\varrho)^{2} \cdot \gamma_{R}}{\sigma_{M}^{2} \cdot R_{b} \cdot \sum_{n=2}^{N_{u}} \frac{E_{R X}^{(n)}}{E_{R X}^{(1)}}}\right)^{-1}+\left(\frac{S I R \cdot N_{i} \cdot(1-\varrho)^{2}}{\sum_{n=1}^{N_{i}}\left|M\left(f_{n}\right)\right|^{2}}\right)^{-1}\right]^{-1}
$$

Figure 6.11 depicts the impact of both the NBI signal and MAI on the performance of a TH-PPM system for SIR $=-15$ and $-20 \mathrm{~dB}$ and for different number of users 5 , 10 and 20 users.

It can be seen that in the presence of 20 users and SIR $=-15 \mathrm{~dB}$, an additional SNR degradation, due to NBI, is expected to reach up to $5 \mathrm{~dB}$ at a $\mathrm{BER}=2 \times 10^{-3}$.

The BER performance of the ICS in the presence of both the MAI and NBI can be written as

$$
\begin{aligned}
&\left(P_{e}\right)_{U W B}^{i c s}=\left(P_{e-u w b}^{A W G N}\right)\left(1-P_{e-o f d m}^{e=1}-P_{e-o f d m}^{e=2}-\ldots-P_{e-o f d m}^{e=e^{\prime}}\right) \\
&+\left(P_{e-u w b}^{1}\right)\left(P_{e-o f d m}^{e=1}\right)+\left(P_{e-u w b}^{2}\right)\left(P_{e-o f d m}^{e=2}\right)+\ldots \\
&+\left(P_{e-u w b}^{e^{\prime}}\right)\left(P_{e-o f d m}^{e=e^{\prime}}\right)
\end{aligned}
$$

where $P_{e-u w b}^{A W G N}$ is the BER performance of a UWB system in AWGN channel in the absence of NBI and the presence of $\mathrm{MAI}^{3}, P_{e-o f d m}^{e=1}, \ldots, P_{e-o f d m}^{e=e^{\prime}}$ are the NBI receiver probabilities of OFDM symbol error when there are one, ..., and $e^{\prime}$ symbol errors occurred in a block of length $\mathrm{N}$ respectively ${ }^{4}$ and $P_{e-u w b}^{1}, \ldots, P_{e-u w b}^{e^{\prime}}$ are the BER performances of a UWB system in the presence of a single, ..., and $e^{\prime}$ tone interferers respectively along with the presence of $\mathrm{MAI}^{5}$.

\footnotetext{
${ }^{3}$ It could be evaluated using equations (6.1 and 6.2).

${ }^{4}$ It could be evaluated using equations ( 3.6 and 3.7 ).

5 It could be evaluated using equations ( 6.8 and 6.9 ).
} 


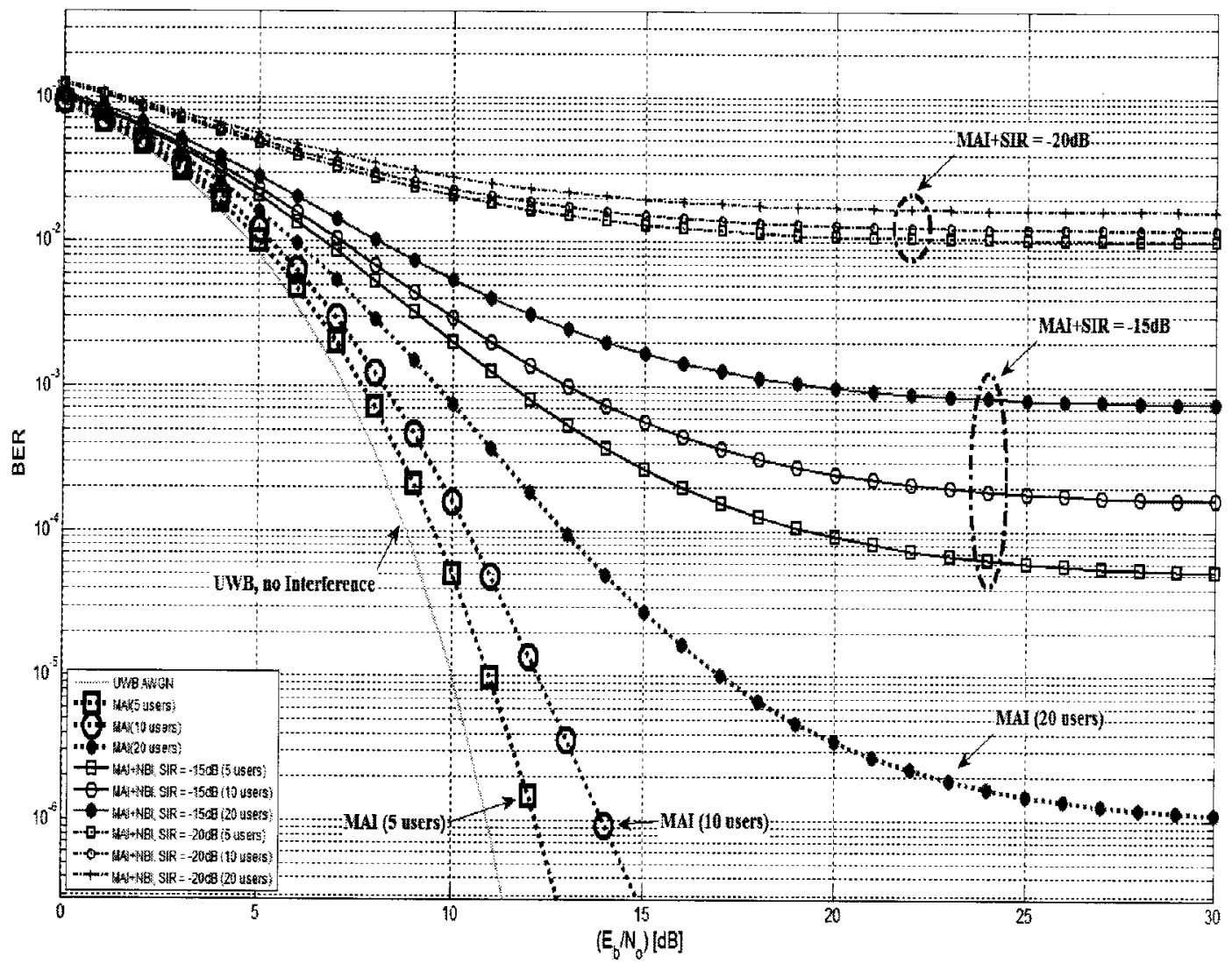

Figure 6.11: BER performance of a TH-PPM system in the presence of MAI and NBI signals

Figures 6.12 and 6.13 depict the BER performance of the ICS in the presence of the IEEE802.11a NBI signal with SIR $=-20 \mathrm{~dB}$, and MAI at different number of users (5, 10 and 20) for an av. Pse equals to 0.01 and 0.1 respectively. It can be seen that for $N_{u}=20$ user, the SNR degradation, with the use of the ICS, is reduced to be less than $3 \mathrm{~dB}$ and $9 \mathrm{~dB}$ respectively at $\mathrm{BER}=1 \times 10^{-4}$.

\subsubsection{Imperfect Power Control}

The impact of the presence of imperfect power control (IPC) MAI on the performance of the ICS will be investigated. The relation between the received energies of the interfered users to the received energy of the desired user ${ }^{6}$ in the following case study

${ }^{6}$ Assuming that the first user is the desired user and $N_{u}=20$ users. 


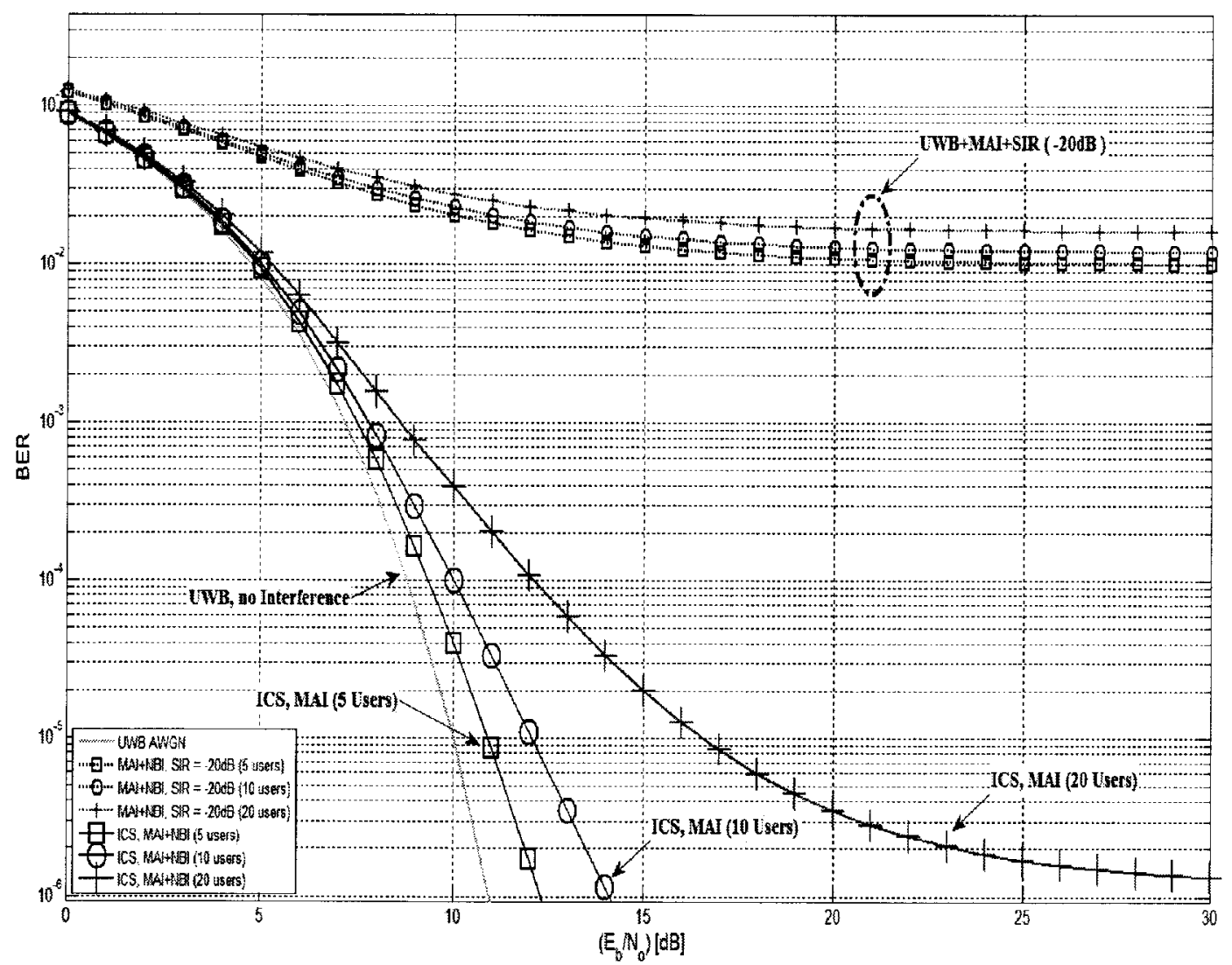

Figure 6.12: BER performance of the ICS in the presence of MAI and NBI with an av. Pse $=0.01$ 


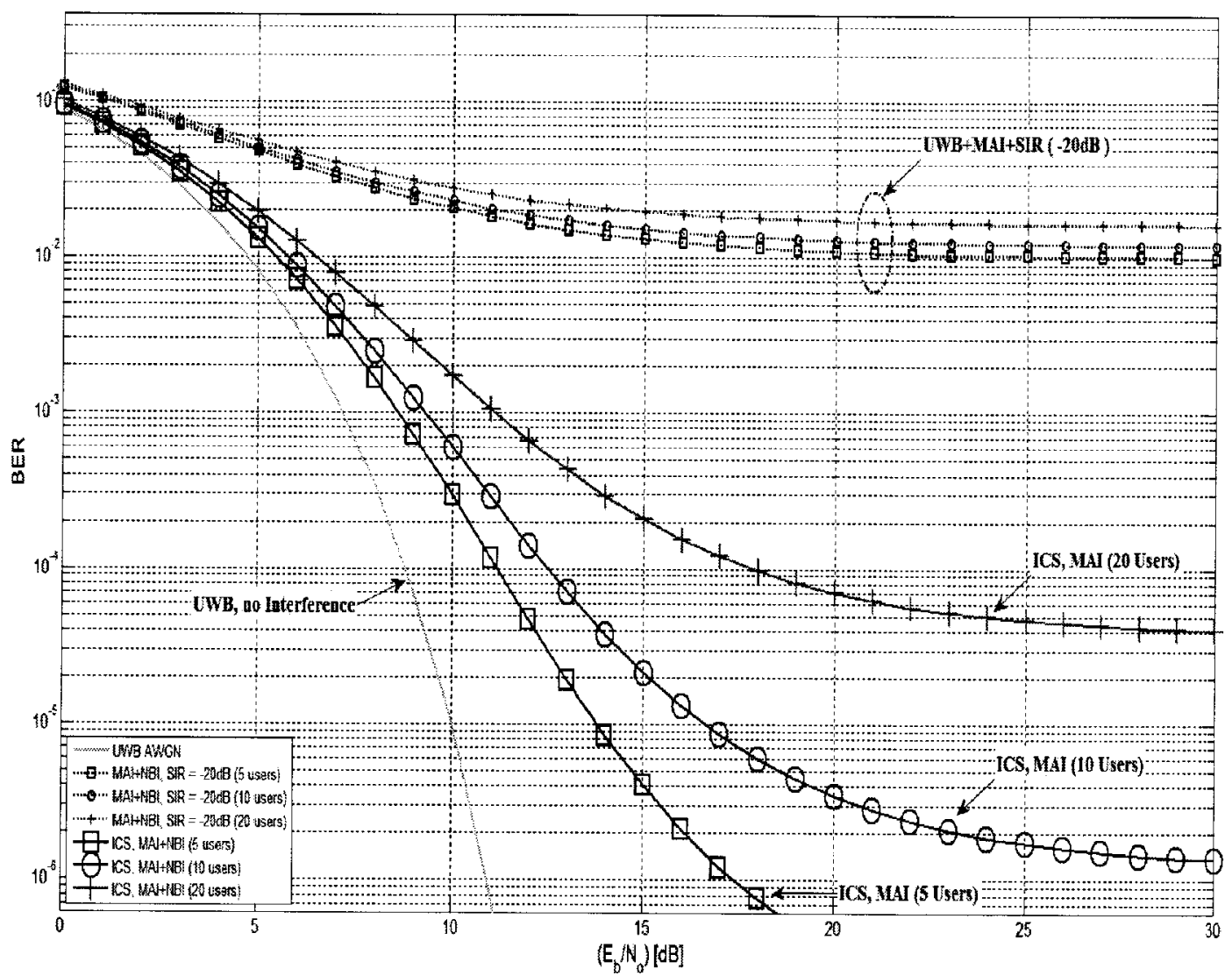

Figure 6.13: BER performance of the ICS in the presence of MAI and NBI with an av. Pse $=0.1$ 
can be written as [90]:

$$
\left\{\begin{array}{l}
\mathrm{E}_{R X}^{(2)}=E_{R X}^{(3)}=E_{R X}^{(4)}=E_{R X}^{(1)}, \\
\mathrm{E}_{R X}^{(5)}=E_{R X}^{(6)}=E_{R X}^{(7)}=E_{R X}^{(8)}=2 E_{R X}^{(1)} \\
\mathrm{E}_{R X}^{(9)}=E_{R X}^{(10)}=E_{R X}^{(11)}=E_{R X}^{(12)}=2 E_{R X}^{(1)} \\
\mathrm{E}_{R X}^{(13)}=E_{R X}^{(14)}=E_{R X}^{(15)}=E_{R X}^{(16)}=3 E_{R X}^{(1)} \\
\mathrm{E}_{R X}^{(17)}=E_{R X}^{(18)}=E_{R X}^{(19)}=E_{R X}^{(20)}=4 E_{R X}^{(1)}
\end{array}\right.
$$

Figure 6.14 depicts a comparison between the performance of a TH-PPM system in the presence of 20 users in PPC and IPC MAI. It can be seen that an additional SNR degradation, due to IPC MAI, is expected to be less than $6 \mathrm{~dB}$ at $\mathrm{BER}=2 \times 10^{-3}$.

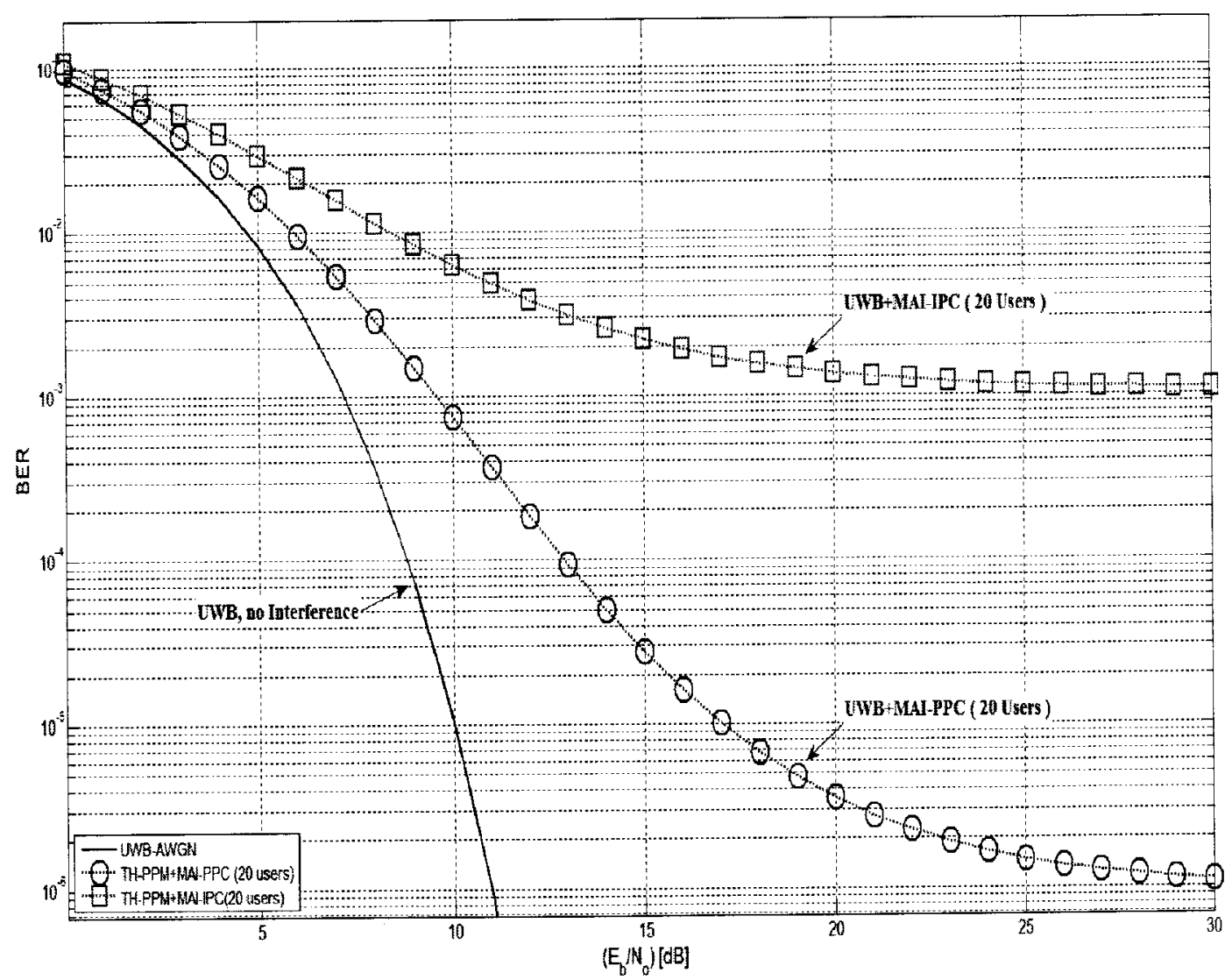

Figure 6.14: Comparison between the BER performances of a TH-PPM system in the presence of PPC and IPC MAI. 
The impact of NBI along with the presence of MAI on the performance of a TH-PPM system in both PPC and IPC MAI is depicted in figure 6.15 for SIR $=-10 \mathrm{~dB}$ and $-20 \mathrm{~dB}$.

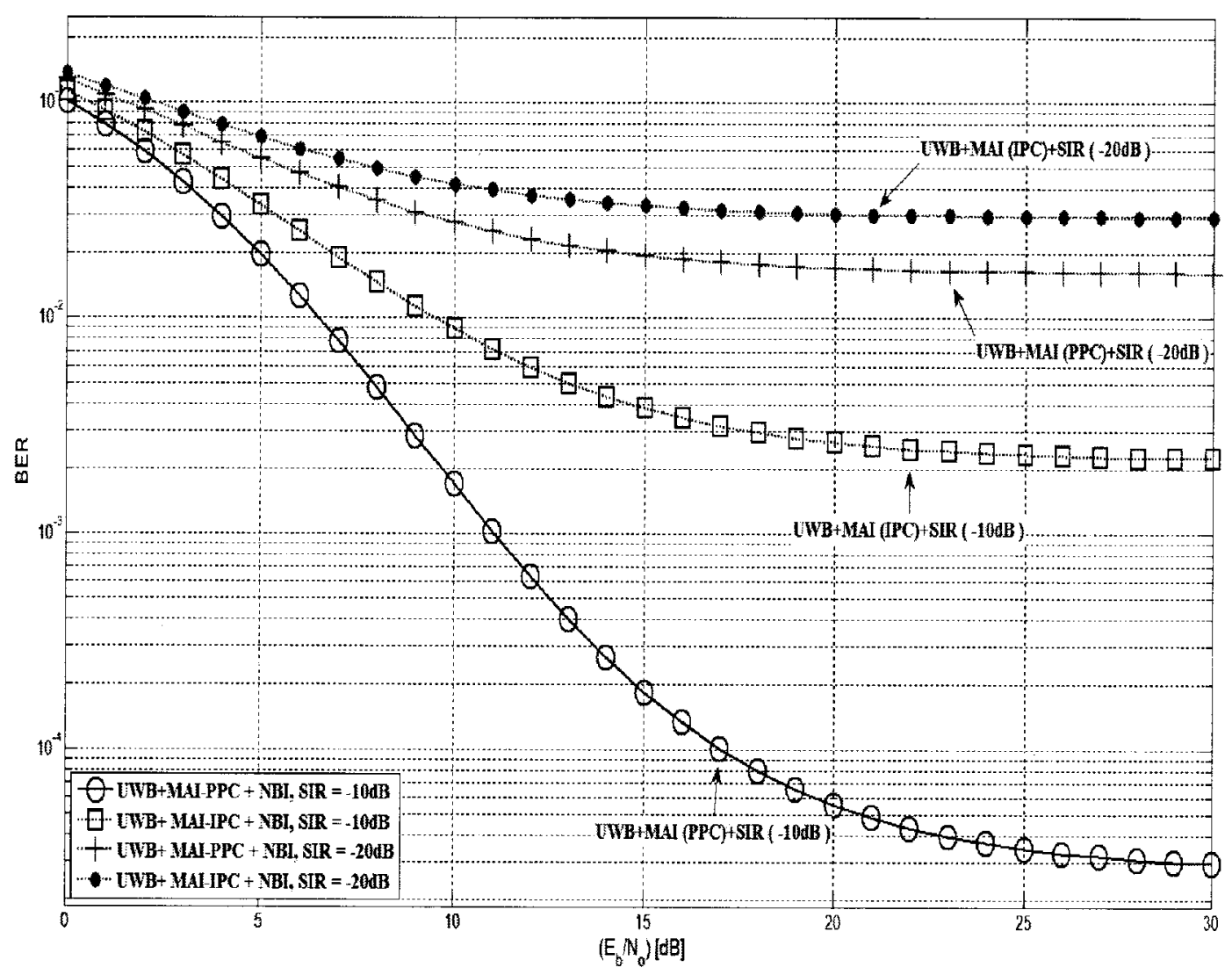

Figure 6.15: Comparison between the BER performances of a TH-PPM UWB system in the presence of NBI along with PPC and IPC MAI for 20 users.

The canceller efficiency in the presence of PPC and IPC MAI is depicted in figures 6.16 and 6.17 for $\mathrm{SIR}=-20 \mathrm{~dB}$ and the av. Pse is equal to 0.01 and 0.1 respectively. In figure 6.16 it can be seen that the SNR degradation is reduced to be less than 3 $\mathrm{dB}$ at $\mathrm{BER}=1 \times 10^{-4}$ in PPC MAI and less than $8 \mathrm{~dB}$ at BER $=1 \times 10^{-3}$ in IPC MAI. In figure 6.17 the SNR degradation is reduced to be less than $4 \mathrm{~dB}$ at $\mathrm{BER}=$ $1 \times 10^{-3}$ in PPC MAI and less than $5 \mathrm{~dB}$ at BER $=1 \times 10^{-2}$ in IPC MAI. 


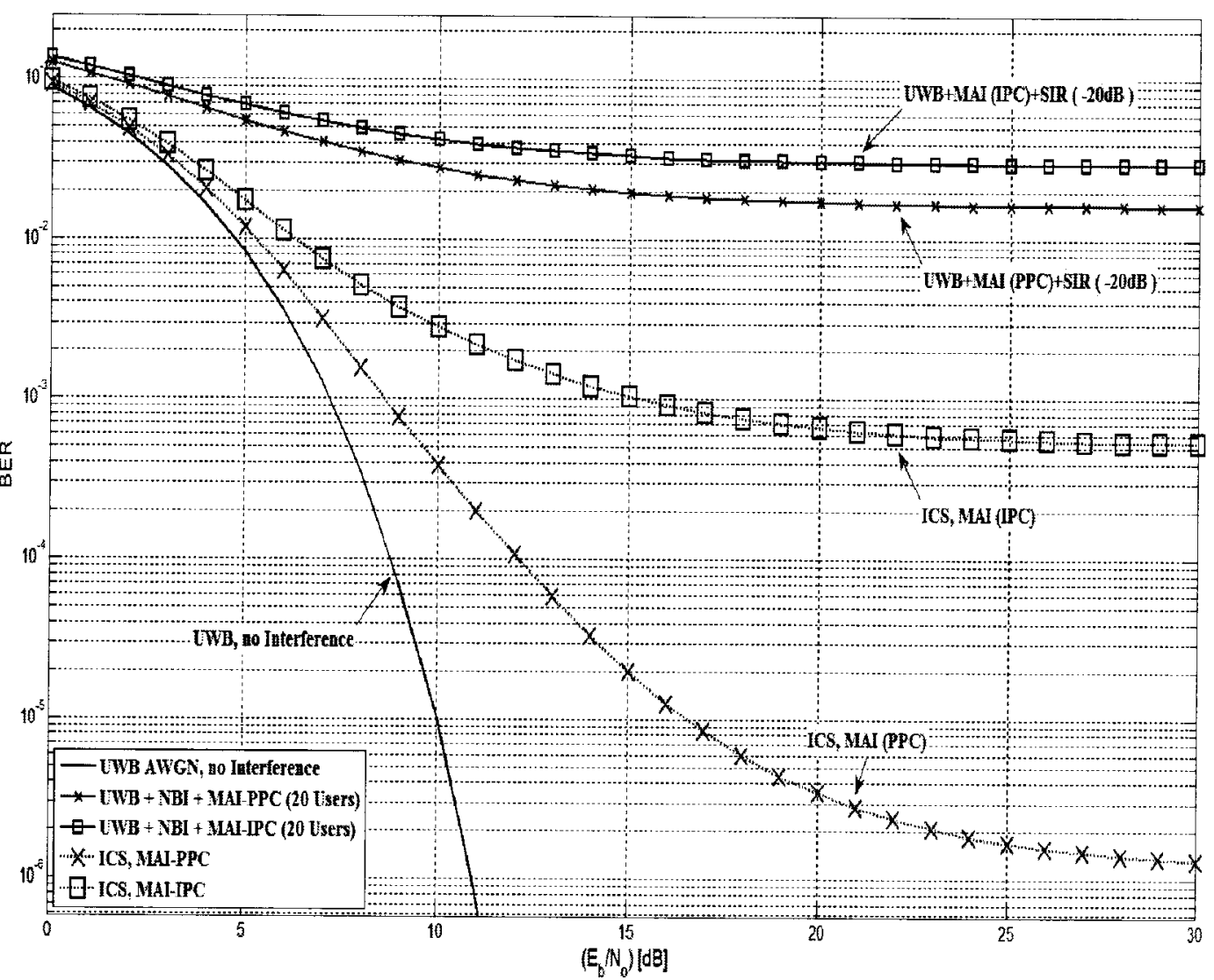

Figure 6.16: Comparison between the ICS BER performance in the presence of NBI, PPC and IPC MAI for 20 users, SIR $=-20 \mathrm{~dB}$ and with av. Pse $=0.01$. 


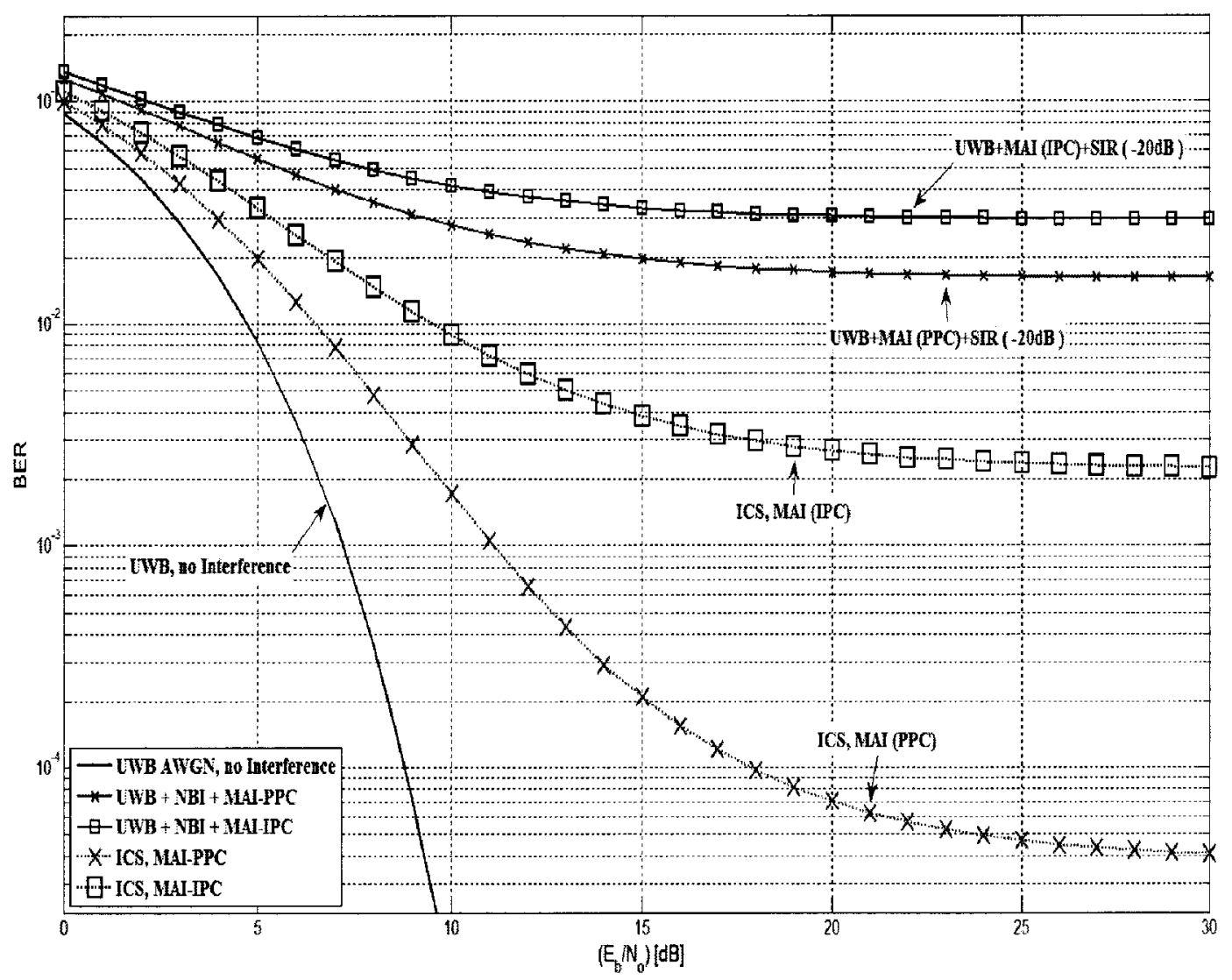

Figure 6.17: Comparison between the ICS BER performance in the presence of NBI, PPC and IPC MAI for 20 users, SIR $=-20 \mathrm{~dB}$ and with av. Pse $=0.1$. 


\subsubsection{Nakagami-m and Log-normal Flat Fading Channels}

\subsubsection{Perfect Power Control}

In this section, the BER performance of an IR-UWB system using the ICS in the presence of MAI in both Nakagami-m and Log-normal flat fading channels is presented.

The BER performance of a TH-PPM system in the presence of NBI and MAI impacts in a Nakagami-m flat fading channel can be evaluated using equation (3.17), where the variable $\eta$ is represented in equation (6.9).

Figure 6.18 depicts the performance of a TH-PPM system in the presence of both MAI and NBI with $R_{b}=20 \mathrm{Mbps}, \mathrm{m}=2$, SIR $=-10 \mathrm{~dB}$ and $-20 \mathrm{~dB}$. It can be seen that at $\mathrm{SIR}=-10 \mathrm{~dB}$, the SNR degradation is expected to be less than $2 \mathrm{~dB}$, $3 \mathrm{~dB}$ and $8 \mathrm{~dB}$ for number of users $N_{u}=5,10$ and 20 users respectively at BER $=$ $1 \times 10^{-2}$.

Figures 6.19 and 6.20 depict the BER performance of the ICS in the presence of MAI and SIR $=-20 \mathrm{~dB}$ at an av. Pse equals to 0.01 and 0.1 respectively.

It can be seen that, the SNR degradation is reduced to be $1 \mathrm{~dB}$ and $2 \mathrm{~dB}$ for an av. Pse equals to 0.01 and 0.1 respectively at $\mathrm{BER}=1 \times 10^{-2}$ and for $N_{u}=5$ users.

For a Log-normal flat fading channel, figure 6.21 depicts the performance of the TH-PPM system in the presence of both MAI and NBI with SIR $=-10$ and -20 $\mathrm{dB}$ and dB-spread equals to $2 \mathrm{~dB}$. It can be seen that for SIR $=-10 \mathrm{~dB}$, the SNR degradation is expected to reach up to $2 \mathrm{~dB}, 3 \mathrm{~dB}$ and $10 \mathrm{~dB}$ for $N_{u}=5,10$ and 20 users respectively at $\mathrm{BER} \approx 6 \times 10^{-3}$.

The performance of the ICS is depicted in figures 6.22 and 6.23 for an av. Pse equals to 0.01 and 0.1 respectively and SIR $=-20 \mathrm{~dB}$. It can be seen that for an av. Pse $=$ 0.01 , the SNR degradation is expected to be less than $2 \mathrm{~dB}$ and $4 \mathrm{~dB}$ at $N_{u}=5$ and 10 users respectively and at $\mathrm{BER}=1 \times 10^{-3}$.

\subsubsection{Imperfect Power Control}

A comparison between the performances of the TH-PPM in PPC and IPC MAI is depicted in figure 6.24 for $\mathrm{SIR}=-10 \mathrm{~dB}$ and $-20 \mathrm{~dB}, N_{u}=20$ users and a Nakagami fading parameter $m=2$. It can see that, an additional SNR degradation, due to IPC MAI, is expected to be less than $6 \mathrm{~dB}$ at $\mathrm{BER}=3 \times 10^{-2}$. 


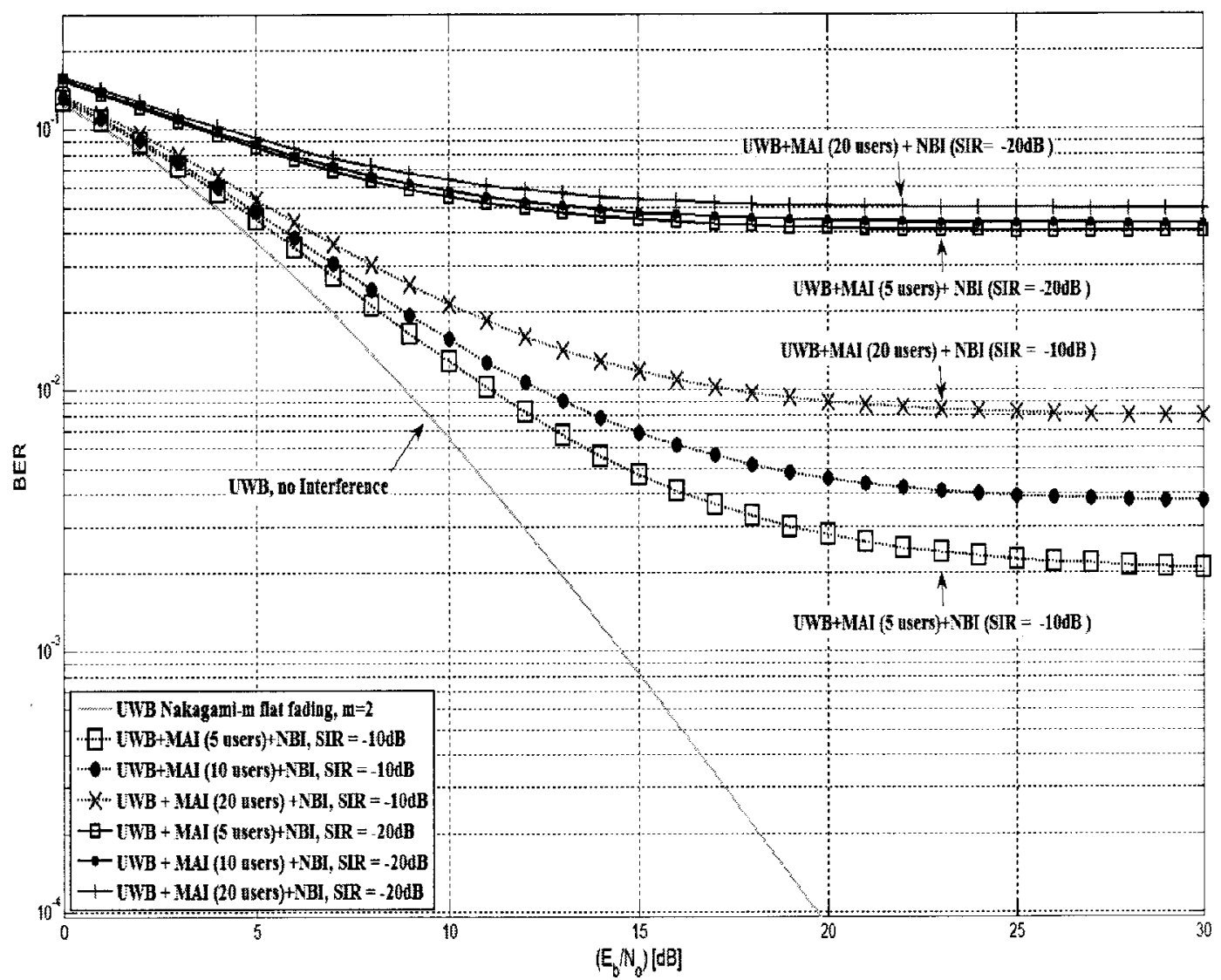

Figure 6.18: BER performance of a TH-PPM UWB system in the presence of PPC MAI and NBI in a Nakagami-m flat fading channel. 


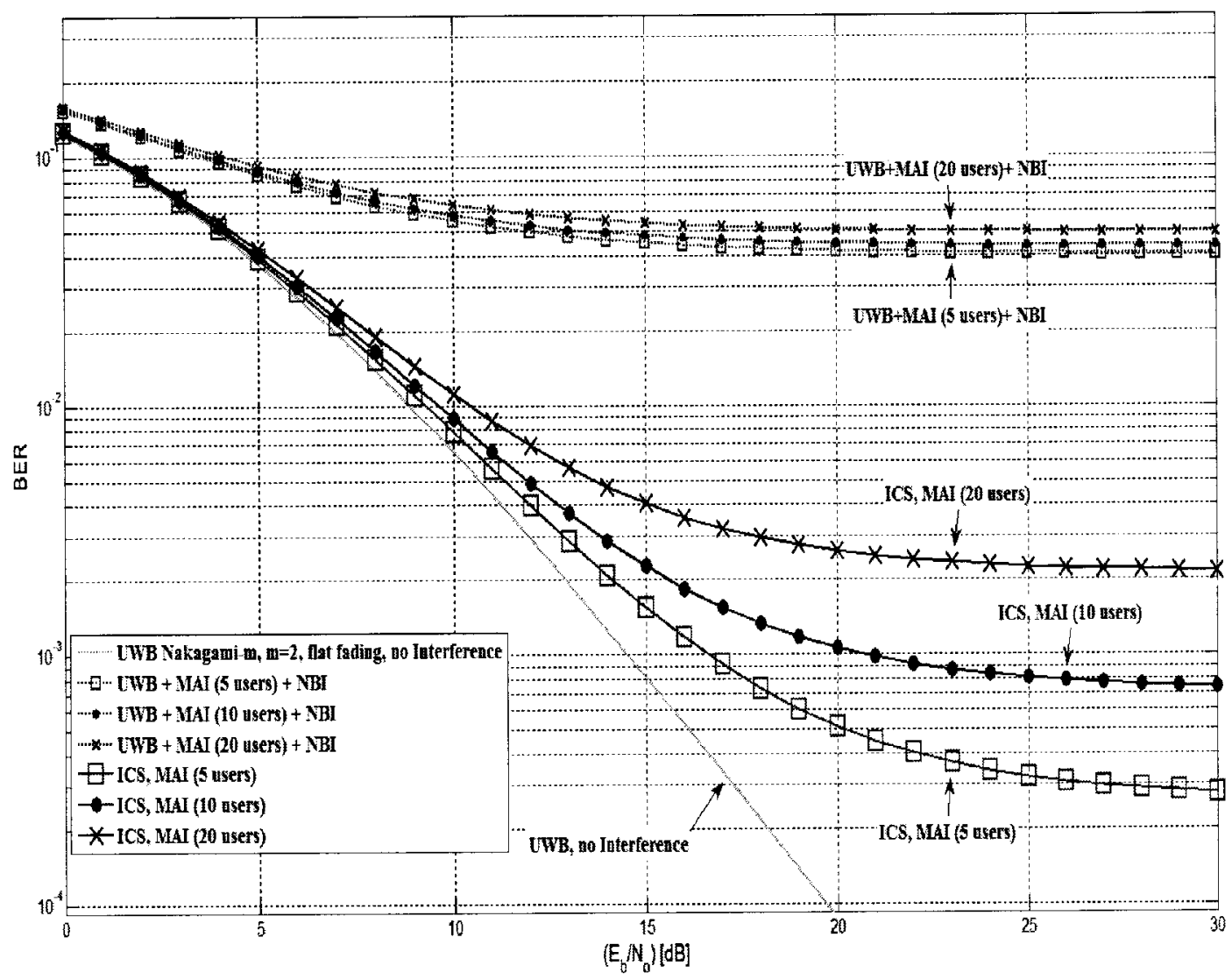

Figure 6.19: ICS BER performance in the presence of PPC MAI and NBI in a Nakagami-m flat fading channel, $\mathrm{m}=2, \mathrm{SIR}=-20 \mathrm{~dB}$ and av. $P_{s e}=0.01$. 


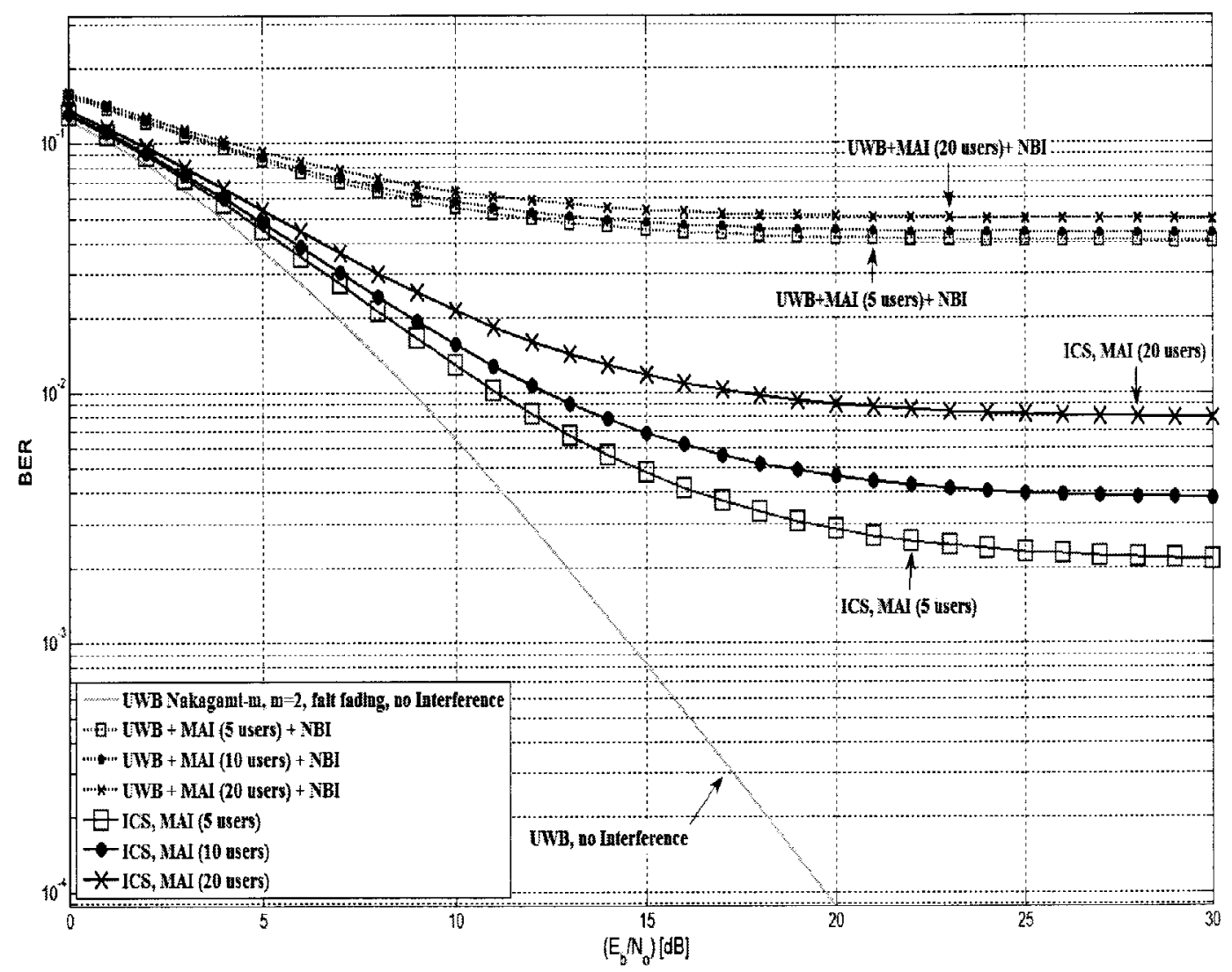

Figure 6.20: ICS BER performance in the presence of PPC MAI and NBI in a Nakagami-m flat fading channel, $\mathrm{m}=2, \mathrm{SIR}=-20 \mathrm{~dB}$ and av. $P_{s e}=0.1$. 


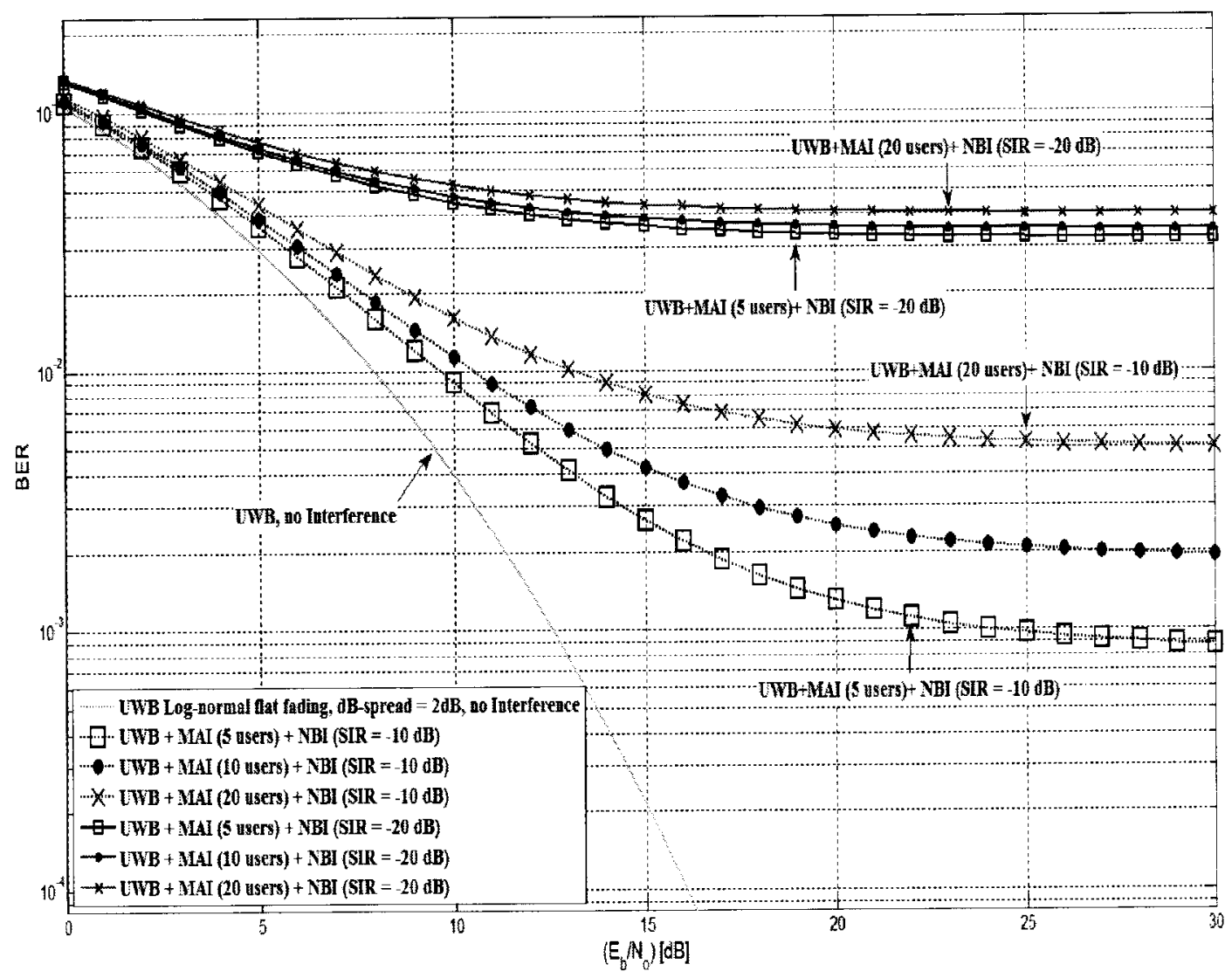

Figure 6.21: BER performance of a TH-PPM system in the presence of PPC MAI and NBI in a Log-normal flat fading channcl. 


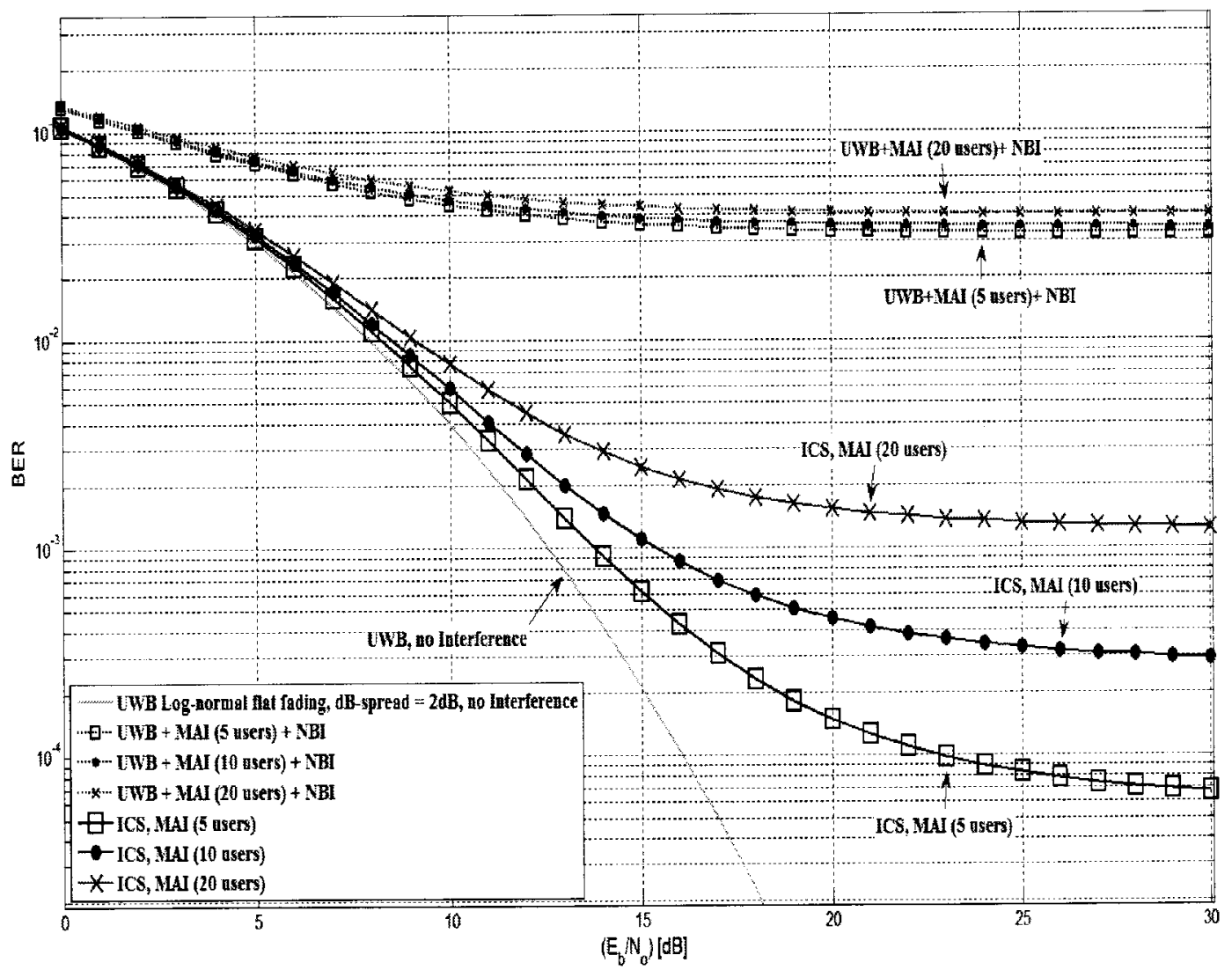

Figure 6.22: ICS BER performance in the presence of PPC MAI and NBI in a Log-normal flat fading channel, av. $P_{s e}=0.01, \mathrm{~dB}$-spread $=2 \mathrm{~dB}$ and $\mathrm{SIR}=$ $-20 \mathrm{~dB}$. 


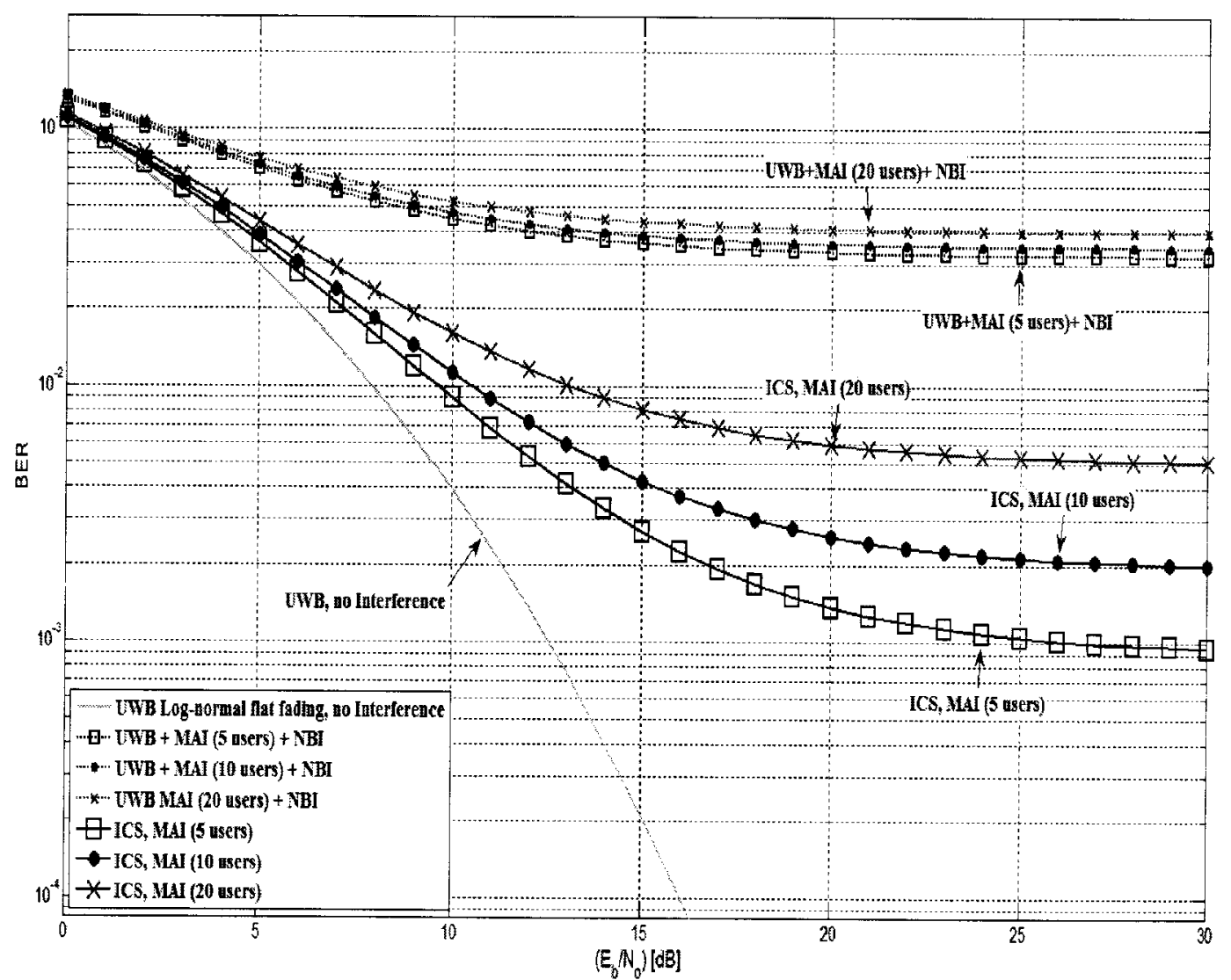

Figure 6.23: ICS BER performance in the presence of PPC MAI and NBI in a Log-normal flat fading channel, av. $P_{s e}=0.1, \mathrm{~dB}$-spread $=2 \mathrm{~dB}$ and $\mathrm{SIR}=$ $-20 \mathrm{~dB}$. 


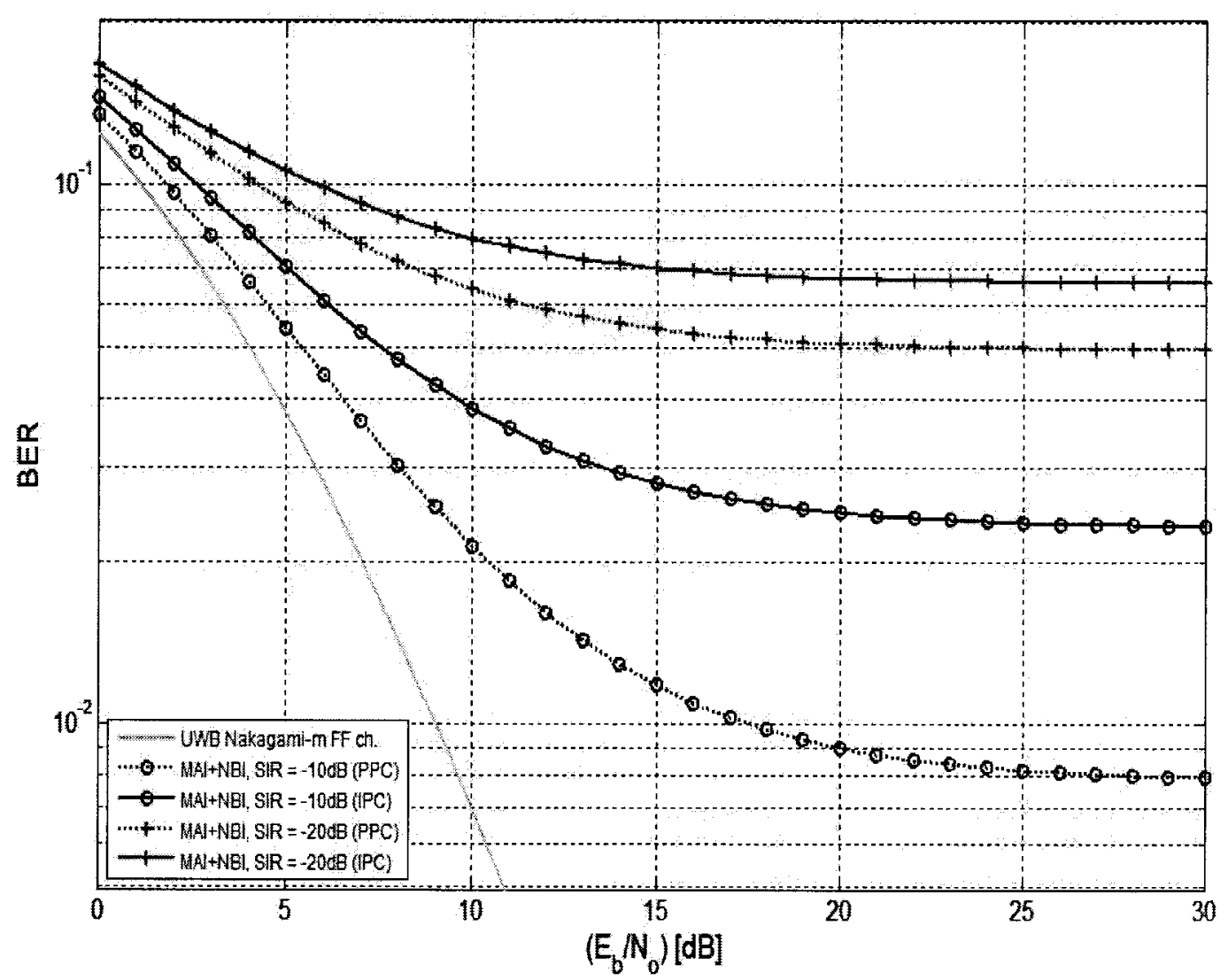

Figure 6.24: BER performance comparison of a TH-PPM system in PPC and IPC MAI in the presence of NBI in a Nakagami-m flat fading channel, $N_{u}=20$ users and $\mathrm{m}=2$. 
Figures 6.25 and 6.26 depict the ICS BER performance in PPC and IPC MAI for an av. Pse equals to 0.01 and 0.1 respectively.

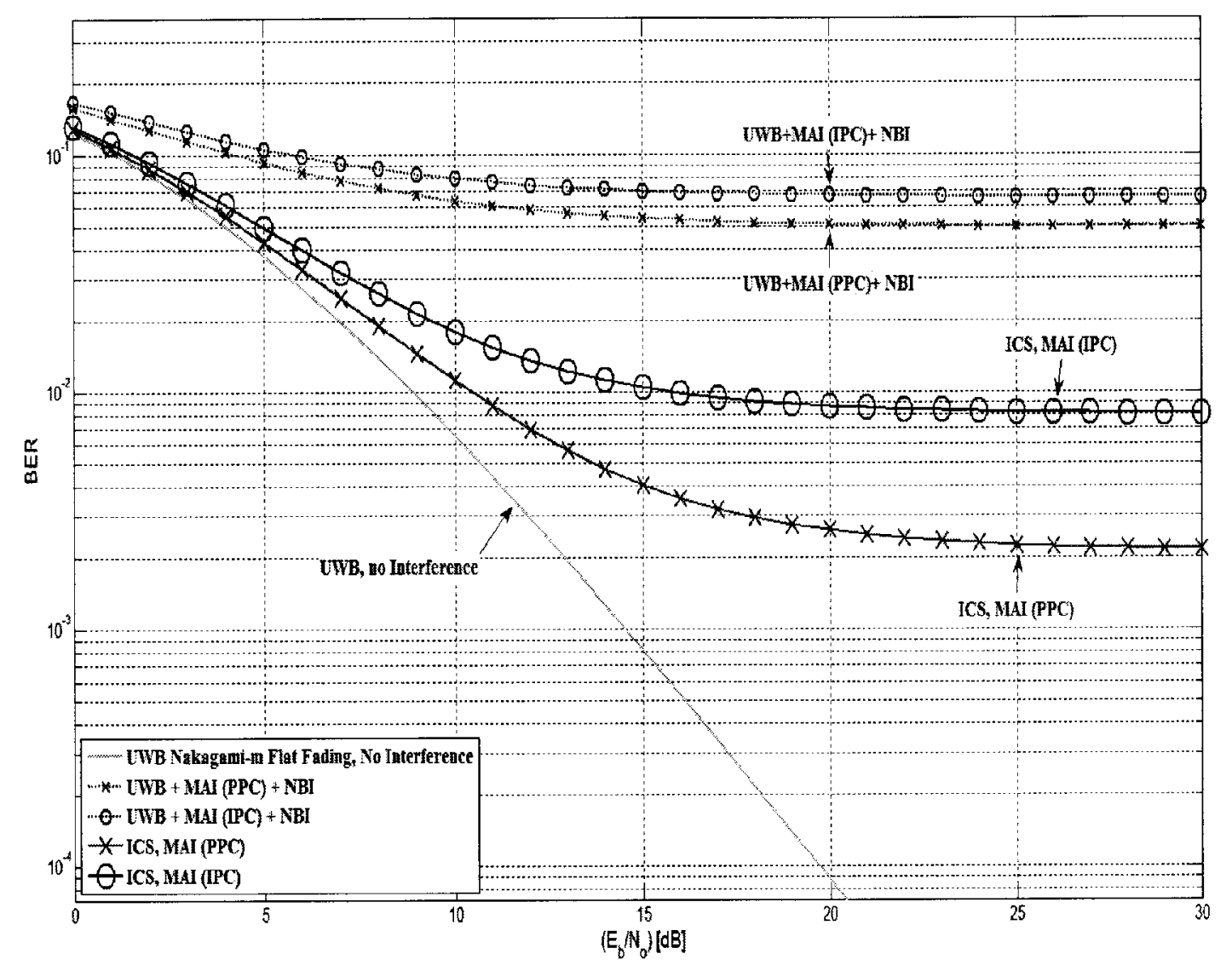

Figure 6.25: ICS BER performance in PPC and IPC MAI in a Nakagami-m flat fading channel, av. Pse $=0.01, N_{u}=20$ users and $\mathrm{m}=2$.

It can be seen that the additional SNR degradation, due to IPC MAI, is expected to be be less than $5 \mathrm{~dB}$ at $\mathrm{BER}=1 \times 10^{-2}$ and less than $4 \mathrm{~dB}$ at $\mathrm{BER}=3 \times 10^{-2}$ for an av. Pse $=0.01$ and 0.1 respectively.

In a Log-normal flat fading channel the performance of the ICS is depicted in figures 6.27 and 6.28 for $\mathrm{dB}$ spread $=2 \mathrm{~dB}$ and av. Pse $=0.01$ and 0.1 respectively. It can be seen that the additional SNR degradation is expected to be less than $3 \mathrm{~dB}$ at $\mathrm{BER}=1 \times 10^{-2}$ and less than $6 \mathrm{~dB}$ at $\mathrm{BER}=2 \times 10^{-2}$ for an av. Pse $=0.01$ and 0.1 respectively. 


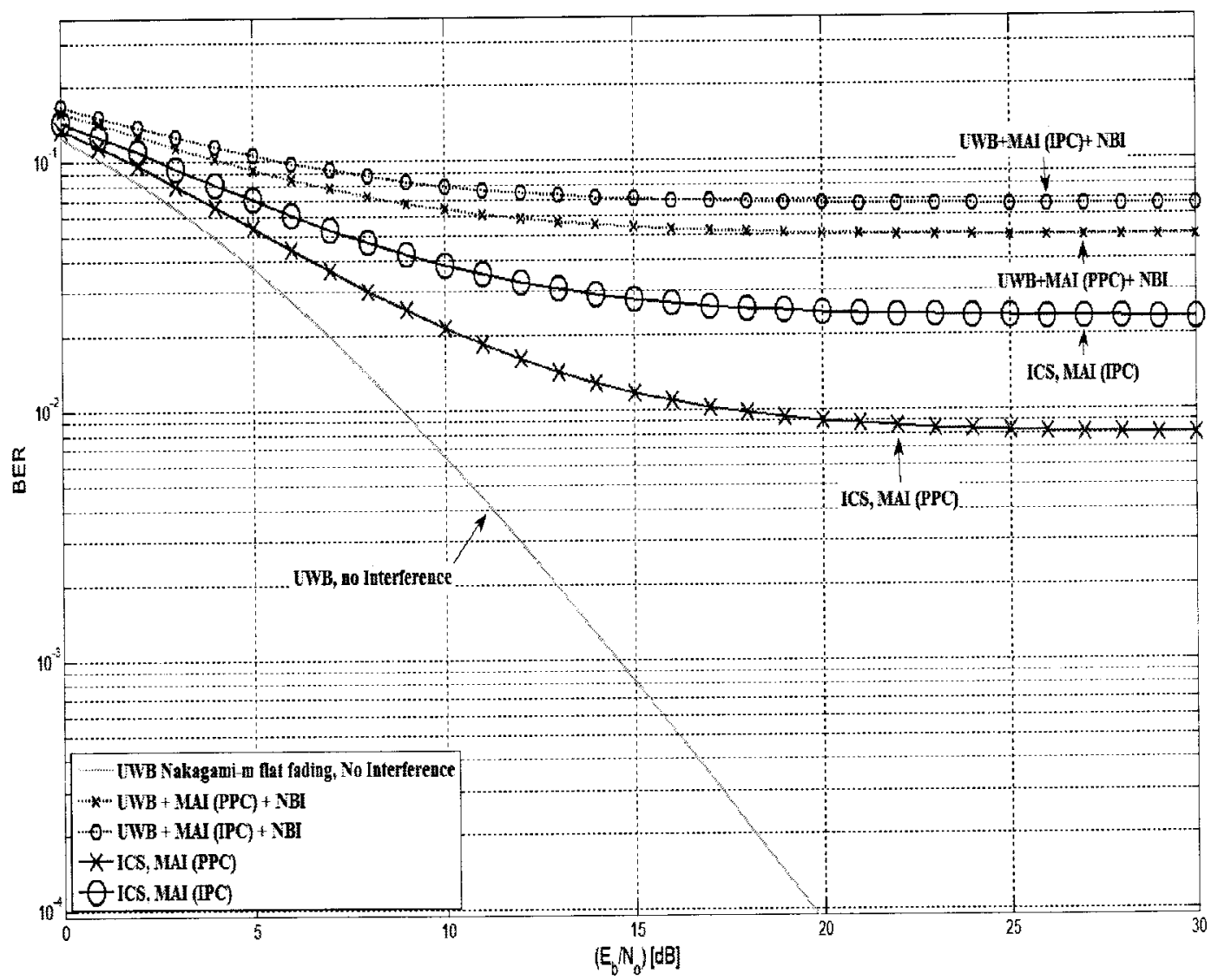

Figure 6.26: ICS BER performance in PPC and IPC MAI in a Nakagami-m flat fading channel, av. Pse $=0.1, N_{u}=20$ users and $\mathrm{m}=2$. 


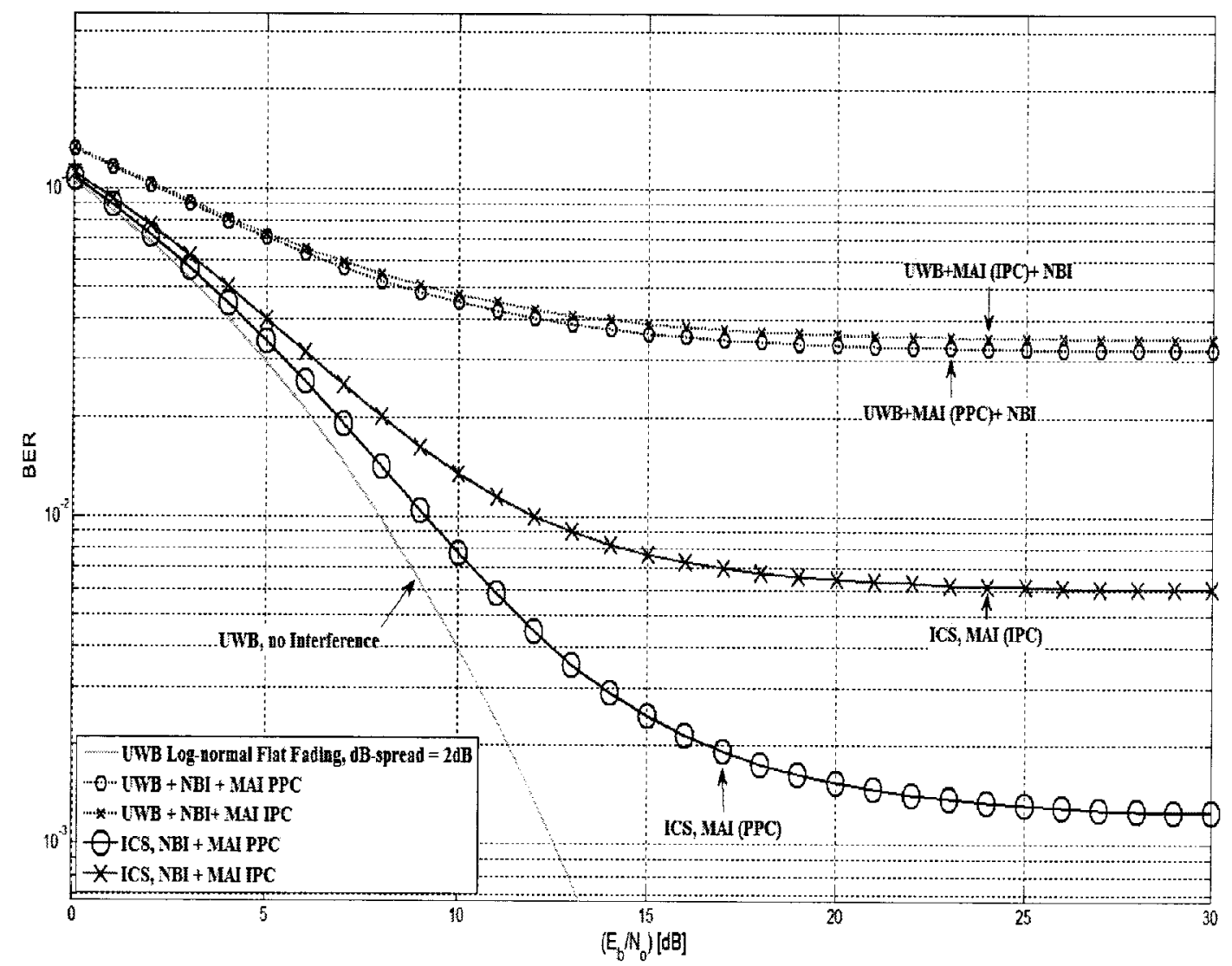

Figure 6.27: ICS BER performance in the presence of PPC and IPC MAI in a Log-normal flat fading channel, av. $P_{s e}=0.01$ and $N_{u}=20$ users. 


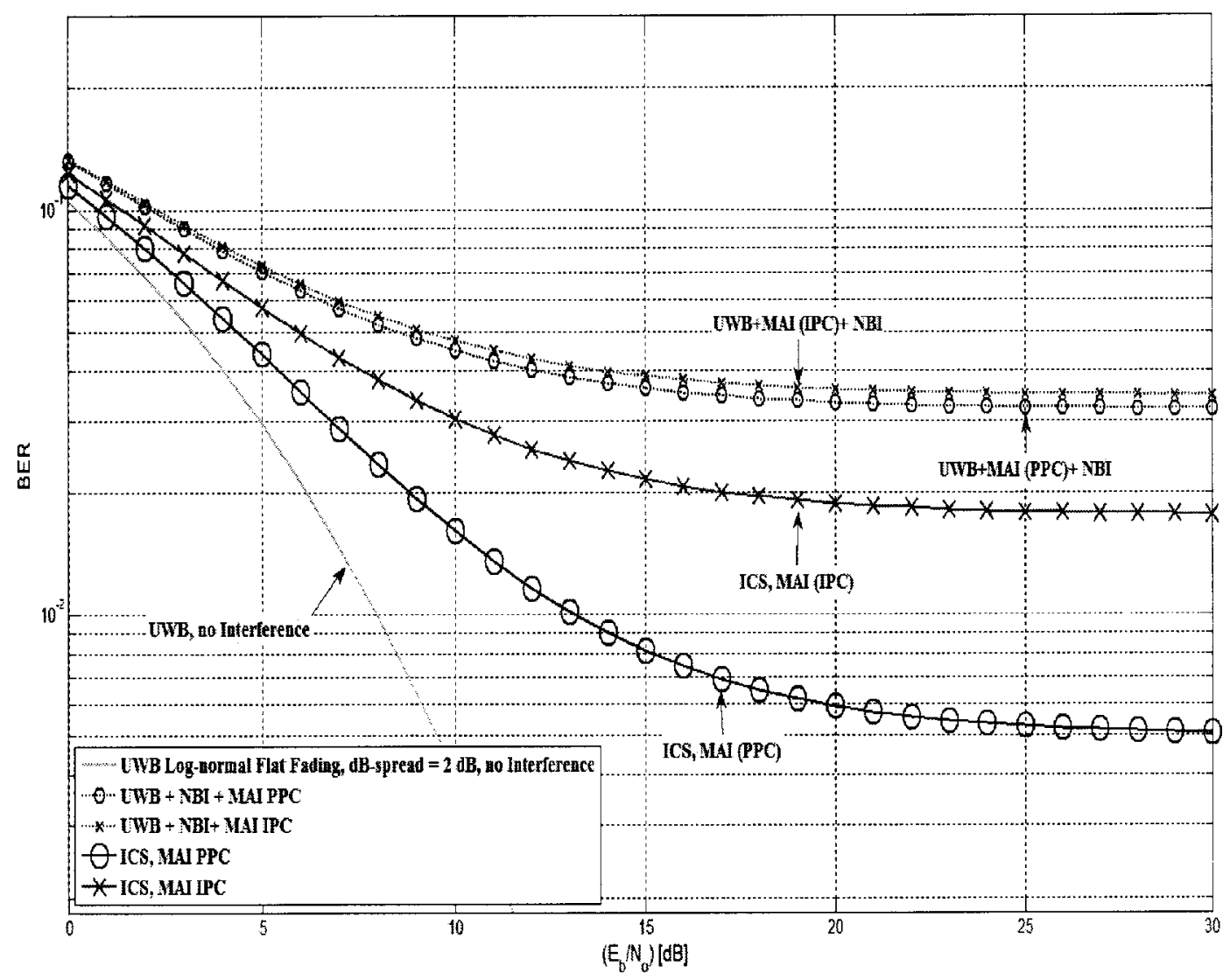

Figure 6.28: ICS BER performance in the presence of PPC and IPC MAI in a Log-normal flat fading channel, av. $P_{s e}=0.1$ and $N_{u}=20$ users. 


\subsubsection{In the IEEE802.15.3a UWB Channel Models}

The impact of the presence of MAI on the ICS is investigated under the following assumptions: the number of clusters $\mathrm{L}=2$ and the number of rays within each cluster $\mathrm{K}=20$. The UWB data rate is $100 \mathrm{Mbps}, \tau_{p}=0.192 \mathrm{~ns}$ and the NBI is modeled as the standard IEEE802.11a signal operating at center frequency $f_{i}=5.745 \mathrm{GHz}$.

\subsubsection{Perfect Power Control}

The performance of a UWB system in the presence of NBI in the IEEE802.15.3a channel models was presented in chapter $(4)^{7}$. In order to add the impact of MAI, the conditional BER conditioned on $(\mathbf{a} ; \mathbf{t})$ will be modified as

$$
P_{e \mid \mathbf{a}, \mathbf{t}}=Q(\sqrt{\gamma(\mathbf{a}, \mathbf{t})})
$$

where

$$
\begin{gathered}
\gamma(\mathbf{a}, \mathbf{t})=\frac{N_{c} E_{g} a_{s}^{2}}{\sigma_{n}^{2}+\sigma_{i}^{2}+\sigma_{M A I}^{2}} \\
\sigma_{M A I}^{2}=\left(\frac{(1-\varrho)^{2} \cdot \gamma_{R}}{\sigma_{M}^{2} \cdot R_{b} \cdot \sum_{n=2}^{N_{u}} \frac{E_{R X}^{(n)}}{E_{R X}^{(1)}}}\right)^{-1}
\end{gathered}
$$

Figures 6.29 and 6.30 depict the BER performance of a DS-BPSK system in the presence of both NBI and PPC MAI for different number of users $\left(N_{u}=5,10\right.$ and 20) and $\mathrm{SIR}=-15 \mathrm{~dB}$ in the CM1 and the CM4 channel models respectively. It can be seen that and for $N_{u}=5$ users, an additional SNR degradation is expected to reach up to $4 \mathrm{~dB}$ and $8 \mathrm{~dB}$, due to $\mathrm{NBI}$, in the CM1 and the CM4 channel models respectively.

Figures 6.31 and 6.32 depict the ICS BER performance in the CM1 and the CM4 channel models respectively for av. Pse equals to 0.05 and $0.1, \mathrm{SIR}=-20 \mathrm{~dB}$ and for different number users $\left(N_{u}=10\right.$ and 20 users).

It can be seen that, in the CM1 channel model for an av. Pse $=0.05$ and for $N_{u}=10$ and 20 users respectively, the additional SNR degradation is expected to reach up to $1.56 \mathrm{~dB}$ and $4.13 \mathrm{~dB}$ at $\mathrm{BER}=1 \times 10^{-3}$. Whereas, for an av. Pse =

\footnotetext{
7 As presented in equations (5.82) - (5.85).
} 


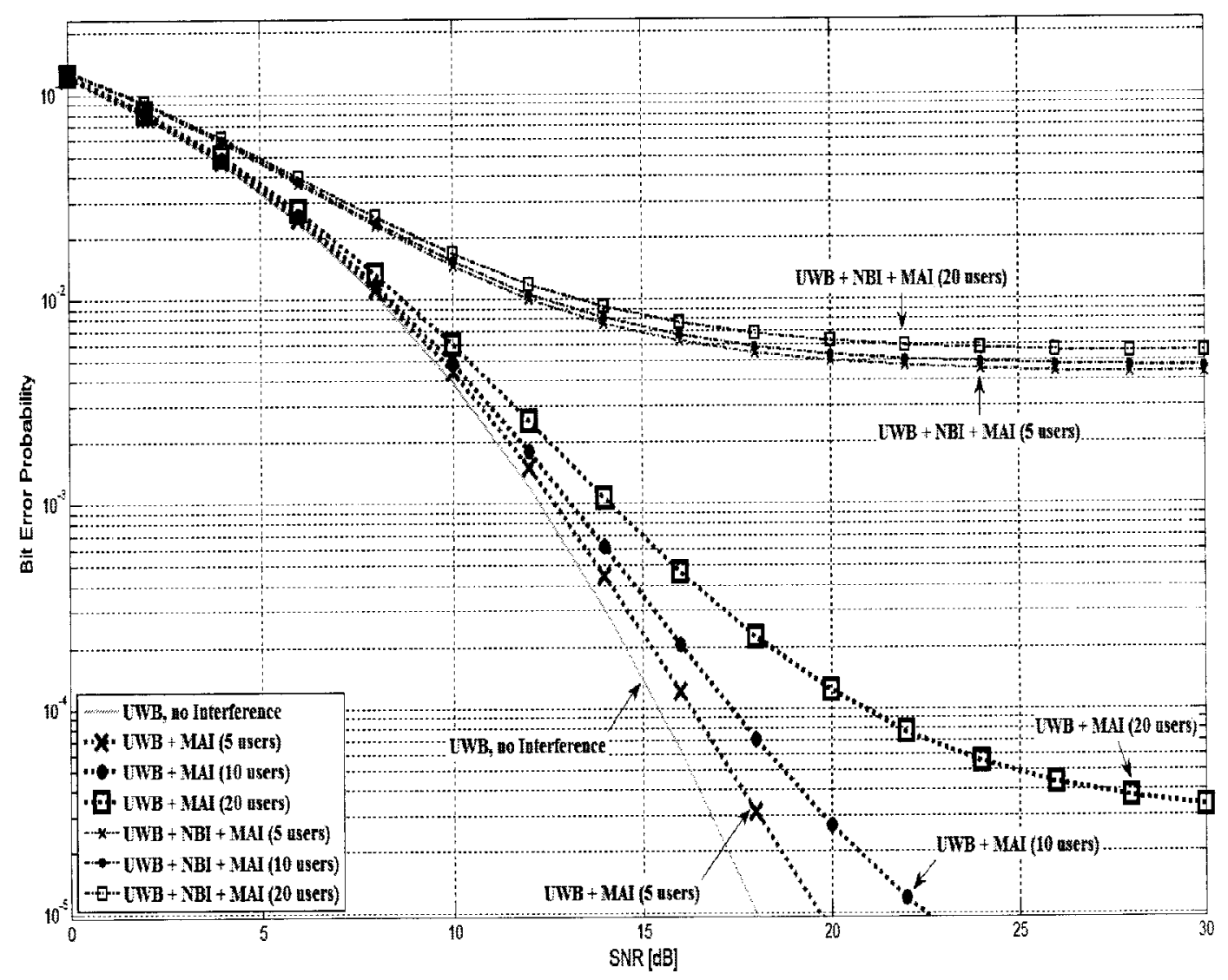

Figure 6.29: BER performance of the DS-BPSK system in the presence of NBI and PPC MAI in the CM1 channel model. 


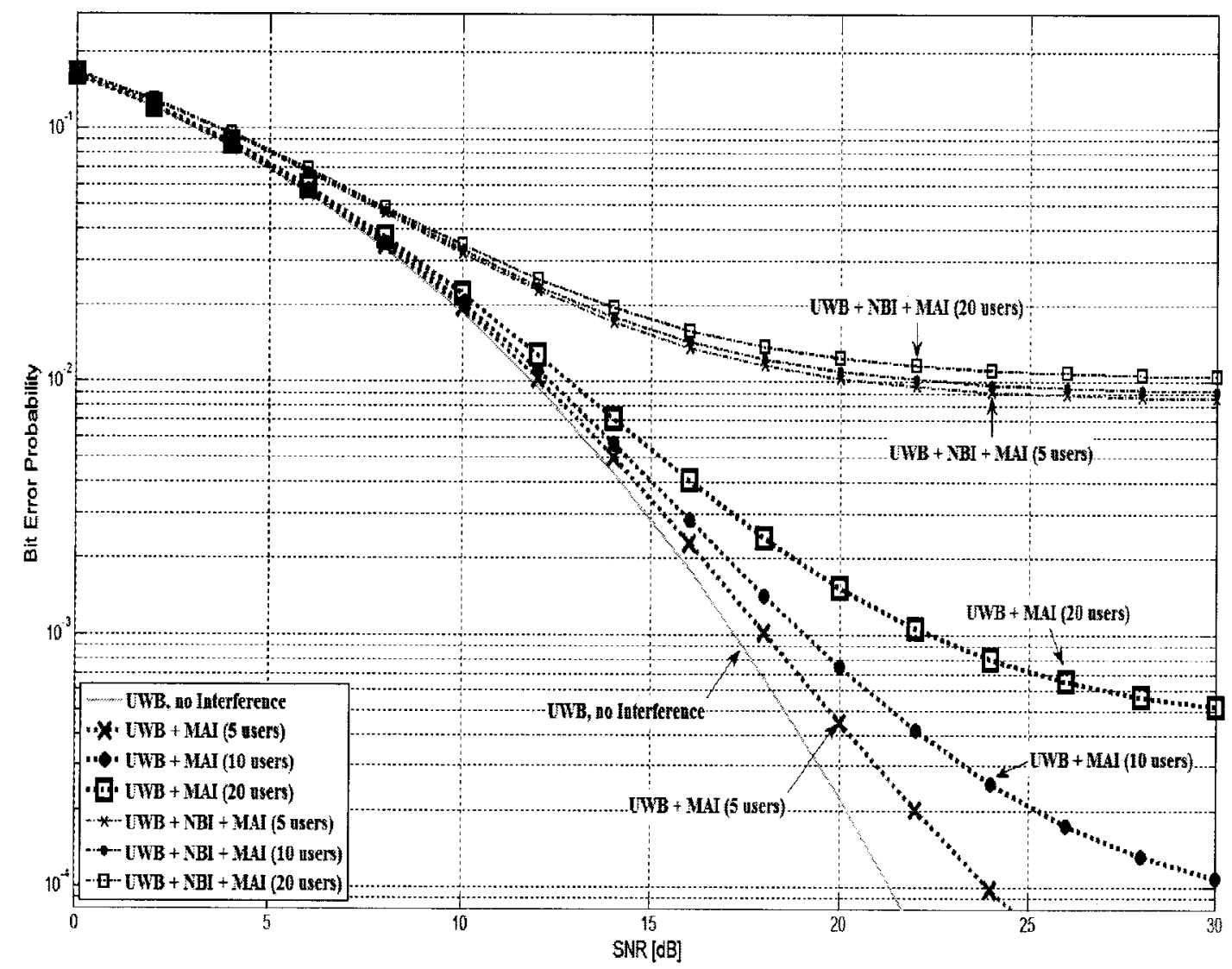

Figure 6.30: BER performance of the DS-BPSK-UWB in the presence of NBI and PPC MAI in the CM4 channel model. 


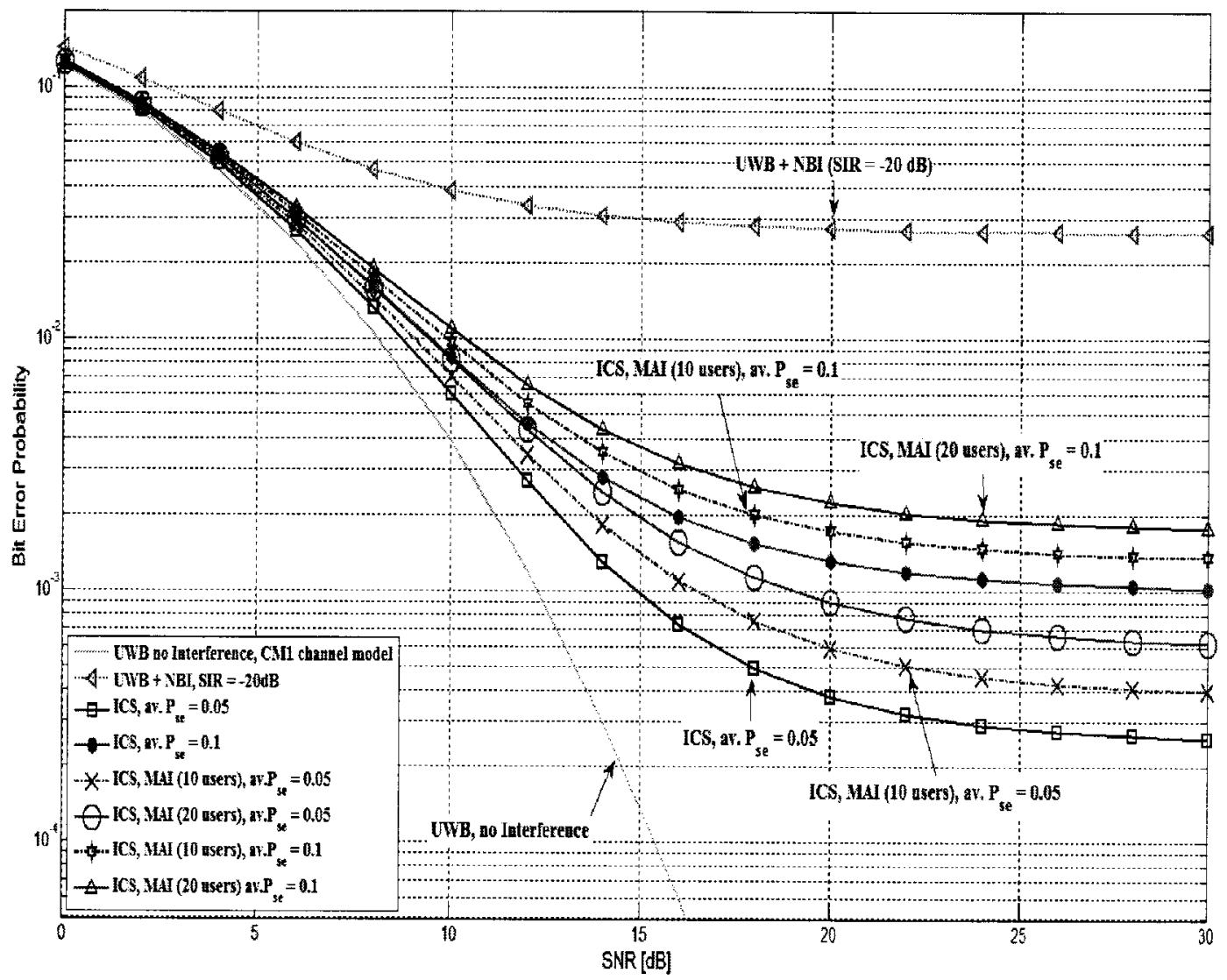

Figure 6.31: BER performance of the ICS in the presence of NBI and PPC MAI in the $\mathrm{CM} 1$ channel model and $\mathrm{SIR}=-20 \mathrm{~dB}$.

0.1 , the additional SNR degradation is expected to reach up to $2.13 \mathrm{~dB}$ and $6.69 \mathrm{~dB}$ respectively at $\mathrm{BER}=2 \times 10^{-2}$.

In the CM4 channel model, for $N_{u}=10$ and 20 users respectively, the additional SNR degradation at an av. Pse $=0.05$ is expected to reach up to $1.79 \mathrm{~dB}$ and 4.75 $\mathrm{dB}$ at $\mathrm{BER}=3 \times 10^{-2}$ and at an av. Pse $=0.1$ is expected to reach up to $2.46 \mathrm{~dB}$ and $8 \mathrm{~dB}$ at $\mathrm{BER}=5 \times 10^{-2}$.

\subsubsection{Imperfect Power Control}

The impact of IPC MAI on the performance of the ICS is depicted in figures (6.33) and (6.34) in the CM1 and the CM4 channel models respectively for an av. Pse = $0.05, \mathrm{SIR}=-20 \mathrm{~dB}$ and for $N_{u}=20$ users. It can be seen that in the CM1 model, an additional SNR degradation is expected to reach up to $3.64 \mathrm{~dB}$ at $\mathrm{BER}=3 \times 10^{-3}$, 


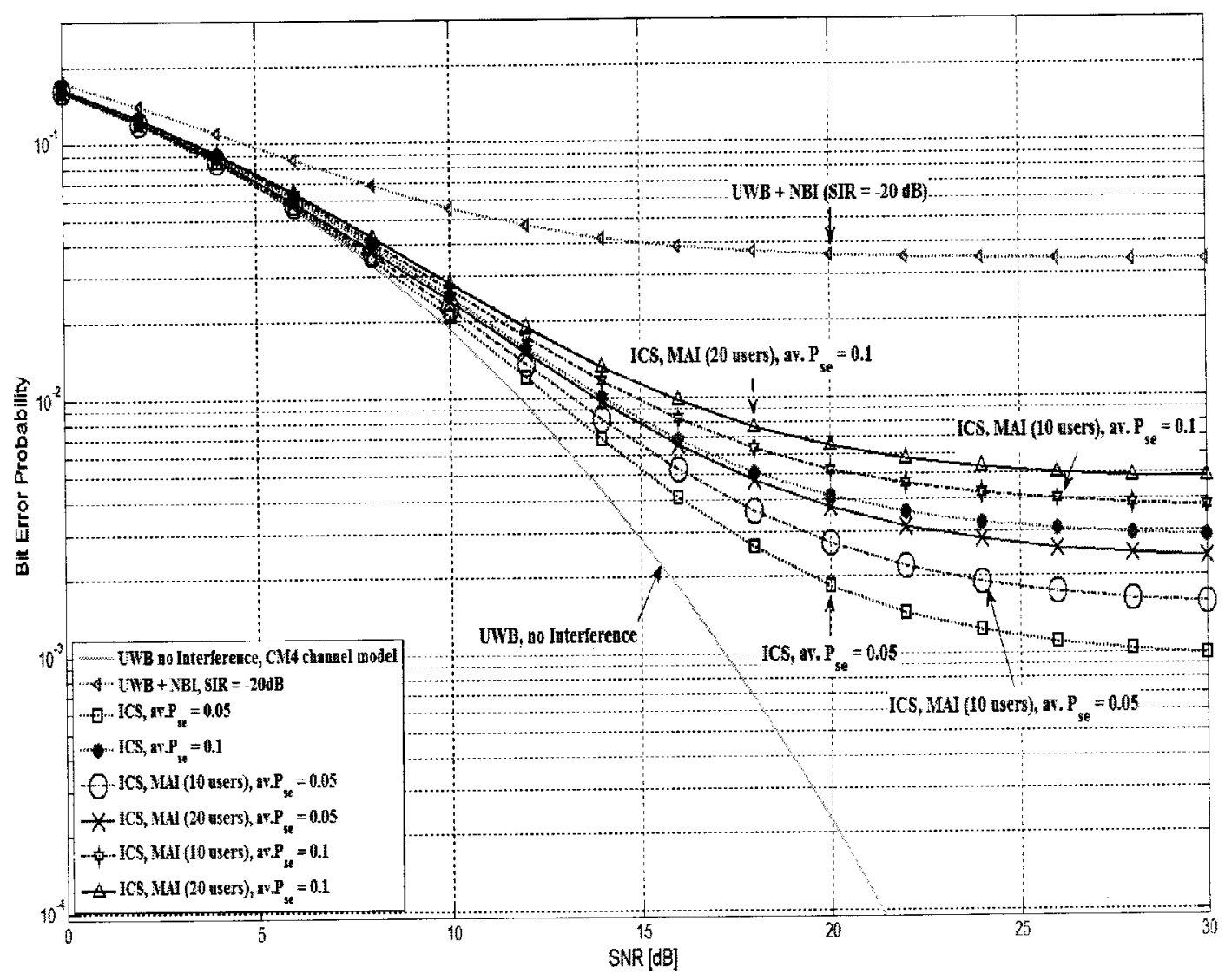

Figure 6.32: BER performance of the ICS in the presence of NBI and PPC MAI in the CM4 channel model and SIR $=-20 \mathrm{~dB}$.

whereas in the CM4 channel model, the additional SNR degradation is expected to reach up to $5 \mathrm{~dB}$ at $\mathrm{BER}=7 \times 10^{-3}$.

\subsection{Performance Comparison with a Notch Filter Based Case}

The performance effectiveness of the ICS is analyzed by making a comparison with the performance obtained by using a notch filter. This comparison is done under the following assumptions: the UWB channel is modeled as the IEEE802.15.3a CM1 and CM4 channel models, the number of clusters $\mathrm{L}=2$ and the number of rays within each cluster $\mathrm{K}=20$. The UWB data rate is $100 \mathrm{Mbps}$ and $\mathrm{SIR}=-15 \mathrm{~dB}$. The notch 


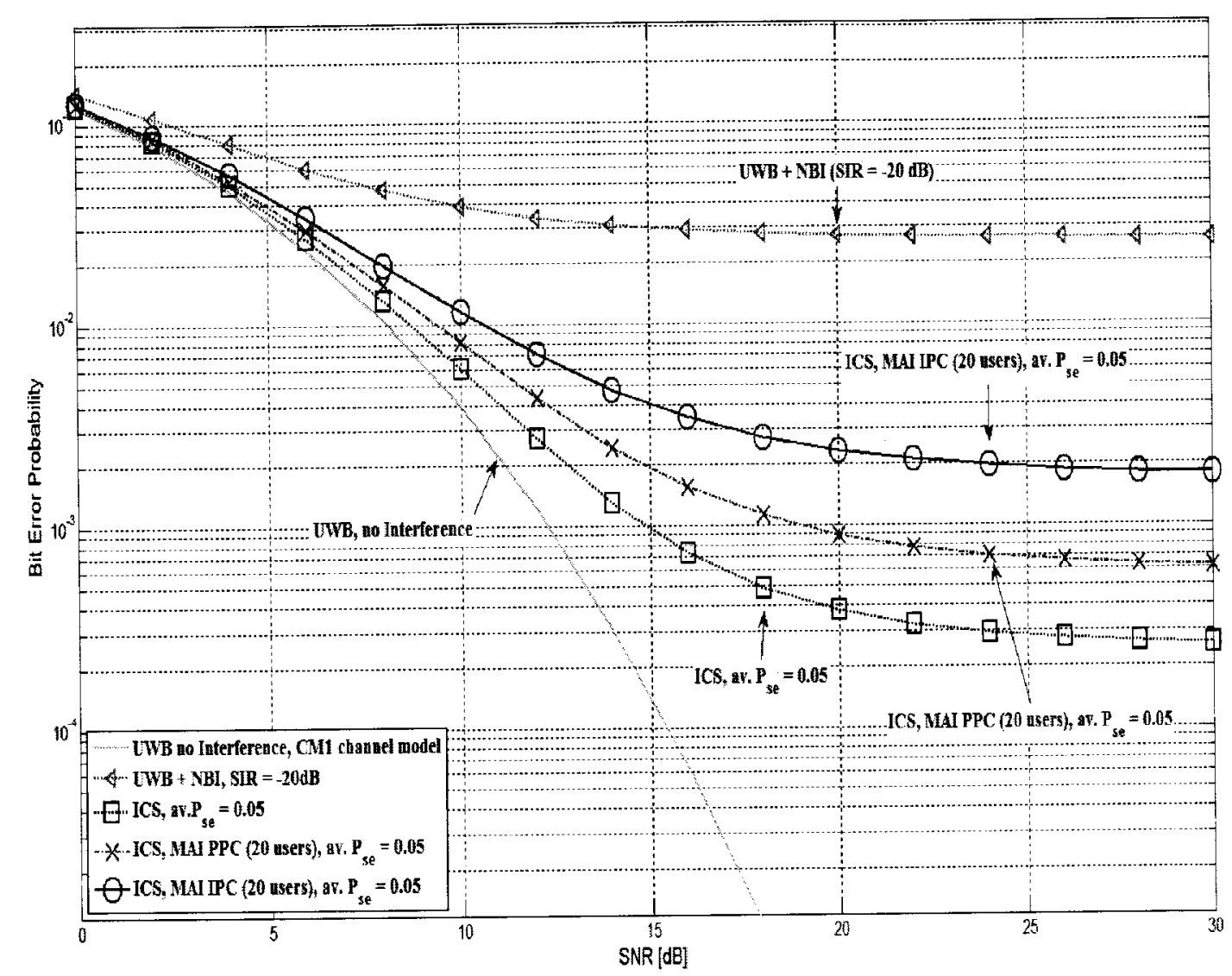

Figure 6.33: ICS performance in the presence of PPC and IPC MAI in the CM1 channel model and SIR $=-20 \mathrm{~dB}$.

filter was simulated as a resonator with quality factor $(Q=35$ and 100$)$. The $3 \mathrm{~dB}$ bandwidth of the notch filter with the previous quality factors will be $165 \mathrm{MHz}$ and $57 \mathrm{MHz}$ respectively for a WLAN NBI signal with center frequency $=5.745 \mathrm{GHz}$.

The comparison is done in two scenarios: 1) the notch filter is assumed perfectly tuned to the center frequency of the NBI signal. 2) The notch filter is de-tuned and there is a shift between the center frequency of the NBI signal and the notch frequency.

Figures 6.35 and 6.36 depict this comparison in the CM1 and the CM4 channel models respectively. It can be seen that in both channel models, the ICS with av. Pse $=0.05$ and 0.1 outperforms the notch filter with $\mathrm{Q}=35$.

Whereas, the notch filter with $Q=100$ outperforms the ICS in both channel models (for av. Pse $=0.1$, the ICS SNR degradation is less than $4 \mathrm{~dB}$ at BER $=1 \times 10^{-4}$ 


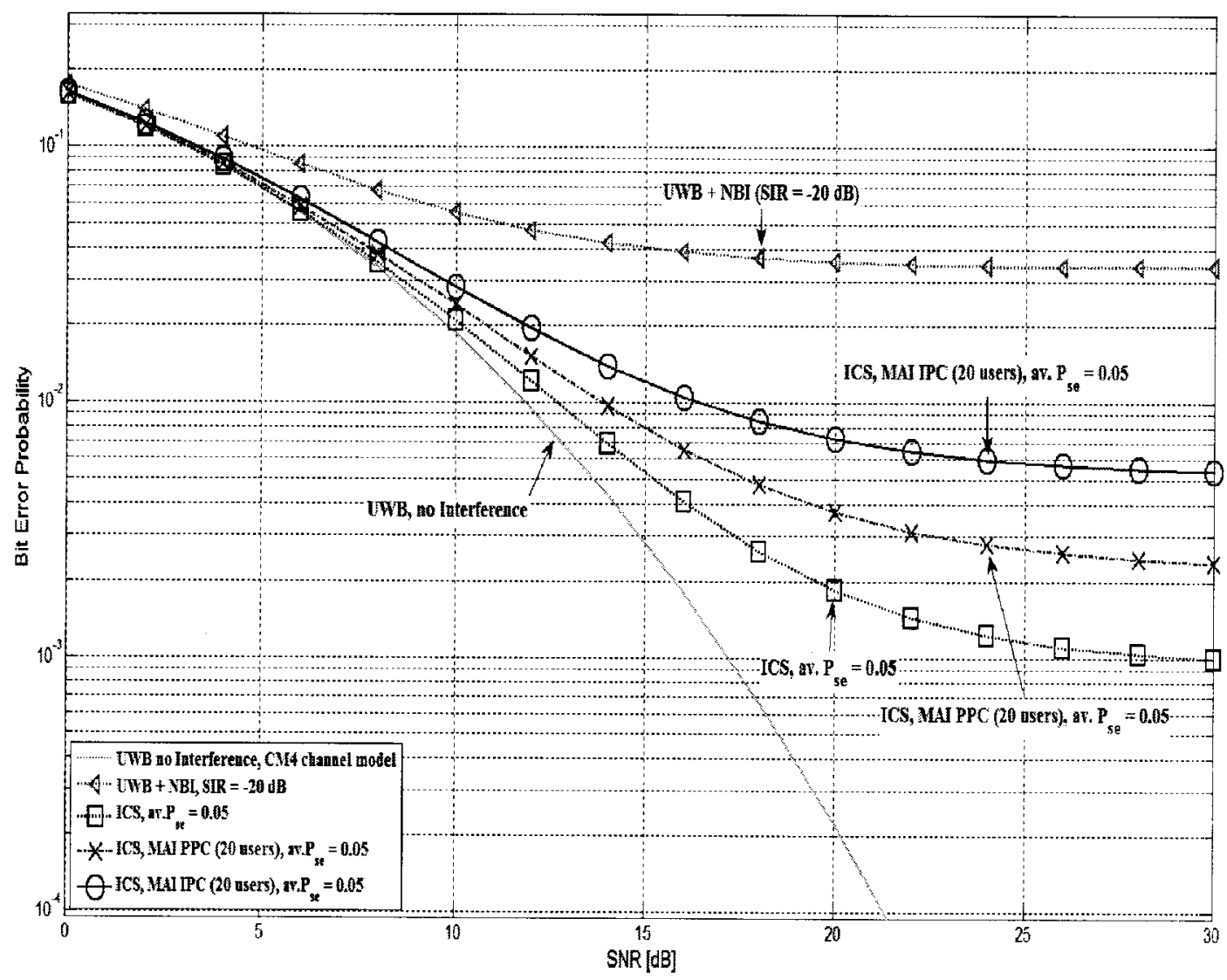

Figure 6.34: ICS performance in the presence of PPC and IPC MAI in the CM4 channel model and SIR $=-20 \mathrm{~dB}$.

and less than $2 \mathrm{~dB}$ at $\mathrm{BER}=1 \times 10^{-3}$ in the $\mathrm{CM} 1$ and the $\mathrm{CM} 4$ channel models respectively).

Finally, figures 6.37 and 6.38 depict this comparison for a de-tuned notch filter in the CM1 and the CM4 channel models respectively. The notch frequency is shifted from the center frequency of the NBI signal by $40 \mathrm{MHz}$ and $60 \mathrm{MHz}$ with a notch filter has a quality factor $Q=100$. It can be seen that the ICS with an av. Pse $=0.1$ outperforms the de-tuned notch filter in both the CM1 and the CM4 channel models. 


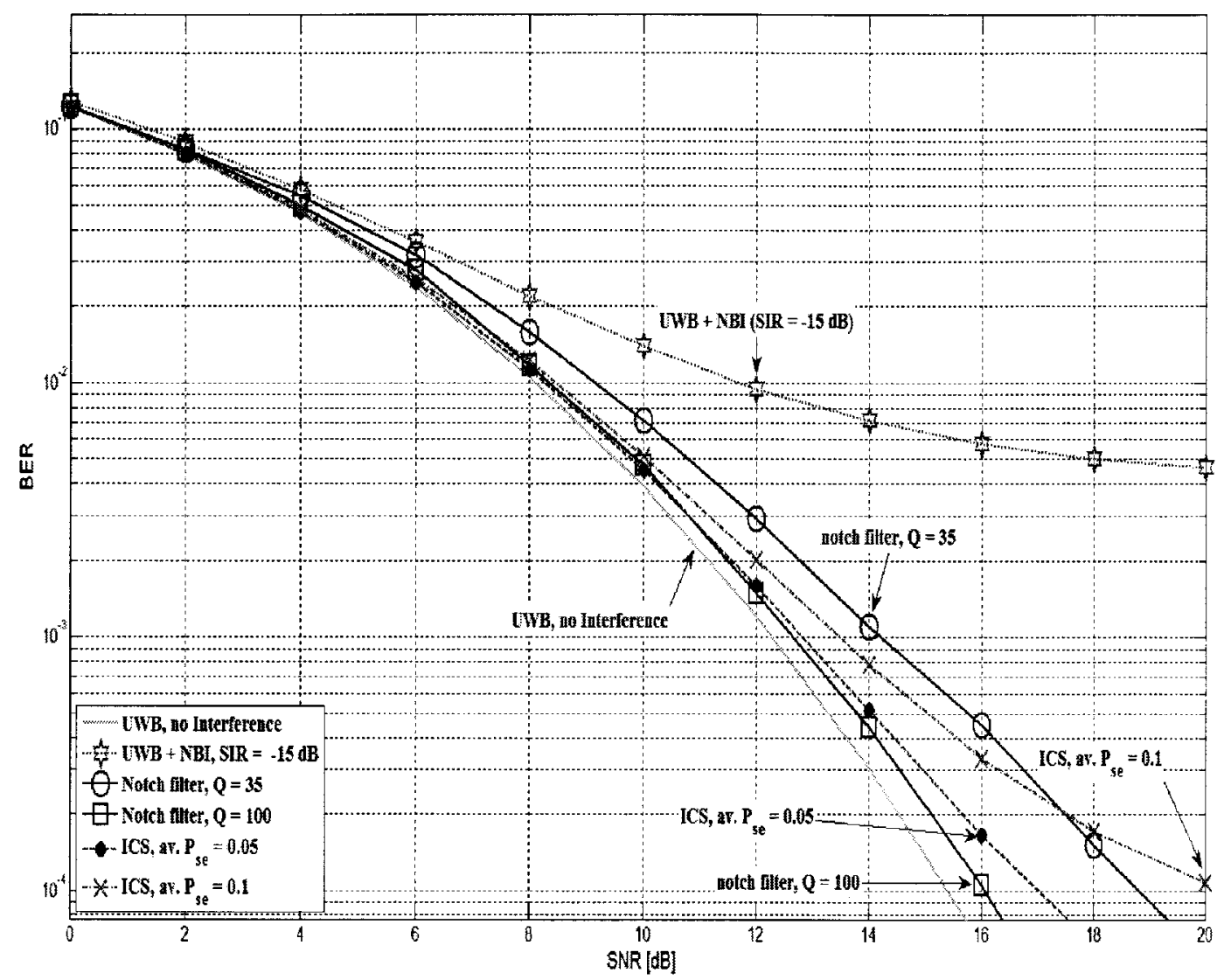

Figure 6.35: Performance comparison with a notch filter in the CM1 channel model and SIR $=-15 \mathrm{~dB}$.

\subsection{IEEE802.11n Interference on IR-UWB Sys- tems}

In our previous work, we had focused on modeling the NBI signal as the OFDM based IEEE802.11a WLAN. However, in the following subsections we will try to investigate the impact of another model of the NBI signal, which is the OFDM based IEEE802.11n WLAN on the performance of UWB system. We will start by giving an overview of the IEEE802.11n standards, followed by depicting its impact on the performance of a TH-PPM UWB system, assuming we are operating in AWGN channel environment. 


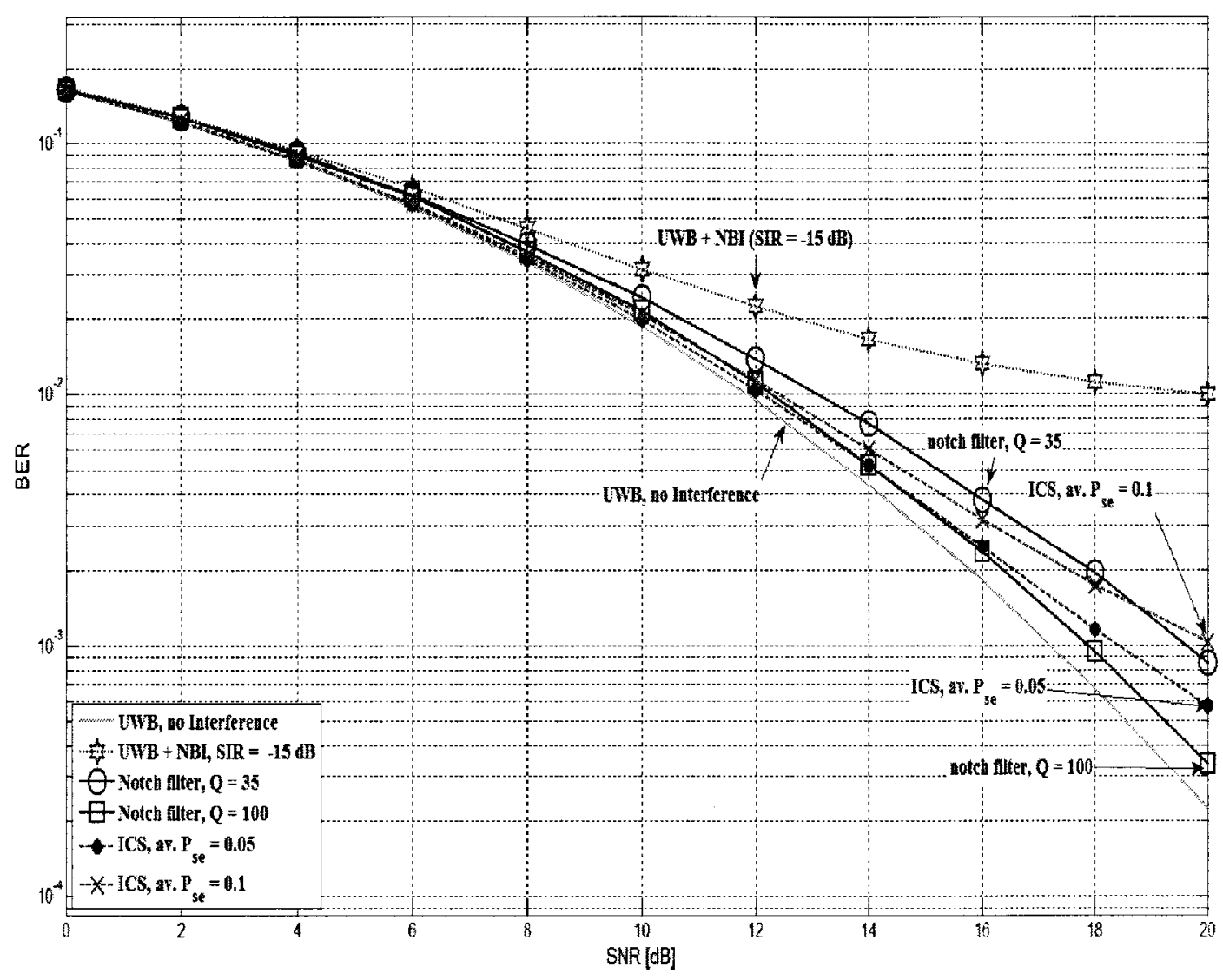

Figure 6.36: Performance comparison with a notch filter in the $\mathrm{CM} 4$ channel model and $\mathrm{SIR}=-15 \mathrm{~dB}$.

\subsubsection{Overview of the IEEE802.11n Standard}

The IEEE802.11n system is an extension of the IEEE802.11 standards for wireless local area networks. The 802.11 task group focused on increasing the peak throughput $^{8}$, which can be achieved either by increasing the peak data rate, improving the medium access control (MAC) layers or both.

The IEEE802.11n amendment describes enhancements to both the physical and MAC layers. Focusing on the modifications to the physical layer, specifically:

1. The use of two $20 \mathrm{MHz}$ bandwidth streams "channel bonding".

2. The use of multiple transmit and receive antennas, which can either be used for

${ }^{8}$ Their initial goal was to achieve $100 \mathrm{Mbps}$ net throughput, after subtracting all the overhead for protocol management features like preambles, inter-frame spacing, and acknowledgments. 


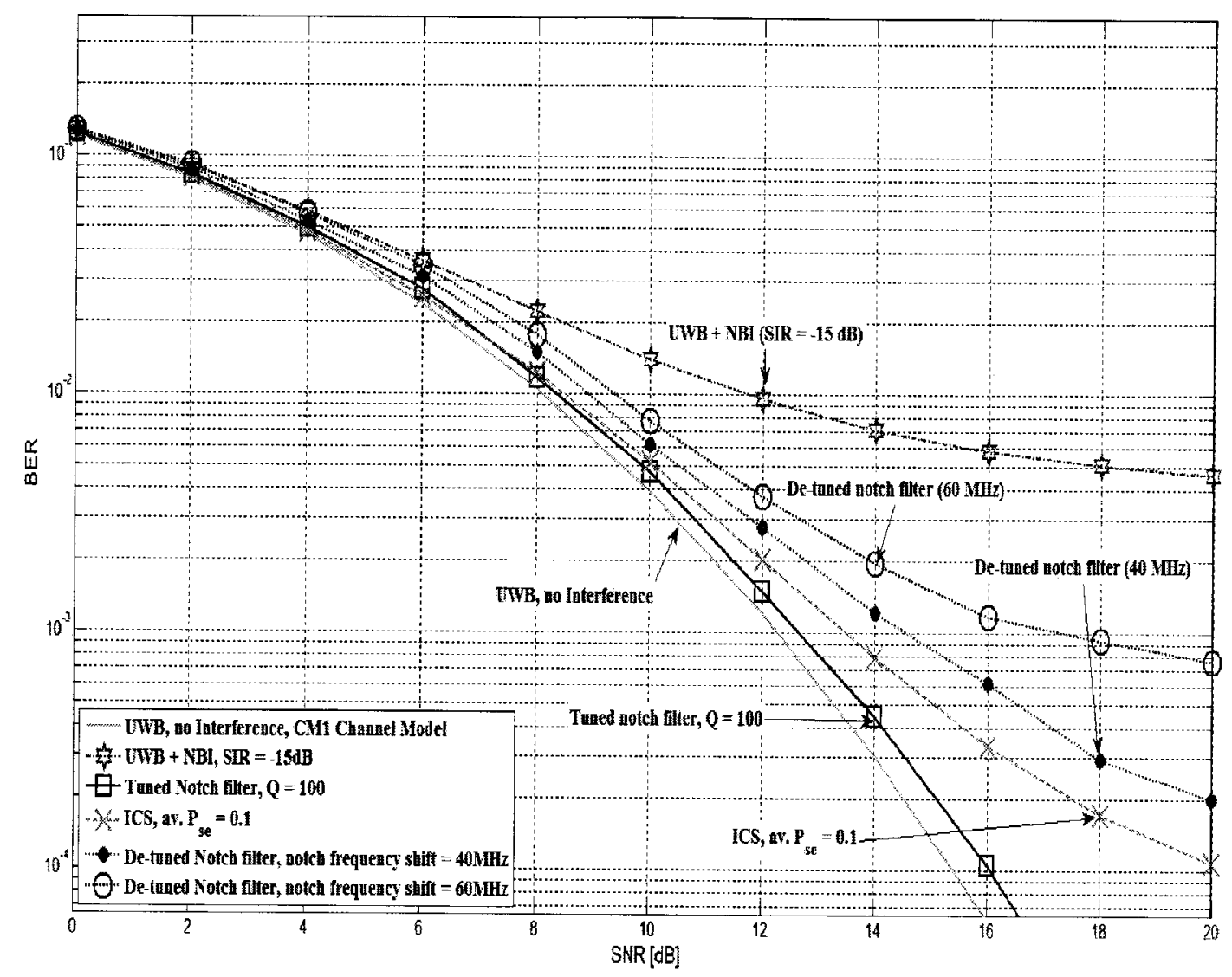

Figure 6.37: Performance comparison with a de-tuned notch filter in the CM1 channel model, $\mathrm{Q}=100$ and $\mathrm{SIR}=-15 \mathrm{~dB}$.

spatial diversity or alternatively as a Multiple-Input Multiple-Output (MIMO) system.

3. The use of improved bit and frame encoding techniques.

4. Beamforming is suggested to extend the effective range and create more robust coverage.

5. Reduces the Guard Interval from 800 nanoseconds to 400 nanoseconds. This small change increases the symbol rate by 10 percent.

6. More available spectrum as the IEEE802.11n supports both $2.4 \mathrm{GHz}$ and $5 \mathrm{GHz}$ bands. 


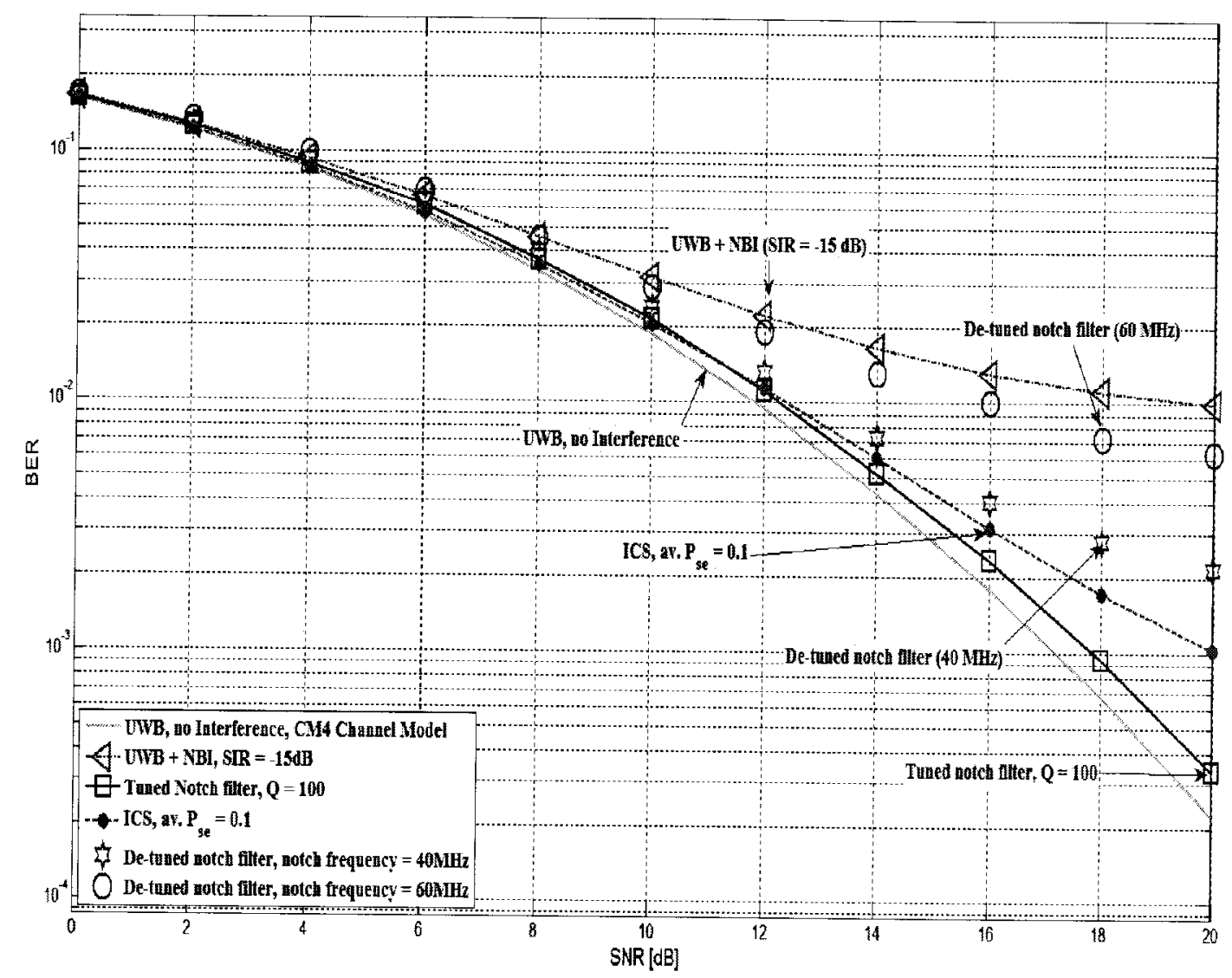

Figure 6.38: Performance comparison with a de-tuned notch filter in the CM4 channel model, $\mathrm{Q}=100$ and $\mathrm{SIR}=-15 \mathrm{~dB}$.

Table 6.1 summarizes the specifications of both IEEE802.11a and IEEE802.11n WLAN systems.

The $40 \mathrm{MHz}$ channel allocations for the IEEE802.11n in the $5 \mathrm{GHz}$ frequency band is depicted in table 6.2 .

It is worth mentioning that, $20 \mathrm{MHz}$ channel allocation also exist for the $802.11 \mathrm{n}$ systems as described in Section 17.3.8.3 in the 802.11 standards [91] as recommended in the draft in Section 20.3.1.4 [3].

Figure 6.39 depicts the data mapping technique that is suggested for the IEEE802.11n WLAN $40 \mathrm{MHz}$ channel, where the channel is divided into 128 sub-carriers. The signal is transmitted on sub-carriers -58 to -2 and 2 to 58 . Sub-carriers " 0 and 1 " in addition to sub-carriers "59-70" are left unmodulated "Null value". 
Table 6.1: Specifications of the IEEE $802.11 \mathrm{a} / \mathrm{n}$ Systems.

\begin{tabular}{|c|c|c|}
\hline Characteristics & IEEE802.11a & IEEE802.11n \\
\hline \hline Standardization & Standard & $\begin{array}{c}\text { Two Proposals } \\
\text { WWiSE \& TGnSync }\end{array}$ \\
\hline Net Throughput & $54 \mathrm{Mbps}$ & goal (greater than $100 \mathrm{Mbps})$ \\
\hline Operating Band & $5 \mathrm{GHz}$ & $2.4 \mathrm{GHz}$ and $5 \mathrm{GHz}$ \\
\hline Channel Bandwidth & $20 \mathrm{MHz}$ & $20 \mathrm{and} /$ or $40 \mathrm{MHz}$ \\
\hline Info. Sub-Carriers & 52 & $56(20 \mathrm{MHz})$ or $108(40 \mathrm{MHz})$ \\
\hline Pilot Tones & 4 & $4(20 \mathrm{MHz})$ or $6(40 \mathrm{MHz})$ \\
\hline Frequency Spacing & $0.3125 \mathrm{MHz}$ & $0.3125 \mathrm{MHz}$ \\
\hline Modulation & OFDM & OFDM \\
\hline Code Rate & $2 / 3 \mathrm{and} 3 / 4$ & $2 / 3,3 / 4$ and $5 / 6$ \\
\hline Guard Interval & $800 \mathrm{~ns}$ & $400 \mathrm{~ns}$ or $800 \mathrm{~ns}$ \\
\hline Antenna & Single & MIMO \\
\hline
\end{tabular}

Table 6.2: Summary of IEEE802.11n $40 \mathrm{MHz}$ Channel Allocation in the $5 \mathrm{GHz}$ Frequency Band [3].

\begin{tabular}{|c|c|c|}
\hline Region & Band $(\mathrm{GHz})$ & Frequencies $(\mathrm{MHz})$ \\
\hline \hline US/Europe & $5.15-5.25$ & $5180,5200,5220,5240$ \\
\hline US/Europe & $5.25-5.35$ & $5260,5280,5300,5320$ \\
\hline US/Europe & $5.50-5.70$ & $5500,5520,5540,5560,5580$ \\
\hline & & $5600,5620,5640,5660,5680$ \\
US & $5.725-5.825$ & $5745,5765,5785,5805$ \\
\hline
\end{tabular}




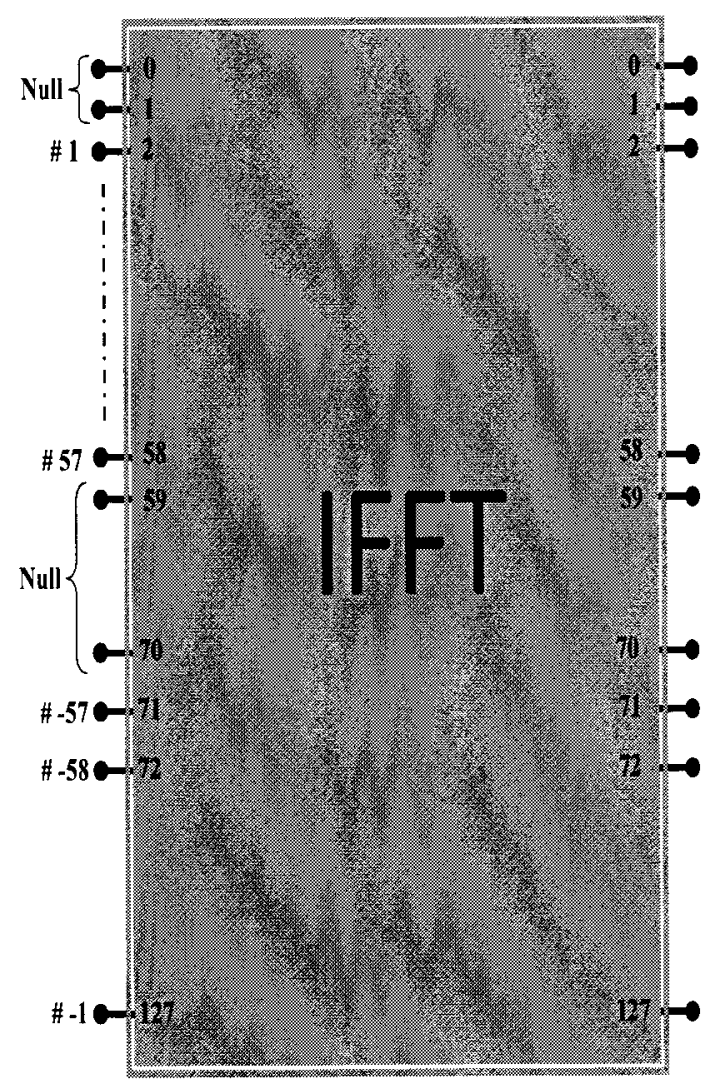

Figure 6.39: Data mapping scheme for the IEEE802.11n WLAN. 


\subsection{IEEE802.11n Interference Analysis}

In this section, the effect of interference on IR-UWB communication systems due to the presence of the IEEE802.11n WLAN system is evaluated.

In order to evaluate such impact in AWGN environment, we followed the same approach as presented in [92]. It has been shown that the assumption of sum of tone interferers is a good approximation for an OFDM interferer.

The BER performance of an IR-UWB system in the presence of NBI signal, modeled as the IEEE $802.11 n^{9}$ can be evaluated as [92]

$$
\begin{aligned}
P_{e}= & Q\left(\sqrt{\frac{E_{b}}{N_{o}} \cdot(1-\varrho)}\right)+1 / \pi \int_{0}^{\infty}\left[1-\prod_{n=1}^{N_{i}} J_{o}\left(\omega \sqrt{\frac{I_{n}}{C} \cdot \frac{\left|M\left(f_{n}\right)\right|^{2}}{T_{b}} \cdot \frac{2}{(1-\varrho)^{2}}}\right)\right] \\
& \frac{\sin (\omega)}{\omega} \cdot \exp \left(-\frac{\omega^{2}}{2} \cdot \frac{N_{o}}{E_{b}} \cdot \frac{1}{(1-\varrho)}\right) d \omega
\end{aligned}
$$

Figure 6.40 depicts a comparison between the performance of a TH-PPM UWB system in the presence of the Wi-Fi802.11a and its performance in the presence of the Wi-Fi802.11n NBI signal, with values: $T_{b}=10 \mathrm{~ns}, \varrho=-0.824, \delta=0.068 \mathrm{~ns}, \tau_{p}$ $=0.192 \mathrm{~ns}$, and $\operatorname{SIR}=0,-5,-10$ and $-15 \mathrm{~dB}$.

It can be seen that, the same impact can be achieved by the presence of either the Wi-Fi802.a or Wi-Fi802.n WLAN on a TH-PPM UWB system. Thus, we can conclude that in the presence of the IEEE802.11n WLAN NBI signal; the canceller scheme can provide the same performance enhancement to IR-UWB communication systems as was provided in the scenario of the presence of the IEEE802.11a WLAN NBI signal.

\footnotetext{
${ }^{9}$ The IEEE $802.11 \mathrm{n}$ is approximated as the sum of $N_{i}$ tone interferers.
} 


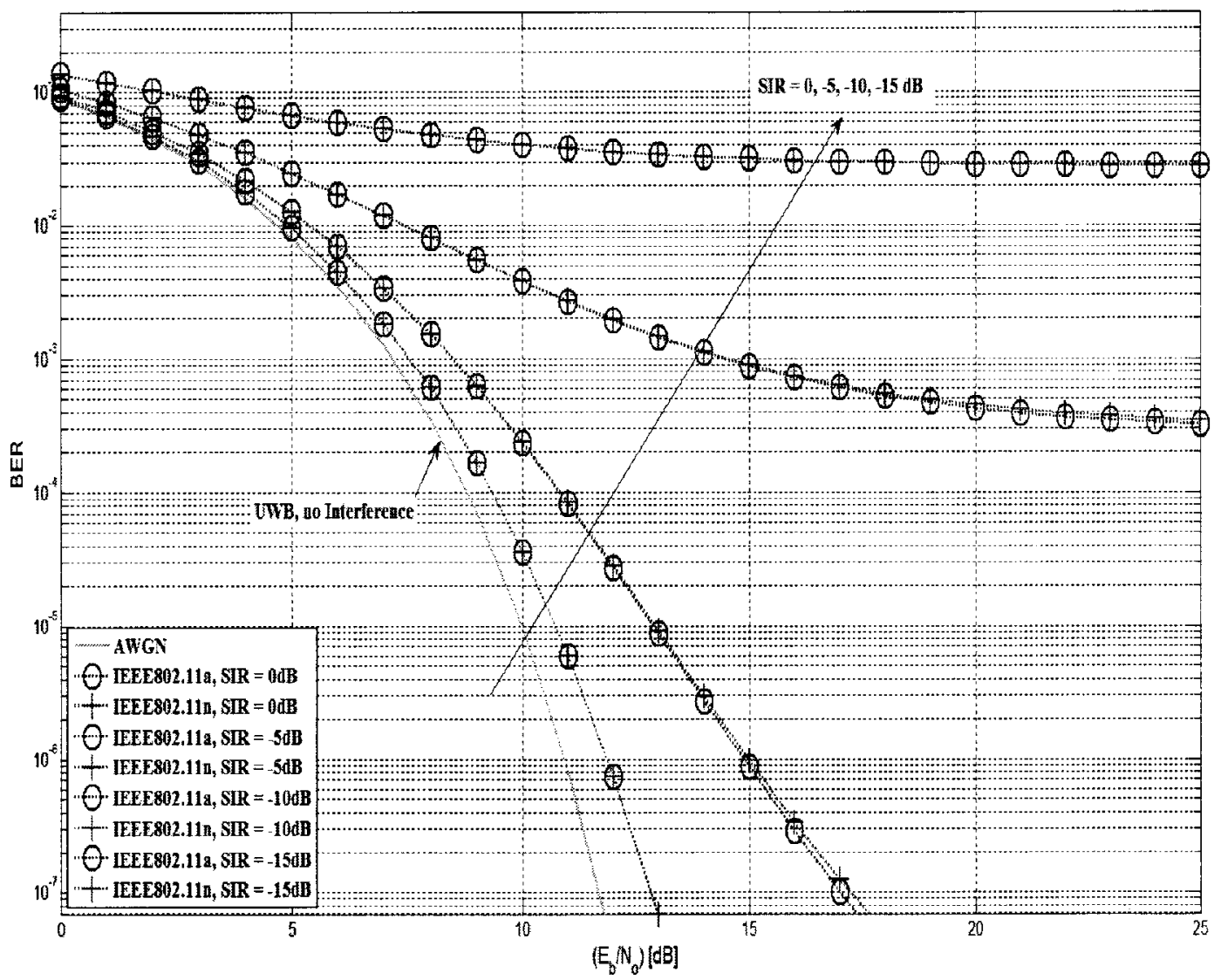

Figure 6.40: IEEE802.11a versus IEEE802.11n impact on a TH-PPM UWB system in AWGN channel. 


\section{Chapter 7}

\section{Conclusions and Suggestions for Future Work}

\subsection{Conclusion and Summary}

The work presented in the thesis investigates the impact of narrowband interference signals on the performance of impulse radio ultra wide-band communication systems. The importance of this investigation arises from the fact that the deployment of UWB systems, which may overlap with several frequency bands allocated to narrowband radio-communication services, requires the coexistence with these narrowband radio systems with the assurance of minimum mutual interference.

However, due to the power restrictions imposed on the UWB communication systems by FCC, the narrowband services that operate in or near the operating band of the UWB systems may exhibit higher PSD levels when compared to the PSD of the UWB systems as seen by a UWB receiver. Thus, one would expect degradation on the BER performance of the UWB system.

To this end, we investigated the impact of NBI signals on the performance of IR-UWB communication systems in various UWB channel models. A tractable analytical BER closed form expression had been developed for a TH-PPM system in the presence of NBI signal modeled as a single tone interferer in a Log-normal frequency selective fading channel.

It had been shown that deterioration in the UWB BER performance is expected due to the presence of NBI signal. Degradation on the performance of the UWB system is also noticeable by increasing the $\mathrm{dB}$-spread value within the range dedicated for UWB communication systems. 
An analytical closed form expression for predicting the BER performance of TH-PPM and DS-BPSK UWB systems in the presence of multiple tone interferers in a Log-normal flat fading channel using a Gaussian Hermite quadrature expansion had been derived. It had been shown also that the DS-BPSK system outperforms the TH-PPM system, as measured by the average BER.

The BER performance of a DS-BPSK system in the presence of the standard WLAN IEEE802.11a signal in the IEEE802.15.3a UWB channel models had been analytically derived. It had been shown that the performance of the DS-UWB system can severely deteriorated due to the presence of the IEEE802.11a interference signal. It had been shown also that an enhancement in the performance of the UWB system is expected by increasing the number of the Rake receiver fingers at the expense of increasing the system complexity.

This thesis also proposes an interference cancellation scheme that is capable of suppressing the impact of such interference and enhancing the performance of IRUWB communication systems. This cancellation scheme attempts to demodulate and to regenerate the standard WLAN OFDM based IEEE802.11a interference signal so it can be subtracted from the incoming received signal at the input of the UWB receiver.

In order to thoroughly analyze the performance of such canceller scheme, the performance of the OFDM NBI receiver has been initially addressed. The impact of UWB signal on the performance of the narrowband receiver had been investigated, where it had been shown that the UWB interference signal could severely deteriorate the performance of the narrowband receiver, especially when the UWB signal power is greater than the narrowband signal power. However, the impact of the UWB interference signal decreases for large narrowband signal power and can be neglected. It had been shown also that the probability of multiple symbol errors for the OFDM narrowband receiver is highly dependent on the number of error symbols in a block of length $\mathrm{N}$ symbols.

The effectiveness of the interference cancellation scheme in mitigating the impact of NBI signal on the performance of IR-UWB systems has been measured in various UWB channel models such as the AWGN environment, Nakagami-m and Log-normal flat fading channels and the IEEE802.15.3a UWB fading channel models. 
The obtained analytical results are validated with the aid of simulation.

The performance of the interference cancellation scheme had been also investigated in different scenarios of operation, such as the impact of the presence of symbol misalignment timing error between the regenerated NBI signal and the incoming received NBI signal at the input of the subtraction process. It had been shown that for a timing misalignment error equals to $1 \%$ of the NBI symbol duration the canceller scheme performance had been degraded.

The capability of the canceller scheme to mitigate the interference generated from the presence of two WLAN NBI signals had been also investigated. It had been shown the capability of the canceller scheme to mitigate such interference with an additional SNR equals to $2 \mathrm{~dB}$ at $\mathrm{BER}=1 \times 10^{-4}$ and $3 \mathrm{~dB}$ at $\mathrm{BER}=1 \times 10^{-3}$ in the CM1 and the CM4 IEEE802.15.3a channel models respectively.

The impact of the presence of MAI on the performance of the proposed canceller scheme had been also investigated assuming the operation in perfect and imperfect power control scenarios for the interfering users. It had been shown that the performance of the canceller scheme could be affected by increasing the number of interfering user.

Finally, in order to measure the effectiveness of such canceller scheme, its performance had been compared with the performance obtained by using a notch filtcr characterized by different values of its quality factor in the IEEE802.15.3a UWB channel models. The comparison is achieved in two cases, perfectly tuned and de-tuned notch filter ${ }^{1}$. It had been shown that for a perfectly tuned notch filter, the canceller scheme outperforms the notch filter with a quality factor $Q$ equals to 35. For $Q$ equals to 100 , the notch filter outperforms the proposed canceller scheme. However, in a de-tuned scenario the canceller scheme outperforms the notch filter.

\footnotetext{
${ }^{1}$ With respect to the center frequency of the NBI signal.
} 


\subsection{Suggestions for Future Research}

1. It has been shown that, the performance analysis for UWB systems has been obtained with a perfect synchronization assumption. As known, UWB systems transmit information using extremely short pulses on hostile channels, for which perfect synchronization is very difficult to achieve. On the other hand, imperfect synchronization of a UWB system will seriously deteriorate its performance. It is of interest to investigate the performance of both UWB systems and such canceller scheme in imperfect synchronization environment.

2. The UWB communication systems are characterized by providing very high data rates. In high data rate systems, it may be impossible to prevent leakage of multi-paths among symbols in both coherent and non-coherent UWB receivers. Many literatures have investigated the impact of inter symbol interference on the performance of coherent and non-coherent UWB receivers. However, the impact of inter symbol interference on the error rate performance of the canceller scheme has not been addressed and it is worth to be investigated.

3. Through our research, the impact of multiple access interference on the performance of the canceller scheme has been investigated, where the multiple access interference was assumed to have a Gaussian distribution. However, this assumption cannot give accurate results even when a large number of interferers are present. It is of interest to investigate a more appropriate statistical model for the multiple access interference, showing its impact on the performance of such canceller scheme.

4. Although the single-band impulse radio is the original proposal for UWB communications, multi-band UWB systems have also attracted much interest because of their advantages. Particularly, multi-carrier, multi-band systems use OFDM techniques to transmit the information on each of the sub-bands gain more attention because of nice properties of OFDM. It is of interest to investigate the effectiveness of the canceller scheme to mitigate the impact of NBI on the performance of the multi-band UWB systems.

5. The operation of the proposed interference canceller scheme was achieved assuming perfect channel estimation for both the desired UWB and the NBI 
signals. It is of interest to investigate the effect of imperfect channel estimation for both signals on the performance of such canceller scheme.

6. It has been shown the capability of the canceller scheme to suppress the impact of NBI signals, assuming that these NBI signals have an OFDM based structure such as the standard IEEE802.11a WLAN signals. Since the idea of the canceller scheme is based on demodulating and regenerating the NBI signal. Several problems in OFDM based signals are worthy of investigation. OFDM resistance to frequency selective fading comes at the price of greater inter-carrier interference from its own sub-band transmissions and greater sensitivity to dynamic range. All these problems and their impact on the canceller performance need to be carefully investigated.

7. It has been shown that the cancellation strategy of the NBI signal is based on a full tone cancellation. However, due to the nature of the NBI channel environment, channel fade could differ from tone to tone leading to the fact that some tones could be affected by deep fade more than others. It is of interest to adopt a more convenient cancellation technique, and thus improve the canceller BER performance. 


\section{List of References}

[1] T. Wang and Y. Wang, "Capacity of M-ary PAM impulse radio with various derivatives of Gaussian pulse subject to FCC spectral masks," Ninth International Symposium on Computers and Communications, ISCC 2004, vol. 2, pp. $696-701,2004$.

[2] K. Siwiak and D. McKeown, Ultra Wideband Radio Technology. John wiley \& Sons, 2004.

[3] "IEEE P802.11n/D3.00: Draft Part 11: Wireless LAN Medium Access Control (MAC) and Physical Layer (PHY) specifications: Amendment 4: Enhancements for Higher Throughput," September 2007.

[4] Z. Sahinoglu, S. Gezici, and I. Guvenc, Ultra Wideband Positioning Systems: Theoretical Limits, Ranging Algorithms and Protocols. New York: Cambridge University Press, 2008.

[5] H. Arslan, Z. Chen, and M. Benedetto, Ultra Wideband Wireless Communication. New York: John Wiley \& Sons, 2006.

[6] R. Roberts, "XtremeSpectrum CFP document." IEEE P802.15-03/153r8, July 2003.

[7] W. P. Siriwongpairat and K. R. Liu, Ultra Wideband Communications Systems : Multiband OFDM Approach. Wiley-IEEE Press, November 2007.

[8] K. Ryu and A. Kishk, "UWB antenna with single or dual band-notches for lower WLAN band and upper WLAN band," IEEE Transactions on Antennas and Propagation, vol. 57, pp. 3942-3950, December 2009.

[9] M. Benedetto and G. Giancola, Understanding Ultra WideBand Radio Fundamentals. New Jersey, U.S.A: Prentice Hall, 2004.

[10] H. Harada and R. Prasad, Simulation and Software Radio for Mobile Communications. Boston, London: Artech House, 2002.

[11] W. C. Jakes, Microwave Mobile Communications. John Wiley \& Sons, 1974.

[12] M. Hamalainen, R. Tesi, and J. Iinatti, "UWB coexistence with IEEE802.11a and UMTS in modified saleh-valenzuela channel," International Workshop on Ultra Wideband Systems joint with Conference on Ultra wideband Systems and Technologies, pp. 45-49, 18-21 May 2004. 
[13] H. Shao and N. Beaulieu, "An analytical method for calculating the bit error rate performance of Rake reception in UWB multi-path fading channels," IEEE International Conference on Communications, ICC'08, pp. 4855-4860, 19-23 May 2008.

[14] Q. Li-na, G. Zong-liang, and Z. Hong-bo, "ISI cancellation for timing hopping ultra wideband systems," Second International Conference on Communications and Networking in China, CHINACOM '07, pp. 145-148, 2007.

[15] J. Taylor, Ultra wideband Radar Overview. CRC Press, 1995.

[16] M. Win and R. Scholtz, "Ultra wide bandwidth time-hopping spread-spectrum impulse radio for wireless multiple-access communications," IEEE Transactions on Communications, vol. 48, pp. 679-691, 2000.

[17] H. Kikuchi, UWB arrives in Japan. Nikkei Electronics, February 2003. 95-122.

[18] I. Oppermann, M. Hamalainen, and J. Linatti, UWB Theory and Applications. Chichester: John Wiley and Sons, 2004.

[19] J. Conroy, J. Locicero, and D. Ucci, "Communication techniques using monopulse waveforms," Proceedings of IEEE Military Communications Conference, MILCOM'99, vol. 2, pp. 1181-1185, October-November 1999.

[20] M. G. D. Benedetto, UWB Communication Systems: A Comprehensive Overview. Hindawi Publishing Corporation, 2006.

[21] M. Z. Win and R. A. Scholtz, "Impulse radio: How it works," IEEE Communication Letters, vol. 2, no. 2, pp. 51-53, 1998.

[22] F. Ramirez-Mireles, "Signal design for ultra wideband communications in dense multi-path," IEEE Transactions on Vehicular Technology, vol. 51, no. 6, pp. 1517-1521, November 2002.

[23] X. Chen and S. Kiaei, "Monocycles shapes for ultra wideband system," Proceedings IEEE Conference on Ultra Wide band Systems and Technologies, pp. 597 600, 20-23 May 2002, Baltimore, USA.

[24] M. Ghavami, L. Michael, and R. Kohno, Ultra Wideband Signals and Systems in Communication Engineering. John Wiley \& Sons, 2004.

[25] C. Chui and R. Scholtz, "Optimizing tracking loop for UWB monocycles," in IEEE Global Communications Conference, GLOBECOM2003, pp. 425-430, 2003.

[26] B. $\mathrm{Hu}$ and N.C.Beaulieu, "Effects of IEEE802.11a narrowband interference on a UWB communication system," IEEE International Conference on Communications, vol. 4, pp. 2818-2824, May 2005.

[27] M. Chiani and A. Giorgetti, "Coexistence between UWB and narrow-band wireless communication systems," Proceedings of the IEEE, vol. 97, pp. 231-254, February 2009. 
[28] A. F. Molisch, "Ultra wide-band propagation channels," Proceedings of the IEEE, vol. 97, February 2009.

[29] D. Cassioli, M. Win, and A. Molisch, "The ultra wide bandwidth indoor channel: From statistical model to simulations," IEEE Journal on Selected Areas in Communications, vol. 20, no. 6, pp. 1247-1257, August 2002.

[30] J. Kunisch and J. Pamp, "Measurement results and modeling aspects for the UWB radio channel," Proceedings IEEE UWBST, pp. 19-23, 2002.

[31] U. G. Schuster and H. Boelcskei, "Ultra wideband channel modeling on the basis of information-theoretic criteria," IEEE Transactions on Wireless Communications, vol. 6, pp. 2464-2475, 2007.

[32] J. Foerster and Q. Li, "UWB channel modelling contribution from Intel," IEEE Journal on Selected Areas in Communications, vol. 5, no. 2, pp. 128-137, February 1987.

[33] A. Saleh and R. Valenzuela, "A statistical model for indoor multi-path propagation," IEEE Journal on Selected Areas in Communications, vol. 5, no. 2, pp. 128-137, 1987.

[34] W. Lee, "Overview of cellular CDMA," IEEE Transactions on Vehicular Technology, vol. 40, pp. 291-302, May 1991.

[35] S. Niranjayan, A. Nallanathan, and B. Kannan, "Modeling of multiple access interference and BER derivation for TH and DS UWB multiple access systems," IEEE Transactions on Wireless Communications, vol. 5, pp. 2794-2804, 2006.

[36] I. Hosseini and N. Beaulieu, "Bit error rate of TH-BPSK UWB receivers in multiuser interference," IEEE Transactions on Wireless Communications, vol. 8 , pp. 4916-4921, 2009.

[37] N. Beaulieu, H. Shao, and J. Fiorina, "P-order metric UWB receiver structures with superior performance," IEEE Transactions on Communications, vol. 56, no. 10, pp. 1666-1676, October 2008.

[38] S. Niranjayan and N. Beaulieu, "A myriad filter detector for UWB multiuser communication," Proceedings IEEE International Conference on Communications, ICC'08, pp. 3918-3922, May 2008.

[39] J. Proakis, Digital Communications. New York, NY, USA: McGraw-Hill, 1995.

[40] D. Cassioli, M. Win, F. Vatalaro, and A. Molisch, "Performance of lowcomplexity Rake reception in a realistic UWB channel," Proceedings ICC 2002, pp. 763-767, May 2002.

[41] A. Rajeswaran, V. Somayazulu, and J. Foerster, "Rake performance for a pulse based UWB system in a realistic UWB indoor channel," Proceedings ICC 2003 , pp. 2879-2883, May 2003. 
[42] B. Hu and N. Beaulieu, "Comparison of modulation schemes and Rake receiver structures for UWB systems on an IEEE 802.15.3 indoor channel," IEEE Global Telecommunications Conference, GLOBECOM'05, vol. 6, pp. 3493-3497, 2005.

[43] J. Bellorado, S. Ghassemzadeh, L. Greenstein, T. Sveinsson, and V. Tarokh, "Coexistence of ultra wideband systems with IEEE802.11a wireless LANs," IEEE Global Telecommunications Conference, GLOBECOM '03, vol. 1, pp. 410-414, 1-5 December 2003.

[44] J. Foerster, "Ultra wideband technology enabling low-power, high-rate connectivity (invited paper)," Proceedings IEEE Workshop Wireless Communications Networking, September 2002.

[45] L. Zhao and A. Haimovich, "Performance of ultra wideband communications in the presence of interference," IEEE Journal on Selected Areas in Communications, vol. 20, pp. 1684-1691, December 2002, Singapore.

[46] M. Hmlinen and V. Hovinen, "On the UWB system coexistence with GSM900, UMTS/WCDMA, and GPS," IEEE Journal on Selected Areas in Communications, vol. 20, no. 9, pp. 1712-1721, 2002.

[47] M. Hmlinen, J. Iinatti, and R. Tesi, "On the UWB system performance studies in AWGN channel with interference in UMTS band," IEEE Conference on Ultra Wideband Systems and Technologie, pp. 321-325, 2002.

[48] A. Swami, B. Sadler, and J. Turner, "On the coexistence of ultra wideband and narrowband radio systems," IEEE Military Communications Conference, MILCOM'2001, vol. 1, pp. 16-19, 2001.

[49] B. Firoozbakhsh, T. Pratt, and N. Jayant, "Analysis of IEEE802.11a interference on UWB systems," IEEE Conference on Ultra Wideband Systems and Technologies, vol. 1, pp. 16-20, November 2003.

[50] X. Chu and R. Murch, "The effect of NBI on UWB time-hopping systems," IEEE Transactions on Wireless Communications, vol. 3, no. 5, pp. 1431-1436, September 2004.

[51] A. Taha and K. Chugg, "A theoretical study on the effects of interference on UWB multiple access impulse radio," Proceedings IEEE Asilomar Conference on Signals, Systems, Computers, vol. 1, no. 5, pp. 728-732, November 2002, Pacific Grove, CA.

[52] Y. Wang, X. Dong, and I. Fair, "Spectrum shaping and NBI suppression in UWB communications," IEEE Transactions on Wireless Communications, vol. 6, pp. 1944-1952, May 2007.

[53] Z. Honggang, X. Zhou, K. Yazdandoost, and I. Chlamtac, "Multiple signal waveforms adaptation in cognitive ultra wideband radio evolution," IEEE Journal on Selected Areas in Communications, vol. 24, pp. 878-884, April 2006. 
[54] R. Dilmaghani, M. Ghavami, B. Allen, and H. Aghvami, "Novel UWB pulse shaping using prolate spheroidal wave functions," IEEE Proceedings on Personal, Indoor and Mobile Radio Communications, PIMRC2003, vol. 1, pp. 602-606, September 2003.

[55] K. Ohno and T. Ikegami, "Interference detection and mitigation by using multicarrier template wave for pulse based UWB," IEEE Ninth International Symposium on Spread Spectrum Techniques and Applications, pp. 183-187, 28-31 August 2006.

[56] K. Ohno and T. Ikegami, "Interference mitigation study for the coexistence of biphase UWB and multi-band OFDM," IEEE International Conference on Ultra Wideband, ICU2005, pp. 707-712, 5-8 September 2005.

[57] S. Hong, K. Chung, and J. Choi, "Design of a band-notched wideband antenna for UWB applications," Antennas and Propagation Society IEEE International Symposium, pp. 1709-1712, 9-14 July 2006.

[58] Y. D. Dong, W. Hong, Z. Q. Kuai, and J. Y. Zhou, "Band-notched ultra wideband antenna designed and optimized by current distribution analysis," International Conference on Microwave and Millimeter Wave Technology, ICMMT 2008, vol. 3, pp. 1062-1065, 21-24 April 2008.

[59] J. Wang and W. Tung, "Narrowband interference suppression in time-hopping impulse radio ultra wideband communications," IEEE Transactions on Communications, vol. 54, pp. 1057-1067, June 2006.

[60] T. Fischer, B. Kelleci, K. Shi, A. Karsilayan, and E. Serpedin, "An analog approach to suppressing in-band narrow-band interference in UWB receivers," IEEE Transactions on Circuits and Systems I, vol. 54, pp. 941-950, 2007.

[61] M. Lakshmanan and H. Nikookar, "Mitigation of interference from wideband IEEE 802.11a source on UWB wireless communication using frequency selective wavelet packets," International Waveform Diversity and Design Conference, pp. 50-54, 4-8 June 2007.

[62] M. Medley, G. Saulnier, and P. Das, "Radiometric detection of direct-sequence spread spectrum signals with interference excision using the wavelet transform," IEEE International Conference on Communication, ICC'94, vol. 3, pp. 16481652, May 1994.

[63] J. Patti, S. Roberts, and M. Amin, "Adaptive and block excisions in spread spectrum communication systems using the wavelet transform," Asilomar Conference on Signals, Systems, Computers, vol. 1, pp. 293-297, November 1994.

[64] L. Milstein, "Interference rejection techniques in spread spectrum communications," in Proceedings of the IEEE, vol. 76, pp. 657-671, June 1988.

[65] X. Wang and H. V. Poor, Wireless Communication Systems: Advanced Techniques for Signal Reception. Englewood Cliffs, NJ: Prentice Hall, 1st edn ed., 2004 . 
[66] H. Quach and A. Dinh, "Narrowband interference elimination in UWB communications systems," Canadian Conference on Electrical and Computer Engineering, CCECE 2007, pp. 1341-1344, 22-26 April 2007.

[67] B. Gaffney and A. D. Fagan, "Adaptive nonlinear narrow band interference rejection in ternary DS-UWB," IEEE International Conference on Communications, vol. 7, pp. 3146-3150, June 2006.

[68] S. Verdu, Multiuser Detection. Cambridge: Cambridge University Press, 1st edn ed., 1998.

[69] J. Foerster, "Interference modeling of pulse-based UWB waveforms on narrowband systems," IEEE 55th Vehicular Technology Conference, VTC 2002, vol. 4, pp. 1931-1935, Spring 2002.

[70] R. E. Ziemer and R. L. Peterson, Introduction to Digital Communication,. Prentice Hall, 2001.

[71] A. Giorgetti, M. Chiani, and M. Z. Win, "The effect of narrowband interference on wideband wireless communication systems," IEEE Transactions on Communications, vol. 53, no. 12, December 2005.

[72] I. S. Gradshteyn and I. M. Ryzhik, Tables of Integrals, Series, and Products. San Diego: CA: Academic, 4 ed., 1980.

[73] H. Hashemi, "The indoor radio propagation channel," Proceedings of the IEEE, vol. 81, pp. 943-968, July 1993a.

[74] Ehab M. Shaheen and M. El-Tanany, "BER analysis of UWB systems in the presence of narrowband interference in log-normal multi-path fading channels," IEEE Military International Conference, Milcom2009, pp. 1-7, 18-21 October 2009.

[75] J. M. Holtzman, "On using perturbation analysis to do sensitivity analysis: Derivatives vs. differences," 28th IEEE Conference on Decision Control, vol. 3, pp. 2018-2023, December 1989.

[76] N. Beaulieu, A. Abu-Dayya, and P. Mclane, "Estimating the distribution of a sum of independent log-normal random variables," IEEE Transactions on Communications, vol. 43, pp. 2869-2873, December 1995.

[77] L. F. Fenton, "The sum of log-normal probability distributions in scatter transmission systems," IRE Transactions on Communications Systems, vol. 8, pp. 57 67, March 1960.

[78] R. Barakat, "Sums of independent log-normally distributed random variables," Journal of the Optical Society of America, vol. 66, pp. 211-216, 1976.

[79] N. C. Beaulieu and Q. Xie, "An optimal log-normal approximation to lognormal sum distributions," IEEE Transactions on Vehicular Technology, vol. 53, pp. 479-489, 2004. 
[80] F.Heliot, M.Ghavami, and M.R.Nakhai, "An accurate closed-form approximation of the average probability of error over a log-normal fading channel," IEEE Transactions on Wireless Communications, vol. 7, pp. 1495-1509, May 2008.

[81] S. L. Cotton and W. G. Scanlon, "Higher order statistics for log-normal smallscale fading in mobile radio channels," IEEE Antennas and Wireless Propagation Letters, vol. 6, pp. 540-543, 2007.

[82] J. C. Filho and M. D. Yacoub, "Nakagami-m approximation to the sum of M non-identical independent nakagami-m variates," Electronics Letters, vol. 40, pp. 951-952, 22 July 2004.

[83] Ehab M. Shaheen and M. El-Tanany, "Narrowband interference impact on the performance of UWB communication systems in log-normal flat fading channels," IEEE 71st Vehicular Technology Conference, VTC2010, 16-19 May 2010.

[84] M. Abramowitz and I. Stegun, Handbook of mathematical functions with formulas, graph, and mathematical tables. Dover, 9ed., 1971.

[85] Ehab M. Shaheen and M. El-Tanany, "The impact of narrowband interference on the performance of UWB systems in the IEEE802.15.3a channel models," IEEE 23rd Annual Canadian Conference on Electrical and Computer Engineering, CCECE2010, 2-5 May 2010.

[86] C. Chong and S. Yong, "A generic statistical-based UWB channel model for highrise apartments," IEEE Transactions on Antennas Propagation, pp. 2389-2399, 2005.

[87] J. Foerster and et al., "Channel modelling sub-committee report final," tech. rep., P802.15-02/490r1-SG3a, February 2003.

[88] Y. Chen and N. Beaulieu, "Moment based interference analysis of CM1, CM2, CM3 and CM4 UWB systems," IEEE Global Telecommunications Conference, GLOBECOM'O7., pp. 3858-3862, 26-30 November 2007.

[89] J. Zhang, R. Kennedy, and T. Abhayapala, "Performance of Rake reception for ultra wideband signals in a log-normal-fading channel," Proceedings IWUWBS, pp. 5-9, 2003.

[90] G. Giancola, L. D. Nardis, and M. G. D. Benedetto, "Multi user interference in power-unbalanced ultra wide band systems: Analysis and verification," IEEE Conference on Ultra Wideband Systems and Technologies, pp. 325-329, 2003.

[91] "IEEE Std. 802.11-2007, IEEE Standard for Information technology Telecommunications and information exchange between systems Local and metropolitan area networks Specific requirements, Part 11: Wireless LAN Medium Access Control (MAC) and Physical Layer (PHY) Specs," September 2007.

[92] A. Giorgetti and D. Dardari, "The impact of OFDM interference on THPPM/BPAM transmission systems," IEEE 61st Vehicular Technology Conference, vol. 2, pp. 1037-1042, June 2005. 